\title{
Identification of Key Genes and Signaling Pathways Associated with the Progression of Glioblastoma multiform
}

Basavaraj Vastrad $^{2}$, Chanabasayya Vastrad ${ }^{4 *}$, Iranna Kotturshetti ${ }^{5}$

1. Department of Biochemistry, Basaveshwar College of Pharmacy, Gadag, Karnataka 582103, India.

2. Biostatistics and Bioinformatics, Chanabasava Nilaya, Bharthinagar, Dharwad 580001 , Karanataka, India.

3. Department of Ayurveda, Rajiv Gandhi Education Society`s Ayurvedic Medical College, Ron 562209, Karanataka, India.

* Chanabasayya Vastrad

channu.vastrad@gmail.com

Ph: +919480073398

Chanabasava Nilaya, Bharthinagar,

Dharwad 580001 , Karanataka, India 


\begin{abstract}
Genomic features have been gradually regarded as part of the basics to the clinical diagnosis, prognosis and treatment for glioblastoma multform (GBM). However, the molecular modifications taking place during the advancement of GBM remain unclear. Therefore, recognition of potential important genes and pathways in the gastric cancer progression is important to clinical practices. In the present study, gene expression dataset (GSE116520) of GBM were selected from the Gene Expression Omnibus (GEO) database and were further used to identify differentially expressed genes (DEGs). Then, pathway and Gene Ontology (GO) enrichment analyses were conducted, and a protein-protein interaction (PPI) network was constructed to explore the potential mechanism of GBM carcinogenesis. Significant modules were discovered using the PEWCC1 plugin for Cytoscape. In addition, a target gene - miRNA regulatory network and target gene - TF regulatory network in GBM were constructed using common deregulated miRNAs, TFs and DEGs. Finally, we carried on validation of hub genes by UALCAN, cBioporta, human protein atlas, ROC (Receiver operating characteristic) curve analysis, RT-PCR and immune infiltration analysis. The results indicated that a total of 947 differential expressed genes (DEGs) (477 up regulated and 470 down regulated) was identified in microarray profiles. Pathway enrichment analysis revealed that DEGs (up and down regulated ) were mainly associated in reactive oxygen species degradation, ribosome, homocarnosine biosynthesis and GABAergic synapse, whereas GO enrichment analyses revealed that DEGs (up and down regulated) were mainly associated in macromolecule catabolic process, cytosolic part, synaptic signaling and synapse part as the main pathways associated in these processes. Finally, we filtered out hub genes, including MYC, ARRB1, RPL7A, SNAP25, SOD2, SVOP, ABCC3 and ABCA2,
\end{abstract}


medRxiv preprint doi: https://doi.org/10.1101/2020.12.21.20248616; this version posted December 23, 2020. The copyright holder for this

from the all networks. Validation of hub genes suggested the robustness of the above results. In conclusion, these results provided novel and reliable biomarkers for GBM, which will be useful for further clinical applications in GBM diagnosis, prognosis and targeted therapy.

Key words: glioblastoma multiform; differentially expressed genes; pathways; prognostic; hub genes

\section{Introduction}

Glioblastoma multform (GBM) is one of the most malignant glial tumors with the 5 -year survival rate $9.8 \%$ [1]. In current years, although novel advances have been made in multimodal treatment of cancers, indigent prognosis and high mortality of GBM has remained consistent. About 296,851 individuals in the global were diagnosed with GBM in 2018, of which 241,037 people died, resulting in roughly equal morbidity and mortality [2]. Present situation, radiotherapy [3], chemotherapy [4] and surgical resection [5] are still the most effective way of improving the survival rate of GBM patients. However, GBM is difficult to diagnose in the early stages due to its concealed location and uncommon clinical symptoms. In most cases, majority of the patients tend to be in the final stage when they are clinically diagnosed and lose the chance of radiotherapy, chemotherapy and surgical resection. Therefore, the genes associated in the occurrence and advancement of GBM needs to be explored, which will contribute to the finding of diagnostics markers, prognostic markers and therapeutic targets of GBM.

The underlying molecular pathogenesis of GBM remains inadequately unexplored. Therefore, it is encourage the need to advance a further diagnose the etiological factors, molecular mechanisms, and pathways of GBM to discover novel diagnostic and treatment strategies for GBM. Fortunately, with the development of highthroughput DNA microarray analyses, various genes and pathways have been demonstrated to be correlated with the genesis and progression of GBM [6]. Genes such as NDRG2 [7], PARK2 [8], WT1 [9], RB1 [10] and HDAC (histone deacetylase) [11] were linked with pathogenesis of GBM. Pathways such as Akt pathway [12], EGFR-MEK-ERK signaling pathway 
medRxiv preprint doi: https://doi.org/10.1101/2020.12.21.20248616; this version posted December 23, 2020. The copyright holder for this

[13], AMPK-TSC-mTOR signaling pathway [14], NFkB pathway [15] and MAP kinase pathway [16] were involved in progression of GBM. Therefore, finding differentially expressed genes (DEGs) and pathways, illuminate the interactions network among them, are important for GBM.

In this study, we downloaded the original data (GSE116520) from Gene Expression Omnibus (GEO, http://www.ncbi.nlm.nih.gov/geo/). The differentially expressed genes (DEGs) of normal control from GBM were screened using limma $\mathrm{R}$ bioconductor tool. Subsequently, the pathway and gene ontology (GO) enrichment analysis for DEGs were analyzed. Additionally, we established proteinprotein interaction (PPI) network, target gene - miRNA regulatory network and target gene - TF regulatory network of the DEGs. Expression levels of these candidate genes were finally verified by survival analysis, expression analysis( based on sample type and patients age), mutation analysis and immune histochemical (IHC) analysis, ROC (Receiver operating characteristic) curve analysis, RT-PCR and immune infiltration analysis. Overall, our systematic analysis will gain insights into GBM pathogenesis at molecular level and help to identify the potential candidate biomarkers for diagnosis, prognosis, and drug targets for GBM.

\section{Materials and methods}

\section{Selection of GEO data set}

Firstly, GBM-related chips GSE116520 [17] were retrieved and downloaded from the Gene Expression Omnibus (GEO) database (https://www.ncbi.nlm.nih.gov/geo/) with "Glioblastoma multform" serving as the retrieval key word. GSE116520 included eight normal control samples (brain) and seventeen GBM samples. The microarray platform was GPL10558 Illumina HumanHT-12 V4.0 expression beadchip Array. Flow chart of complete studies is shown in Fig. 1.

\section{Data pre-processing}

The downloaded probe-level raw data in TXT files were preprocessed using beadarray package [18] in $\mathrm{R}$ (version 3.3.2), including log-transformation, imputation of missing values, background correction, and quantile normalization. 
medRxiv preprint doi: https://doi.org/10.1101/2020.12.21.20248616; this version posted December 23, 2020. The copyright holder for this preprint (which was not certified by peer review) is the author/funder, who has granted medRxiv a license to display the preprint in perpetuity. All rights reserved. No reuse allowed without permission.

While several probes mapped to one gene, equate value of this probes was determined and used as the final expression value.

\section{Differential expression analysis}

The DEGs between GBM tissues and normal control tissues in each individual experiment were identified using Bayes moderated t-test method based on limma package [19], with the threshold criteria of FDR (false discovery rate) $<0.05$, $\mid \log 2 \mathrm{FC}$ (fold change) $\mid>1.88$ for up regulated genes and $\mid \log 2 \mathrm{FC}$ (fold change) $\mid<$ - 2.25 for down regulated genes. The relationships between samples and DEGs were shown by hierarchical clustering heatmaps and volcano plots.

\section{Pathway enrichment analysis of DEGs}

The pathway enrichment analyses were performed by ToppGene (ToppFun) (https://toppgene.cchmc.org/enrichment.jsp) [20]. BIOCYC (https://biocyc.org/) [21], Kyoto Encyclopedia of Genes and Genomes (KEGG; http://www.genome.jp/kegg/) [22], Pathway Interaction Database (PID, http://pid.nci.nih.gov/) [23], Reactome (https://reactome.org/PathwayBrowser/) [24], Molecular signatures database (MSigDB, http://software.broadinstitute.org/gsea/msigdb/) [25], GenMAPP (http://www.genmapp.org/) [26], Pathway Ontology (https://bioportal.bioontology.org/ontologies/PW) [27], PantherDB (http://www.pantherdb.org/) [28] and Small Molecule Pathway Database (SMPDB) (http://smpdb.ca/) [29] pathway enrichment analysis were carried out for the DEGs, with a $\mathrm{P}<0.05$ considered to indicate statistical significance.

\section{Gene ontology enrichment analysis of DEGs}

To explore the biological functional roles of the above DEGs, a GO (http://www.geneontology.org/) [30] enrichment analysis was performed on ToppGene (ToppFun) (https://toppgene.cchmc.org/enrichment.jsp) [20]. Significant results of biological process (BP), cellular component (CC) and molecular function (MF) with a cut-off of false discovery rate $<0.05$ were selected.

\section{PPI network construction and module analysis}


medRxiv preprint doi: https://doi.org/10.1101/2020.12.21.20248616; this version posted December 23, 2020. The copyright holder for this preprint (which was not certified by peer review) is the author/funder, who has granted medRxiv a license to display the preprint in perpetuity. All rights reserved. No reuse allowed without permission.

To further investigate the molecular mechanism of GBM, all DEGs were used to construct the PPI network using the biological online database tool (Integrated Interactions Database, IID, http://iid.ophid.utoronto.ca/) [31] to determine and predict the interaction among them. This database integrates various PPI data bases such as Biological General Repository for Interaction Datasets (BioGRID, https://thebiogrid.org/) [32], IntAct (https://www.ebi.ac.uk/intact/) [33], I2D (http://ophid.utoronto.ca/ophidv2.204/) [34], Molecular INTeraction database (MINT, https://mint.bio.uniroma2.it/) [35], InnateDB [https://www.innatedb.com/] [36], Database of Interacting Proteins (DIP, https://dip.doe-mbi.ucla.edu/dip/Main.cgi) [37], Human Protein Reference Database (HPRD, http://www.hprd.org/) [38] and the Biomolecular Interaction Network Database (BIND, http://bind.ca) [39]. A combined score $>0.7$ (high confidence score) was considered significant, and then the PPI network was visualized using Cytoscape software (http://www.cytoscape.org/) (Version 3.7.2) [40]. To evaluate the importance of nodes in the PPI network, the degree centrality, betweenness centrality, stress centrality, closeness centrality and clustering coefficient of nodes were calculated and utilized in the present study [41-45] using the network analyzer plugin in Cytoscape software. The hub genes, a small number of important nodes for the protein interactions in the PPI network, were chosen with a degree centrality $>50$, betweenness centrality $>0.02$, stress centrality $>$ 2100000, closeness centrality $>0.26$ and clustering coefficient $=0$. Because a higher k-core score means a more topological central location, modules in the PPI network were explored by k-core scoring using the PEWCC1 plugin in Cytoscape software [46], and significant modules with a k-core $>6$ were considered potential core regulatory networks.

\section{Construction of target gene - miRNA regulatory network}

To identify regulatory miRNAs that influence target gene (i.e., up and down regulated genes) at the posttranscriptional level, target gene - miRNA interactions were obtained from DIANA-TarBase (http://diana.imis.athenainnovation.gr/DianaTools/index.php? $r=$ tarbase/index) [47] and miRTarBase (http://mirtarbase.mbc.nctu.edu.tw/php/download.php) [48] both of which include experimentally supported target gene - miRNA interactions and topological parameter (degree) were analyzed using NetworkAnalyst (https://www.networkanalyst.ca/) [49]. 
medRxiv preprint doi: https://doi.org/10.1101/2020.12.21.20248616; this version posted December 23, 2020. The copyright holder for this preprint (which was not certified by peer review) is the author/funder, who has granted medRxiv a license to display the preprint in perpetuity. All rights reserved. No reuse allowed without permission.

\section{Construction of target gene - TF regulatory network}

To identify regulatory TFs that control the i.e., up and down regulated genes) at a transcriptional level, TF-target gene interactions were obtained using the ChEA database (http://amp.pharm.mssm.edu/lib/chea.jsp) [50] and were identified topological parameter (degree centrality) using (https://www.networkanalyst.ca/) [49].

\section{Validation of hub genes and clinical significance}

The UALCAN (https://ualcan.path.uab.edu/index.html) [51] online database was used for survival analysis, expression analysis and age related expression analysis of the hub genes, which analyzed RNA sequencing expression data from TCGA projects. The mutation frequency of hub genes was inquired in cBioportal online database (http://www.cbioportal.org/) [52]. The hub gene expressions in GBM tissues were determined from the human protein atlas (www.proteinatlas.org) [53]. To explore diagnostic biomarkers of GBM, we used the above hub genes as candidates to find their diagnostic value based on generalized linear models (GLM). The pROC package [54] in R was used for GLM analysis. In brief, half of the samples $(\mathrm{GBM}=17$, controls $=8)$ were randomly distributed as the training set, which was used to build a model. An ROC (Receiver operating characteristic) curve analysis was practiced to calculate the specificity and sensitivity of the GLM prediction model. The AUC was computed to evaluate the diagnostic efficiency of the classifier. All cell culture samples of normal (HCN-1A) and GBM (U-118 MG) were lysed using TRIzol® (Invitrogen; Thermo Fisher Scientific, Inc.), and total RNAs were extracted and reverse transcribed into cDNA templates using PrimeScript ${ }^{\circledR}$ RT Reagent kit (Takara Biotechnology Co., Ltd.) according to the manufacturer's instructions. PCR was performed using an $7900 \mathrm{HT}$ real-time PCR instrument with an initial denaturation at $95{ }^{\circ} \mathrm{C}$ for $30 \mathrm{~s}$, followed by 40 cycles at $95{ }^{\circ} \mathrm{C}$ for $15 \mathrm{~s}$ and $60{ }^{\circ} \mathrm{C}$ for $34 \mathrm{~s}$, and a fnal dissociation curve analysis of one cycle at $95{ }^{\circ} \mathrm{C}$ for $15 \mathrm{~s}, 60{ }^{\circ} \mathrm{C}$ for $1 \mathrm{~min}$, and $95{ }^{\circ} \mathrm{C}$ for $15 \mathrm{~s}$. Each cDNA sample was assayed three times and relative expression was resolved using the $2^{-\triangle \Delta C T}$ method [55]. The specific PCR primers for the hub genes and $\beta$-actin as the internal control gene were designed with Primer Express version 2.0. TIMER 
medRxiv preprint doi: https://doi.org/10.1101/2020.12.21.20248616; this version posted December 23, 2020. The copyright holder for this

(https://cistrome.shinyapps.io/timer/) [56] is a user friendly, interactive web resource for immune infiltration analysis from RNA-Seq expression profiling database (The Cancer Genome Atlas (TCGA)). Immune infiltration analysis was evaluated using immune infiltrates (B cells, CD4+ T cells, CD8+ T cells, neutrophils, macrophages, and dendritic cells) across GBM.

\section{Results}

\section{Data preprocessing and screening of DEGs}

The gene expression profile GSE116520 was downloaded from the GEO. The data before and after normalization are shown in Fig. 2A and Fig. 2B. The limma method was used to identify DEGs in GBM tissue compared with normal control tissues (brain). $\mathrm{P}$ value $<0.05, \log \mathrm{FC}>1.88$ for up regulated genes, and $\log \mathrm{FC}<$ - 2.25 for down regulated genes were used as the cut-off criteria. After analyzing, total of 947 DEGs were selected between the GBM tissues and normal control tissues, including 477 up genes and 470 down regulated genes (Table 1). The result is displayed in the volcano plot (Fig. 3). The heatmap of the DEGs (up and down regulated genes) are shown in Fig. 4 and Fig. 5.

\section{Pathway enrichment analysis of DEGs}

In order to investigate the biological functions of these DEGs (up and down regulated genes) in GBM. Pathway enrichment analysis was performed using ToppGene. Pathway enrichment analysis results indicated that DEGs (up and down regulated genes) were significantly enriched in reactive oxygen species degradation, glutamate removal from folates, ribosome, cell cycle, FOXM1 transcription factor network, PLK1 signaling events. translation, extracellular matrix organization, starch and sucrose_metabolism, nitrogen_metabolism, ensemble of genes encoding core extracellular matrix including ECM glycoproteins, collagens and proteoglycans, ensemble of genes encoding extracellular matrix and extracellular matrix-associated proteins, integrin signalling pathway, p53 pathway, hypertension, G2/M DNA replication checkpoint, and nicotinate and nicotinamide metabolism, homocarnosine biosynthesis, fatty acid alpha-oxidation III, GABAergic synapse, insulin secretion, effects of botulinumtoxin, internalization of ErbB1, neuronal system, transmission across chemical synapses, alanine and aspartate metabolism, glycans biosynthesis, 
medRxiv preprint doi: https://doi.org/10.1101/2020.12.21.20248616; this version posted December 23, 2020. The copyright holder for this

Wnt/Ca2+/cyclic GMP signaling., fl-arrestins in GPCR desensitization, synaptic vesicle trafficking, muscarinic acetylcholine receptor 1 and 3 signaling pathway, insulin secretion pathway, glutamate metabolic, pirenzepine pathway and homocarnosinosis are listed in Table 2 and Table 3.

\section{Gene ontology enrichment analysis of DEGs}

GO enrichment analysis was conducted using the ToppGene, and the results are illustrated in Table 4 and Table 5. For up regulated genes, the terms enriched in the BP category included macromolecule catabolic process and mitotic cell cycle. The GO CC category revealed enrichment in the cytosolic part and collagen-containing extracellular matrix. In addition, the MF category showed enrichment for factors involved in structural molecule activity and RNA binding. Down regulated genes showed enrichment in the BP category in processes such as synaptic signaling and cell-cell signaling. The enriched terms in the CC category mainly included synapse part and neuron projection. Additionally, the enriched MF was focused on ion gated channel activity and channel activity.

\section{PPI network construction and module analysis}

There were 4162 nodes and 8776 relation pairs in PPI network for up regulated genes (Fig. 6). Hub genes in the network were analyzed, and the top nodes with the highest degree, betweenness centrality, stress centrality, closeness centrality score and lowest clustering coefficient score were MYC, VCAM1, CDK2, HNRNPA1, PCNA, CDK1, EEF1A1, HSPD1, HNRNPK, CEP55, A2M, CDCA5, ETS1 and PTGES3 are listed Table 6. The statistical results and scatter plot for node degree distribution, betweenness centrality, stress centrality, closeness centrality and clustring coefficient are shown in Fig. 7. These hub genes were enriched in cell cycle, TNF signaling pathway, FOXM1 transcription factor network, processing of capped intron-containing pre-mRNA, macromolecule catabolic process, mitotic cell cycle, regulation of cell death, validated targets of $\mathrm{C}$ MYC transcriptional activation, metabolism of proteins, microtubule cytoskeleton, complement and coagulation cascades, protein-containing complex binding, pathways in cancer and C20 prostanoid biosynthesis. Similarly, there were 2392 nodes and 3196 relation pairs in PPI network for down regulated genes (Fig. 8). Hub genes in the network were analyzed, and the top nodes with the highest degree 
medRxiv preprint doi: https://doi.org/10.1101/2020.12.21.20248616; this version posted December 23, 2020. The copyright holder for this preprint (which was not certified by peer review) is the author/funder, who has granted medRxiv a license to display the preprint in perpetuity. All rights reserved. No reuse allowed without permission.

score were ARRB1, SNCA, ERBB3, PRKCZ, DLG4, SLC30A3, DNM1, FAM153B, RAPGEF5, EFHD1, PDYN, ZNF536 and TSPOAP1 are listed Table 6. . The statistical results and scatter plot for node degree distribution, betweenness centrality, stress centrality, closeness centrality and clustring coefficient are shown in Fig. 9. These hub genes were enriched in endocytosis, Parkinson's disease, calcium signaling pathway, synaptic signaling, glutamatergic synapse, transmembrane transport of small molecules, synaptic vesicle cycle, neurogenesis, signaling by GPCR, neuron differentiation and neuronal system.

Subsequently, we performed module analysis of the whole network by the PEWCC1 plug-in. Total 849 modules were identified in PPI network for up regulated genes. Those hub genes were located at module 6 , module 15, module 24 and module 51, are the most informative modules in PPI analysis (Fig. 10). These significant modules were proven to be associated with different pathways and GO categories such as ribosome, cell cycle, TNF signaling pathway, pathways in cancer, macromolecule catabolic process, mitotic cell cycle, RNA binding and cytosolic part. Similarly, total 201 modules were identified in PPI network for down regulated genes. Those hub genes were located at module 2 , module 7 , module 18 and module 22, are the most informative modules in PPI analysis (Fig. 11). These significant modules were proven to be associated with different pathways and GO categories such as insulin secretion, synaptic vesicle cycle, glutamatergic synapse, endocytosis, synaptic signaling, neurogenesis, cell-cell signaling and neuron differentiation.

\section{Construction of target gene - miRNA regulatory network}

For further research of the target genes (up and down regulated genes), target generelated miRNAs were predicted by DIANA-TarBase and miRTarBase. Main miRNAs with interactions of target genes are listed in Table 7. Target genes were found to play a key role in regulating miRNAs. The target genes - miRNA regulatory network (up regulated genes) included 2440 nodes and 8546 edges (Fig.12). SOD2 was predicted to regulate 257 miRNAs (eg, hsa-mir-6077), WEE1 was predicted to regulate 167 miRNAs (eg, hsa-mir-4457), G3BP1 was predicted to regulate 158 miRNAs (eg, hsa-mir-4457), CNBP was predicted to regulate 153 miRNAs (eg, hsa-mir-4260) and HMGB1 was predicted to regulate 143 miRNAs (eg, hsa-mir-5193). These target genes were enriched in reactive oxygen species 
medRxiv preprint doi: https://doi.org/10.1101/2020.12.21.20248616; this version posted December 23, 2020. The copyright holder for this

degradation, cell cycle, adherens junction, RNA binding and Neutrophil degranulation. The target genes - miRNA regulatory network (down regulated genes) included 2046 nodes and 4596 edges ((Fig.13). SVOP was predicted to regulate 107 miRNAs (eg, hsa-mir-3972), KCNJ6 was predicted to regulate 90 miRNAs (eg, hsa-mir-4287), SYT7 was predicted to regulate 75 miRNAs (eg, hsamir-4441), RAB11FIP4 was predicted to regulate 73 miRNAs (eg, hsa-mir-3176) and NPTX1 was predicted to regulate 73 miRNAs (eg, hsa-mir-3119). These target genes were enriched in transmembrane transport, synapse part, neuronal system, endocytosis and synaptic signaling.

\section{Construction of target gene - TF regulatory network}

For further research of the target genes (up and down regulated genes), target generelated TFs were predicted by ChEA database. Main TFs with interactions of target genes are listed in Table 8. Target genes were found to play a key role in regulating TFs. The target genes - TF regulatory network (up regulated genes) included 555 nodes and 9100 edges (Fig.14). ABCC3 was predicted to regulate 225 TFs (eg, SOX2), VKORC1 was predicted to regulate 180 TFs (eg, NANOG), MCTS1 was predicted to regulate $171 \mathrm{TFs}$ (eg, SPI1), TNFRSF12A was predicted to regulate 167 TFs (eg, E2F1) and C15orf48 was predicted to regulate 155 TFs (eg, POU5F1). These target genes were enriched in whole membrane, cell cycle and cytokine signaling in immune system. The target genes - TF regulatory network (down regulated genes) included 576 nodes and 8171 edges (Fig.15). ABCA2 was predicted to regulate 234 TFs (eg, SUZ12), MOBP was predicted to regulate 201 TFs (eg, REST), PLEKHG3 was predicted to regulate 198 TFs (eg, EGR1), TTLL7 was predicted to regulate 188 TFs (eg, SOX2) and CAPN3 was predicted to regulate $178 \mathrm{TFs}$ (eg, AR). These target genes were enriched in transmembrane transport of small molecules, cytoskeletal protein binding, neuron projection and Huntington disease.

\section{Validation of hub genes and clinical significance}

UALCAN, the online tool with data sourced from TCGA, was used to validate the expression of these hub genes in GBM. Survival analysis $(\mathrm{P}<0.05)$ (Fig. 16); highly expressing TUBA1C, CAV1, S100A4, DNAJA4, PAK6, NELL1 and ITPKA tends to have poor survival outcomes in GBM. However, low expressing 
medRxiv preprint doi: https://doi.org/10.1101/2020.12.21.20248616; this version posted December 23, 2020. The copyright holder for this preprint (which was not certified by peer review) is the author/funder, who has granted medRxiv a license to display the preprint in perpetuity. All rights reserved. No reuse allowed without permission.

RPL23, YY1 and ARHGEF7 tends to have poor survival outcomes in GBM. As shown in Fig 17, the expression of the up regulated hub genes TUBA1C, CAV1, RPL23, YY1 and S100A4 in GBM tissue were significantly elevated compared with normal brain tissues. Furthermore, the expressions of down regulated hub genes ARHGEF7, DNAJA4, PAK6, NELL1 and ITPKA in GBM tissue were significantly decreased compared with normal brain tissues. The expression of each hub gene in GBM patients was analyzed according to the patient's age. As shown in Fig 18, the expression of TUBA1C, CAV1, RPL23, YY1 and S100A4 were higher in patients with age (21-40 years, 21-60 years, 61-80 years, 81-100 years), which revealed that these up regulated hub genes might be associated with GBM advancement positively. Similarly, the expression of ARHGEF7, DNAJA4 , PAK6, NELL1 and ITPKA were lower in patients age (21-40 years, 21-60 years, 61-80 years, 81-100 years), which revealed that these down regulated hub genes might be linked with GBM advancement positively. Fig. 19 presented the mutation information of the ten hub genes. TUBA1C, CAV1, RPL23, YY1, S100A4, ARHGEF7, DNAJA4, PAK6, NELL1 and ITPKA were changed most often $(0.7 \%, 0.4 \%, 0.4 \%, 0.7 \%, 0.4 \%, 0.7 \%, 0.4 \%, 0.7 \%, 1.5 \%$ and $0.4 \%)$, these include amplification, deep deletion, missense mutation and truncating mutation. The Human Protein Atlas (THPA) demonstrated that the expression of TUBA1C, CAV1, RPL23, YY1 and S100A4 were highly expressed in GBM tissues, whereas ARHGEF, DNAJA4, PAK6, NELL1 and ITPKA were low expressed in GBM tissue (Fig. 20). To verify the diagnostic value of the hub genes, expression levels in GBM were evaluated using ROC curves. As presented in Fig. 21, the area under the curve (AUC) for TUBA1C, CAV1, RPL23, YY1, S100A4, ARHGEF7, DNAJA4, PAK6, NELL1 and ITPKA in GBM and normal control tissue determined for the GSE116520 dataset were 0.963, 0.971, 0.993, 0.963, 0.971, $0.963,0.985,0.971,0.978$ and 0.985 , respectively. RT-PCR demonstrated that the relative expression levels of TUBA1C, CAV1, RPL23, YY1 and S100A4 in GBM tissues were significantly higher compared with those in normal tissue (Fig. 22A - E), whereas expression levels of ARHGEF7, DNAJA4, PAK6, NELL1 and ITPKA in GBM tissue were significantly lower compared with those in normal tissue (Fig. 22 F- J). The PCR primers are listed in Table 9. To investigate the immune infiltration analysis of the ten potential hub genes, the TIMER bioinformatics analysis platform was used. We found that the high expression of hub genes (TUBA1C, CAV1, RPL23, YY1 and S100A4) were negatively 
medRxiv preprint doi: https://doi.org/10.1101/2020.12.21.20248616; this version posted December 23, 2020. The copyright holder for this

associated with tumor purity (Fig. 23A - E), where as low expression of hub genes (ARHGEF7, DNAJA4, PAK6, NELL1 and ITPKA) were positively associated with tumor purity (Fig. 23A - E).

\section{Discussion}

The majorities of patients with GBM are diagnosed at advanced stages and have poor overall survival [57]. However, the molecular mechanisms associated in the advancement of GBM remain unclear. In the present study, to better understanding the molecular mechanisms involved in GBM progression, we characterized the expression profiles in GBM and normal brain tissues by transcriptome analysis. Using bioinformatics analysis, we obtained 947 DEGs from GSE116520 data expression profiles, including 477 up regulated genes and 470 down regulated genes. Genes such as SERPINA3 [58], VIP (vasoactive intestinal peptide) [59], ANXA2 [60] and SST (somatostatin) [61] were associated with progression of GBM. RPL39 [62] was responsible for invasion of breast cancer cells, but this gene was identified first time in GBM and may be linked with invasion of GBM cells. Genes such as TUBA1 [63], RPN2 [64], RASAL1 [65] and CCKBR (cholecystokinin $\mathrm{B}$ receptor) [66] were involved in proliferation of different cancer cells, but expression of these genes are not reported in GBM and may be associated with proliferation of GBM cells. High expression of KLK7 was important for pathogenesis of ovarian cancer [67], but elevated expression of this gene was identified first time in GBM and may be liable for progression of GBM.

In pathway enrichment analysis for up regulated genes was performed. Enriched genes such as SOD2 [68], RPS11 [69], RPL9 [70], MYC (MYC protooncogene, bHLH transcription factor) [71], SEC61G [72], BIRC5 [73], NEK2 [74], CDK2 [75], AURKB (aurora kinase B) [76], RPS3 [77], MGP (matrix Gla protein) [78], AEBP1 [79], CTHRC1 [80], COL1A1 [81], COL3A1 [82], TNC (tenascin C) [83], POSTN (periostin) [84], IGFBP2 [85], IGFBP3 [86], IGFBP4 [87], SRPX2 [88], LAMB1 [89], ESM1 [90], TGFBI (transforming growth factor beta induced) [91], ITGA5 [92], RAP1B [93], CAV1 [94], HMOX1 [95] and LOX (lysyl oxidase) [96] were linked with progression of GBM. GPX7 was important for advancement of gastric cancer [97], but this gene was identified first time in GBM and may be liable for progression of GBM. High expression of enriched genes such as RPL29 [98], RPLP1 [99], RPS2 [100], RPS3A [101], RPS13 [102], 
medRxiv preprint doi: https://doi.org/10.1101/2020.12.21.20248616; this version posted December 23, 2020. The copyright holder for this preprint (which was not certified by peer review) is the author/funder, who has granted medRxiv a license to display the preprint in perpetuity. All rights reserved. No reuse allowed without permission.

RPS15A [103], RPL7A [104], CENPA (centromere protein A) [105], CENPF (centromere protein F) [106], EIF4E [107], MXRA5 [108] and LUM (lumican) [109] were responsible for development of different cancer types, but over expression of these genes were identified first time in GBM and may be associated with development of GBM. Enriched genes such as RPS12 [110], RPL6 [111], LAMA4 [112], CCNB1 [113], CCNB2 [114], CDK1 [115] and EIF3M [116] were responsible for proliferation of different cancer cell types, but these genes were identified first time in GBM and may be associated with proliferation of GBM cells. Enriched polymorphic genes such as RPL14 [117] and LAMC1 [118] were liable for advancement of different cancer types, but these polymorphic genes were identified first time in GBM and may be important for development of GBM. Enriched genes associated such as RPL15 [119], EEF1A1 [120], SRPX (sushi repeat containing protein X-linked) [121], COL1A2 [122], COL4A1 [123], COL5A1 [124], COL5A2 [125], COL6A3 [122] and COL8A1 [126] were involved in invasion of different cancer cell types, but these genes were identified first time in GBM and may be culpable for invasion of GBM cells. EMILIN2 was associated with angiogenesis in gastric cancer [127], but this gene was identified first time in GBM and may be important for angiogenesis in GBM. Our study found that GPX8, RPL23A, RPL31, RPS4X, RPS4Y1, RPS7, RPS8, RPS10, RPS18, RPS25, RPS26, RPS27, RPS27A, RPS28, RPS29, RPL23, RPL7, RPL18A, EEF1B2, AMY1A, GBE1, PYGL (glycogen phosphorylase L), PCOLCE2, TNFAIP6 and SLC7A6 are up regulated in GBM and has potential as a novel diagnostic and prognostic biomarker, and therapeutic target. Similarly, pathway enrichment analysis for down regulated genes was performed. Enriched genes such as SLC12A5 [128], SHANK2 [129], KCNJ4 [130] and CACNA1E [131] were linked with development of different cancer types, but these genes were identified first time in GBM and may be important for progression of GBM. Enriched genes such as SLC6A1 [132], GABBR1 [133] and GAD1 [134] were associated with invasion of different cancer cells, but these genes were identified first time in GBM and may be involved in invasion of GBM cells. Enriched genes such as GLS (glutamines) [135], NEFL (neurofilament light) [136], SYN1 [137], SLC17A7 [138], SYT7 [139], EPB41L1 [140] and TF (transferrin) [141] were responsible for advancement of GBM. Methylation inactivation of enriched tumor suppressor genes such as such as GNAO1 [142], KCNMA1 [143] and CAMK2B [144] were liable for progression of different cancer types, but these genes were 
medRxiv preprint doi: https://doi.org/10.1101/2020.12.21.20248616; this version posted December 23, 2020. The copyright holder for this preprint (which was not certified by peer review) is the author/funder, who has granted medRxiv a license to display the preprint in perpetuity. All rights reserved. No reuse allowed without permission.

identified first time in GBM and loss of these genes may be linked with development of GBM. Low expression of UNC13C was associated with development of oral cancer [145], but this gene was identified first time in GBM and decrease expression of this gene may be linked with progression of GBM. Polymorphic gene CHRM3 was identified with progression of bladder cancer [146], but this polymorphic gene was identified first time in GBM and may be liable for advancement of GBM. Our study found that CARNS1, ADCY1, ADCY2, GABBR2, SLC32A1, PRKCB (protein kinase C beta), GABRA2, GABRA5, GABRB1, GABRB3, GABRG2, GAD2, KCNJ6, GNG3, SNAP25, STX1A, STXBP1, SYT1, DLGAP2, TSPOAP1, CACNG3, PPFIA2, SLC1A2, SHANK3, CPLX1, KCNK4, PTPRD (protein tyrosine phosphatase receptor type D), ABCC8, SYN2, KCNAB1, KCNQ2, KCNQ3, KCNS1, DLG2, DLG4, CAMK2A, GRIN1, GRIN2C, KCNH3, ASPA (aspartoacylase), ITPKA (inositoltrisphosphate 3-kinase A), STX1B, RIMS2 and SYP (synaptophysin) are down regulated in GBM and has potential as a novel diagnostic and prognostic biomarker, and therapeutic target.

In GO enrichment analysis for up regulated genes was performed. Enriched genes such as PTTG1 [147], HMGB1 [148], HMGB2 [149], HMMR (hyaluronan mediated motility receptor) [150], CHI3L2 [151], VEGFA (vascular endothelial growth factor A) [152], VIM (vimentin) [153], IGF2BP3 [154], UHRF1 [155], SUMO2 [156], PBK (PDZ binding kinase) [157], AURKA (aurora kinase A) [158], ADAMTS9 [159], UBE2C [160], CAST (calpastatin) [161], USP8 [162], TIMP1 [163], TIMP4 [164], CD44 [165], PCNA (proliferating cell nuclear antigen) [166], CCT8 [167], CHI3L1 [168] and ANXA1 [169] were involved in progression of GBM. HNRNPC (heterogeneous nuclear ribonucleoprotein C) was associated with drug resistance in gastric cancer [170], but this gene was identified first time in GBM and may be associated with chemo resistance in GBM. Enriched genes such as HSPA1A [171] and TUBA1C [172] were linked with proliferation of liver cancer cells, but these genes were identified first time in GBM and may be involved in proliferation of GBM cells. High expression of enriched genes such as MAD2L1 [173] and CSRP2 [174] were linked with pathogenesis of different cancer types, but high expression of these genes were identified first time in GBM and may be involved in progression of GBM. CASP4 [175] was involved in advancement of esophageal cancer, but this gene was identified first time in GBM 
medRxiv preprint doi: https://doi.org/10.1101/2020.12.21.20248616; this version posted December 23, 2020. The copyright holder for this preprint (which was not certified by peer review) is the author/funder, who has granted medRxiv a license to display the preprint in perpetuity. All rights reserved. No reuse allowed without permission.

and may be associated in development of GBM. Our study found that LSM5, CPVL (carboxypeptidasevitellogenic like), PPP2CB, CYP51A1, BNIP3L, FBXO5, ZFP36L1, RNASE2, MCTS1, LARP4, PRPH (peripherin), POTEKP (POTE ankyrin domain family member $\mathrm{K}$, pseudogene), TUBB6, ACTR3 and RNA28SN5 are up regulated in GBM and has potential as a novel diagnostic and prognostic biomarker, and therapeutic target. Similarly, GO enrichment analysis for down regulated genes was performed. Enriched genes such as MAG (myelin associated glycoprotein) [176], ASIC2 [177], MBP (myelin basic protein) [178], CNP (2',3'cyclic nucleotide 3' phosphodiesterase) [179], CPEB3 [180], SLC8A2 [181], PRKCZ (protein kinase C zeta) [182], RELN (reelin) [183], CYP46A1 [184], SNAP91 [185], CNTN2 [186], NPY (neuropeptide Y) [187], RGS4 [188], IL1RAPL1 [189], ERBB3 [190], SH3GL2 [191], SH3GL3 [192], ARRB1 [193], DNM3 [194], SPOCK1 [195], CCK (cholecystokinin) [196] and INA (internexin neuronal intermediate filament protein alpha) [197] were identified with progression of GBM. Decrease expression of enriched genes such as such as SCN8A [198], BRSK1 [199], ANKS1B [200], CALB2 [201], GRM3 [202], BCAS1 [203] and CLCA4 [204] were responsible for advancement of different cancer types, but low expression of these genes were identified first time in GBM and may be involved in progression of GBM. Enriched genes such as CUX2 [205], NPTX1 [206], NCS1 [207], SEPTIN4 [208] and FAIM2 [209] were associated with advancement of different cancer, but these genes were identified first time in GBM and may be linked with development of GBM. MAP4 was involved in invasion of bladder cancer cells [210], but this gene was identified first time in GBM and may be responsible for invasion of GBM cells. Enriched genes such as RAB6B [211] and MAL2 [212] were linked with proliferation of different cancer cells types, but these genes were identified first time in GBM and may be liable for proliferation of GBM cells. Methylation inactivation of tumor suppressor DMTN (dematin actin binding protein) was associated with progression of colorectal cancer [213], but loss of this gene was identified first time in GBM and may be involved in advancement of GBM. Our study found that MAP1A, PDYN (prodynorphin), TMOD2, CPNE6, SCN2A, SCN2B, FGF12, PLP1, AMPH (amphiphysin), HTR2A, NSG2, NAPB (NSF attachment protein beta), CNTNAP2, CNTNAP4, CALY (calcyon neuron specific vesicular protein), ERC2, SNCA (synuclein alpha), ATP2B2, JPH4, RIMS3, CDK5R1, SV2B, SYT4, CACNA1I, BSN (bassoon presynaptic cytomatrix protein), DNM1, NRGN (neurogranin), 
medRxiv preprint doi: https://doi.org/10.1101/2020.12.21.20248616; this version posted December 23, 2020. The copyright holder for this

PHF24, PCLO (piccolo presynaptic cytomatrix protein), RAPGEF4, NETO1, SYNGR1, RIMBP2, LY6H, JPH3, PDE2A, KCNIP3, SYNPR (synaptoporin), SLITRK1, HPCA (hippocalcin), CAMKV (CaM kinase like vesicle associated), KCTD16, PPP1R1B, OLFM1, SVOP (SV2 related protein), PACSIN1, PKP4, MAGEE1, SH2D5, LGI3, ATP6V1G2, KIF1A, SLC6A17, DDN (dendrin), LAMP5, SLC30A3, NEFM (neurofilament medium), SEPTIN3, ARHGAP44, KIAA1107, RGS7BP, RGS7, KCNT1, KCNK12, PEX5L, ANO3, SCN3B and ANO4 are down regulated in GBM and has potential as a novel diagnostic and prognostic biomarker, and therapeutic target.

The up regulated hub genes obtained from PPI network. Hub genes such as VCAM1 [214], HNRNPA1 [215], CEP55 [216], A2M [217] and ETS1 [218] were responsible for advancement of GBM. HSPD1 was associated with proliferation of breast cancer cells [219], but this gene was identified first time in GBM and may be linked with proliferation of GBM cells. HNRNPK (heterogeneous nuclear ribonucleoprotein K) was liable for invasion of nasopharyngeal cancer cells [220], but this gene was identified first time in GBM and may be involved in invasion of GBM cells. High expression of CDCA5 was identified with development of esophageal cancer [221], but elevated expression of this gene was identified first time in GBM and may be linked with advancement of GBM. Our study found that PTGES3 is up regulated in GBM and has potential as a novel diagnostic and prognostic biomarker, and therapeutic target. The down regulated hub genes obtained from PPI network. Methylation inactivation of EFHD1 was liable for development of colorectal cancer [222], but loss of this gene was identified first time in GBM and may be responsible for progression of GBM. Our study found that FAM153B, RAPGEF5 and ZNF536 are down regulated in GBM and has potential as a novel diagnostic and prognostic biomarker, and therapeutic target.

Significant modules were extracted from PPI network to obtain up regulated hub genes. Hub genes such as CDKN3 [223], CCNA2 [224] and CKS2 [225] were responsible for proliferation of different cancer cells types, but these genes were identified first time in GBM and may be associated with proliferation of GBM cells. Over expression of GMNN (geminin DNA replication inhibitor) was linked with progression of liver cancer [226], but high expression of this gene was 
medRxiv preprint doi: https://doi.org/10.1101/2020.12.21.20248616; this version posted December 23, 2020. The copyright holder for this

identified first time in GBM and may be liable for advancement of GBM. KPNA2 was involved in progression of GBM [227]. Our study found that CNBP (CCHCtype zinc finger nucleic acid binding protein) and NSMAF (neutral sphingomyelinase activation associated factorÂ) are up regulated in GBM and has potential as a novel diagnostic and prognostic biomarker, and therapeutic target.. Similarly, significant modules were extracted from PPI network to obtain down regulated hub genes. ARHGEF7 was linked with invasion of colorectal cancer cells [228], but this gene was identified first time in GBM and may be liable for invasion of GBM cells.

Target gene - miRNA regulatory network was constructed for up and down regulated genes. Target genes such as WEE1 [229] and G3BP1 [230] were responsible for development of GBM. RAB11FIP4 was linked with invasion of colon cancer cells [231], but this gene was identified first time in GBM and may be liable for invasion of GBM cells.

Target gene - TF regulatory network was constructed for up and down regulated genes. Target genes such as ABCC3 [232] and ABCA2 [233] were involved in progression of GBM. High expression of TNFRSF12A was liable for advancement of breast cancer [234], but elevated expression of this gene was identified first time in GBM and may be involved in development of GBM. C15orf48 (NMES1) was associated with development of esophageal cancer [235], but this gene was identified first time in GBM and may be identified with growth of GBM. Up and down regulated genes such as VKORC1, MOBP (myelin associated oligodendrocyte basic protein), PLEKHG3, TTLL7 and CAPN3 were associated in target gene - TF regulatory network and were identified as novel biomarker for pathogenesis of GBM.

High expression of hub genes (TUBA1C, CAV1, S100A4, DNAJA4, PAK6, NELL1 and ITPKA) were significantly associated with poor overall survival (OS) in GBM, while low expression of hub genes (RPL23, YY1 and ARHGEF7) were significantly associated with poor over OS in GBM and were visualized using UALCAN. Genes such as S100A4 [236] and YY1 [237] were responsible for progression of GBM. PAK6 was linked with proliferation of lung cancer cells [238], but this gene was identified first time in GBM and may be involved in proliferation of GBM cells. Polymorphic gene NELL1 was liable for 
medRxiv preprint doi: https://doi.org/10.1101/2020.12.21.20248616; this version posted December 23, 2020. The copyright holder for this

progression of oral cancer [239], but this polymorphic gene was identified first time in GBM and may be linked with advancement of GBM. Next, the expression analysis of these hub genes in GBM compared with the normal and was verified on the UALCAN website. It was found that TUBA1C, CAV1, RPL23, YY1 and S100A4 were highly expressed in patients with GBM compared with normal people, while ARHGEF7, DNAJA4, PAK6, NELL1 and ITPKA were low expressed in patients with GBM compared with normal people. Next, the expression analysis of these hub genes in different age groups of GBM patients and was verified on the UALCAN website. All hub genes were showed altered expressed in all age groups of GBM patients and was verified on the UALCAN website. The mutation analysis found that mutations or alterations in all hub genes and was verified on the cBioportal website. All hub genes were validated by ICH analysis and was verified on the human protein atlas. Finally, all hub genes were validated by ROC analysis using pROC package in R software, RT-PCR and immune infiltration analysis.

In conclusion, we successfully diagnosed hub genes (TUBA1C, CAV1, RPL23, YY1, S100A4, ARHGEF7, DNAJA4, PAK6, NELL1 and ITPKA) based on bioinformatic analysis and experimental validation. This study shows that TUBA1C, CAV1, RPL23, YY1, S100A4, ARHGEF7, DNAJA4, PAK6, NELL1 and ITPKA plays a major role in the progression of GBM and has broad application potential.

\section{Acknowledgement}

I thank Ruchi Jain, Indian Institute of Science, Molecular Reproduction Development and Genetics, C V Raman Road, Bangalore, Karnataka, India, very much, the author who deposited their microarray dataset, GSE116520, into the public GEO database.

\section{Conflict of interest}

The authors declare that they have no conflict of interest.

\section{Ethical approval}

This article does not contain any studies with human participants or animals performed by any of the authors. 


\section{Informed consent}

No informed consent because this study does not contain human or animals participants.

\section{Availability of data and materials}

The datasets supporting the conclusions of this article are available in the GEO (Gene Expression Omnibus) (https://www.ncbi.nlm.nih.gov/geo/) repository. [(GSE116520)

(https://www.ncbi.nlm.nih.gov/geo/query/acc.cgi?acc=GSE116520)]

\section{Consent for publication}

Not applicable.

\section{Competing interests}

The authors declare that they have no competing interests.

\section{Author Contributions}

Basavaraj Vastrad : $\quad$ Writing original draft, and review and editing

Chanabasayya Vastrad: Investigation and resources

Iranna Kotturshetti : $\quad$ Supervision and resources

\section{Authors}

Basavaraj Vastrad

Chanabasayya Vastrad

Iranna Kotturshetti
ORCID ID: 0000-0003-2202-7637

ORCID ID: 0000-0003-3615-4450

ORCID ID: 0000-0003-1988-7345

\section{References}

1. Tran B, Rosenthal MA. Survival comparison between glioblastoma multiforme and other incurable cancers. Journal of Clinical Neuroscience. 2010;17(4):417-421. doi:10.1016/j.jocn.2009.09.004 
medRxiv preprint doi: https://doi.org/10.1101/2020.12.21.20248616; this version posted December 23, 2020. The copyright holder for this

2. Bray F, Ferlay J, Soerjomataram I, Siegel RL, Torre LA, Jemal A. Global cancer statistics 2018: GLOBOCAN estimates of incidence and mortality worldwide for 36 cancers in 185 countries. CA: a cancer journal for clinicians. 2018;68(6):394-424. doi:10.3322/caac.21492

3. Sarria GR, Sperk E, Han X, Sarria GJ, Wenz F, Brehmer S, Fu B, Min S, Zhang $\mathrm{H}$, Qin $\mathrm{S}$ et al. Intraoperative radiotherapy for glioblastoma: an international pooled analysis. Radiotherapy and Oncology. 2019. doi:10.1016/j.radonc.2019.09.023

4. Saito T, Muragaki Y, Maruyama T, Komori T, Nitta M, Tsuzuki S, Fukui A, Kawamata $T$. Influence of wide opening of the lateral ventricle on survival for supratentorial glioblastoma patients with radiotherapy and concomitant temozolomide-based chemotherapy. Neurosurgical Review. 2019 8:1-1. doi:10.1007/s10143-019-01185-2

5. Sezer S, van Amerongen MJ, Delye HH, ter Laan M. Accuracy of the neurosurgeons estimation of extent of resection in glioblastoma. Acta neurochirurgica. 2019 28:1-6. doi:10.1007/s00701-019-04089-8

6. Alshabi AM, Vastrad B, Shaikh IA, Vastrad C. Identification of Crucial Candidate Genes and Pathways in Glioblastoma Multiform by Bioinformatics Analysis. Biomolecules. 2019;9(5):201. doi:10.3390/biom9050201

7. Deng Y, Yao L, Chau L, Ng SS, Peng Y, Liu X, Au WS, Wang J, Li F, Ji S et al. N-Myc downstream-regulated gene 2 (NDRG2) inhibits glioblastoma cell proliferation. International journal of cancer. 2003;106(3):342-347. doi:10.1002/ijc.11228

8. Veeriah S, Taylor BS, Meng S, Fang F, Yilmaz E, Vivanco I, Janakiraman M, Schultz N, Hanrahan AJ, Pao W et al. Somatic mutations of the Parkinson's disease-associated gene PARK2 in glioblastoma and other human malignancies. Nature genetics. 2010;42(1):77-82. doi:10.1038/ng.491

9. Nakahara Y, Okamoto H, Mineta T, Tabuchi K. Expression of the Wilms' tumor gene product WT1 in glioblastomas and medulloblastomas. Brain tumor pathology. 2004;21(3):113-116. doi:10.1007/bf02482185

10.Nakamura M, Yonekawa Y, Kleihues P, Ohgaki H. Promoter hypermethylation of the RB1 gene in glioblastomas. Laboratory investigation. 2001;81(1):77-82. doi:10.1038/labinvest.3780213 
medRxiv preprint doi: https://doi.org/10.1101/2020.12.21.20248616; this version posted December 23, 2020. The copyright holder for this preprint (which was not certified by peer review) is the author/funder, who has granted medRxiv a license to display the preprint in perpetuity. All rights reserved. No reuse allowed without permission.

11.Lucio-Eterovic AK, Cortez MA, Valera ET, Motta FJ, Queiroz RG, Machado HR, Carlotti CG, Neder L, Scrideli CA, Tone LG. Differential expression of 12 histone deacetylase (HDAC) genes in astrocytomas and normal brain tissue: class II and IV are hypoexpressed in glioblastomas. BMC cancer. 2008;8(1):243. doi:10.1186/1471-2407-8-243

12.Kefas B, Godlewski J, Comeau L, Li Y, Abounader R, Hawkinson M, Lee J, Fine $\mathrm{H}$, Chiocca EA, Lawler $\mathrm{S}$ et al. microRNA-7 inhibits the epidermal growth factor receptor and the Akt pathway and is down-regulated in glioblastoma. Cancer research. 2008;68(10):3566-3572. doi:10.1158/00085472.CAN-07-6639

13.Liu Q, Xu X, Zhao M, Wei Z, Li X, Zhang X, Liu Z, Gong Y, Shao C. Berberine induces senescence of human glioblastoma cells by downregulating the EGFR-MEK-ERK signaling pathway. Molecular cancer therapeutics. 2015 1;14(2):355-363. doi:10.1158/1535-7163.MCT-14-0634

14. Yuan Y, Xue X, Guo RB, Sun XL, Hu G. Resveratrol enhances the antitumor effects of temozolomide in glioblastoma via ROS-dependent AMPK-TSC-mTOR signaling pathway. CNS neuroscience \& therapeutics. 2012;18(7):536-546. doi:10.1111/j.1755-5949.2012.00319.x

15.Zhang JQ, Hong B. miR520a-3p suppresses cell proliferation and metastasis by inhibiting the p65-NFKB pathway in glioblastoma. OncoTargets and therapy. 2019;12:6503-6513. doi:10.2147/OTT.S208889

16.Kugler W, Erdlenbruch B, Otten K, Jendrossek V, Eibl H, Lakomek M. MAP kinase pathways involved in glioblastoma response to erucylphosphocholine. International journal of oncology. 2004;25(6):17211727.

17. Kruthika BS, Jain R, Arivazhagan A, Bharath RD, Yasha TC, Kondaiah P, Santosh V. Transcriptome profiling reveals PDZ binding kinase as a novel biomarker in peritumoral brain zone of glioblastoma. Journal of neurooncology. 2019;141(2):315-325. doi:10.1007/s11060-018-03051-5

18.Dunning MJ, Smith ML, Ritchie ME, Tavaré S. beadarray: R classes and methods for Illumina bead-based data. Bioinformatics. 2007;23(16):21832184. doi:10.1093/bioinformatics/btm311

19.Ritchie ME, Phipson B, Wu D, Hu Y, Law CW, Shi W, Smyth GK. limma powers differential expression analyses for RNA-sequencing and microarray studies. Nucleic Acids Res. 2015;43(7):e47. doi:10.1093/nar/gkv007 
medRxiv preprint doi: https://doi.org/10.1101/2020.12.21.20248616; this version posted December 23, 2020. The copyright holder for this preprint (which was not certified by peer review) is the author/funder, who has granted medRxiv a license to display the preprint in perpetuity. All rights reserved. No reuse allowed without permission.

20.Chen J, Bardes EE, Aronow BJ, Jegga AG. ToppGene Suite for gene list enrichment analysis and candidate gene prioritization. Nucleic Acids Res. 2009;37(Web Server issue):W305-W311. doi:10.1093/nar/gkp427

21.Karp PD, Billington R, Caspi R, Fulcher CA, Latendresse M, Kothari A, Keseler IM, Krummenacker M, Midford PE, Ong Q et al. The BioCyc collection of microbial genomes and metabolic pathways. Brief Bioinform. 2019;20(4):1085-1093. doi:10.1093/bib/bbx085

22.Aoki-Kinoshita KF, Kanehisa M. Gene annotation and pathway mapping in KEGG. Methods Mol Biol. 2007;396:71-91. doi:10.1007/978-1-59745-5152_6

23.Schaefer CF, Anthony K, Krupa S, Buchoff J, Day M, Hannay T, Buetow KH. PID: the Pathway Interaction Database. Nucleic Acids Res. 2009;37(Database issue):D674-D679. doi:10.1093/nar/gkn653

24.Croft D, O'Kelly G, Wu G, Haw R, Gillespie M, Matthews L, Caudy M, Garapati P, Gopinath G, Jassal B et al. Reactome: a database of reactions, pathways and biological processes. Nucleic Acids Res. 2011;39(Database issue):D691-D697. doi:10.1093/nar/gkq1018

25.Liberzon A, Subramanian A, Pinchback R, Thorvaldsdóttir H, Tamayo P, Mesirov JP. Molecular signatures database (MSigDB) 3.0. Bioinformatics. 2011;27(12):1739-1740. doi:10.1093/bioinformatics/btr260

26.Dahlquist KD, Salomonis N, Vranizan K, Lawlor SC, Conklin BR. GenMAPP, a new tool for viewing and analyzing microarray data on biological pathways. Nat Genet. 2002 ;31(1):19-20. doi:10.1038/ng0502-19

27.Petri V, Jayaraman P, Tutaj M, Hayman GT, Smith JR, De Pons J, Laulederkind SJ, Lowry TF, Nigam R, Wang SJ et al. The pathway ontology - updates and applications. J Biomed Semantics. 2014;5(1):7. doi:10.1186/2041-1480-5-7

28. Mi H, Muruganujan A, Thomas PD. PANTHER in 2013: modeling the evolution of gene function, and other gene attributes, in the context of phylogenetic trees. Nucleic Acids Res. 2013;41(Database issue):D377D386. doi:10.1093/nar/gks1118

29.Jewison T, Su Y, Disfany FM, Liang Y, Knox C, Maciejewski A, Poelzer J, Huynh J, Zhou Y, Arndt D et al. SMPDB 2.0: big improvements to the Small Molecule Pathway Database. Nucleic Acids Res. 2014;42(Database issue):D478-D484. doi:10.1093/nar/gkt1067 
medRxiv preprint doi: https://doi.org/10.1101/2020.12.21.20248616; this version posted December 23, 2020. The copyright holder for this

30.Lewis SE. The Vision and Challenges of the Gene Ontology. Methods Mol Biol. 2017;1446:291-302. doi:10.1007/978-1-4939-3743-1_21

31.Kotlyar M, Pastrello C, Malik Z, Jurisica I. IID 2018 update: contextspecific physical protein-protein interactions in human, model organisms and domesticated species. Nucleic Acids Res. 2019;47(D1):D581-D589. doi:10.1093/nar/gky1037

32. Chatr-Aryamontri A, Oughtred R, Boucher L, Rust J, Chang C, Kolas NK, O'Donnell L, Oster S, Theesfeld C, Sellam A et al. The BioGRID interaction database: 2017 update. Nucleic Acids Res. 2017;45(D1):D369-D379. doi:10.1093/nar/gkw1102

33. Orchard S, Ammari M, Aranda B, Breuza L, Briganti L, Broackes-Carter F, Campbell NH, Chavali G, Chen C, del-Toro N et al. The MIntAct project-IntAct as a common curation platform for 11 molecular interaction databases. Nucleic Acids Res. 2014;42(Database issue):D358-D363. doi:10.1093/nar/gkt1115

34. Kotlyar M, Pastrello C, Sheahan N, Jurisica I. Integrated interactions database: tissue-specific view of the human and model organism interactomes. Nucleic Acids Res. 2016;44(D1):D536-D541. doi:10.1093/nar/gkv1115

35.Licata L, Briganti L, Peluso D, Perfetto L, Iannuccelli M, Galeota E, Sacco F, Palma A, Nardozza AP, Santonico E et al. MINT, the molecular interaction database: 2012 update. Nucleic Acids Res. 2012;40(Database issue):D857-D861. doi:10.1093/nar/gkr930

36. Breuer K, Foroushani AK, Laird MR, Chen C, Sribnaia A, Lo R, Winsor GL, Hancock RE, Brinkman FS, Lynn DJ. InnateDB: systems biology of innate immunity and beyond--recent updates and continuing curation. Nucleic Acids Res. 2013;41(Database issue):D1228-D1233. doi:10.1093/nar/gks1147

37. Salwinski L, Miller CS, Smith AJ, Pettit FK, Bowie JU, Eisenberg D. The Database of Interacting Proteins: 2004 update. Nucleic Acids Res. 2004;32(Database issue):D449-D451. doi:10.1093/nar/gkh086

38. Keshava Prasad TS, Goel R, Kandasamy K,Keerthikumar S, Kumar S, Mathivanan S, Telikicherla D, Raju R, Shafreen B, Venugopal A et al. Human Protein Reference Database--2009 update. Nucleic Acids Res. 2009;37(Database issue):D767-D772. doi:10.1093/nar/gkn892 
medRxiv preprint doi: https://doi.org/10.1101/2020.12.21.20248616; this version posted December 23, 2020. The copyright holder for this preprint (which was not certified by peer review) is the author/funder, who has granted medRxiv a license to display the preprint in perpetuity. All rights reserved. No reuse allowed without permission.

39.Bader GD, Betel D, Hogue CW. BIND: the Biomolecular Interaction Network Database. Nucleic Acids Res. 2003;31(1):248-250. doi:10.1093/nar/gkg056

40. Shannon P, Markiel A, Ozier O, Baliga NS, Wang JT, Ramage D, Amin N, Schwikowski B, Ideker T Cytoscape: a software environment for integrated models of biomolecular interaction networks. Genome Res 2003;13(11):2498-2504. doi:10.1101/gr.1239303

41.Zhao B, Wang J, Li M, Wu FX, Pan Y. Prediction of essential proteins based on overlapping essential modules. IEEE Trans Nanobioscience. 2014;13(4):415-424. doi:10.1109/TNB.2014.2337912

42.Asadzadeh-Aghdaee $\mathrm{H}$, Shahrokh S, Norouzinia M, Hosseini M, Keramatinia A, Jamalan M, Naghibzadeh B, Sadeghi A, Sherafat SJ, Zali MR.. Introduction of inflammatory bowel disease biomarkers panel using protein-protein interaction (PPI) network analysis. Gastroenterol Hepatol Bed Bench. 2016;9(Suppl1):S8-S13.

43.Rezaei-Tavirani M, Rezaei-Taviran S, Mansouri M, Rostami-Nejad M, Rezaei-Tavirani M. Protein-Protein Interaction Network Analysis for a Biomarker Panel Related to Human Esophageal Adenocarcinoma. Asian Pac J Cancer Prev. 2017;18(12):3357-3363. doi:10.22034/APJCP.2017.18.12.3357

44.Li G, Li M, Wang J, Li Y, Pan Y. United neighborhood closeness centrality and orthology for predicting essential proteins. IEEE/ACM Trans Comput Biol Bioinform. 2018;10.1109/TCBB.2018.2889978. doi:10.1109/TCBB.2018.2889978

45.Lei X, Wu S, Ge L, Zhang A. Clustering and overlapping modules detection in PPI network based on IBFO. Proteomics. 2013;13(2):278-290. doi:10.1002/pmic.201200309

46.Zaki N, Efimov D, Berengueres J. Protein complex detection using interaction reliability assessment and weighted clustering coefficient. BMC. Bioinformatics. 2013,14:163. doi:10.1186/1471-2105-14-163

47.Vlachos IS, Paraskevopoulou MD, Karagkouni D, Georgakilas G, Vergoulis T, Kanellos I, Anastasopoulos IL, Maniou S, Karathanou K, Kalfakakou D et al DIANA-TarBase v7.0: indexing more than half a million experimentally supported miRNA:mRNA interactions. Nucleic Acids Res. 2015;43(Database issue):D153-D159. doi:10.1093/nar/gku1215 
medRxiv preprint doi: https://doi.org/10.1101/2020.12.21.20248616; this version posted December 23, 2020. The copyright holder for this

48. Chou CH, Shrestha S, Yang CD, Chang NW, Lin YL, Liao KW, Huang WC, Sun TH, Tu SJ, Lee WH et al miRTarBase update 2018: a resource for experimentally validated microRNA-target interactions. Nucleic Acids Res. 2018;46(D1):D296-D302. doi:10.1093/nar/gkx1067

49. Zhou G, Soufan O, Ewald J, Hancock REW, Basu N, Xia J. NetworkAnalyst 3.0: a visual analytics platform for comprehensive gene expression profiling and meta-analysis. Nucleic Acids Res. 2019. doi:10.1093/nar/gkz240

50.Lachmann A, Xu H, Krishnan J, Berger SI, Mazloom AR, Ma'ayan A. ChEA: transcription factor regulation inferred from integrating genome-wide ChIP-X experiments. Bioinformatics. 2010;26(19):2438-2444. doi:10.1093/bioinformatics/btq466

51.Chandrashekar DS, Bashel B, Balasubramanya SAH, Creighton CJ, PonceRodriguez I, Chakravarthi BVSK, Varambally S. UALCAN: A Portal for Facilitating Tumor Subgroup Gene Expression and Survival Analyses. Neoplasia. 2017;19(8):649-658. doi:10.1016/j.neo.2017.05.002

52.Gao J, Aksoy BA, Dogrusoz U, Dresdner G, Gross B, Sumer SO, Sun Y, Jacobsen A, Sinha R, Larsson E et al. Integrative analysis of complex cancer genomics and clinical profiles using the cBioPortal. Sci Signal. 2013;6(269):pl1. doi:10.1126/scisignal.2004088

53. Uhlen M, Oksvold P, Fagerberg L, Lundberg E, Jonasson K, Forsberg M, Zwahlen M, Kampf C, Wester K, Hober S et al. Towards a knowledge-based Human Protein Atlas. Nat Biotechnol. 2010;28(12):1248-1250. doi:10.1038/nbt1210-1248

54.Robin X, Turck N, Hainard A, Tiberti N, Lisacek F, Sanchez JC, Müller M. pROC: an open-source package for $\mathrm{R}$ and $\mathrm{S}+$ to analyze and compare ROC curves. BMC Bioinformatics. 2011;12:77. doi:10.1186/1471-2105-12-77

55.Livak KJ, Schmittgen TD. Analysis of relative gene expression data using real-time quantitative PCR and the 2(-Delta Delta C(T)) Method. Methods. 2001;25(4):402-408. doi:10.1006/meth.2001.1262

56.Li T, Fan J, Wang B, Traugh N, Chen Q, Liu JS, Li B, Liu XS. TIMER: A Web Server for Comprehensive Analysis of Tumor-Infiltrating Immune Cells. Cancer Res. 2017;77(21):e108-e110. doi:10.1158/0008-5472.CAN17-0307 
medRxiv preprint doi: https://doi.org/10.1101/2020.12.21.20248616; this version posted December 23, 2020. The copyright holder for this preprint (which was not certified by peer review) is the author/funder, who has granted medRxiv a license to display the preprint in perpetuity. All rights reserved. No reuse allowed without permission.

57.Batash R, Asna N, Schaffer P, Francis N, Schaffer M. Glioblastoma Multiforme, Diagnosis and Treatment; Recent Literature Review. Curr Med Chem. 2017;24(27):3002-3009. doi:10.2174/0929867324666170516123206 58.Li Y, Dong X, Cai J, Yin S, Sun Y, Yang D, Jiang C. SERPINA3 induced by astroglia/microglia co-culture facilitates glioblastoma stem-like cell invasion. Oncol Lett. 2018;15(1):285-291. doi:10.3892/ol.2017.7275

59. Maugeri G, Grazia D’Amico A, Reitano R, Magro G, Cavallaro S, Salomone S, D'Agata V. PACAP and VIP Inhibit the Invasiveness of Glioblastoma Cells Exposed to Hypoxia through the Regulation of HIFs and EGFR Expression. Front Pharmacol. 2016;7:139. doi:10.3389/fphar.2016.00139

60.Tu Y, Xie P, Du X, Fan L, Bao Z, Sun G, Chao H, Li C, Zeng A, Pan M, et al. S100A11 functions as novel oncogene in glioblastoma via S100A11/ANXA2/NF- $\kappa$ B positive feedback loop. J Cell Mol Med. 2019;23(10):6907-6918. doi:10.1111/jcmm.14574

61.Barbieri F, Pattarozzi A, Gatti M, Porcile C, Bajetto A, Ferrari A, Culler $\mathrm{MD}$, Florio T. Somatostatin receptors 1, 2, and 5 cooperate in the somatostatin inhibition of C6 glioma cell proliferation in vitro via a phosphotyrosine phosphatase-eta-dependent inhibition of extracellularly regulated kinase-1/2. Endocrinology. 2008;149(9):4736-4746. doi:10.1210/en.2007-1762

62.Dave B, Gonzalez DD, Liu ZB, Li X, Wong H, Granados S, Ezzedine NE, Sieglaff DH, Ensor JE, Miller KD et al. Role of RPL39 in Metaplastic Breast Cancer. J Natl Cancer Inst. 2016;109(6):djw292. doi:10.1093/jnci/djw292

63. Wang J, Chen W, Wei W, Lou J. Oncogene TUBA1C promotes migration and proliferation in hepatocellular carcinoma and predicts a poor prognosis. Oncotarget. 2017;8(56):96215-96224. doi:10.18632/oncotarget.21894

64.Li H, Al-Japairai K, Tao Y, Xiang Z. RPN2 promotes colorectal cancer cell proliferation through modulating the glycosylation status of EGFR. Oncotarget. 2017;8(42):72633-72651. doi:10.18632/oncotarget.20005

65.Qiao F, Su X, Qiu X, Qian D, Peng X, Chen H, Zhao Z, Fan H. RASAL1 influences the proliferation and invasion of gastric cancer cells by regulating the RAS/ERK signaling pathway. Hum Cell. 2014;27(3):103-110. doi:10.1007/s13577-014-0090-2 
medRxiv preprint doi: https://doi.org/10.1101/2020.12.21.20248616; this version posted December 23, 2020. The copyright holder for this preprint (which was not certified by peer review) is the author/funder, who has granted medRxiv a license to display the preprint in perpetuity. All rights reserved. No reuse allowed without permission.

66.Clawson GA, Abraham T, Pan W, Tang X, Linton SS, McGovern CO, Loc WS, Smith JP, Butler PJ, Kester M, et al. A Cholecystokinin B ReceptorSpecific DNA Aptamer for Targeting Pancreatic Ductal Adenocarcinoma. Nucleic Acid Ther. 2017;27(1):23-35. doi:10.1089/nat.2016.0621

67.Tamir A, Jag U, Sarojini S, Schindewolf C, Tanaka T, Gharbaran R, Patel H, Sood A, Hu W, Patwa R, et al. Kallikrein family proteases KLK6 and KLK7 are potential early detection and diagnostic biomarkers for serous and papillary serous ovarian cancer subtypes. J Ovarian Res. 2014;7:109. doi:10.1186/s13048-014-0109-Z

68.Cheng Y, Dai C, Zhang J. SIRT3-SOD2-ROS pathway is involved in linalool-induced glioma cell apoptotic death. Acta Biochim Pol. 2017;64(2):343-350. doi:10.18388/abp.2016_1438

69. Yong WH, Shabihkhani M, Telesca D, Yang S, Tso JL, Menjivar JC, Wei B, Lucey GM, Mareninov S, Chen Z, et al. Ribosomal Proteins RPS11 and RPS20, Two Stress-Response Markers of Glioblastoma Stem Cells, Are Novel Predictors of Poor Prognosis in Glioblastoma Patients. PLoS One. 2015;10(10):e0141334. doi:10.1371/journal.pone.0141334

70.Tian N, Qi Y, Hu Y, Yin B, Yuan J, Qiang B, Peng X, Han W. RNAbinding Protein UNR Promotes Glioma Cell Migration and Regulates the Expression of Ribosomal Protein L9. Chin Med Sci J. 2018;33(3):143-151. doi:10.24920/11815

71.Wang J, Wang H, Li Z, Wu Q, Lathia JD, McLendon RE, Hjelmeland AB, Rich JN. c-Myc is required for maintenance of glioma cancer stem cells. PLoS One. 2008;3(11):e3769. doi:10.1371/journal.pone.0003769

72.Liu B, Liu J, Liao Y, Jin C, Zhang Z, Zhao J, Liu K, Huang H, Cao H, Cheng Q. Identification of SEC61G as a Novel Prognostic Marker for Predicting Survival and Response to Therapies in Patients with Glioblastoma. Med Sci Monit. 2019;25:3624-3635. doi:10.12659/MSM.916648

73.Kim YH, Thi-Anh-Thuy Tran HJ, Lee SI, Lee JJ, Jang WY, Moon KS, Kim IY, Jung S, Jung TY. Branched multipeptide immunotherapy for glioblastoma using human leukocyte antigen-A*0201-restricted cytotoxic Tlymphocyte epitopes from ERBB2, BIRC5 and CD99. Oncotarget. 2016;7(31):50535-50547. doi:10.18632/oncotarget.10495 
medRxiv preprint doi: https://doi.org/10.1101/2020.12.21.20248616; this version posted December 23, 2020. The copyright holder for this preprint (which was not certified by peer review) is the author/funder, who has granted medRxiv a license to display the preprint in perpetuity. All rights reserved. No reuse allowed without permission.

74.Wang J, Cheng P, Pavlyukov MS, Yu H, Zhang Z, Kim SH, Minata M, Mohyeldin A, Xie W, Chen D, et al. Targeting NEK2 attenuates glioblastoma growth and radioresistance by destabilizing histone methyltransferase EZH2. J Clin Invest. 2017;127(8):3075-3089. doi:10.1172/JCI89092

75.Li H, Jiang X, Yu Y, Huang W, Xing H, Agar NY, Yang HW, Yang B, Carroll RS, Johnson MD. KAP regulates ROCK2 and Cdk2 in an RNAactivated glioblastoma invasion pathway. Oncogene. 2015;34(11):14321441. doi:10.1038/onc.2014.49

76. Buczkowicz P, Zarghooni M, Bartels U, Morrison A, Misuraca KL, Chan T, Bouffet E, Huang A, Becher O, Hawkins C. Aurora kinase B is a potential therapeutic target in pediatric diffuse intrinsic pontine glioma. Brain Pathol. 2013;23(3):244-253. doi:10.1111/j.1750-3639.2012.00633.x

77.Kim W, Youn H, Lee S, Kim E, Kim D, Lee JS, Lee JM, Youn B. RNF138mediated ubiquitination of $\mathrm{rpS} 3$ is required for resistance of glioblastoma cells to radiation-induced apoptosis. Exp Mol Med. 2018;50(1):e434. doi:10.1038/emm.2017.247

78. Kuzontkoski PM, Mulligan-Kehoe MJ, Harris BT, Israel MA. Inhibitor of DNA binding-4 promotes angiogenesis and growth of glioblastoma multiforme by elevating matrix GLA levels. Oncogene. 2010;29(26):37933802. doi:10.1038/onc.2010.147

79.Ladha J, Sinha S, Bhat V, Donakonda S, Rao SM. Identification of genomic targets of transcription factor AEBP1 and its role in survival of glioma cells. Mol Cancer Res. 2012;10(8):1039-1051. doi:10.1158/1541-7786.MCR-110488

80.Liu J, Li W, Liu S, Zheng X, Shi L, Zhang W, Yang H. Knockdown of Collagen Triple Helix Repeat Containing 1 (CTHRC1) Inhibits EpithelialMesenchymal Transition and Cellular Migration in Glioblastoma Cells. Oncol Res. 2017;25(2):225-232. doi:10.3727/096504016X14732772150587

81.Sun S, Wang Y, Wu Y, Gao Y, Li Q, Abdulrahman AA, Liu XF, Ji GQ, Gao $\mathrm{J}$, Li L, et al. Identification of COL1A1 as an invasion-related gene in malignant astrocytoma. Int $\mathrm{J}$ Oncol. 2018;53(6):2542-2554. doi:10.3892/ijo.2018.4568

82.Gao YF, Mao XY, Zhu T, Mao CX, Liu ZX, Wang ZB, Li L, Li X, Yin JY, Zhang W, et al. COL3A1 and SNAP91: novel glioblastoma markers with 
medRxiv preprint doi: https://doi.org/10.1101/2020.12.21.20248616; this version posted December 23, 2020. The copyright holder for this

diagnostic and prognostic value. Oncotarget. 2016;7(43):70494-70503. doi:10.18632/oncotarget.12038

83. Miroshnikova YA, Mouw JK, Barnes JM, Pickup MW, Lakins JN, Kim Y, Lobo K, Persson AI, Reis GF, McKnight TR, et al. Tissue mechanics promote IDH1-dependent HIF1 $\alpha$-tenascin $\mathrm{C}$ feedback to regulate glioblastoma aggression. Nat Cell Biol. 2016;18(12):1336-1345. doi:10.1038/ncb3429

84.Zinn PO, Singh SK, Kotrotsou A, Hassan I, Thomas G, Luedi MM, Elakkad A, Elshafeey N, Idris T, Mosley J, et al. A Coclinical Radiogenomic Validation Study: Conserved Magnetic Resonance Radiomic Appearance of Periostin-Expressing Glioblastoma in Patients and Xenograft Models. Clin Cancer Res. 2018;24(24):6288-6299. doi:10.1158/1078-0432.CCR-17-3420 85.Gállego Pérez-Larraya J, Paris S, Idbaih A, Dehais C, Laigle-Donadey F, Navarro S, Capelle L, Mokhtari K, Marie Y, Sanson M, et al. Diagnostic and prognostic value of preoperative combined GFAP, IGFBP-2, and YKL-40 plasma levels in patients with glioblastoma. Cancer. 2014;120(24):39723980. doi:10.1002/cncr.28949

86. Abdolhoseinpour H, Mehrabi F, Shahraki K, Khoshnood RJ, Masoumi B, Yahaghi E, Goudarzi PK.Investigation of serum levels and tissue expression of two genes IGFBP-2 and IGFBP-3 act as potential biomarker for predicting the progression and survival in patients with glioblastoma multiforme. J Neurol Sci. 2016;366:202-206. doi:10.1016/j.jns.2016.05.018

87. Moreno MJ, Ball M, Andrade MF, McDermid A, Stanimirovic DB. Insulinlike growth factor binding protein-4 (IGFBP-4) is a novel anti-angiogenic and anti-tumorigenic mediator secreted by dibutyryl cyclic AMP (dBcAMP)-differentiated glioblastoma cells. Glia. 2006;53(8):845-857. doi:10.1002/glia.20345

88. Tang H, Zhao J, Zhang L, Zhao J, Zhuang Y, Liang P. SRPX2 Enhances the Epithelial-Mesenchymal Transition and Temozolomide Resistance in Glioblastoma Cells. Cell Mol Neurobiol. 2016;36(7):1067-1076. doi:10.1007/s10571-015-0300-9

89.Chen Q, Lu G, Cai Y, Li Y, Xu R, Ke Y, Zhang S. MiR-124-5p inhibits the growth of high-grade gliomas through posttranscriptional regulation of LAMB1. Neuro Oncol. 2014;16(5):637-651. doi:10.1093/neuonc/not300 
medRxiv preprint doi: https://doi.org/10.1101/2020.12.21.20248616; this version posted December 23, 2020. The copyright holder for this preprint (which was not certified by peer review) is the author/funder, who has granted medRxiv a license to display the preprint in perpetuity. All rights reserved. No reuse allowed without permission.

90.Wang J, Zuo J, Wahafu A, Yu H, Xie W, Zhang H, Wang M. ESM1dependent mesenchymal transition enhances radioresistance of glioblastoma via transcriptional regulation of $\mathrm{NF \kappa B}$ [published online ahead of print, 2019 Oct 24]. Biochem Biophys Res Commun. 2019;S0006291X(19)32028-5. doi:10.1016/j.bbrc.2019.10.126

91.Lin B, Madan A, Yoon JG, Fang X, Yan X, Kim TK, Hwang D, Hood L, Foltz G. Massively parallel signature sequencing and bioinformatics analysis identifies up-regulation of TGFBI and SOX4 in human glioblastoma. PLoS One. 2010;5(4):e10210. doi:10.1371/journal.pone.0010210

92.Feng L, Ma J, Ji H, Liu Y, Hu W. miR-330-5p suppresses glioblastoma cell proliferation and invasiveness through targeting ITGA5. Biosci Rep. 2017;37(3):BSR20170019. doi:10.1042/BSR20170019

93. She X, Yu Z, Cui Y, Lei Q, Wang Z, Xu G, Xiang J, Wu M, Li G. miR-128 and miR-149 enhance the chemosensitivity of temozolomide by Rap1Bmediated cytoskeletal remodeling in glioblastoma. Oncol Rep. 2014;32(3):957-964. doi:10.3892/or.2014.3318

94.Quann K, Gonzales DM, Mercier I, Wang C, Sotgia F, Pestell RG, Lisanti MP, Jasmin JF. Caveolin-1 is a negative regulator of tumor growth in glioblastoma and modulates chemosensitivity to temozolomide. Cell Cycle. 2013;12(10):1510-1520. doi:10.4161/cc.24497

95. Ghosh D, Ulasov IV, Chen L, Harkins LE, Wallenborg K, Hothi P, Rostad S, Hood L, Cobbs CS. TGF $\beta$-Responsive HMOX1 Expression Is Associated with Stemness and Invasion in Glioblastoma Multiforme. Stem Cells. 2016;34(9):2276-2289. doi:10.1002/stem.2411

96.Han S, Feng S, Yuan G, Dong T, Gao D, Liang G, Wei X. Lysyl oxidase genetic variants and the prognosis of glioma. APMIS. 2014;122(3):200-205. doi:10.1111/apm.12133

97.Chen Z, Hu T, Zhu S, Mukaisho K, El-Rifai W, Peng DF. Glutathione peroxidase 7 suppresses cancer cell growth and is hypermethylated in gastric cancer. Oncotarget. 2017;8(33):54345-54356. doi:10.18632/oncotarget.17527

98.Liu JJ, Huang BH, Zhang J, Carson DD, Hooi SC. Repression of HIP/RPL29 expression induces differentiation in colon cancer cells. J Cell Physiol. 2006;207(2):287-292. doi:10.1002/jcp.20589 
medRxiv preprint doi: https://doi.org/10.1101/2020.12.21.20248616; this version posted December 23, 2020. The copyright holder for this preprint (which was not certified by peer review) is the author/funder, who has granted medRxiv a license to display the preprint in perpetuity. All rights reserved. No reuse allowed without permission.

99.He Z, Xu Q, Wang X, Wang J, Mu X, Cai Y, Qian Y, Shao W, Shao Z. RPLP1 promotes tumor metastasis and is associated with a poor prognosis in triple-negative breast cancer patients. Cancer Cell Int. 2018;18:170. doi:10.1186/s12935-018-0658-0

100. Wang M, Hu Y, Stearns ME. RPS2: a novel therapeutic target in prostate cancer. J Exp Clin Cancer Res. 2009;28(1):6. doi:10.1186/17569966-28-6

101. Slizhikova DK, Vinogradova TV, Sverdlov ED. The NOLA2 and RPS3A genes as highly informative markers of human squamous cell carcinoma of lung. Russian Journal of Bioorganic Chemistry. 2005;31(2):178-182. doi:10.1007/s11171-005-0024-6

102. Guo X, Shi Y, Gou Y, Li J, Han S, Zhang Y, Huo J, Ning X, Sun L, Chen $\mathrm{Y}$, et al. Human ribosomal protein S13 promotes gastric cancer growth through down-regulating p27(Kip1). J Cell Mol Med. 2011;15(2):296-306. doi:10.1111/j.1582-4934.2009.00969.x

103. Liu C, He X, Liu X, Yu J, Zhang M, Yu F, Wang Y. RPS15A promotes gastric cancer progression via activation of the Akt/IKK- $\beta / \mathrm{NF}-\kappa \mathrm{B}$ signalling pathway. J Cell Mol Med. 2019;23(3):2207-2218. doi:10.1111/jcmm.14141

104. Zhu Y, Lin H, Li Z, Wang M, Luo J. Modulation of expression of ribosomal protein L7a (rpL7a) by ethanol in human breast cancer cells. Breast Cancer Res Treat. 2001;69(1):29-38. doi:10.1023/a:1012293507534

105. Qiu JJ, Guo JJ, Lv TJ, Jin HY, Ding JX, Feng WW, Zhang Y, Hua KQ. Prognostic value of centromere protein-A expression in patients with epithelial ovarian cancer. Tumour Biol. 2013;34(5):2971-2975. doi:10.1007/s13277-013-0860-6

106. Zhuo YJ, Xi M, Wan YP, Hua W, Liu YL, Wan S, Zhou YL, Luo $\mathrm{HW}, \mathrm{Wu}$ SL, Zhong WD, et al. Enhanced expression of centromere protein F predicts clinical progression and prognosis in patients with prostate cancer. Int J Mol Med. 2015;35(4):966-972. doi:10.3892/ijmm.2015.2086

107. Furic L, Rong L, Larsson O, Koumakpayi IH, Yoshida K, Brueschke A, Petroulakis E, Robichaud N, Pollak M, Gaboury LA, et al. eIF4E phosphorylation promotes tumorigenesis and is associated with prostate cancer progression. Proc Natl Acad Sci U S A. 2010;107(32):14134-14139. doi:10.1073/pnas.1005320107 
medRxiv preprint doi: https://doi.org/10.1101/2020.12.21.20248616; this version posted December 23, 2020. The copyright holder for this preprint (which was not certified by peer review) is the author/funder, who has granted medRxiv a license to display the preprint in perpetuity. All rights reserved. No reuse allowed without permission.

108. Wang GH, Yao L, Xu HW, Tang WT, Fu JH, Hu XF, Cui L, Xu XM.Identification of MXRA5 as a novel biomarker in colorectal cancer. Oncol Lett. 2013;5(2):544-548. doi:10.3892/ol.2012.1038

109. Coulson-Thomas VJ, Coulson-Thomas YM, Gesteira TF, de Paula CA, Carneiro CR, Ortiz V, Toma L, Kao WW, Nader HB.Lumican expression, localization and antitumor activity in prostate cancer. Exp Cell Res. 2013;319(7):967-981. doi:10.1016/j.yexcr.2013.01.023

110. Chen D, Zhang R, Shen W, Fu H, Liu S, Sun K, Sun X. RPS12specific shRNA inhibits the proliferation, migration of BGC823 gastric cancer cells with S100A4 as a downstream effector. Int $\mathbf{J}$ Oncol. 2013;42(5):1763-1769. doi:10.3892/ijo.2013.1872

111. Wu Q, Gou Y, Wang Q, Jin H, Cui L, Zhang Y, He L, Wang J, Nie Y, Shi Y, et al. Downregulation of RPL6 by siRNA inhibits proliferation and cell cycle progression of human gastric cancer cell lines. PLoS One. 2011;6(10):e26401. doi:10.1371/journal.pone.0026401

112. Yang ZX, Zhang B, Wei J, Jiang GQ, Wu YL, Leng BJ, Xing CG. MiR-539 inhibits proliferation and migration of triple-negative breast cancer cells by down-regulating LAMA4 expression. Cancer Cell Int. 2018;18:16. doi:10.1186/s12935-018-0512-4

113. Shi Q, Wang W, Jia Z, Chen P, Ma K, Zhou C. ISL1, a novel regulator of CCNB1, CCNB2 and c-MYC genes, promotes gastric cancer cell proliferation and tumor growth. Oncotarget. 2016;7(24):36489-36500. doi:10.18632/oncotarget.9269

114. Gao CL, Wang GW, Yang GQ, Yang H, Zhuang L. Karyopherin subunit- $\alpha 2$ expression accelerates cell cycle progression by upregulating CCNB2 and CDK1 in hepatocellular carcinoma. Oncol Lett. 2018;15(3):2815-2820. doi:10.3892/ol.2017.7691

115. Thorenoor N, Faltejskova-Vychytilova P, Hombach S, Mlcochova J, Kretz M, Svoboda M, Slaby O. Long non-coding RNA ZFAS1 interacts with CDK1 and is involved in p53-dependent cell cycle control and apoptosis in colorectal cancer. Oncotarget. 2016;7(1):622-637. doi:10.18632/oncotarget.5807

116. Goh SH, Hong SH, Lee BC, Ju MH, Jeong JS, Cho YR, Kim IH, Lee YS. eIF3m expression influences the regulation of tumorigenesis-related 
medRxiv preprint doi: https://doi.org/10.1101/2020.12.21.20248616; this version posted December 23, 2020. The copyright holder for this

genes in human colon cancer. Oncogene. 2011;30(4):398-409. doi:10.1038/onc.2010.422

117. Shriver SP, Shriver MD, Tirpak DL, Bloch LM, Hunt JD, Ferrell RE, Siegfried JM. Trinucleotide repeat length variation in the human ribosomal protein L14 gene (RPL14): localization to 3p21.3 and loss of heterozygosity in lung and oral cancers. Mutat Res. 1998;406(1):9-23. doi:10.1016/s13835726(98)00006-5

118. Lou J, Gong J, Ke J, Tian J, Zhang Y, Li J, Yang Y, Zhu Y, Gong Y, Li L, et al. A functional polymorphism located at transcription factor binding sites, rs6695837 near LAMC1 gene, confers risk of colorectal cancer in Chinese populations. Carcinogenesis. 2017;38(2):177-183. doi:10.1093/carcin/bgw204

119. Yan TT, Fu XL, Li J, Bian YN, Liu DJ, Hua R, Ren LL, Li CT, Sun YW, Chen HY, et al. Downregulation of RPL15 may predict poor survival and associate with tumor progression in pancreatic ductal adenocarcinoma. Oncotarget. 2015;6(35):37028-37042. doi:10.18632/oncotarget.5939

120. Li X, Li J, Li F. P21 activated kinase 4 binds translation elongation factor eEF1A1 to promote gastric cancer cell migration and invasion. Oncol Rep. 2017;37(5):2857-2864. doi:10.3892/or.2017.5543

121. Liu CL, Pan HW, Torng PL, Fan MH, Mao TL. SRPX and HMCN1 regulate cancer-associated fibroblasts to promote the invasiveness of ovarian carcinoma. Oncol Rep. 2019;42(6):2706-2715. doi:10.3892/or.2019.7379

122. Ao R, Guan L, Wang Y, Wang JN. Silencing of COL1A2, COL6A3, and THBS2 inhibits gastric cancer cell proliferation, migration, and invasion while promoting apoptosis through the PI3k-Akt signaling pathway. J Cell Biochem. 2018;119(6):4420-4434. doi:10.1002/jcb.26524

123. Huang $\mathrm{R}, \mathrm{Gu} \mathrm{W}$, Sun $\mathrm{B}$, Gao L. Identification of COL4A1 as a potential gene conferring trastuzumab resistance in gastric cancer based on bioinformatics analysis. Mol Med Rep. 2018;17(5):6387-6396. doi:10.3892/mmr.2018.8664

124. Liu W, Wei H, Gao Z, Chen G, Liu Y, Gao X, Bai G, He S, Liu T, Xu $\mathrm{W}$, et al. COL5A1 may contribute the metastasis of lung adenocarcinoma. Gene. 2018;665:57-66. doi:10.1016/j.gene.2018.04.066 
medRxiv preprint doi: https://doi.org/10.1101/2020.12.21.20248616; this version posted December 23, 2020. The copyright holder for this

125. Zeng XT, Liu XP, Liu TZ, Wang XH. The clinical significance of COL5A2 in patients with bladder cancer: A retrospective analysis of bladder cancer gene expression data. Medicine (Baltimore). 2018;97(10):e0091. doi:10.1097/MD.0000000000010091

126. Shang J, Wang F, Chen P, Wang X, Ding F, Liu S, Zhao Q. Coexpression Network Analysis Identified COL8A1 Is Associated with the Progression and Prognosis in Human Colon Adenocarcinoma. Dig Dis Sci. 2018;63(5):1219-1228. doi:10.1007/s10620-018-4996-5

127. Andreuzzi E, Capuano A, Pellicani R, Poletto E, Doliana R, Maiero S, Fornasarig M, Magris R, Colombatti A, Cannizzaro R, et al. Loss of Multimerin-2 and EMILIN-2 Expression in Gastric Cancer Associate with Altered Angiogenesis. Int J Mol Sci. 2018;19(12):3983. doi:10.3390/ijms19123983

128. Yu C, Yu J, Yao X, Wu WK, Lu Y, Tang S, Li X, Bao L, Li X, Hou $\mathrm{Y}$, et al. Discovery of biclonal origin and a novel oncogene SLC12A5 in colon cancer by single-cell sequencing. Cell Res. 2014;24(6):701-712. doi:10.1038/cr.2014.43

129. Freier K, Sticht C, Hofele C, Flechtenmacher C, Stange D, Puccio L, Toedt G, Radlwimmer B, Lichter P, Joos S. Recurrent coamplification of cytoskeleton-associated genes EMS1 and SHANK2 with CCND1 in oral squamous cell carcinoma. Genes Chromosomes Cancer. 2006;45(2):118125. doi: $10.1002 / \mathrm{gcc} .20270$

130. Wu XY, Yu XY. Overexpression of KCNJ4 correlates with cancer progression and unfavorable prognosis in lung adenocarcinoma. J Biochem Mol Toxicol. 2019;33(4):e22270. doi:10.1002/jbt.22270

131. Natrajan R, Little SE, Reis-Filho JS, Hing L, Messahel B, Grundy PE, Dome JS, Schneider T, Vujanic GM, Pritchard-Jones K, et al. Amplification and overexpression of CACNA1E correlates with relapse in favorable histology Wilms' tumors. Clin Cancer Res. 2006;12(24):7284-7293. doi:10.1158/1078-0432.CCR-06-1567

132. Maolakuerban N, Azhati B, Tusong H, Abula A, Yasheng A, Xireyazidan A. MiR-200c-3p inhibits cell migration and invasion of clear cell renal cell carcinoma via regulating SLC6A1. Cancer Biol Ther. 2018;19(4):282-291. doi:10.1080/15384047.2017.1394551 
medRxiv preprint doi: https://doi.org/10.1101/2020.12.21.20248616; this version posted December 23, 2020. The copyright holder for this preprint (which was not certified by peer review) is the author/funder, who has granted medRxiv a license to display the preprint in perpetuity. All rights reserved. No reuse allowed without permission.

133. Longqiu Y, Pengcheng L, Xuejie F, Peng Z. A miRNAs panel promotes the proliferation and invasion of colorectal cancer cells by targeting GABBR1. Cancer Med. 2016;5(8):2022-2031. doi:10.1002/cam4.760

134. Kimura R, Kasamatsu A, Koyama T, Fukumoto C, Kouzu Y, Higo M, Endo-Sakamoto Y, Ogawara K, Shiiba M, Tanzawa H et al. Kimura R, Kasamatsu A, Koyama T, et al. Glutamate acid decarboxylase 1 promotes metastasis of human oral cancer by $\beta$-catenin translocation and MMP7 activation. BMC Cancer. 2013;13:555. doi:10.1186/1471-2407-13-555

135. Liu Z, Wang J, Li Y, Fan J, Chen L, Xu R. MicroRNA-153 regulates glutamine metabolism in glioblastoma through targeting glutaminase. Tumour Biol. 2017;39(2):1010428317691429. doi:10.1177/1010428317691429

136. Wang ZY, Xiong J, Zhang SS, Wang JJ, Gong ZJ, Dai MH. UpRegulation of microRNA-183 Promotes Cell Proliferation and Invasion in Glioma By Directly Targeting NEFL. Cell Mol Neurobiol. 2016;36(8):1303-1310. doi:10.1007/s10571-016-0328-5

137. Yucebas M, Susluer SY, Caglar HO, Balci T, Sigva ZO, Akalin T, Oktar N, Dalbasti T, Avci CB, Gunduz C. Expression profiling of RE1silencing transcription factor (REST), REST corepressor 1 (RCOR1), and Synapsin 1 (SYN1) genes in human gliomas. J BUON. 2016;21(4):964-972.

138. Lin B, Lee H, Yoon JG, Madan A, Wayner E, Tonning S, Hothi P, Schroeder B, Ulasov I, Foltz G, et al. Global analysis of H3K4me3 and H3K27me3 profiles in glioblastoma stem cells and identification of SLC17A7 as a bivalent tumor suppressor gene. Oncotarget. 2015;6(7):53695381. doi:10.18632/oncotarget.3030

139. Xiao B, Li J, Fan Y, Ye M, Lv S, Xu B, Chai Y, Zhou Z, Wu M, Zhu X. Downregulation of SYT7 inhibits glioblastoma growth by promoting cellular apoptosis. Mol Med Rep. 2017;16(6):9017-9022. doi:10.3892/mmr.2017.7723

140. Han X, Wang X, Li H, Zhang H. Mechanism of microRNA-431-5pEPB41L1 interaction in glioblastoma multiforme cells. Arch Med Sci. 2019;15(6):1555-1564. doi:10.5114/aoms.2019.88274 
medRxiv preprint doi: https://doi.org/10.1101/2020.12.21.20248616; this version posted December 23, 2020. The copyright holder for this preprint (which was not certified by peer review) is the author/funder, who has granted medRxiv a license to display the preprint in perpetuity. All rights reserved. No reuse allowed without permission.

141. Jhaveri A, Deshpande P, Pattni B, Torchilin V. Transferrin-targeted, resveratrol-loaded liposomes for the treatment of glioblastoma. J Control Release. 2018;277:89-101. doi:10.1016/j.jconrel.2018.03.006

142. Hauptman N, Jevšinek Skok D, Spasovska E, Boštjančič E, Glavač D. Genes CEP55, FOXD3, FOXF2, GNAO1, GRIA4, and KCNA5 as potential diagnostic biomarkers in colorectal cancer. BMC Med Genomics. 2019;12(1):54. doi:10.1186/s12920-019-0501-z

143. Basile MS, Fagone P, Mangano K, Mammana S, Magro G, Salvatorelli L, Li Destri G, La Greca G, Nicoletti F, Puleo S, et al. KCNMA1 Expression is Downregulated in Colorectal Cancer via Epigenetic Mechanisms. Cancers (Basel). 2019;11(2):245. doi:10.3390/cancers11020245

144. Kim JH, Kim TW, Kim SJ. Downregulation of ARFGEF1 and CAMK2B by promoter hypermethylation in breast cancer cells. BMB Rep. 2011;44(8):523-528. doi:10.5483/bmbrep.2011.44.8.523

145. Velmurugan BK, Yeh KT, Hsieh MJ, Yeh CM, Lin CC, Kao CY, Huang LR, Lin SH. UNC13C Suppress Tumor Progression via Inhibiting EMT Pathway and Improves Survival in Oral Squamous Cell Carcinoma. Front Oncol. 2019;9:728. doi:10.3389/fonc.2019.00728

146. Wang CT, Chen TM, Mei CT, Chang CF, Liu LL, Chiu KH, Wu TM, Lan YC, Liu WS, Chen YH, et al. The Functional Haplotypes of CHRM3 Modulate mRNA Expression and Associate with Bladder Cancer among a Chinese Han Population in Kaohsiung City. Biomed Res Int. 2016;2016:4052846. doi:10.1155/2016/4052846

147. Zhi T, Jiang K, Xu X, Yu T, Wu W, Nie E, Zhou X, Jin X, Zhang J, Wang Y, et al. MicroRNA-520d-5p inhibits human glioma cell proliferation and induces cell cycle arrest by directly targeting PTTG1. Am J Transl Res. 2017;9(11):4872-4887.

148. Hong B, Muili K, Bolyard C, Russell L, Lee TJ, BanasavadiSiddegowda Y, Yoo JY, Yan Y, Ballester LY, Bockhorst KH, et al. Suppression of HMGB1 Released in the Glioblastoma Tumor Microenvironment Reduces Tumoral Edema. Mol Ther Oncolytics. 2018;12:93-102. doi:10.1016/j.omto.2018.11.005

149. Tang C, Yang Z, Chen D, Xie Q, Peng T, Wu J, Qi S. Downregulation of miR-130a promotes cell growth and epithelial to mesenchymal transition 
medRxiv preprint doi: https://doi.org/10.1101/2020.12.21.20248616; this version posted December 23, 2020. The copyright holder for this

by activating HMGB2 in glioma. Int J Biochem Cell Biol. 2017;93:25-31. doi:10.1016/j.biocel.2017.08.010

150. Li J, Zhou Y, Wang H, Gao Y, Li L, Hwang SH, Ji X, Hammock BD. COX-2/sEH dual inhibitor PTUPB suppresses glioblastoma growth by targeting epidermal growth factor receptor and hyaluronan mediated motility receptor. Oncotarget. 2017;8(50):87353-87363. doi:10.18632/oncotarget.20928

151. Areshkov PA, Kavsan VM. Chitinase 3-like protein 2 (CHI3L2, YKL39) activates phosphorylation of extracellular signal-regulated kinases ERK1/ERK2 in human embryonic kidney (HEK293) and human glioblastoma (U87 MG) cells. Tsitol Genet. 2010;44(1):3-9.

152. Gong J, Zhu S, Zhang Y, Wang J. Interplay of VEGFa and MMP2 regulates invasion of glioblastoma. Tumour Biol. 2014;35(12):1187911885. doi:10.1007/s13277-014-2438-3

153. Balzeau J, Peterson A, Eyer J. The vimentin-tubulin binding site peptide (Vim-TBS.58-81) crosses the plasma membrane and enters the nuclei of human glioma cells. Int J Pharm. 2012;423(1):77-83. doi:10.1016/j.ijpharm.2011.04.067

154. Suvasini R, Shruti B, Thota B, Shinde SV, Friedmann-Morvinski D, Nawaz Z, Prasanna KV, Thennarasu K, Hegde AS, Arivazhagan A, et al. Insulin growth factor-2 binding protein 3 (IGF2BP3) is a glioblastomaspecific marker that activates phosphatidylinositol 3-kinase/mitogenactivated protein kinase (PI3K/MAPK) pathways by modulating IGF-2. J Biol Chem. 2011;286(29):25882-25890. doi:10.1074/jbc.M110.178012

155. Boukhari A, Alhosin M, Bronner C, Sagini K, Truchot C, Sick E, Schini-Kerth VB, Andre P, Mely Y, Mousli M, et al. CD47 activationinduced UHRF1 over-expression is associated with silencing of tumor suppressor gene p16INK4A in glioblastoma cells. Anticancer Res. 2015;35(1):149-157.

156. Yang W, Wang L, Roehn G, Pearlstein RD, Ali $\square$ Osman F, Pan H, Goldbrunner R, Krantz M, Harms C, Paschen W. Small ubiquitin-like modifier 1-3 conjugation is activated in human astrocytic brain tumors and is required for glioblastoma cell survival. Cancer Sci. 2013;104(1):70-77. doi:10.1111/cas.12047 
medRxiv preprint doi: https://doi.org/10.1101/2020.12.21.20248616; this version posted December 23, 2020. The copyright holder for this

157. Kruthika BS, Jain R, Arivazhagan A, Bharath RD, Yasha TC, Kondaiah P, Santosh V. Transcriptome profiling reveals PDZ binding kinase as a novel biomarker in peritumoral brain zone of glioblastoma. J Neurooncol. 2019;141(2):315-325. doi:10.1007/s11060-018-03051-5

158. Qiao W, Guo B, Zhou H, Xu W, Chen Y, Liang Y, Dong B. miR-124 suppresses glioblastoma growth and potentiates chemosensitivity by inhibiting AURKA. Biochem Biophys Res Commun. 2017;486(1):43-48. doi:10.1016/j.bbrc.2017.02.120

159. Yan Y, Xu Z, Chen X, Wang X, Zeng S, Zhao Z, Qian L, Li Z, Wei J, Huo L, et al. Novel Function of lncRNA ADAMTS9-AS2 in Promoting Temozolomide Resistance in Glioblastoma via Upregulating the FUS/MDM2 Ubiquitination Axis. Front Cell Dev Biol. 2019;7:217. doi:10.3389/fcell.2019.00217

160. Guo L, Ding Z, Huang N, Huang Z, Zhang N, Xia Z. Forkhead Box M1 positively regulates UBE2C and protects glioma cells from autophagic death. Cell Cycle. 2017;16(18):1705-1718. doi:10.1080/15384101.2017.1356507

161. Bassett EA, Palanichamy K, Pearson M, McElroy JP, Haque SJ, Bell

$\mathrm{EH}$, Chakravarti A. Calpastatin phosphorylation regulates radiation-induced calpain activity in glioblastoma. Oncotarget. 2018;9(18):14597-14607. doi:10.18632/oncotarget.24523

162. Panner A, Crane CA, Weng C, Feletti A, Fang S, Parsa AT, Pieper RO. Ubiquitin-specific protease 8 links the PTEN-Akt-AIP4 pathway to the control of FLIPS stability and TRAIL sensitivity in glioblastoma multiforme. Cancer Res. 2010;70(12):5046-5053. doi:10.1158/00085472.CAN-09-3979

163. Aaberg-Jessen C, Sørensen MD, Matos AL, Moreira JM, Brünner N, Knudsen A, Kristensen BW. Co-expression of TIMP-1 and its cell surface binding partner CD63 in glioblastomas. BMC Cancer. 2018;18(1):270. doi:10.1186/s12885-018-4179-y

164. Solga R, Behrens J, Ziemann A, Riou A, Berwanger C, Becker L, Garrett L, de Angelis MH, Fischer L, Coras R, et al. CRN2 binds to TIMP4 and MMP14 and promotes perivascular invasion of glioblastoma cells. Eur J Cell Biol. 2019;98(5-8):151046. doi:10.1016/j.ejcb.2019.151046 
medRxiv preprint doi: https://doi.org/10.1101/2020.12.21.20248616; this version posted December 23, 2020. The copyright holder for this preprint (which was not certified by peer review) is the author/funder, who has granted medRxiv a license to display the preprint in perpetuity. All rights reserved. No reuse allowed without permission.

165. Brown DV, Filiz G, Daniel PM, Hollande F, Dworkin S, Amiridis S, Kountouri N, Ng W, Morokoff AP, Mantamadiotis T. Expression of CD133 and CD44 in glioblastoma stem cells correlates with cell proliferation, phenotype stability and intra-tumor heterogeneity. PLoS One. 2017;12(2):e0172791. doi:10.1371/journal.pone.0172791

166. Karkavelas G, Mavropoulou S, Fountzilas G, Christoforidou V, Karavelis A, Foroglou G, Papadimitriou C. Correlation of proliferating cell nuclear antigen assessment, histologic parameters and age with survival in patients with glioblastoma multiforme. Anticancer Res. 1995;15(2):531536.

167. Qiu X, He X, Huang Q, Liu X, Sun G, Guo J, Yuan D, Yang L, Ban $\mathrm{N}$, Fan S, et al. Overexpression of CCT8 and its significance for tumor cell proliferation, migration and invasion in glioma. Pathol Res Pract. 2015;211(10):717-725. doi:10.1016/j.prp.2015.04.012

168. Wang Y, Wong CW, Yan M, Li L, Liu T, Or PM, Tsui SK, Waye MM, Chan AM. Differential regulation of the pro-inflammatory biomarker, YKL-40/CHI3L1, by PTEN/Phosphoinositide 3-kinase and JAK2/STAT3 pathways in glioblastoma. Cancer Lett. 2018;429:54-65. doi:10.1016/j.canlet.2018.04.040

169. Cheng SX, Tu Y, Zhang S. FoxM1 promotes glioma cells progression by up-regulating Anxa1 expression. PLoS One. 2013;8(8):e72376. doi:10.1371/journal.pone.0072376

170. Huang H, Han Y, Zhang C, Wu J, Feng J, Qu L, Shou C. HNRNPC as a candidate biomarker for chemoresistance in gastric cancer. Tumour Biol. 2016;37(3):3527-3534. doi:10.1007/s13277-015-4144-1

171. Wu FH, Yuan Y, Li D, Liao SJ, Yan B, Wei JJ, Zhou YH, Zhu JH, Zhang GM, Feng ZH. Extracellular HSPA1A promotes the growth of hepatocarcinoma by augmenting tumor cell proliferation and apoptosisresistance. Cancer Lett. 2012;317(2):157-164. doi:10.1016/j.canlet.2011.11.020

172. Wang J, Chen W, Wei W, Lou J. Oncogene TUBA1C promotes migration and proliferation in hepatocellular carcinoma and predicts a poor prognosis. Oncotarget. 2017;8(56):96215-96224. doi:10.18632/oncotarget.21894 
medRxiv preprint doi: https://doi.org/10.1101/2020.12.21.20248616; this version posted December 23, 2020. The copyright holder for this preprint (which was not certified by peer review) is the author/funder, who has granted medRxiv a license to display the preprint in perpetuity. All rights reserved. No reuse allowed without permission.

173. Shi YX, Zhu T, Zou T, Zhuo W, Chen YX, Huang MS, Zheng W, Wang CJ, Li X, Mao XY et al. Prognostic and predictive values of CDK1 and MAD2L1 in lung adenocarcinoma. Oncotarget. 2016;7(51):8523585243. doi:10.18632/oncotarget.13252

174. Hoffmann C, Mao X, Brown-Clay J, Moreau F, Al Absi A, Wurzer H, Sousa B, Schmitt F, Berchem G, Janji B et al. Hypoxia promotes breast cancer cell invasion through HIF-1 $\alpha$-mediated up-regulation of the invadopodial actin bundling protein CSRP2. Sci Rep. 2018;8(1):10191. doi:10.1038/s41598-018-28637-x

175. Shibamoto M, Hirata H, Eguchi H, Sawada G, Sakai N, Kajiyama Y, Mimori K. The loss of CASP4 expression is associated with poor prognosis in esophageal squamous cell carcinoma. Oncol Lett. 2017;13(3):1761-1766. doi:10.3892/ol.2017.5646

176. Liao H, Duka T, Teng FY, Sun L, Bu WY, Ahmed S, Tang BL, Xiao ZC. Nogo-66 and myelin-associated glycoprotein (MAG) inhibit the adhesion and migration of Nogo-66 receptor expressing human glioma cells. J Neurochem. 2004;90(5):1156-1162. doi:10.1111/j.14714159.2004.02573.x

177. Vila-Carriles WH, Zhou ZH, Bubien JK, Fuller CM, Benos DJ. Participation of the chaperone $\mathrm{Hsc70}$ in the trafficking and functional expression of ASIC2 in glioma cells. J Biol Chem. 2007;282(47):3438134391. doi:10.1074/jbc.M705354200

178. Devireddy LR, Kumar KU, Pater MM, Pater A. Evidence for a mechanism of demyelination by human JC virus: negative transcriptional regulation of RNA and protein levels from myelin basic protein gene by large tumor antigen in human glioblastoma cells. J Med Virol. 1996;49(3):205-211. doi:10.1002/(SICI)1096-

9071(199607)49:3<205::AID-JMV8>3.0.CO;2-8

179. Zorniak M, Clark PA, Leeper HE, Tipping MD, Francis DM, Kozak KR, Salamat MS, Kuo JS. Differential expression of 2',3'-cyclic-nucleotide 3'-phosphodiesterase and neural lineage markers correlate with glioblastoma xenograft infiltration and patient survival. Clin Cancer Res. 2012;18(13):3628-3636. doi:10.1158/1078-0432.CCR-12-0339

180. Skubal M, Gielen GH, Waha A, Gessi M, Kaczmarczyk L, Seifert G,

Freihoff D, Freihoff J, Pietsch T, Simon M et al. Altered splicing leads to 
medRxiv preprint doi: https://doi.org/10.1101/2020.12.21.20248616; this version posted December 23, 2020. The copyright holder for this preprint (which was not certified by peer review) is the author/funder, who has granted medRxiv a license to display the preprint in perpetuity. All rights reserved. No reuse allowed without permission.

reduced activation of CPEB3 in high-grade gliomas. Oncotarget. 2016;7(27):41898-41912. doi:10.18632/oncotarget.9735

181. Qu M, Yu J, Liu H, Ren Y, Ma C, Bu X, Lan Q. The Candidate Tumor Suppressor Gene SLC8A2 Inhibits Invasion, Angiogenesis and Growth of Glioblastoma. Mol Cells. 2017;40(10):761-772. doi:10.14348/molcells.2017.0104

182. Phillips E, Lang V, Bohlen J, Bethke F, Puccio L, Tichy D, Herold $\square$ Mende C, Hielscher T, Lichter P, Goidts V. Targeting atypical protein kinase $\mathrm{C}$ iota reduces viability in glioblastoma stem-like cells via a notch signaling mechanism. Int $\mathbf{J}$ Cancer. 2016;139(8):1776-1787. doi:10.1002/ijc.30234

183. Schulze M, Violonchi C, Swoboda S, Welz T, Kerkhoff E, Hoja S, Brüggemann S, Simbürger J, Reinders J, Riemenschneider MJ. RELN signaling modulates glioblastoma growth and substrate-dependent migration. Brain Pathol. 2018;28(5):695-709. doi:10.1111/bpa.12584

184. Han M, Wang S, Yang N, Wang X, Zhao W, Saed HS, Daubon T, Huang B, Chen A, Li G, et al. herapeutic implications of altered cholesterol homeostasis mediated by loss of CYP46A1 in human glioblastoma. EMBO Mol Med. 2019;e10924. doi:10.15252/emmm.201910924

185. Gao YF, Mao XY, Zhu T, Mao CX, Liu ZX, Wang ZB, Li L, Li X, Yin JY, Zhang W, et al. COL3A1 and SNAP91: novel glioblastoma markers with diagnostic and prognostic value. Oncotarget. 2016;7(43):70494-70503. doi:10.18632/oncotarget.12038

186. Guo Y, Zhang P, Zhang H, Zhang P, Xu R. RNAi for contactin 2 inhibits proliferation of U87-glioma stem cells by downregulating AICD, EGFR, and HES1. Onco Targets Ther. 2017;10:791-801. doi:10.2147/OTT.S113390

187. Grouzmann E, Meyer C, Bürki E, Brunner H. Neuropeptide Y Y2 receptor signalling mechanisms in the human glioblastoma cell line LN319. Peptides. 2001;22(3):379-386. doi:10.1016/s0196-9781(01)00344-8

188. Bao MH, Lv QL, Szeto V, Wong R, Zhu SZ, Zhang YY, Feng ZP, Sun HS. TRPM2-AS inhibits the growth, migration, and invasion of gliomas through JNK, c-Jun, and RGS4 [published online ahead of print, 2019 Oct 21]. J Cell Physiol. 2019;10.1002/jcp.29336. doi:10.1002/jcp.29336 
medRxiv preprint doi: https://doi.org/10.1101/2020.12.21.20248616; this version posted December 23, 2020. The copyright holder for this preprint (which was not certified by peer review) is the author/funder, who has granted medRxiv a license to display the preprint in perpetuity. All rights reserved. No reuse allowed without permission.

189. McAvoy S, Ganapathiraju S, Perez DS, James CD, Smith DI. DMD and IL1RAPL1: two large adjacent genes localized within a common fragile site (FRAXC) have reduced expression in cultured brain tumors. Cytogenet Genome Res. 2007;119(3-4):196-203. doi:10.1159/000112061

190. Song K, Yuan Y, Lin Y, Wang YX, Zhou J, Gai QJ, Zhang L, Mao M, Yao XX, Qin Y, et al. ERBB3, IGF1R, and TGFBR2 expression correlate with PDGFR expression in glioblastoma and participate in PDGFR inhibitor resistance of glioblastoma cells. Am J Cancer Res. 2018;8(5):792809.

191. Zhu Y, Zhang X, Wang L, Ji Z, Xie M, Zhou X, Liu Z, Shi H, Yu R. Loss of SH3GL2 promotes the migration and invasion behaviours of glioblastoma cells through activating the STAT3/MMP2 signalling. J Cell Mol Med. 2017;21(11):2685-2694. doi:10.1111/jcmm.13184

192. Delic S, Lottmann N, Jetschke K, Reifenberger G, Riemenschneider MJ. Identification and functional validation of CDH11, PCSK6 and SH3GL3 as novel glioma invasion-associated candidate genes. Neuropathol Appl Neurobiol. 2012;38(2):201-212. doi:10.1111/j.13652990.2011.01207.X

193. Zhang YX, Li XF, Yuan GQ, Hu H, Song XY, Li JY, Miao XK, Zhou TX, Yang WL, Zhang XW, et al. $\beta$-Arrestin 1 has an essential role in neurokinin-1 receptor-mediated glioblastoma cell proliferation and G2/M phase transition. J Biol Chem. 2017;292(21):8933-8947. doi:10.1074/jbc.M116.770420

194. Yang JK, Song J, Huo HR, Zhao YL, Zhang GY, Zhao ZM, Sun GZ, Jiao BH. DNM3, p65 and p53 from exosomes represent potential clinical diagnosis markers for glioblastoma multiforme. Ther Adv Med Oncol. 2017;9(12):741-754. doi:10.1177/1758834017737471

195. Yu F, Li G, Gao J, Sun Y, Liu P, Gao H, Li P, Lei T, Chen Y, Cheng $\mathrm{Y}$, et al. SPOCK1 is upregulated in recurrent glioblastoma and contributes to metastasis and Temozolomide resistance. Cell Prolif. 2016;49(2):195-206. doi:10.1111/cpr.12241

196. Oikonomou E, Buchfelder M, Adams EF. Cholecystokinin (CCK) and CCK receptor expression by human gliomas: Evidence for an autocrine/paracrine stimulatory loop. Neuropeptides. 2008;42(3):255-265. doi:10.1016/j.npep.2008.02.005 
medRxiv preprint doi: https://doi.org/10.1101/2020.12.21.20248616; this version posted December 23, 2020. The copyright holder for this

197. Suh JH, Park CK, Park SH. Alpha internexin expression related with molecular characteristics in adult glioblastoma and oligodendroglioma. J Korean Med Sci. 2013;28(4):593-601. doi:10.3346/jkms.2013.28.4.593

198. Igci YZ, Bozgeyik E, Borazan E, Pala E, Suner A, Ulasli M, Gurses SA, Yumrutas O, Balik AA, Igci M. Expression profiling of SCN8A and NDUFC2 genes in colorectal carcinoma. Exp Oncol. 2015;37(1):77-80.

199. Wang H, Liu XB, Chen JH, Wang QQ, Chen JP, Xu JF, Sheng CY, Ni QC. Decreased expression and prognostic role of cytoplasmic BRSK1 in human breast carcinoma: correlation with Jab1 stability and PI3K/Akt pathway. Exp Mol Pathol. 2014;97(2):191-201. doi:10.1016/j.yexmp.2014.07.012

200. Eckel-Passow JE, Serie DJ, Bot BM, Joseph RW, Cheville JC, Parker AS. ANKS1B is a smoking-related molecular alteration in clear cell renal cell carcinoma. BMC Urol. 2014;14:14. doi:10.1186/1471-2490-14-14

201. Stevenson L, Allen WL, Proutski I, Stewart G, Johnston L, McCloskey K, Wilson PM, Longley DB, Johnston PG. Calbindin 2 (CALB2) regulates 5-fluorouracil sensitivity in colorectal cancer by modulating the intrinsic apoptotic pathway. PLoS One. 2011;6(5):e20276. doi:10.1371/journal.pone.0020276

202. Chen A, Wang L, Li BY, Sherman J, Ryu JE, Hamamura K, Liu Y, Nakshatri H, Yokota H. Reduction in Migratory Phenotype in a Metastasized Breast Cancer Cell Line via Downregulation of S100A4 and GRM3. Sci Rep. 2017;7(1):3459. doi:10.1038/s41598-017-03811-9

203. Correa RG, de Carvalho AF, Pinheiro NA, Simpson AJ, de Souza SJ. NABC1 (BCAS1): alternative splicing and downregulation in colorectal tumors. Genomics. 2000;65(3):299-302. doi:10.1006/geno.2000.6172

204. Chen H, Liu Y, Jiang CJ, Chen YM, Li H, Liu QA. CalciumActivated Chloride Channel A4 (CLCA4) Plays Inhibitory Roles in Invasion and Migration Through Suppressing Epithelial-Mesenchymal Transition via PI3K/AKT Signaling in Colorectal Cancer. Med Sci Monit. 2019;25:41764185. doi:10.12659/MSM.914195

205. Sun Y, Ye D, Li Y, Chen E, Hao R, Cai Y, Wang Q, Wang O, Zhang $\mathrm{X}$. CUX2 functions as an oncogene in papillary thyroid cancer. Onco Targets Ther. 2018;12:217-224. doi:10.2147/OTT.S185710 
medRxiv preprint doi: https://doi.org/10.1101/2020.12.21.20248616; this version posted December 23, 2020. The copyright holder for this preprint (which was not certified by peer review) is the author/funder, who has granted medRxiv a license to display the preprint in perpetuity. All rights reserved. No reuse allowed without permission.

206. Yan H, Zheng C, Li Z, Bao B, Yang B, Hou K, Qu X, Xiao J, Che X, Liu Y. NPTX1 promotes metastasis via integrin/FAK signaling in gastric cancer. Cancer Manag Res. 2019;11:3237-3251. doi:10.2147/CMAR.S196509

207. Bong AHL, Robitaille M, Milevskiy MJG, Roberts-Thomson SJ, Monteith GR. NCS-1 expression is higher in basal breast cancers and regulates calcium influx and cytotoxic responses to doxorubicin. Mol Oncol. 2019. doi:10.1002/1878-0261.12589

208. Jeon TW, Yang H, Lee CG, Oh ST, Seo D, Baik IH, Lee EH, Yun I, Park KR, Lee YH. Electro-hyperthermia up-regulates tumour suppressor Septin 4 to induce apoptotic cell death in hepatocellular carcinoma. Int J Hyperthermia. 2016;32(6):648-656. doi:10.1080/02656736.2016.1186290

209. Chen J, Wu D, Zhang Y, Yang Y, Duan Y, An Y. LncRNA DCST1AS1 functions as a competing endogenous RNA to regulate FAIM2 expression by sponging miR-1254 in hepatocellular carcinoma. Clin Sci (Lond). 2019;133(2):367-379. doi:10.1042/CS20180814

210. Ou Y, Zheng X, Gao Y, Shu M, Leng T, Li Y, Yin W, Zhu W, Huang Y, Zhou Y, et al. Activation of cyclic AMP/PKA pathway inhibits bladder cancer cell invasion by targeting MAP4-dependent microtubule dynamics. Urol Oncol. 2014;32(1):47.e21-47.e4.7E28.

doi:10.1016/j.urolonc.2013.06.017

211. Zhao L, Xue M, Zhang L, Guo B, Qin Y, Jiang Q, Sun R, Yang J, Wang L, Liu L, et al. MicroRNA-4268 inhibits cell proliferation via AKT/JNK signalling pathways by targeting Rab6B in human gastric cancer. Cancer Gene Ther. 2019. doi:10.1038/s41417-019-0118-6

212. Bhandari A, Shen Y, Sindan N, Xia E, Gautam B, Lv S, Zhang X. MAL2 promotes proliferation, migration, and invasion through regulating epithelial-mesenchymal transition in breast cancer cell lines. Biochem Biophys Res Commun. 2018;504(2):434-439. doi:10.1016/j.bbrc.2018.08.187

213. Ye YP, Jiao HL, Wang SY, Xiao ZY, Zhang D, Qiu JF, Zhang LJ, Zhao YL, Li TT, Liao WT, et al. Hypermethylation of DMTN promotes the metastasis of colorectal cancer cells by regulating the actin cytoskeleton through Rac1 signaling activation. J Exp Clin Cancer Res. 2018;37(1):299. doi:10.1186/s13046-018-0958-1 
medRxiv preprint doi: https://doi.org/10.1101/2020.12.21.20248616; this version posted December 23, 2020. The copyright holder for this preprint (which was not certified by peer review) is the author/funder, who has granted medRxiv a license to display the preprint in perpetuity. All rights reserved. No reuse allowed without permission.

214. Liu YS, Lin HY, Lai SW, Huang CY, Huang BR, Chen PY, Wei KC, Lu DY. MiR-181b modulates EGFR-dependent VCAM-1 expression and monocyte adhesion in glioblastoma. MiR-181b modulates EGFR-dependent VCAM-1 expression and monocyte adhesion in glioblastoma. Oncogene. 2017;36(35):5006-5022. doi:10.1038/onc.2017.129

215. Luan W, Wang Y, Chen X, Shi Y, Wang J, Zhang J, Qian J, Li R, Tao $\mathrm{T}$, Wei $\mathrm{W}$, et al. PKM2 promotes glucose metabolism and cell growth in gliomas through a mechanism involving a let-7a/c-Myc/hnRNPA1 feedback loop. Oncotarget. 2015;6(15):13006-13018. doi:10.18632/oncotarget.3514

216. Zhu H, Chen D, Tang J, Huang C, Lv S, Wang D, Li G. Overexpression of centrosomal protein 55 regulates the proliferation of glioma cell and mediates proliferation promoted by EGFRvIII in glioblastoma U251 cells. Oncol Lett. 2018;15(2):2700-2706. doi:10.3892/ol.2017.7573

217. Keohane ME, Hall SW, VandenBerg SR, Gonias SL. Secretion of alpha 2-macroglobulin, alpha 2-antiplasmin, and plasminogen activator inhibitor-1 by glioblastoma multiforme in primary organ culture. J Neurosurg. 1990;73(2):234-241. doi:10.3171/jns.1990.73.2.0234

218. Koessinger D, Albrecht V, Faber F, Jaehnert I, Schichor C. ETS-1 Expression Is Hypoxia-independent in Glioblastoma-derived Endothelial and Mesenchymal Stem-like Cells. Anticancer Res. 2018;38(6):3347-3355. doi:10.21873/anticanres.12601

219. Kim SK, Kim K, Ryu JW, Ryu TY, Lim JH, Oh JH, Min JK, Jung CR, Hamamoto R, Son MY, et al. The novel prognostic marker, EHMT2, is involved in cell proliferation via HSPD1 regulation in breast cancer. Int J Oncol. 2019;54(1):65-76. doi:10.3892/ijo.2018.4608

220. Chung IC, Chen LC, Chung AK, Chao M, Huang HY, Hsueh C, Tsang NM, Chang KP, Liang Y, Li HP, et al. Matrix metalloproteinase 12 is induced by heterogeneous nuclear ribonucleoprotein $\mathrm{K}$ and promotes migration and invasion in nasopharyngeal carcinoma. BMC Cancer. 2014;14:348. doi:10.1186/1471-2407-14-348

221. Xu J, Zhu C, Yu Y, Wu W, Cao J, Li Z, Dai J, Wang C, Tang Y, Zhu $\mathrm{Q}$, et al. Systematic cancer-testis gene expression analysis identified CDCA5 as a potential therapeutic target in esophageal squamous cell carcinoma. EBioMedicine. 2019;46:54-65. doi:10.1016/j.ebiom.2019.07.030 
medRxiv preprint doi: https://doi.org/10.1101/2020.12.21.20248616; this version posted December 23, 2020. The copyright holder for this preprint (which was not certified by peer review) is the author/funder, who has granted medRxiv a license to display the preprint in perpetuity. All rights reserved. No reuse allowed without permission.

222. Takane K, Midorikawa Y, Yagi K, Sakai A, Aburatani H, Takayama T, Kaneda A. Aberrant promoter methylation of PPP1R3C and EFHD1 in plasma of colorectal cancer patients. Cancer Med. 2014;3(5):1235-1245. doi:10.1002/cam4.273

223. Yu C, Cao H, He X, Sun P, Feng Y, Chen L, Gong H. Cyclindependent kinase inhibitor $3(\mathrm{CDKN} 3)$ plays a critical role in prostate cancer via regulating cell cycle and DNA replication signaling. Biomed Pharmacother. 2017;96:1109-1118. doi:10.1016/j.biopha.2017.11.112

224. Li J, Ying Y, Xie H, Jin K, Yan H, Wang S, Xu M, Xu X, Wang X, Yang K, et al. Dual regulatory role of CCNA2 in modulating CDK6 and MET-mediated cell-cycle pathway and EMT progression is blocked by miR381-3p in bladder cancer. FASEB J. 2019;33(1):1374-1388. doi:10.1096/fj.201800667R

225. Hua K, Jin J, Zhang H, Zhao B, Wu C, Xu H, Fang L. MicroRNA-7 inhibits proliferation, migration and invasion of thyroid papillary cancer cells via targeting CKS2. Int J Oncol. 2016;49(4):1531-1540. doi:10.3892/ijo.2016.3660

226. Kim HE, Kim DG, Lee KJ, Son JG, Song MY, Park YM, Kim JJ, Cho SW, Chi SG, Cheong HS, et al. Frequent amplification of CENPF, GMNN and CDK13 genes in hepatocellular carcinomas. PLoS One. 2012;7(8):e43223. doi:10.1371/journal.pone.0043223

227. Li J, Liu Q, Liu Z, Xia Q, Zhang Z, Zhang R, Gao T, Gu G, Wang Y, Wang D, et al. KPNA2 promotes metabolic reprogramming in glioblastomas by regulation of c-myc. J Exp Clin Cancer Res. 2018;37(1):194. doi:10.1186/s13046-018-0861-9

228. Lei X, Deng L, Liu D, Liao S, Dai H, Li J, Rong J, Wang Z, Huang G,

Tang C, et al. ARHGEF7 promotes metastasis of colorectal adenocarcinoma by regulating the motility of cancer cells. Int J Oncol. 2018;53(5):19801996. doi:10.3892/ijo.2018.4535

229. Lescarbeau RS, Lei L, Bakken KK, Sims PA, Sarkaria JN, Canoll P,

White FM. Quantitative Phosphoproteomics Reveals Wee1 Kinase as a

Therapeutic Target in a Model of Proneural Glioblastoma. Mol Cancer Ther. 2016;15(6):1332-1343. doi:10.1158/1535-7163.MCT-15-0692

230. Bittencourt LF, Negreiros-Lima GL, Sousa LP, Silva AG, Souza IB, Ribeiro RI, Dutra MF, Silva RF, Dias AC, Soriani FM, et al. Correction to: 
medRxiv preprint doi: https://doi.org/10.1101/2020.12.21.20248616; this version posted December 23, 2020. The copyright holder for this

G3BP1 knockdown sensitizes U87 glioblastoma cell line to Bortezomib by inhibiting stress granules assembly and potentializing apoptosis. J Neurooncol. 2019;144(3):475. doi:10.1007/s11060-019-03276-y

231. Wang JZ, Yang SX, Ye F, Xia XP, Shao XX, Xia SL, Zheng B, Xu

CL. Hypoxia-induced Rab11-family interacting protein 4 expression promotes migration and invasion of colon cancer and correlates with poor prognosis. Mol Med Rep. 2018;17(3):3797-3806. doi:10.3892/mmr.2017.8283

232. Pellegatta S, Ianni ND, Pessina S, Paterra R, Anghileri E, Eoli M, Finocchiaro G. ABCC3 Expressed by CD56dim CD16+ NK Cells Predicts Response in Glioblastoma Patients Treated with Combined Chemotherapy and Dendritic Cell Immunotherapy. Int J Mol Sci. 2019;20(23):E5886. doi:10.3390/ijms20235886

233. Soichi O, Masanori N, Hideo T, Kazunori A, Nobuya I, Jun-ichi K. Clinical significance of ABCA2' a possible molecular marker for oligodendrogliomas. Neurosurgery. 2007;60(4):707-714. doi:10.1227/01.NEU.0000255395.15657.06

234. Yang J, Min KW, Kim DH, Son BK, Moon KM, Wi YC, Bang SS, Oh YH, Do SI, Chae SW, et al. . High TNFRSF12A level associated with MMP-9 overexpression is linked to poor prognosis in breast cancer: Gene set enrichment analysis and validation in large-scale cohorts. PLoS One. 2018;13(8):e0202113. doi:10.1371/journal.pone.0202113

235. Zhou J, Wang H, Lu A, Hu G, Luo A, Ding F, Zhang J, Wang X, Wu M, Liu Z. A novel gene, NMES1, downregulated in human esophageal squamous cell carcinoma. Int J Cancer. 2002;101(4):311-316. doi:10.1002/ijc. 10600

236. Aguilar-Morante D, Morales-Garcia JA, Santos A, Perez-Castillo A. CCAAT/enhancer binding protein $\beta$ induces motility and invasion of glioblastoma cells through transcriptional regulation of the calcium binding protein S100A4. Oncotarget. 2015;6(6):4369-4384. doi:10.18632/oncotarget.2976

237. Chen L, Gong X, Huang M. YY1-Activated Long Noncoding RNA SNHG5 Promotes Glioblastoma Cell Proliferation Through p38/MAPK Signaling Pathway. Cancer Biother Radiopharm. 2019;34(9):589-596. doi:10.1089/cbr.2019.2779 
medRxiv preprint doi: https://doi.org/10.1101/2020.12.21.20248616; this version posted December 23, 2020. The copyright holder for this preprint (which was not certified by peer review) is the author/funder, who has granted medRxiv a license to display the preprint in perpetuity. All rights reserved. No reuse allowed without permission.

238. Raja R, Sahasrabuddhe NA, Radhakrishnan A, Syed N, Solanki HS, Puttamallesh VN, Balaji SA, Nanjappa V, Datta KK, Babu N, et al. Chronic exposure to cigarette smoke leads to activation of p21 (RAC1)-activated kinase 6 (PAK6) in non-small cell lung cancer cells. Oncotarget. 2016;7(38):61229-61245. doi:10.18632/oncotarget.11310

239. Kundu S, Ramshankar V, Verma AK, Thangaraj SV, Krishnamurthy A, Kumar R, Kannan R, Ghosh SK. Association of DFNA5, SYK, and NELL1 variants along with HPV infection in oral cancer among the prolonged tobacco-chewers. Tumour Biol. 2018;40(8):1010428318793023. doi: $10.1177 / 1010428318793023$

\section{Tables}

Table 1 The statistical metrics for key differentially expressed genes (DEGs)

\begin{tabular}{|c|c|c|c|c|c|c|c|}
\hline Illumina Id & Gene Symbol & $\log \mathrm{C}$ & P Value & $\begin{array}{c}\text { Adjusted } \\
\text { P Value } \\
\end{array}$ & $t$ value & Regulation & Gene Name \\
\hline ILMN_1788874 & SERPINA3 & 4.06653 & $1.06 \mathrm{E}-15$ & $1.43 \mathrm{E}-12$ & 12.61288 & Up & serpin family A member 3 \\
\hline ILMN_3293676 & AC015911.1 & 2.063268 & $1.24 \mathrm{E}-15$ & $1.54 \mathrm{E}-12$ & 12.55419 & Up & ribosomal protein L39 (RPL39) pseudogene \\
\hline ILMN_2409167 & ANXA2 & 3.255975 & $4.65 \mathrm{E}-15$ & $3.18 \mathrm{E}-12$ & 12.05193 & Up & annexin $\mathrm{A} 2$ \\
\hline ILMN_3251341 & TUBA1C & 3.365133 & $7.2 \mathrm{E}-15$ & $4.19 \mathrm{E}-12$ & 11.88888 & Up & tubulin alpha $1 \mathrm{c}$ \\
\hline ILMN_1693421 & RPN2 & 2.816487 & $1.08 \mathrm{E}-14$ & $5.38 \mathrm{E}-12$ & 11.73836 & Up & ribophorin II \\
\hline ILMN_1653028 & COL4A1 & 3.383137 & $2.06 \mathrm{E}-14$ & $7.98 \mathrm{E}-12$ & 11.502 & Up & $\begin{array}{l}\text { collagen type IV alpha } 1 \text { chain } \\
\text { CKLF like MARVEL transmembrane domain }\end{array}$ \\
\hline ILMN_1705442 & СМтМз & 1.900321 & $2.25 \mathrm{E}-14$ & $8.56 \mathrm{E}-12$ & 11.47019 & Up & $\begin{array}{l}\text { containing } 3 \\
\text { KDEL endoplasmic reticulum protein retention }\end{array}$ \\
\hline ILMN_1724293 & KDELR2 & 2.302602 & $2.31 \mathrm{E}-14$ & 8.67E-12 & 11.45963 & Up & receptor 2 \\
\hline ILMN_2041101 & ANXA2P1 & 4.608619 & $3.3 \mathrm{E}-14$ & 1.17E-11 & 11.33084 & Up & annexin A2 pseudogene 1 \\
\hline ILMN_1756982 & CLIC1 & 2.316807 & $5.49 \mathrm{E}-14$ & $1.76 \mathrm{E}-11$ & 11.14768 & Up & $\begin{array}{l}\text { chloride intracellular channel } 1 \\
\text { oligosaccharyltransferase complex non-catalytic }\end{array}$ \\
\hline ILMN_2056167 & OSTC & 2.986182 & $1.1 \mathrm{E}-13$ & 3.07E-11 & 10.89956 & Up & subunit \\
\hline ILMN_3211132 & RPS2P48 & 2.261457 & $1.41 \mathrm{E}-13$ & $3.74 \mathrm{E}-11$ & 10.81191 & Up & ribosomal protein $\mathrm{S} 2$ pseudogene 48 \\
\hline ILMN_1803429 & CD44 & 3.080897 & $1.44 \mathrm{E}-13$ & $3.79 \mathrm{E}-11$ & 10.80486 & Up & CD44 molecule (Indian blood group) \\
\hline ILMN_1711566 & TIMP1 & 3.422648 & $1.47 \mathrm{E}-13$ & $3.86 \mathrm{E}-11$ & 10.79694 & Up & TIMP metallopeptidase in hibitor 1 \\
\hline ILMN_1714759 & $\mathrm{CNIH} 4$ & 2.460108 & $1.99 \mathrm{E}-13$ & $4.6 \mathrm{E}-11$ & 10.69074 & Up & cornichon family AMPA receptor auxiliary protein 4 \\
\hline ILMN_2090105 & TAGLN2 & 2.292586 & $2.14 \mathrm{E}-13$ & $4.82 \mathrm{E}-11$ & 10.66425 & Up & transgelin 2 \\
\hline ILMN_1782538 & VIM & 2.25539 & $3.27 \mathrm{E}-13$ & $6.51 \mathrm{E}-11$ & 10.51611 & Up & vimentin \\
\hline ILMN_1810852 & LAMC1 & 2.006069 & $4.76 \mathrm{E}-13$ & $8.79 \mathrm{E}-11$ & 10.38613 & Up & laminin subunit gamma 1 \\
\hline ILMN_3243441 & EEF1A1 & 2.002447 & $4.92 \mathrm{E}-13$ & $9 \mathrm{E}-11$ & 10.37415 & Up & eukaryotic translation elongation factor 1 alpha 1 \\
\hline ILMN_2110908 & MYC & 2.800608 & $5.53 \mathrm{E}-13$ & $9.75 \mathrm{E}-11$ & 10.33388 & Up & MYC proto-oncogene, bHLH transcription factor \\
\hline ILMN_2140059 & LAMA4 & 2.730971 & $5.6 \mathrm{E}-13$ & $9.83 \mathrm{E}-11$ & 10.32956 & Up & $\begin{array}{l}\text { laminin subunit alpha } 4 \\
\text { mitochondrial calcium uniporter dominant negative }\end{array}$ \\
\hline ILMN_1801766 & MCUB & 2.454932 & $6.36 \mathrm{E}-13$ & $1.08 \mathrm{E}-10$ & 10.28585 & Up & beta subunit \\
\hline ILMN_1665559 & CDK2 & 2.088775 & $7.6 \mathrm{E}-13$ & $1.26 \mathrm{E}-10$ & 10.22438 & Up & cyclin dependent kinase 2 \\
\hline ILMN_2058251 & VIM & 2.46105 & $9.25 \mathrm{E}-13$ & $1.46 \mathrm{E}-10$ & 10.15679 & Up & vimentin \\
\hline ILMN_2104106 & XPR1 & 2.033177 & $9.62 \mathrm{E}-13$ & $1.5 \mathrm{E}-10$ & 10.14325 & Up & xenotropic and polytropic retrovirus receptor 1 \\
\hline
\end{tabular}


medRxiv preprint doi: https://doi.org/10.1101/2020.12.21.20248616; this version posted December 23, 2020. The copyright holder for this preprint (which was not certified by peer review) is the author/funder, who has granted medRxiv a license to display the preprint in perpetuity. All rights reserved. No reuse allowed without permission.

\begin{tabular}{|c|c|c|c|c|c|c|c|}
\hline ILMN_2222234 & PRDX4 & 2.124939 & $1.41 \mathrm{E}-12$ & $2.06 \mathrm{E}-10$ & 10.01329 & Up & peroxiredoxin 4 \\
\hline ILMN_1755115 & RPL23 & 2.458215 & $2.05 \mathrm{E}-12$ & $2.8 \mathrm{E}-10$ & 9.884818 & Up & ribosomal protein $\mathrm{L} 23$ \\
\hline ILMN_2302757 & FCGBP & 3.361262 & $2.17 \mathrm{E}-12$ & $2.93 \mathrm{E}-10$ & 9.866115 & Up & Fc fragment of lgG binding protein \\
\hline ILMN_3268914 & NPM1P34 & 2.444988 & $2.18 \mathrm{E}-12$ & $2.93 \mathrm{E}-10$ & 9.864896 & Up & nucleophosmin 1 pseudogene 34 \\
\hline ILMN_1774207 & ANGPT2 & 2.903619 & $2.19 \mathrm{E}-12$ & $2.94 \mathrm{E}-10$ & 9.863178 & Up & angiopoietin 2 \\
\hline ILMN_3287093 & RPS15A & 2.061838 & $2.52 \mathrm{E}-12$ & $3.26 \mathrm{E}-10$ & 9.815719 & Up & ribosomal protein $\mathrm{S15a}$ \\
\hline ILMN_1700896 & SAP3O & 2.088787 & $2.78 \mathrm{E}-12$ & $3.48 \mathrm{E}-10$ & 9.782203 & Up & $\operatorname{Sin} 3 \mathrm{~A}$ associated protein 30 \\
\hline ILMN_1803788 & LGALS3 & 3.453401 & $2.86 \mathrm{E}-12$ & $3.53 \mathrm{E}-10$ & 9.773362 & Up & galectin 3 \\
\hline ILMN_3213573 & EEF1A1 & 2.161518 & $2.86 \mathrm{E}-12$ & $3.53 \mathrm{E}-10$ & 9.772742 & Up & eukaryotic translation elongation factor 1 alpha 1 \\
\hline ILMN_2336781 & SOD2 & 3.242982 & $3.31 \mathrm{E}-12$ & $3.96 \mathrm{E}-10$ & 9.723934 & Up & superoxide dismutase 2 \\
\hline ILMN_3233388 & RELL1 & 2.073019 & $3.42 \mathrm{E}-12$ & $4.05 \mathrm{E}-10$ & 9.712494 & Up & RELT like 1 \\
\hline ILMN_2049021 & PTTG3P & 3.145237 & $3.87 \mathrm{E}-12$ & 4.45E-10 & 9.671245 & Up & pituitary tumor-transforming 3 , pseudogene \\
\hline ILMN_1663866 & TGFBI & 4.177962 & $3.93 \mathrm{E}-12$ & $4.51 \mathrm{E}-10$ & 9.665767 & Up & transforming growth factor beta induced \\
\hline ILMN_1696187 & PYGL & 1.992241 & $4.37 \mathrm{E}-12$ & $4.87 \mathrm{E}-10$ & 9.630502 & Up & glycogen phosphorylase L \\
\hline ILMN_1706117 & RPS15A & 2.084669 & $4.46 \mathrm{E}-12$ & $4.91 \mathrm{E}-10$ & 9.623167 & Up & ribosomal protein $\mathrm{S} 15 \mathrm{a}$ \\
\hline ILMN_1714861 & CD68 & 2.062473 & $4.71 \mathrm{E}-12$ & $5.07 \mathrm{E}-10$ & 9.60531 & Up & CD68 molecule \\
\hline ILMN_3239629 & RPS2P32 & 2.691414 & $5.4 \mathrm{E}-12$ & $5.68 \mathrm{E}-10$ & 9.559264 & Up & ribosomal protein \$2 pseudogene 32 \\
\hline ILMN_3205656 & GAPDH & 2.293028 & $5.63 \mathrm{E}-12$ & $5.87 \mathrm{E}-10$ & 9.545775 & Up & $\begin{array}{l}\text { glyceraldehyde-3-phosphate dehydrogenase } \\
\text { PTTG1 regulator of sister chromatid separation, }\end{array}$ \\
\hline ILMN_2042771 & PTTG1 & 3.21267 & $5.66 \mathrm{E}-12$ & $5.89 \mathrm{E}-10$ & 9.543928 & Up & securin \\
\hline ILMN_2340935 & TCEAL9 & 2.156234 & $6.09 \mathrm{E}-12$ & $6.26 \mathrm{E}-10$ & 9.519457 & Up & transcription elongation factor $\mathrm{A}$ like 9 \\
\hline ILMN_3279219 & SNRPG & 2.252904 & $6.54 \mathrm{E}-12$ & $6.64 \mathrm{E}-10$ & 9.4956 & Up & small nuclear ribonucleoprotein polypeptide $G$ \\
\hline ILMN_3224907 & EEF1A1 & 2.618298 & $7.01 \mathrm{E}-12$ & $7.05 \mathrm{E}-10$ & 9.472314 & Up & eukaryotic translation elongation factor 1 alpha 1 \\
\hline ILMN_2122103 & ETS1 & 2.668099 & $7.66 \mathrm{E}-12$ & $7.58 \mathrm{E}-10$ & 9.442879 & Up & ETS proto-oncogene 1 , transcription factor \\
\hline ILMN_1742461 & UAP1 & 1.982771 & $1.09 \mathrm{E}-11$ & $1.01 \mathrm{E}-09$ & 9.327349 & Up & UDP-N-acetylglucosaminepyrophosphorylase 1 \\
\hline ILMN_1713636 & S100A6 & 1.8866 & $1.11 \mathrm{E}-11$ & $1.03 \mathrm{E}-09$ & 9.319517 & Up & S100 calcium binding protein $A 6$ \\
\hline ILMN_1726720 & NUSAP1 & 3.614245 & $1.12 \mathrm{E}-11$ & $1.03 E-09$ & 9.318064 & Up & nucleolar and spindle associated protein 1 \\
\hline ILMN_1694177 & PCNA & 2.618813 & $1.15 \mathrm{E}-11$ & $1.06 \mathrm{E}-09$ & 9.308388 & Up & proliferating cell nuclear antigen \\
\hline ILMN_1725193 & IGFBP2 & 4.137955 & $1.32 \mathrm{E}-11$ & $1.18 \mathrm{E}-09$ & 9.263546 & Up & insulin like growth factor binding protein 2 \\
\hline ILMN_3253304 & BRI3 & 2.203082 & $1.42 \mathrm{E}-11$ & $1.26 \mathrm{E}-09$ & 9.237632 & Up & brain protein 13 \\
\hline ILMN_2176037 & GNA13 & 2.319814 & $1.43 \mathrm{E}-11$ & $1.26 \mathrm{E}-09$ & 9.235716 & Up & G protein subunit alpha 13 \\
\hline ILMN_1814998 & POTEKP & 2.306258 & $1.94 \mathrm{E}-11$ & $1.61 \mathrm{E}-09$ & 9.136451 & Up & POTE ankyrin domain family member $K$, pseudogene \\
\hline ILMN_1773079 & COL3A1 & 3.779291 & $1.94 \mathrm{E}-11$ & $1.61 \mathrm{E}-09$ & 9.135782 & Up & collagen type III alpha 1 chain \\
\hline ILMN_1744611 & DCAF13 & 2.070568 & $1.97 \mathrm{E}-11$ & $1.63 \mathrm{E}-09$ & 9.131068 & Up & DDB1 and CUL4 associated factor 13 \\
\hline ILMN_1750101 & S100A11 & 2.495667 & $2.02 \mathrm{E}-11$ & 1.67E-09 & 9.122157 & Up & S100 calcium binding protein A11 \\
\hline ILMN_2148913 & TMEM45A & 2.919972 & $2.06 \mathrm{E}-11$ & $1.69 \mathrm{E}-09$ & 9.116505 & Up & transmembrane protein $45 \mathrm{~A}$ \\
\hline ILMN_1651228 & RPS28 & 2.462082 & $2.1 \mathrm{E}-11$ & $1.7 \mathrm{E}-09$ & 9.110125 & Up & ribosomal protein $\mathrm{S} 28$ \\
\hline ILMN_1660806 & CSRP2 & 2.395905 & $2.14 \mathrm{E}-11$ & $1.73 \mathrm{E}-09$ & 9.10383 & Up & cysteine and glycine rich protein 2 \\
\hline ILMN_2192694 & EIF3M & 2.397109 & $2.33 \mathrm{E}-11$ & $1.87 \mathrm{E}-09$ & 9.075482 & Up & eukaryotic translation initiation factor 3 subunit $M$ \\
\hline ILMN_2398388 & APH1A & 1.929527 & $2.34 \mathrm{E}-11$ & $1.87 \mathrm{E}-09$ & 9.073846 & Up & aph-1 homolog A, gamma-secretase subunit \\
\hline ILMN_2181540 & YY1 & 2.251239 & $2.46 \mathrm{E}-11$ & $1.95 \mathrm{E}-09$ & 9.057497 & Up & YY1 transcription factor \\
\hline ILMN_2301083 & UBE2C & 3.889327 & $2.61 \mathrm{E}-11$ & $2.04 \mathrm{E}-09$ & 9.037984 & Up & ubiquitin conjugating enzyme E2 C \\
\hline ILMN_1699489 & TUBB6 & 2.468045 & $2.7 \mathrm{E}-11$ & $2.09 \mathrm{E}-09$ & 9.026951 & Up & tubulin beta 6 class $V$ \\
\hline ILMN_1789702 & GBE1 & 2.058722 & $3.36 \mathrm{E}-11$ & $2.5 \mathrm{E}-09$ & 8.956208 & Up & 1,4-alpha-glucan branching enzyme 1 \\
\hline
\end{tabular}


medRxiv preprint doi: https://doi.org/10.1101/2020.12.21.20248616; this version posted December 23, 2020. The copyright holder for this preprint (which was not certified by peer review) is the author/funder, who has granted medRxiv a license to display the preprint in perpetuity. All rights reserved. No reuse allowed without permission.

\begin{tabular}{|c|c|c|c|c|c|c|c|}
\hline ILMN_1678454 & CASP4 & 2.122211 & $3.73 \mathrm{E}-11$ & 2.74E-09 & 8.922115 & Up & caspase 4 \\
\hline ILMN_2344455 & G3BP1 & 2.549132 & $3.9 E-11$ & 2.83E-09 & 8.907646 & Up & G3BP stress granule assembly factor 1 \\
\hline ILMN_2219712 & HMGB2 & 3.127116 & $4.04 \mathrm{E}-11$ & $2.92 \mathrm{E}-09$ & 8.89562 & Up & high mobility group box 2 \\
\hline ILMN_3226613 & CLEC2D & 1.991598 & $4.75 \mathrm{E}-11$ & $3.32 \mathrm{E}-09$ & 8.843193 & Up & C-type lectin domain family 2 member $D$ \\
\hline ILMN_1734814 & HSPA4 & 2.085002 & $4.8 \mathrm{E}-11$ & $3.35 \mathrm{E}-09$ & 8.84004 & Up & heat shock protein family A (Hsp70) member 4 \\
\hline ILMN_1676792 & RPS11 & 2.00015 & $5.23 \mathrm{E}-11$ & $3.59 \mathrm{E}-09$ & 8.812165 & Up & ribosomal protein S11 \\
\hline ILMN_1656386 & SEC24D & 2.086069 & $5.46 \mathrm{E}-11$ & $3.72 \mathrm{E}-09$ & 8.797993 & Up & SEC24 homolog D, COPII coat complex component \\
\hline ILMN_2322806 & CAST & 2.057064 & $5.52 \mathrm{E}-11$ & $3.76 \mathrm{E}-09$ & 8.79443 & Up & calpastatin \\
\hline ILMN_1755077 & HEBP2 & 2.231615 & $5.68 \mathrm{E}-11$ & 3.84E-09 & 8.785193 & Up & heme binding protein 2 \\
\hline ILMN_1716014 & RPL15 & 2.384932 & $5.9 \mathrm{E}-11$ & 3.96E-09 & 8.772838 & Up & ribosomal protein L15 \\
\hline ILMN_1686097 & TOP2A & 4.148793 & $6.16 \mathrm{E}-11$ & $4.08 \mathrm{E}-09$ & 8.75902 & Up & DNA topoisomerase II alpha \\
\hline ILMN_1784005 & RAB13 & 2.500981 & $6.37 \mathrm{E}-11$ & 4.21E-09 & 8.748159 & Up & RAB13, member RAS oncogene family \\
\hline ILMN_3250067 & ANGPT2 & 2.750754 & $8.24 \mathrm{E}-11$ & $5.22 \mathrm{E}-09$ & 8.665017 & Up & angiopoietin 2 \\
\hline ILMN_1725244 & HAT1 & 2.229167 & $8.44 \mathrm{E}-11$ & $5.33 \mathrm{E}-09$ & 8.657173 & Up & histone acetyltransferase 1 \\
\hline ILMN_1763539 & IER3IP1 & 2.045767 & $9.5 \mathrm{E}-11$ & 5.89E-09 & 8.618952 & Up & immediate early response 3 interacting protein 1 \\
\hline ILMN_1689004 & TNFRSF12A & 2.75761 & $9.56 \mathrm{E}-11$ & $5.91 \mathrm{E}-09$ & 8.616918 & Up & TNF receptor superfamily member $12 \mathrm{~A}$ \\
\hline ILMN_1704730 & CD93 & 2.266122 & $9.74 \mathrm{E}-11$ & $6 \mathrm{E}-09$ & 8.61107 & Up & CD93 molecule \\
\hline ILMN_1784948 & SPOCD1 & 3.532944 & $9.77 \mathrm{E}-11$ & $6.02 \mathrm{E}-09$ & 8.609932 & Up & SPOC domain containing 1 \\
\hline ILMN_3278506 & RPS2P32 & 2.140335 & $9.83 \mathrm{E}-11$ & 6.04E-09 & 8.608043 & Up & ribosomal protein S2 pseudogene 32 \\
\hline ILMN_1693334 & P4HA1 & 2.132018 & $1 \mathrm{E}-10$ & $6.13 \mathrm{E}-09$ & 8.602418 & Up & prolyl 4-hydroxylase subunit alpha 1 \\
\hline ILMN_1670490 & PDPN & 3.143628 & $1.05 \mathrm{E}-10$ & 6.37E-09 & 8.586602 & Up & podoplanin \\
\hline ILMN_1747016 & CEP55 & 2.616765 & $1.05 \mathrm{E}-10$ & $6.38 \mathrm{E}-09$ & 8.585722 & Up & centrosomal protein 55 \\
\hline ILMN_3279675 & RPS18 & 1.924299 & $1.11 \mathrm{E}-10$ & $6.59 \mathrm{E}-09$ & 8.570102 & Up & ribosomal protein $\mathrm{S} 18$ \\
\hline ILMN_2182120 & SF3B6 & 2.030975 & $1.13 \mathrm{E}-10$ & $6.66 \mathrm{E}-09$ & 8.564252 & Up & splicing factor $3 \mathrm{~b}$ subunit 6 \\
\hline ILMN_2379788 & HIF1A & 2.676848 & $1.21 \mathrm{E}-10$ & 7.04E-09 & 8.541742 & Up & hypoxia inducible factor 1 subunit alpha \\
\hline ILMN_1722532 & KDM3A & 1.880518 & $1.38 \mathrm{E}-10$ & 7.88E-09 & 8.49988 & Up & lysine demethylase $3 \mathrm{~A}$ \\
\hline ILMN_1742866 & $\mathrm{F} 2 \mathrm{R}$ & 2.340882 & $1.47 \mathrm{E}-10$ & $8.28 \mathrm{E}-09$ & 8.477838 & Up & coagulation factor II thrombin receptor \\
\hline ILMN_2072296 & CKS2 & 2.885023 & $1.47 \mathrm{E}-10$ & $8.28 \mathrm{E}-09$ & 8.477793 & Up & $\mathrm{CDC} 28$ protein kinase regulatory subunit 2 \\
\hline ILMN_1657153 & ACTR3 & 2.628862 & $1.53 \mathrm{E}-10$ & $8.52 \mathrm{E}-09$ & 8.465732 & Up & actin related protein 3 \\
\hline ILMN_2186806 & HLA-A & 3.230822 & $1.6 \mathrm{E}-10$ & $8.82 \mathrm{E}-09$ & 8.452009 & Up & major histocom patibility complex, class I, A \\
\hline ILMN_1714730 & UBE2C & 3.627574 & $1.64 \mathrm{E}-10$ & $9.03 \mathrm{E}-09$ & 8.443256 & Up & ubiquitin conjugating enzyme E2 C \\
\hline ILMN_3294222 & RPS2P32 & 3.856691 & $1.74 \mathrm{E}-10$ & $9.51 \mathrm{E}-09$ & 8.424433 & Up & ribosomal protein \$2 pseudogene 32 \\
\hline ILMN_3279414 & EEF1A1 & 2.544653 & $1.78 \mathrm{E}-10$ & $9.7 \mathrm{E}-09$ & 8.41696 & Up & eukaryotic translation elongation factor 1 alpha 1 \\
\hline ILMN_2386891 & GLT8D1 & 1.988499 & $1.82 \mathrm{E}-10$ & $9.84 \mathrm{E}-09$ & 8.411157 & Up & glycosyltransferase 8 domain containing 1 \\
\hline ILMN_3226291 & RPL29 & 2.122823 & $1.82 \mathrm{E}-10$ & $9.86 \mathrm{E}-09$ & 8.410117 & Up & ribosomal protein $\mathrm{L} 29$ \\
\hline ILMN_1735996 & NOX4 & 2.662736 & $1.87 \mathrm{E}-10$ & $1.01 \mathrm{E}-08$ & 8.400779 & Up & NADPH oxidase 4 \\
\hline ILMN_1785107 & NXT2 & 1.987026 & $1.89 \mathrm{E}-10$ & $1.01 \mathrm{E}-08$ & 8.398452 & Up & nuclear transport factor 2 like export factor 2 \\
\hline ILMN_1685378 & RPS7 & 2.487842 & $1.97 \mathrm{E}-10$ & $1.05 \mathrm{E}-08$ & 8.384299 & Up & ribosomal protein $\mathrm{S7}$ \\
\hline ILMN_1653871 & NAMPT & 2.888382 & 1.99E-10 & $1.06 \mathrm{E}-08$ & 8.381269 & Up & nicotinamidephosphoribosyltransferase \\
\hline ILMN_1751028 & SERPINH1 & 2.653259 & $2.19 \mathrm{E}-10$ & $1.15 \mathrm{E}-08$ & 8.351233 & Up & serpin family $\mathrm{H}$ member 1 \\
\hline ILMN_1656057 & PLAU & 2.952386 & $2.29 \mathrm{E}-10$ & $1.19 \mathrm{E}-08$ & 8.337367 & Up & plasminogen activator, urokinase \\
\hline ILMN_3298215 & RPL17P36 & 2.451302 & $2.41 \mathrm{E}-10$ & $1.24 \mathrm{E}-08$ & 8.320873 & Up & ribosomal protein L17 pseudogene 36 \\
\hline ILMN_1778561 & WEE1 & 2.450754 & $2.53 \mathrm{E}-10$ & $1.29 \mathrm{E}-08$ & 8.304904 & Up & WEE1 G2 checkpoint kinase \\
\hline
\end{tabular}


medRxiv preprint doi: https://doi.org/10.1101/2020.12.21.20248616; this version posted December 23, 2020. The copyright holder for this preprint (which was not certified by peer review) is the author/funder, who has granted medRxiv a license to display the preprint in perpetuity. All rights reserved. No reuse allowed without permission.

\begin{tabular}{|c|c|c|c|c|c|c|c|}
\hline ILMN_3290353 & RPS15A & 1.895235 & $2.6 \mathrm{E}-10$ & $1.31 \mathrm{E}-08$ & 8.296668 & Up & ribosomal protein $\mathrm{S15a}$ \\
\hline ILMN_3235148 & RPL23AP87 & 2.6731 & $2.69 \mathrm{E}-10$ & $1.35 \mathrm{E}-08$ & 8.285405 & Up & ribosomal protein L23a pseudogene 87 \\
\hline ILMN_2408415 & RPL9 & 2.45844 & $2.72 \mathrm{E}-10$ & $1.36 \mathrm{E}-08$ & 8.281846 & Up & ribosomal protein $\mathrm{L} 9$ \\
\hline ILMN_1695588 & HNRNPC & 2.218953 & $2.76 \mathrm{E}-10$ & $1.38 \mathrm{E}-08$ & 8.277424 & Up & heterogeneous nuclear ribonucleoprotein $C$ \\
\hline ILMN_1774077 & GBP2 & 2.517058 & $2.82 \mathrm{E}-10$ & $1.4 \mathrm{E}-08$ & 8.270108 & Up & guanylate binding protein 2 \\
\hline ILMN_3300471 & AC093422.1 & 2.640217 & $2.83 \mathrm{E}-10$ & $1.4 \mathrm{E}-08$ & 8.269253 & Up & ribosomal protein L17 (RPL17) pseudogene \\
\hline ILMN_3275345 & RPS27 & 2.454238 & $3.19 \mathrm{E}-10$ & $1.56 \mathrm{E}-08$ & 8.230957 & Up & ribosomal protein $\$ 27$ \\
\hline ILMN_3206132 & RPS8 & 2.916985 & $3.31 \mathrm{E}-10$ & $1.6 \mathrm{E}-08$ & 8.219665 & Up & ribosomal protein $\mathrm{S} 8$ \\
\hline ILMN_1676765 & RPL18A & 2.295485 & $3.41 \mathrm{E}-10$ & $1.64 \mathrm{E}-08$ & 8.210189 & Up & ribosomal protein L18a \\
\hline ILMN_3292056 & RPL17P22 & 2.452461 & $3.44 \mathrm{E}-10$ & $1.65 \mathrm{E}-08$ & 8.207372 & Up & ribosomal protein L17 pseudogene 22 \\
\hline ILMN_1726030 & GPX7 & 1.958802 & $3.75 \mathrm{E}-10$ & $1.77 \mathrm{E}-08$ & 8.179698 & Up & glutathione peroxidase 7 \\
\hline ILMN_2156953 & ZFAND6 & 1.890473 & $3.76 \mathrm{E}-10$ & $1.77 \mathrm{E}-08$ & 8.179429 & Up & zinc finger AN1-type containing 6 \\
\hline ILMN_2154836 & BTG3 & 2.70263 & $4.04 \mathrm{E}-10$ & $1.88 \mathrm{E}-08$ & 8.156574 & Up & BTG anti-proliferation factor 3 \\
\hline ILMN_1751816 & MCTS1 & 2.251603 & $4.05 \mathrm{E}-10$ & $1.89 \mathrm{E}-08$ & 8.155484 & Up & MCTS1 re-initiation and release factor \\
\hline ILMN_1747911 & CDK1 & 2.735175 & $4.22 \mathrm{E}-10$ & $1.96 \mathrm{E}-08$ & 8.142517 & Up & cyclin dependent kinase 1 \\
\hline ILMN_3271122 & RPS3A & 2.768008 & 4.26E-10 & $1.96 \mathrm{E}-08$ & 8.139922 & Up & ribosomal protein S3A \\
\hline ILMN_1652955 & RPS27 & 1.8972 & $4.39 \mathrm{E}-10$ & $2.02 \mathrm{E}-08$ & 8.129704 & Up & $\begin{array}{c}\text { ribosomal protein } \$ 27 \\
\text { CCZ1 homolog, vacuolar protein trafficking and }\end{array}$ \\
\hline ILMN_1660270 & CCZ1 & 2.368278 & 4.47E-10 & $2.05 \mathrm{E}-08$ & 8.124213 & Up & biogenesis associated \\
\hline ILMN_2357438 & AURKA & 2.223213 & 4.48E-10 & $2.05 \mathrm{E}-08$ & 8.123374 & Up & aurora kinase $\mathrm{A}$ \\
\hline ILMN_3290577 & AC109454.1 & 2.070226 & $4.68 \mathrm{E}-10$ & $2.13 \mathrm{E}-08$ & 8.109471 & Up & ribosomal protein S10 (RPS10) pseudogene \\
\hline ILMN_3267017 & RPLP1 & 3.06519 & 4.79E-10 & 2.17E-08 & 8.102576 & Up & ribosomal protein lateral stalk subunit P1 \\
\hline ILMN_1796712 & S100A10 & 2.818816 & $5.35 \mathrm{E}-10$ & $2.4 \mathrm{E}-08$ & 8.0672 & Up & S100 calcium binding protein $\mathrm{A} 10$ \\
\hline ILMN_1689725 & RPLP1 & 3.280536 & $5.45 \mathrm{E}-10$ & $2.42 \mathrm{E}-08$ & 8.061892 & Up & ribosomal protein lateral stalk subunit P1 \\
\hline ILMN_1756860 & TXNL1 & 2.503933 & $5.65 \mathrm{E}-10$ & $2.5 \mathrm{E}-08$ & 8.050325 & Up & thioredoxin like 1 \\
\hline ILMN_1719759 & TNC & 3.314571 & $5.77 \mathrm{E}-10$ & $2.54 \mathrm{E}-08$ & 8.04365 & Up & tenascin C \\
\hline ILMN_2318638 & TGIF1 & 2.07494 & $6.03 E-10$ & $2.63 \mathrm{E}-08$ & 8.029551 & Up & TGFB induced factor homeobox 1 \\
\hline ILMN_2400613 & SNX7 & 1.893439 & $6.11 \mathrm{E}-10$ & $2.66 \mathrm{E}-08$ & 8.025399 & Up & sorting nexin 7 \\
\hline ILMN_2104356 & COL1A2 & 3.560675 & $6.13 \mathrm{E}-10$ & $2.67 \mathrm{E}-08$ & 8.024708 & Up & collagen type I alpha 2 chain \\
\hline ILMN_2367215 & PRCP & 1.946656 & $6.16 \mathrm{E}-10$ & $2.68 \mathrm{E}-08$ & 8.022702 & Up & prolylcarboxypeptidase \\
\hline ILMN_1783852 & CD164 & 1.973328 & $6.44 \mathrm{E}-10$ & $2.78 \mathrm{E}-08$ & 8.008778 & Up & CD164 molecule \\
\hline ILMN_2181241 & RPL23AP64 & 2.528243 & $6.49 \mathrm{E}-10$ & $2.79 \mathrm{E}-08$ & 8.006577 & Up & ribosomal protein L23a pseudogene 64 \\
\hline ILMN_1801939 & CCNB2 & 3.013233 & $6.55 \mathrm{E}-10$ & $2.81 \mathrm{E}-08$ & 8.003633 & Up & cyclin B2 \\
\hline ILMN_1738150 & SUMO2 & 2.722215 & $6.63 \mathrm{E}-10$ & $2.84 \mathrm{E}-08$ & 7.999791 & Up & small ubiquitin like modifier 2 \\
\hline ILMN_3241234 & S100A11 & 2.932029 & $7.25 \mathrm{E}-10$ & $3.05 \mathrm{E}-08$ & 7.971319 & Up & S100 calcium binding protein A11 \\
\hline ILMN_1723978 & LGALS1 & 2.050485 & $7.37 \mathrm{E}-10$ & $3.09 \mathrm{E}-08$ & 7.966356 & Up & galectin 1 \\
\hline ILMN_3191695 & RAN & 2.043508 & 7.37E-10 & 3.09E-08 & 7.966308 & Up & RAN, member RAS oncogene family \\
\hline ILMN_2143795 & CYTOR & 2.354554 & $7.42 \mathrm{E}-10$ & $3.1 \mathrm{E}-08$ & 7.96432 & Up & cytoskeleton regulator RNA \\
\hline ILMN_1679587 & AP001086.1 & 2.194292 & $7.52 \mathrm{E}-10$ & 3.13E-08 & 7.959845 & Up & ribosomal protein L36a-like (RPL36AL) pseudogene \\
\hline ILMN_2169839 & CNBP & 2.01927 & $8.27 \mathrm{E}-10$ & 3.39E-08 & 7.929959 & Up & $\mathrm{CCHC}$-type zinc finger nucleic acid binding protein \\
\hline ILMN_3282587 & H3F3AP6 & 2.710797 & $8.56 \mathrm{E}-10$ & $3.49 \mathrm{E}-08$ & 7.919316 & Up & H3 histone, family $3 \mathrm{~A}$, pseudogene 6 \\
\hline ILMN_3241834 & RPL7 & 2.536782 & $9.03 \mathrm{E}-10$ & $3.63 \mathrm{E}-08$ & 7.902554 & Up & ribosomal protein $\mathrm{L} 7$ \\
\hline ILMN_1704385 & $\# \mathrm{~N} / \mathrm{A}$ & 2.528467 & $9.07 \mathrm{E}-10$ & $3.65 \mathrm{E}-08$ & 7.901037 & Up & NA \\
\hline ILMN_1721035 & MS4A6A & 2.690706 & $9.23 \mathrm{E}-10$ & $3.71 \mathrm{E}-08$ & 7.895351 & Up & membrane spanning 4-domains $A 6 A$ \\
\hline
\end{tabular}


medRxiv preprint doi: https://doi.org/10.1101/2020.12.21.20248616; this version posted December 23, 2020. The copyright holder for this preprint (which was not certified by peer review) is the author/funder, who has granted medRxiv a license to display the preprint in perpetuity.

All rights reserved. No reuse allowed without permission.

\begin{tabular}{|c|c|c|c|c|c|c|c|}
\hline ILMN_3227529 & RPS13 & 2.32539 & $9.27 \mathrm{E}-10$ & $3.72 \mathrm{E}-08$ & 7.894205 & Up & ribosomal protein $\mathrm{S} 13$ \\
\hline ILMN_1712545 & S100A3 & 2.089214 & $9.49 \mathrm{E}-10$ & $3.8 \mathrm{E}-08$ & 7.886634 & Up & S100 calcium binding protein A3 \\
\hline ILMN_3289352 & RPL6 & 2.973175 & $9.98 \mathrm{E}-10$ & $3.93 \mathrm{E}-08$ & 7.870991 & Up & ribosomal protein $\mathrm{L} 6$ \\
\hline ILMN_2214790 & LAMB1 & 2.207324 & $1.01 \mathrm{E}-09$ & $3.96 \mathrm{E}-08$ & 7.867438 & Up & laminin subunit beta 1 \\
\hline ILMN_3280020 & AC092128.1 & 2.209066 & $1.05 \mathrm{E}-09$ & $4.08 \mathrm{E}-08$ & 7.856139 & Up & ribosomal protein SA pseudogene 56 \\
\hline ILMN_2364022 & SLC16A3 & 2.114886 & $1.08 \mathrm{E}-09$ & 4.17E-08 & 7.845921 & Up & solute carrier family 16 member 3 \\
\hline ILMN_1733453 & RHOQ & 1.907755 & $1.08 \mathrm{E}-09$ & $4.17 \mathrm{E}-08$ & 7.845318 & Up & ras homolog family member $Q$ \\
\hline ILMN_1808837 & RPL7A & 1.962108 & $1.15 \mathrm{E}-09$ & $4.4 \mathrm{E}-08$ & 7.826315 & Up & ribosomal protein $\mathrm{L7a}$ \\
\hline ILMN_1680955 & AURKA & 2.1176 & $1.18 \mathrm{E}-09$ & $4.48 \mathrm{E}-08$ & 7.819309 & Up & aurora kinase $\mathrm{A}$ \\
\hline ILMN_2184184 & ANXA1 & 2.459557 & $1.18 \mathrm{E}-09$ & $4.5 \mathrm{E}-08$ & 7.817389 & Up & annexin $\mathrm{A} 1$ \\
\hline ILMN_3279712 & SMS & 2.112153 & $1.2 \mathrm{E}-09$ & $4.54 \mathrm{E}-08$ & 7.813821 & Up & spermine synthase \\
\hline ILMN_1695658 & KIF2OA & 2.296238 & $1.2 \mathrm{E}-09$ & 4.56E-08 & 7.812093 & Up & kinesin family member $20 \mathrm{~A}$ \\
\hline ILMN_2409220 & HMMR & 2.050473 & $1.22 \mathrm{E}-09$ & $4.61 \mathrm{E}-08$ & 7.807493 & Up & hyaluronan mediated motility receptor \\
\hline ILMN_3258321 & SNRPG & 2.093324 & $1.28 \mathrm{E}-09$ & 4.77E-08 & 7.792387 & Up & small nuclear ribonucleoprotein polypeptide $G$ \\
\hline ILMN_2294762 & AMY1A & 2.664914 & $1.35 \mathrm{E}-09$ & 4.97E-08 & 7.777221 & Up & amylase alpha $1 \mathrm{~A}$ (salivary) \\
\hline ILMN_1711899 & ANXA2 & 2.088768 & $1.36 \mathrm{E}-09$ & $5.02 \mathrm{E}-08$ & 7.773255 & Up & annexin $\mathrm{A} 2$ \\
\hline ILMN_1718984 & FCGBP & 2.695759 & $1.44 \mathrm{E}-09$ & $5.28 \mathrm{E}-08$ & 7.75492 & Up & Fc fragment of IgG binding protein \\
\hline ILMN_1740466 & TENT5A & 2.238462 & $1.45 \mathrm{E}-09$ & $5.28 \mathrm{E}-08$ & 7.754275 & Up & terminal nucleotidyltransferase $5 \mathrm{~A}$ \\
\hline ILMN_1709486 & SRPX & 2.942227 & $1.6 \mathrm{E}-09$ & 5.7E-08 & 7.723113 & Up & sushi repeat containing protein $\mathrm{X}$-linked \\
\hline ILMN_2359800 & MS4A6A & 2.474347 & $1.62 \mathrm{E}-09$ & $5.76 \mathrm{E}-08$ & 7.719308 & Up & membrane spanning 4-domains $A 6 A$ \\
\hline ILMN_2348788 & CD44 & 2.981206 & $1.65 \mathrm{E}-09$ & 5.86E-08 & 7.712785 & Up & CD44 molecule (Indian blood group) \\
\hline ILMN_3193623 & AC144530.1 & 2.64355 & $1.67 \mathrm{E}-09$ & 5.91E-08 & 7.709654 & Up & ribosomal protein L17 (RPL17) pseudogene \\
\hline ILMN_2046730 & S100A10 & 2.494961 & $1.76 \mathrm{E}-09$ & $6.17 \mathrm{E}-08$ & 7.693389 & Up & S100 calcium binding protein A10 \\
\hline ILMN_3282983 & HMGN1 & 2.191817 & $1.82 \mathrm{E}-09$ & $6.36 \mathrm{E}-08$ & 7.682735 & Up & high mobility group nucleosome binding domain 1 \\
\hline ILMN_1786139 & VKORC1 & 2.055262 & $1.86 \mathrm{E}-09$ & $6.48 \mathrm{E}-08$ & 7.676271 & Up & vitamin $\mathrm{K}$ epoxide reductase complex subunit 1 \\
\hline ILMN_2389844 & SP3 & 1.961499 & $1.88 \mathrm{E}-09$ & $6.53 \mathrm{E}-08$ & 7.672332 & Up & Sp3 transcription factor \\
\hline ILMN_2212909 & MELK & 3.081763 & $2.04 \mathrm{E}-09$ & $6.96 \mathrm{E}-08$ & 7.647452 & Up & $\begin{array}{l}\text { maternal embryonic leucine zipper kinase } \\
\text { family with sequence similarity } 115 \text {, member } \mathrm{C}\end{array}$ \\
\hline ILMN_3237627 & LOC154761 & 1.930237 & $2.12 \mathrm{E}-09$ & $7.21 \mathrm{E}-08$ & 7.634652 & Up & pseudogene \\
\hline ILMN_3195253 & RPL7 & 1.889471 & $2.13 \mathrm{E}-09$ & $7.22 \mathrm{E}-08$ & 7.633977 & Up & ribosomal protein L7 \\
\hline ILMN_1742813 & TMEM167A & 2.053367 & $2.36 \mathrm{E}-09$ & $7.85 \mathrm{E}-08$ & 7.60124 & Up & transmembrane protein $167 \mathrm{~A}$ \\
\hline ILMN_2370336 & MS4A4A & 2.600071 & $2.49 \mathrm{E}-09$ & $8.22 \mathrm{E}-08$ & 7.584493 & Up & membrane spanning 4-domains $\mathrm{A} 4 \mathrm{~A}$ \\
\hline ILMN_3201975 & PPIAP33 & 2.6673 & $2.56 \mathrm{E}-09$ & 8.39E-08 & 7.576518 & Up & peptidylprolylisomerase A pseudogene 33 \\
\hline ILMN_2410924 & PLOD2 & 2.763425 & $2.56 \mathrm{E}-09$ & $8.39 \mathrm{E}-08$ & 7.576506 & Up & procollagen-lysine, 2-oxoglutarate 5-dioxygenase 2 \\
\hline ILMN_3188076 & RPS7 & 2.301265 & $2.71 \mathrm{E}-09$ & $8.82 \mathrm{E}-08$ & 7.558353 & Up & ribosomal protein $\mathrm{S7}$ \\
\hline ILMN_1755173 & PLEKHA4 & 2.369135 & $2.75 \mathrm{E}-09$ & $8.92 \mathrm{E}-08$ & 7.554352 & Up & pleckstrin homology domain containing A4 \\
\hline ILMN_3293367 & RPS12 & 2.471475 & $2.96 \mathrm{E}-09$ & $9.51 \mathrm{E}-08$ & 7.530816 & Up & ribosomal protein $\mathrm{S} 12$ \\
\hline ILMN_2195914 & GGH & 2.394086 & 3.07E-09 & $9.79 \mathrm{E}-08$ & 7.519415 & Up & $\begin{array}{l}\text { gamma-glutamyl hydrolase } \\
\text { ADAM metallopeptidase with thrombospondin type }\end{array}$ \\
\hline ILMN_1805543 & ADAMTS9 & 1.959901 & 3.14E-09 & $1 \mathrm{E}-07$ & 7.512429 & Up & 1 motif 9 \\
\hline ILMN_1719749 & PTGES3 & 2.572848 & 3.15E-09 & $1 \mathrm{E}-07$ & 7.511313 & Up & prostaglandin E synthase 3 \\
\hline ILMN_2347888 & LARP4 & 1.898784 & 3.19E-09 & $1.01 \mathrm{E}-07$ & 7.50773 & Up & La ribonucleoprotein 4 \\
\hline ILMN_1720114 & GMNN & 2.212994 & $3.42 \mathrm{E}-09$ & $1.08 \mathrm{E}-07$ & 7.485715 & Up & geminin DNA replication inhibitor \\
\hline 1756326 & CKS & 2.153815 & 65 & $1.13 \mathrm{E}-\mathrm{C}$ & 7.46583 & Up & tein kinas \\
\hline
\end{tabular}


medRxiv preprint doi: https://doi.org/10.1101/2020.12.21.20248616; this version posted December 23, 2020. The copyright holder for this preprint (which was not certified by peer review) is the author/funder, who has granted medRxiv a license to display the preprint in perpetuity.

All rights reserved. No reuse allowed without permission.

\begin{tabular}{|c|c|c|c|c|c|c|c|}
\hline ILMN_2383349 & STEAP3 & 2.699724 & $3.96 \mathrm{E}-09$ & $1.22 \mathrm{E}-07$ & 7.440644 & Up & STEAP3 metalloreductase \\
\hline ILMN_2183885 & MPLKIP & 1.89151 & $4 \mathrm{E}-09$ & $1.23 \mathrm{E}-07$ & 7.437484 & Up & M-phase specific PLK1 interacting protein \\
\hline ILMN_1760089 & RPS2 & 2.167901 & $4.03 \mathrm{E}-09$ & $1.24 \mathrm{E}-07$ & 7.43479 & Up & ribosomal protein $S 2$ \\
\hline ILMN_3210538 & RPS10 & 2.106972 & $4.04 \mathrm{E}-09$ & $1.24 \mathrm{E}-07$ & 7.434003 & Up & ribosomal protein $\$ 10$ \\
\hline ILMN_2055700 & SLBP & 2.324086 & $4.18 \mathrm{E}-09$ & $1.28 \mathrm{E}-07$ & 7.423943 & Up & stem-loop binding protein \\
\hline ILMN_1751444 & NCAPG & 2.883496 & 4.19E-09 & $1.28 \mathrm{E}-07$ & 7.422977 & Up & non-SMC condensin I complex subunit $G$ \\
\hline ILMN_1661346 & HNRNPA1 & 1.919685 & $4.22 \mathrm{E}-09$ & $1.29 \mathrm{E}-07$ & 7.420508 & Up & heterogeneous nuclear ribonucleoprotein A1 \\
\hline ILMN_1679045 & SBDS & 2.279709 & 4.33E-09 & $1.31 \mathrm{E}-07$ & 7.412872 & Up & SBDS ribosome maturation factor \\
\hline ILMN_3178252 & RPS29 & 2.597136 & $4.56 \mathrm{E}-09$ & $1.37 \mathrm{E}-07$ & 7.396386 & Up & ribosomal protein $\$ 29$ \\
\hline ILMN_1767665 & GPX8 & 2.67501 & $4.65 \mathrm{E}-09$ & $1.4 \mathrm{E}-07$ & 7.390504 & Up & glutathione peroxidase 8 (putative) \\
\hline ILMN_3307868 & CHI3L1 & 4.20867 & $4.74 \mathrm{E}-09$ & $1.41 \mathrm{E}-07$ & 7.384308 & Up & chitinase 3 like 1 \\
\hline ILMN_1753196 & PTTG1 & 2.028525 & $4.9 \mathrm{E}-09$ & 1.45E-07 & 7.374021 & Up & $\begin{array}{c}\text { PTTG1 regulator of sister chromatid separation, } \\
\text { securin }\end{array}$ \\
\hline ILMN_1745607 & $\mathrm{A} 2 \mathrm{M}$ & 2.146484 & $5 \mathrm{E}-09$ & $1.47 \mathrm{E}-07$ & 7.368103 & Up & $\begin{array}{l}\quad \text { alpha-2-macroglobulin } \\
\text { major histocompatibility complex, class I, H }\end{array}$ \\
\hline ILMN_2130441 & $H L A-H$ & 2.308567 & $5.01 \mathrm{E}-09$ & $1.47 \mathrm{E}-07$ & 7.367149 & Up & (pseudogene) \\
\hline ILMN_3208715 & PPIAP43 & 3.353351 & $5.09 \mathrm{E}-09$ & $1.49 \mathrm{E}-07$ & 7.362668 & Up & peptidylprolylisomerase A pseudogene 43 \\
\hline ILMN_1726460 & RPL14 & 2.68186 & $5.16 \mathrm{E}-09$ & $1.51 \mathrm{E}-07$ & 7.358148 & Up & ribosomal protein L14 \\
\hline ILMN_1695880 & LOX & 3.386338 & $5.23 \mathrm{E}-09$ & $1.52 \mathrm{E}-07$ & 7.354121 & Up & lysyl oxidase \\
\hline ILMN_1679025 & RPS3A & 2.347938 & $5.48 \mathrm{E}-09$ & $1.58 \mathrm{E}-07$ & 7.339552 & Up & ribosomal protein S3A \\
\hline ILMN_1697268 & EMILIN2 & 1.913039 & $5.6 \mathrm{E}-09$ & $1.61 \mathrm{E}-07$ & 7.332859 & Up & elastin microfibrilinterfacer 2 \\
\hline ILMN_3235013 & RPS28 & 1.969933 & 5.74E-09 & $1.64 \mathrm{E}-07$ & 7.325172 & Up & $\begin{array}{c}\text { ribosomal protein } \mathrm{S} 28 \\
\text { methylenetetrahydrofolate dehydrogenase (NADP+ } \\
\text { dependent) } 2 \\
\text { methenyltetrahydrofolatecyclohydrolase }\end{array}$ \\
\hline ILMN_3290298 & PPIA & 2.387093 & $6.29 \mathrm{E}-09$ & $1.77 \mathrm{E}-07$ & 7.296907 & Up & peptidylprolylisomerase A \\
\hline ILMN_1717173 & ECT2 & 2.339012 & $6.73 \mathrm{E}-09$ & $1.88 \mathrm{E}-07$ & 7.275772 & Up & epithelial cell transforming 2 \\
\hline ILMN_1691053 & RPS2 & 1.982788 & $6.91 \mathrm{E}-09$ & $1.91 \mathrm{E}-07$ & 7.26795 & Up & ribosomal protein S2 \\
\hline ILMN_1673673 & PBK & 3.151525 & 7.14E-09 & $1.96 \mathrm{E}-07$ & 7.257546 & Up & $\begin{array}{c}\text { PDZ binding kinase } \\
\text { zinc finger and BTB domain containing } 8 \text { opposite }\end{array}$ \\
\hline ILMN_1802906 & ZBTB8OS & 1.89366 & $7.16 \mathrm{E}-09$ & $1.97 \mathrm{E}-07$ & 7.256608 & Up & strand \\
\hline ILMN_1795243 & RPS4X & 1.907108 & $7.33 \mathrm{E}-09$ & $2.01 \mathrm{E}-07$ & 7.249653 & Up & ribosomal protein $\$ 4$ X-linked \\
\hline ILMN_1788955 & PDLIM1 & 2.169014 & $7.56 \mathrm{E}-09$ & $2.06 \mathrm{E}-07$ & 7.239924 & Up & PDZ and LIM domain 1 \\
\hline ILMN_3286813 & AL109936.1 & 2.004298 & 7.59E-09 & $2.06 \mathrm{E}-07$ & 7.238879 & Up & ribosomal protein L29 (RPL29) pseudogene \\
\hline ILMN_3294074 & RPS12 & 2.153461 & 8.07E-09 & 2.17E-07 & 7.219744 & Up & ribosomal protein $\mathrm{S} 12$ \\
\hline ILMN_3301065 & RPS27A & 1.975769 & $8.84 \mathrm{E}-09$ & $2.35 \mathrm{E}-07$ & 7.19152 & Up & ribosomal protein $\$ 27 a$ \\
\hline ILMN_1759954 & PTMA & 2.842993 & $8.88 \mathrm{E}-09$ & $2.36 \mathrm{E}-07$ & 7.190218 & Up & prothymosin alpha \\
\hline ILMN_2056032 & CD99 & 2.245917 & $9.19 \mathrm{E}-09$ & $2.42 \mathrm{E}-07$ & 7.179487 & Up & CD99 molecule (Xg blood group) \\
\hline ILMN_2063584 & CLIC4 & 2.054374 & $9.73 \mathrm{E}-09$ & $2.54 \mathrm{E}-07$ & 7.161879 & Up & chloride intracellular channel 4 \\
\hline ILMN_1656670 & HLA-G & 2.191409 & $1 \mathrm{E}-08$ & $2.6 \mathrm{E}-07$ & 7.152736 & Up & major histocompatibility complex, class I, G \\
\hline ILMN_1655710 & RPS25 & 2.361426 & $1.01 \mathrm{E}-08$ & $2.61 \mathrm{E}-07$ & 7.151545 & Up & ribosomal protein $\$ 25$ \\
\hline ILMN_2138765 & PLIN2 & 2.602227 & $1.03 \mathrm{E}-08$ & $2.65 \mathrm{E}-07$ & 7.145525 & Up & perilipin 2 \\
\hline ILMN_1795474 & ССТ8 & 1.950474 & $1.03 E-08$ & $2.66 \mathrm{E}-07$ & 7.143366 & Up & chaperonin containing TCP1 subunit 8 \\
\hline ILMN_1665823 & TPT1 & 2.380391 & $1.05 \mathrm{E}-08$ & 2.7E-07 & 7.137506 & Up & tumor protein, translationally-controlled 1 \\
\hline ILMN_2086095 & ID2 & 2.029594 & $1.07 \mathrm{E}-08$ & $2.73 \mathrm{E}-07$ & 7.133574 & Up & inhibitor of DNA binding 2 \\
\hline ILMN_3261938 & TMSB10 & 1.9544 & $1.07 \mathrm{E}-08$ & $2.73 \mathrm{E}-07$ & 7.133404 & Up & thymosin beta 10 \\
\hline ILMN_1677814 & ABCC3 & 3.747183 & $1.1 \mathrm{E}-08$ & $2.79 \mathrm{E}-07$ & 7.124214 & Up & ATP binding cassette subfamily $C$ member 3 \\
\hline
\end{tabular}


medRxiv preprint doi: https://doi.org/10.1101/2020.12.21.20248616; this version posted December 23, 2020. The copyright holder for this preprint (which was not certified by peer review) is the author/funder, who has granted medRxiv a license to display the preprint in perpetuity. All rights reserved. No reuse allowed without permission.

\begin{tabular}{|c|c|c|c|c|c|c|c|}
\hline ILMN_1720998 & CA12 & 3.265552 & $1.14 \mathrm{E}-08$ & $2.87 \mathrm{E}-07$ & 7.11321 & Up & carbonic anhydrase 12 \\
\hline ILMN_2202948 & BUB1 & 2.302142 & $1.16 \mathrm{E}-08$ & $2.9 \mathrm{E}-07$ & 7.108878 & Up & BUB1 mitotic checkpoint serine/threonine kinase \\
\hline ILMN_1676213 & SRPX2 & 2.81698 & $1.16 \mathrm{E}-08$ & $2.9 \mathrm{E}-07$ & 7.108617 & Up & sushi repeat containing protein $X$-linked 2 \\
\hline ILMN_3259146 & BST2 & 2.421587 & $1.16 \mathrm{E}-08$ & $2.9 \mathrm{E}-07$ & 7.107746 & Up & bone marrow stromal cell antigen 2 \\
\hline ILMN_3242120 & RAP1B & 2.043785 & $1.2 \mathrm{E}-08$ & $2.98 \mathrm{E}-07$ & 7.097662 & Up & RAP1B, member of RAS oncogene family \\
\hline ILMN_2094587 & USP8 & 1.892987 & $1.2 \mathrm{E}-08$ & $2.98 \mathrm{E}-07$ & 7.097247 & Up & ubiquitin specific peptidase 8 \\
\hline ILMN_1670238 & CDC45 & 2.797454 & $1.21 \mathrm{E}-08$ & $3.01 \mathrm{E}-07$ & 7.093688 & Up & $\begin{array}{l}\text { cell division cycle } 45 \\
\text { neural precursor cell expressed, developmentally }\end{array}$ \\
\hline ILMN_2261076 & NEDD9 & 2.082582 & $1.22 \mathrm{E}-08$ & $3.02 \mathrm{E}-07$ & 7.092774 & Up & down-regulated 9 \\
\hline ILMN_1770922 & TMEM45A & 1.995366 & $1.23 \mathrm{E}-08$ & $3.04 \mathrm{E}-07$ & 7.089653 & Up & transmembrane protein $45 \mathrm{~A}$ \\
\hline ILMN_1803647 & FAM162A & 2.199196 & $1.28 \mathrm{E}-08$ & $3.14 \mathrm{E}-07$ & 7.078563 & Up & family with sequence similarity 162 member $A$ \\
\hline ILMN_3219455 & HSPD1 & 2.138162 & $1.28 \mathrm{E}-08$ & $3.15 \mathrm{E}-07$ & 7.077993 & Up & heat shock protein family $\mathrm{D}(\mathrm{Hsp} 60)$ member 1 \\
\hline ILMN_3238782 & RPS12 & 2.021448 & $1.37 \mathrm{E}-08$ & $3.34 \mathrm{E}-07$ & 7.056966 & Up & ribosomal protein $\mathrm{S} 12$ \\
\hline ILMN_1765446 & EMP3 & 2.381122 & $1.39 \mathrm{E}-08$ & $3.39 \mathrm{E}-07$ & 7.05128 & Up & epithelial membrane protein 3 \\
\hline ILMN_2326273 & CHI3L2 & 4.001245 & $1.39 \mathrm{E}-08$ & $3.39 \mathrm{E}-07$ & 7.051222 & Up & chitinase 3 like 2 \\
\hline ILMN_3264073 & RPS27 & 2.238851 & $1.51 \mathrm{E}-08$ & $3.63 \mathrm{E}-07$ & 7.027289 & Up & ribosomal protein $S 27$ \\
\hline ILMN_3214256 & PPIAP35 & 2.37574 & $1.52 \mathrm{E}-08$ & $3.65 \mathrm{E}-07$ & 7.02509 & Up & peptidylprolylisomerase A pseudogene 35 \\
\hline ILMN_2406501 & SOD2 & 2.894287 & $1.54 \mathrm{E}-08$ & 3.7E-07 & 7.020104 & Up & superoxide dismutase 2 \\
\hline ILMN_3283449 & RPS3 & 2.41434 & $1.57 \mathrm{E}-08$ & $3.76 \mathrm{E}-07$ & 7.014333 & Up & ribosomal protein $\mathrm{S} 3$ \\
\hline ILMN_3227315 & FTH1 & 2.999082 & $1.58 \mathrm{E}-08$ & $3.78 \mathrm{E}-07$ & 7.011696 & Up & ferritin heavy chain 1 \\
\hline ILMN_1797776 & PRSS23 & 2.018382 & $1.62 \mathrm{E}-08$ & $3.87 \mathrm{E}-07$ & 7.003983 & Up & serine protease 23 \\
\hline ILMN_1714335 & $\mathrm{RDH} 10$ & 2.361732 & $1.72 \mathrm{E}-08$ & 4.05E-07 & 6.986148 & Up & retinol dehydrogenase 10 \\
\hline ILMN_2308950 & AKAP12 & 2.213022 & $1.75 \mathrm{E}-08$ & $4.1 \mathrm{E}-07$ & 6.980816 & Up & A-kinase anchoring protein 12 \\
\hline ILMN_1746525 & FTH1 & 2.071555 & $1.79 \mathrm{E}-08$ & $4.18 \mathrm{E}-07$ & 6.974267 & Up & $\begin{array}{c}\text { ferritin heavy chain } 1 \\
\text { methylenetetrahydrofolate dehydrogenase (NADP+ } \\
\text { dependent) } 2\end{array}$ \\
\hline ILMN_2405521 & MTHFD2 & 1.925427 & $1.81 \mathrm{E}-08$ & $4.21 \mathrm{E}-07$ & 6.971596 & Up & $\begin{array}{l}\text { methenyltetrahydrofolatecyclohydrolase } \\
\text { neutral sphingomyelinase activation associated }\end{array}$ \\
\hline ILMN_3242288 & NSMAF & 2.728768 & $1.86 \mathrm{E}-08$ & $4.31 \mathrm{E}-07$ & 6.962346 & Up & factor $\hat{A}$ \\
\hline ILMN_3225121 & RPL23A & 1.988913 & $1.87 \mathrm{E}-08$ & $4.32 \mathrm{E}-07$ & 6.961179 & Up & ribosomal protein L23a \\
\hline ILMN_1737184 & CDCA7 & 2.29942 & $1.87 \mathrm{E}-08$ & $4.33 \mathrm{E}-07$ & 6.960611 & Up & cell division cycle associated 7 \\
\hline ILMN_3231881 & PTMA & 2.367641 & $1.96 \mathrm{E}-08$ & $4.49 \mathrm{E}-07$ & 6.946151 & Up & prothymosin alpha \\
\hline ILMN_3245517 & RPS29 & 1.999784 & $2 \mathrm{E}-08$ & 4.56E-07 & 6.940183 & Up & ribosomal protein $\mathrm{S} 29$ \\
\hline ILMN_1730628 & RNASE2 & 1.947205 & $2.09 \mathrm{E}-08$ & $4.73 \mathrm{E}-07$ & 6.926696 & Up & ribonuclease A family member 2 \\
\hline ILMN_1724533 & LY96 & 2.002801 & $2.1 \mathrm{E}-08$ & $4.75 \mathrm{E}-07$ & 6.925289 & Up & lymphocyte antigen 96 \\
\hline ILMN_1721868 & KPNA2 & 1.929179 & $2.14 \mathrm{E}-08$ & $4.81 \mathrm{E}-07$ & 6.919583 & Up & karyopherin subunit alpha 2 \\
\hline ILMN_1753111 & NAMPT & 2.191403 & $2.17 E-08$ & $4.88 \mathrm{E}-07$ & 6.914919 & Up & nicotinamidephosphoribosyltransferase \\
\hline ILMN_1739496 & PRRX1 & 1.998681 & $2.17 \mathrm{E}-08$ & $4.88 \mathrm{E}-07$ & 6.914611 & Up & paired related homeobox 1 \\
\hline ILMN_2285996 & PCLAF & 2.563175 & $2.23 \mathrm{E}-08$ & $5 \mathrm{E}-07$ & 6.906251 & Up & PCNA clamp associated factor \\
\hline ILMN_2196984 & OIP5 & 2.120753 & $2.31 \mathrm{E}-08$ & $5.14 \mathrm{E}-07$ & 6.896196 & Up & Opa interacting protein 5 \\
\hline ILMN_1750278 & FTH1 & 1.982402 & $2.55 \mathrm{E}-08$ & $5.59 \mathrm{E}-07$ & 6.865882 & Up & ferritin heavy chain 1 \\
\hline ILMN_1789007 & APOC1 & 2.150314 & $2.64 \mathrm{E}-08$ & $5.73 \mathrm{E}-07$ & 6.85528 & Up & apolipoprotein C1 \\
\hline ILMN_1768582 & PPP2CB & 2.463216 & $2.7 \mathrm{E}-08$ & $5.85 \mathrm{E}-07$ & 6.847459 & Up & protein phosphatase 2 catalytic subunit beta \\
\hline ILMN_3239771 & DLGAP5 & 2.60617 & $2.72 \mathrm{E}-08$ & $5.89 \mathrm{E}-07$ & 6.845304 & Up & DLG associated protein 5 \\
\hline ILMN_2045419 & BNIP3L & 2.088942 & $2.76 \mathrm{E}-08$ & $5.95 \mathrm{E}-07$ & 6.841524 & Up & $\mathrm{BCL2}$ interacting protein 3 like \\
\hline ILMN_2092536 & HSPE1 & 1.902107 & $2.81 \mathrm{E}-08$ & $6.04 \mathrm{E}-07$ & 6.83554 & Up & heat shock protein family $\mathrm{E}$ (Hsp10) member 1 \\
\hline
\end{tabular}


medRxiv preprint doi: https://doi.org/10.1101/2020.12.21.20248616; this version posted December 23, 2020. The copyright holder for this preprint (which was not certified by peer review) is the author/funder, who has granted medRxiv a license to display the preprint in perpetuity. All rights reserved. No reuse allowed without permission.

\begin{tabular}{|c|c|c|c|c|c|c|c|}
\hline ILMN_3199974 & RPS27 & 2.203135 & $2.83 \mathrm{E}-08$ & $6.08 \mathrm{E}-07$ & 6.833197 & Up & ribosomal protein $\$ 27$ \\
\hline ILMN_1668861 & TPI1 & 2.134795 & $3 \mathrm{E}-08$ & $6.37 \mathrm{E}-07$ & 6.815302 & Up & triosephosphateisomerase 1 \\
\hline ILMN_3200539 & AL158823.1 & 2.098219 & $3.03 \mathrm{E}-08$ & $6.43 \mathrm{E}-07$ & 6.812244 & Up & ribosomal protein L21 (RPL21) pseudogene \\
\hline ILMN_1763907 & CENPW & 2.024119 & $3.14 \mathrm{E}-08$ & $6.64 \mathrm{E}-07$ & 6.801487 & Up & centromere protein $W$ \\
\hline ILMN_2064725 & METTL7B & 2.729173 & $3.19 \mathrm{E}-08$ & $6.72 \mathrm{E}-07$ & 6.796793 & Up & methyltransferase like 7B \\
\hline ILMN_3230435 & VOPP1 & 3.027049 & $3.2 \mathrm{E}-08$ & $6.75 \mathrm{E}-07$ & 6.795504 & Up & VOPP1 WW domain binding protein \\
\hline ILMN_1764090 & AK4 & 1.88741 & $3.3 \mathrm{E}-08$ & $6.92 \mathrm{E}-07$ & 6.786386 & Up & adenylate kinase 4 \\
\hline ILMN_1652003 & GNG10 & 1.881122 & $3.31 \mathrm{E}-08$ & $6.93 \mathrm{E}-07$ & 6.785627 & Up & G protein subunit gamma 10 \\
\hline ILMN_1807277 & IFI30 & 2.524538 & $3.51 \mathrm{E}-08$ & $7.25 \mathrm{E}-07$ & 6.76749 & Up & IFI30 lysosomalthiolreductase \\
\hline ILMN_1659405 & RPL31 & 1.975558 & $3.52 \mathrm{E}-08$ & $7.27 \mathrm{E}-07$ & 6.766636 & Up & $\begin{array}{c}\text { ribosomal protein L31 } \\
\text { LSM5 homolog, U6 small nuclear RNA and mRNA }\end{array}$ \\
\hline ILMN_2116556 & LSM5 & 1.891337 & $3.65 \mathrm{E}-08$ & $7.48 \mathrm{E}-07$ & 6.755023 & Up & degradation associated \\
\hline ILMN_3211906 & EEF1B2 & 2.180265 & $3.66 \mathrm{E}-08$ & 7.49E-07 & 6.754572 & Up & eukaryotic translation elongation factor 1 beta 2 \\
\hline ILMN_1792138 & UQCRH & 1.941979 & $3.68 \mathrm{E}-08$ & $7.52 \mathrm{E}-07$ & 6.753206 & Up & ubiquinol-cytochrome $\mathrm{c}$ reductase hinge protein \\
\hline ILMN_1687978 & PHLDA1 & 2.128162 & $3.81 \mathrm{E}-08$ & $7.75 \mathrm{E}-07$ & 6.741944 & Up & pleckstrin homology like domain family A member 1 \\
\hline ILMN_2148785 & GBP1 & 2.068823 & $3.84 \mathrm{E}-08$ & $7.79 E-07$ & 6.739959 & Up & guanylate binding protein 1 \\
\hline ILMN_1758164 & STC1 & 2.091814 & 4.19E-08 & $8.38 \mathrm{E}-07$ & 6.713303 & Up & stanniocalcin 1 \\
\hline ILMN_1686884 & IL1RAP & 2.134839 & $4.29 \mathrm{E}-08$ & $8.55 \mathrm{E}-07$ & 6.705593 & Up & interleukin 1 receptor accessory protein \\
\hline ILMN_1748904 & WTAP & 2.103005 & $4.73 \mathrm{E}-08$ & $9.22 \mathrm{E}-07$ & 6.676185 & Up & WT1 associated protein \\
\hline ILMN_2349459 & BIRC5 & 2.385823 & $4.92 \mathrm{E}-08$ & $9.55 \mathrm{E}-07$ & 6.663701 & Up & baculoviral IAP repeat containing 5 \\
\hline ILMN_2382942 & CA12 & 2.381679 & 4.97E-08 & $9.63 \mathrm{E}-07$ & 6.660862 & Up & $\begin{array}{c}\text { carbonic anhydrase } 12 \\
\text { diazepam binding inhibitor, acyl-CoA binding }\end{array}$ \\
\hline ILMN_1755926 & DBI & 2.117418 & $4.98 \mathrm{E}-08$ & $9.64 \mathrm{E}-07$ & 6.660302 & Up & protein \\
\hline ILMN_1807423 & IGF2BP3 & 2.494894 & $5.11 \mathrm{E}-08$ & $9.87 \mathrm{E}-07$ & 6.6522 & Up & insulin like growth factor 2 mRNA binding protein 3 \\
\hline ILMN_1675448 & ZFP36L1 & 1.989362 & $5.42 \mathrm{E}-08$ & $1.04 \mathrm{E}-06$ & 6.634241 & Up & ZFP36 ring finger protein like 1 \\
\hline ILMN_2224103 & PAPSS1 & 1.943015 & $5.42 \mathrm{E}-08$ & $1.04 \mathrm{E}-06$ & 6.634184 & Up & 3'-phosphoadenosine 5'-phosphosulfate synthase 1 \\
\hline ILMN_1706013 & FTH1 & 1.886335 & $5.44 \mathrm{E}-08$ & $1.04 \mathrm{E}-06$ & 6.63302 & Up & ferritin heavy chain 1 \\
\hline ILMN_3305475 & TPI1 & 2.174888 & $5.5 \mathrm{E}-08$ & $1.05 \mathrm{E}-06$ & 6.630035 & Up & triosephosphateisomerase 1 \\
\hline ILMN_1725090 & CTHRC1 & 2.655415 & $5.6 \mathrm{E}-08$ & $1.06 \mathrm{E}-06$ & 6.624334 & Up & $\begin{array}{l}\text { collagen triple helix repeat containing } 1 \\
\text { protein phosphatase } 1 \text { regulatory inhibitor subunit }\end{array}$ \\
\hline ILMN_1659888 & PPP1R14B & 1.995379 & $5.74 \mathrm{E}-08$ & $1.09 \mathrm{E}-06$ & 6.616645 & Up & $14 \mathrm{~B}$ \\
\hline ILMN_2375879 & VEGFA & 3.027996 & $6.1 \mathrm{E}-08$ & $1.15 \mathrm{E}-06$ & 6.597887 & Up & vascular endothelial growth factor $A$ \\
\hline ILMN_1803799 & EIF4E & 1.97241 & $6.12 \mathrm{E}-08$ & $1.15 \mathrm{E}-06$ & 6.597269 & Up & eukaryotic translation initiation factor $4 \mathrm{E}$ \\
\hline ILMN_1764629 & SLC39A14 & 2.085857 & $6.13 \mathrm{E}-08$ & $1.15 \mathrm{E}-06$ & 6.596404 & Up & solute carrier family 39 member 14 \\
\hline ILMN_1814369 & C21orf62 & 2.461285 & $6.25 \mathrm{E}-08$ & $1.17 \mathrm{E}-06$ & 6.590658 & Up & chromosome 21 open reading frame 62 \\
\hline ILMN_1773262 & ESM1 & 1.899299 & $6.33 \mathrm{E}-08$ & $1.18 \mathrm{E}-06$ & 6.586621 & Up & endothelial cell specific molecule 1 \\
\hline ILMN_1815184 & ASPM & 2.552052 & $6.36 \mathrm{E}-08$ & 1.19E-06 & 6.585464 & Up & abnormal spindle microtubule assembly \\
\hline ILMN_3226875 & RPS27A & 1.998774 & $6.87 \mathrm{E}-08$ & $1.27 \mathrm{E}-06$ & 6.561839 & Up & ribosomal protein $\$ 27 a$ \\
\hline ILMN_1786125 & CCNA2 & 2.002342 & $6.95 \mathrm{E}-08$ & $1.28 \mathrm{E}-06$ & 6.558149 & Up & $\operatorname{cyclin} A 2$ \\
\hline ILMN_3217285 & HNRNPK & 1.887352 & $7.02 \mathrm{E}-08$ & $1.29 \mathrm{E}-06$ & 6.555292 & Up & heterogeneous nuclear ribonucleoprotein $\mathrm{K}$ \\
\hline ILMN_2409298 & NUSAP1 & 2.462982 & $7.31 \mathrm{E}-08$ & $1.34 \mathrm{E}-06$ & 6.542808 & Up & nucleolar and spindle associated protein 1 \\
\hline ILMN_2379599 & $\mathrm{CD} 163$ & 3.357749 & $7.52 \mathrm{E}-08$ & $1.37 \mathrm{E}-06$ & 6.534148 & Up & CD163 molecule \\
\hline ILMN_1677697 & RPS26P35 & 2.421801 & $7.59 \mathrm{E}-08$ & $1.38 \mathrm{E}-06$ & 6.531226 & Up & ribosomal protein S26 pseudogene 35 \\
\hline ILMN_1712803 & CCNB1 & 1.933311 & $8.69 \mathrm{E}-08$ & $1.55 \mathrm{E}-06$ & 6.489951 & Up & cyclin B1 \\
\hline ILMN_3285153 & RPS26 & 2.761623 & $8.87 \mathrm{E}-08$ & $1.58 \mathrm{E}-06$ & 6.483612 & Up & ribosomal protein $\$ 26$ \\
\hline
\end{tabular}


medRxiv preprint doi: https://doi.org/10.1101/2020.12.21.20248616; this version posted December 23, 2020. The copyright holder for this preprint (which was not certified by peer review) is the author/funder, who has granted medRxiv a license to display the preprint in perpetuity. All rights reserved. No reuse allowed without permission.

\begin{tabular}{|c|c|c|c|c|c|c|c|}
\hline ILMN_1805410 & C15orf48 & 2.128301 & $9.05 \mathrm{E}-08$ & $1.61 \mathrm{E}-06$ & 6.477301 & Up & chromosome 15 open reading frame 48 \\
\hline ILMN_1696911 & FTH1 & 2.418797 & $9.11 \mathrm{E}-08$ & $1.62 \mathrm{E}-06$ & 6.475295 & Up & ferritin heavy chain 1 \\
\hline ILMN_1660549 & WLS & 1.996875 & $9.3 \mathrm{E}-08$ & $1.64 \mathrm{E}-06$ & 6.469262 & Up & Wnt ligand secretion mediator \\
\hline ILMN_1792679 & ITGA5 & 1.915419 & $9.33 \mathrm{E}-08$ & $1.65 \mathrm{E}-06$ & 6.46828 & Up & integrin subunit alpha 5 \\
\hline ILMN_3216336 & TPT1 & 2.123158 & $9.66 \mathrm{E}-08$ & $1.7 \mathrm{E}-06$ & 6.457461 & Up & tumor protein, translationally-controlled 1 \\
\hline ILMN_1708934 & ADM & 2.596216 & $9.79 \mathrm{E}-08$ & $1.72 \mathrm{E}-06$ & 6.453328 & Up & adrenomedullin \\
\hline ILMN_1751776 & CKAP2L & 2.279748 & $1.03 \mathrm{E}-07$ & $1.79 \mathrm{E}-06$ & 6.437003 & Up & cytoskeleton associated protein 2 like \\
\hline ILMN_1786065 & UHRF1 & 2.221647 & $1.05 \mathrm{E}-07$ & $1.82 \mathrm{E}-06$ & 6.431922 & Up & ubiquitin like with PHD and ring finger domains 1 \\
\hline ILMN_2173835 & FTH1P3 & 2.170604 & $1.06 \mathrm{E}-07$ & $1.82 \mathrm{E}-06$ & 6.430484 & Up & ferritin heavy chain 1 pseudogene 3 \\
\hline ILMN_2201678 & FSTL1 & 1.969199 & $1.06 \mathrm{E}-07$ & $1.83 \mathrm{E}-06$ & 6.428582 & Up & follistatin like 1 \\
\hline ILMN_1801257 & CENPA & 1.90338 & $1.1 \mathrm{E}-07$ & $1.89 \mathrm{E}-06$ & 6.41799 & Up & $\begin{array}{l}\text { centromere protein } A \\
\text { heterogeneous nuclear ribonucleoprotein A1 }\end{array}$ \\
\hline ILMN_3297996 & HNRNPA1P53 & 2.148325 & $1.11 \mathrm{E}-07$ & $1.9 \mathrm{E}-06$ & 6.416178 & Up & pseudogene 53 \\
\hline ILMN_1729117 & COL5A2 & 2.129093 & $1.11 \mathrm{E}-07$ & $1.9 \mathrm{E}-06$ & 6.414711 & Up & collagen type $V$ alpha 2 chain \\
\hline ILMN_1703906 & HJURP & 2.071357 & $1.15 \mathrm{E}-07$ & $1.96 \mathrm{E}-06$ & 6.403208 & Up & Holliday junction recognition protein \\
\hline ILMN_2134538 & FTH1 & 2.443989 & $1.18 \mathrm{E}-07$ & $1.99 \mathrm{E}-06$ & 6.397323 & Up & ferritin heavy chain 1 \\
\hline ILMN_1732296 & ID3 & 1.983682 & $1.27 \mathrm{E}-07$ & 2.13E-06 & 6.373657 & Up & inhibitor of DNA binding 3 , HLH protein \\
\hline ILMN_1749829 & DLGAP5 & 2.276574 & $1.3 \mathrm{E}-07$ & $2.17 \mathrm{E}-06$ & 6.366961 & Up & DLG associated protein 5 \\
\hline ILMN_2051373 & NEK2 & 2.309143 & $1.45 \mathrm{E}-07$ & 2.37E-06 & 6.332967 & Up & NIMA related kinase 2 \\
\hline ILMN_1664718 & CYP51A1 & 1.948331 & $1.65 \mathrm{E}-07$ & $2.64 \mathrm{E}-06$ & 6.293751 & Up & cytochrome P450 family 51 subfamily A member 1 \\
\hline ILMN_1746888 & PCOLCE2 & 2.251156 & $1.73 \mathrm{E}-07$ & $2.74 \mathrm{E}-06$ & 6.279889 & Up & procollagen C-endopeptidase enhancer 2 \\
\hline ILMN_1696302 & FABP5 & 3.11089 & $1.77 \mathrm{E}-07$ & 2.79E-06 & 6.272644 & Up & fatty acid binding protein 5 \\
\hline ILMN_1659990 & HILPDA & 2.355975 & $1.83 \mathrm{E}-07$ & $2.87 \mathrm{E}-06$ & 6.262954 & Up & hypoxia inducible lipid droplet associated \\
\hline ILMN_2071809 & MGP & 2.744703 & $1.97 \mathrm{E}-07$ & $3.07 E-06$ & 6.239368 & Up & matrix Gla protein \\
\hline ILMN_1704154 & TNFRSF19 & 1.934978 & $1.99 \mathrm{E}-07$ & $3.09 \mathrm{E}-06$ & 6.237123 & Up & TNF receptor superfamily member 19 \\
\hline ILMN_1797731 & MS4A6A & 2.231349 & $2.12 \mathrm{E}-07$ & $3.24 \mathrm{E}-06$ & 6.218186 & Up & membrane spanning 4-domains $A 6 A$ \\
\hline ILMN_1801616 & EMP1 & 2.088735 & $2.27 \mathrm{E}-07$ & $3.46 \mathrm{E}-06$ & 6.195977 & Up & epithelial membrane protein 1 \\
\hline ILMN_2222008 & KIFC1 & 2.259846 & $2.32 \mathrm{E}-07$ & $3.52 \mathrm{E}-06$ & 6.189478 & Up & kinesin family member $\mathrm{C} 1$ \\
\hline ILMN_1710676 & FBXO5 & 1.979248 & $2.41 \mathrm{E}-07$ & $3.62 \mathrm{E}-06$ & 6.178254 & Up & F-box protein 5 \\
\hline ILMN_1803882 & VEGFA & 2.313095 & $2.49 \mathrm{E}-07$ & $3.73 \mathrm{E}-06$ & 6.168372 & Up & vascular endothelial growth factor $A$ \\
\hline ILMN_1777564 & MAD2L1 & 2.166484 & $2.61 \mathrm{E}-07$ & $3.88 \mathrm{E}-06$ & 6.154528 & Up & mitotic arrest deficient 2 like 1 \\
\hline ILMN_2380237 & C1OTNF1 & 2.337899 & $2.64 \mathrm{E}-07$ & $3.92 \mathrm{E}-06$ & 6.150566 & Up & $\mathrm{C} 1 \mathrm{q}$ and $\mathrm{TNF}$ related 1 \\
\hline ILMN_3205271 & HMGB1 & 2.207262 & $2.7 \mathrm{E}-07$ & $4 \mathrm{E}-06$ & 6.143206 & Up & high mobility group box 1 \\
\hline ILMN_1666305 & CDKN3 & 2.15736 & $2.71 \mathrm{E}-07$ & $4 \mathrm{E}-06$ & 6.142903 & Up & cyclin dependent kinase inhibitor 3 \\
\hline ILMN_2181060 & CKAP2 & 1.881238 & $2.8 \mathrm{E}-07$ & 4.12E-06 & 6.132389 & Up & cytoskeleton associated protein 2 \\
\hline ILMN_2223903 & PPIC & 2.13406 & $2.84 \mathrm{E}-07$ & 4.17E-06 & 6.128036 & Up & peptidylprolylisomerase C \\
\hline ILMN_3292163 & PPIAP47 & 1.909449 & $2.85 \mathrm{E}-07$ & 4.18E-06 & 6.126699 & Up & peptidylprolylisomerase A pseudogene 47 \\
\hline ILMN_1790529 & LUM & 2.749374 & $2.87 \mathrm{E}-07$ & $4.2 \mathrm{E}-06$ & 6.125297 & Up & lumican \\
\hline ILMN_2347592 & NMB & 2.452706 & $2.91 \mathrm{E}-07$ & 4.25E-06 & 6.120762 & Up & neuromedin B \\
\hline ILMN_1725139 & CA9 & 2.463889 & $3.04 \mathrm{E}-07$ & 4.42E-06 & 6.107264 & Up & carbonic anhydrase 9 \\
\hline ILMN_1777233 & E2F2 & 2.462294 & $3.11 \mathrm{E}-07$ & $4.5 \mathrm{E}-06$ & 6.100721 & Up & $\begin{array}{l}\text { E2F transcription factor } 2 \\
\text { protein phosphatase } 1 \text { regulatory inhibitor subunit }\end{array}$ \\
\hline ILMN_3299365 & PPP1R14B & 1.925455 & $3.39 \mathrm{E}-07$ & 4.87E-06 & 6.074113 & Up & $14 \mathrm{~B}$ \\
\hline ILMN_1796949 & TPX2 & 1.899637 & $3.6 \mathrm{E}-07$ & $5.12 \mathrm{E}-06$ & 6.056218 & Up & TPX2 microtubule nucleation factor \\
\hline
\end{tabular}


medRxiv preprint doi: https://doi.org/10.1101/2020.12.21.20248616; this version posted December 23, 2020. The copyright holder for this preprint (which was not certified by peer review) is the author/funder, who has granted medRxiv a license to display the preprint in perpetuity.

All rights reserved. No reuse allowed without permission.

\begin{tabular}{|c|c|c|c|c|c|c|c|}
\hline ILMN_3290100 & AL589647.1 & 1.950739 & $3.76 \mathrm{E}-07$ & $5.32 \mathrm{E}-06$ & 6.042431 & Up & ribosomal protein L21 (RPL21) pseudogene \\
\hline ILMN_2212878 & ESM1 & 2.367771 & $3.84 \mathrm{E}-07$ & 5.41E-06 & 6.03638 & Up & endothelial cell specific molecule 1 \\
\hline ILMN_3214532 & RPL21P28 & 2.393695 & $3.89 \mathrm{E}-07$ & $5.47 \mathrm{E}-06$ & 6.032476 & Up & $\begin{array}{l}\text { ribosomal protein } L 21 \text { pseudogene } 28 \\
\text { heterogeneous nuclear ribonucleoprotein A1 }\end{array}$ \\
\hline ILMN_3197767 & HNRNPA1P35 & 2.100474 & $4.2 \mathrm{E}-07$ & $5.83 \mathrm{E}-06$ & 6.008797 & Up & pseudogene 35 \\
\hline ILMN_1723678 & PRPH & 2.479257 & $4.5 \mathrm{E}-07$ & $6.17 \mathrm{E}-06$ & 5.988013 & Up & peripherin \\
\hline ILMN_1668863 & LYPD1 & 1.910459 & 4.63E-07 & $6.32 \mathrm{E}-06$ & 5.97909 & Up & LY6/PLAUR domain containing 1 \\
\hline ILMN_1654268 & HMGB2 & 1.909799 & 4.93E-07 & $6.66 \mathrm{E}-06$ & 5.959904 & Up & high mobility group box 2 \\
\hline ILMN_2145670 & TNC & 2.007948 & $6.6 \mathrm{E}-07$ & $8.55 \mathrm{E}-06$ & 5.870985 & Up & tenascin C \\
\hline ILMN_1683450 & CDCA5 & 2.070358 & $6.75 \mathrm{E}-07$ & $8.71 \mathrm{E}-06$ & 5.864133 & Up & cell division cycle associated 5 \\
\hline ILMN_1684217 & AURKB & 2.040535 & $7.18 \mathrm{E}-07$ & $9.19 \mathrm{E}-06$ & 5.845217 & Up & aurora kinase $B$ \\
\hline ILMN_1728934 & PRC1 & 1.994804 & $7.65 \mathrm{E}-07$ & $9.7 \mathrm{E}-06$ & 5.82558 & Up & protein regulator of cytokinesis 1 \\
\hline ILMN_1796316 & MMPg & 2.958285 & 7.69E-07 & $9.74 \mathrm{E}-06$ & 5.823956 & Up & matrix metallopeptidase 9 \\
\hline ILMN_2143155 & KIF11 & 1.939181 & 7.73E-07 & $9.78 \mathrm{E}-06$ & 5.822488 & Up & kinesin family member 11 \\
\hline ILMN_1746085 & IGFBP3 & 2.843429 & $7.84 \mathrm{E}-07$ & $9.9 \mathrm{E}-06$ & 5.818025 & Up & insulin like growth factor binding protein 3 \\
\hline ILMN_1733270 & CD163 & 2.749352 & $8.3 \mathrm{E}-07$ & $1.04 \mathrm{E}-05$ & 5.800678 & Up & CD163 molecule \\
\hline ILMN_3257030 & FTH1 & 1.904494 & $8.4 \mathrm{E}-07$ & $1.05 \mathrm{E}-05$ & 5.79713 & Up & ferritin heavy chain 1 \\
\hline ILMN_1779852 & FABP5P3 & 2.564603 & 8.67E-07 & $1.08 \mathrm{E}-05$ & 5.787492 & Up & fatty acid binding protein 5 pseudogene 3 \\
\hline ILMN_2117508 & CTHRC1 & 2.396771 & $8.71 \mathrm{E}-07$ & $1.08 \mathrm{E}-05$ & 5.78612 & Up & collagen triple helix repeat containing 1 \\
\hline ILMN_1738147 & NES & 2.093414 & $8.72 \mathrm{E}-07$ & $1.08 \mathrm{E}-05$ & 5.785557 & Up & nestin \\
\hline ILMN_1707124 & TFPI & 1.987902 & $8.91 \mathrm{E}-07$ & $1.1 \mathrm{E}-05$ & 5.778998 & Up & $\begin{array}{l}\text { tissue factor pathway inhibitor } \\
\text { major histocompatibility complex, class I, H }\end{array}$ \\
\hline ILMN_1666078 & HLA-H & 2.400374 & $9.22 \mathrm{E}-07$ & $1.14 \mathrm{E}-05$ & 5.768788 & Up & (pseudogene) \\
\hline ILMN_1801205 & GPNMB & 2.503419 & $9.45 \mathrm{E}-07$ & $1.16 \mathrm{E}-05$ & 5.761138 & Up & glycoprotein nmb \\
\hline ILMN_1722622 & CD163 & 3.152101 & $9.64 \mathrm{E}-07$ & $1.18 \mathrm{E}-05$ & 5.755195 & Up & CD163 molecule \\
\hline ILMN_1806165 & $\# \mathrm{~N} / \mathrm{A}$ & 2.502181 & $1 \mathrm{E}-06$ & $1.22 \mathrm{E}-05$ & 5.743962 & Up & NA \\
\hline ILMN_2203271 & FPR3 & 2.3159 & $1.05 \mathrm{E}-06$ & $1.27 \mathrm{E}-05$ & 5.728993 & Up & formyl peptide receptor 3 \\
\hline ILMN_1786444 & LPL & 2.415893 & $1.05 \mathrm{E}-06$ & $1.27 \mathrm{E}-05$ & 5.72877 & Up & lipoprotein lipase \\
\hline ILMN_1671800 & CRNDE & 1.946229 & $1.07 \mathrm{E}-06$ & $1.29 \mathrm{E}-05$ & 5.722648 & Up & colorectal neoplasia differentially expressed \\
\hline ILMN_1665865 & IGFBP4 & 1.889568 & $1.15 \mathrm{E}-06$ & $1.37 \mathrm{E}-05$ & 5.702418 & Up & insulin like growth factor binding protein 4 \\
\hline ILMN_1731184 & MELK & 1.952939 & $1.22 \mathrm{E}-06$ & $1.45 \mathrm{E}-05$ & 5.681929 & Up & maternal embryonic leucine zipper kinase \\
\hline ILMN_1682928 & CPVL & 2.04914 & $1.25 \mathrm{E}-06$ & $1.47 \mathrm{E}-05$ & 5.675897 & Up & carboxypeptidasevitellogenic like \\
\hline ILMN_1728972 & PIMREG & 2.198263 & $1.29 \mathrm{E}-06$ & $1.51 \mathrm{E}-05$ & 5.666145 & Up & PICALM interacting mitotic regulator \\
\hline ILMN_1727815 & $\mathrm{CFI}$ & 1.904234 & $1.3 \mathrm{E}-06$ & $1.53 \mathrm{E}-05$ & 5.663268 & Up & complement factor I \\
\hline ILMN_1800512 & HMOX1 & 2.632018 & $1.31 \mathrm{E}-06$ & $1.53 \mathrm{E}-05$ & 5.661075 & Up & hemeoxygenase 1 \\
\hline ILMN_1753613 & HOXA5 & 3.151581 & $1.42 \mathrm{E}-06$ & $1.64 \mathrm{E}-05$ & 5.637489 & Up & homeobox A5 \\
\hline ILMN_1737195 & CENPK & 1.905516 & $1.49 \mathrm{E}-06$ & $1.71 \mathrm{E}-05$ & 5.622309 & Up & centromere protein $\mathrm{K}$ \\
\hline ILMN_2396875 & IGFBP3 & 2.750338 & $1.55 \mathrm{E}-06$ & $1.77 \mathrm{E}-05$ & 5.610464 & Up & insulin like growth factor binding protein 3 \\
\hline ILMN_3178258 & FABP5P2 & 2.434412 & $1.56 \mathrm{E}-06$ & $1.77 \mathrm{E}-05$ & 5.608491 & Up & fatty acid binding protein 5 pseudogene 2 \\
\hline ILMN_1677906 & MIR1244-3 & 1.887256 & $1.57 \mathrm{E}-06$ & 1.79E-05 & 5.604873 & Up & microRNA $1244-3$ \\
\hline ILMN_1785272 & COL1A2 & 2.555215 & $1.71 \mathrm{E}-06$ & $1.93 \mathrm{E}-05$ & 5.579696 & Up & collagen type I alpha 2 chain \\
\hline ILMN_1736178 & AEBP1 & 1.925256 & $1.76 \mathrm{E}-06$ & $1.97 \mathrm{E}-05$ & 5.571236 & Up & AE binding protein 1 \\
\hline ILMN_1761833 & SLC4OA1 & 1.912536 & $1.76 \mathrm{E}-06$ & $1.98 \mathrm{E}-05$ & 5.570618 & Up & solute carrier family 40 member 1 \\
\hline ILMN_2202940 & CHPT1 & 1.880014 & $1.91 \mathrm{E}-06$ & $2.11 \mathrm{E}-05$ & 5.546497 & Up & choline phosphotransferase 1 \\
\hline
\end{tabular}


medRxiv preprint doi: https://doi.org/10.1101/2020.12.21.20248616; this version posted December 23, 2020. The copyright holder for this preprint (which was not certified by peer review) is the author/funder, who has granted medRxiv a license to display the preprint in perpetuity. All rights reserved. No reuse allowed without permission.

\begin{tabular}{|c|c|c|c|c|c|c|c|}
\hline ILMN_1664516 & CENPF & 2.356794 & $1.93 \mathrm{E}-06$ & $2.14 \mathrm{E}-05$ & 5.541898 & Up & centromere protein $\mathrm{F}$ \\
\hline ILMN_1721758 & ID4 & 2.117517 & $2.25 \mathrm{E}-06$ & $2.45 \mathrm{E}-05$ & 5.495085 & Up & inhibitor of DNA binding 4, HLH protein \\
\hline ILMN_2188862 & GDF15 & 2.122241 & $2.36 \mathrm{E}-06$ & $2.54 \mathrm{E}-05$ & 5.480297 & Up & growth differentiation factor 15 \\
\hline ILMN_1689400 & CLK1 & 1.887221 & $2.44 \mathrm{E}-06$ & $2.61 \mathrm{E}-05$ & 5.471046 & Up & CDC like kinase 1 \\
\hline ILMN_1691884 & STC2 & 2.388229 & $2.44 \mathrm{E}-06$ & $2.62 \mathrm{E}-05$ & 5.470024 & Up & stanniocalcin 2 \\
\hline ILMN_2149226 & CAV1 & 1.989629 & $2.56 \mathrm{E}-06$ & $2.72 \mathrm{E}-05$ & 5.455663 & Up & caveolin 1 \\
\hline ILMN_1684306 & S100A4 & 2.113301 & $3.14 \mathrm{E}-06$ & $3.23 \mathrm{E}-05$ & 5.393453 & Up & S100 calcium binding protein A4 \\
\hline ILMN_3266606 & FABP5 & 1.990411 & $3.32 \mathrm{E}-06$ & 3.39E-05 & 5.37562 & Up & fatty acid binding protein 5 \\
\hline ILMN_1809439 & HMGB1 & 1.880547 & $3.54 \mathrm{E}-06$ & $3.57 \mathrm{E}-05$ & 5.356535 & Up & high mobility group box 1 \\
\hline ILMN_1788166 & TTK & 1.9291 & $3.84 \mathrm{E}-06$ & $3.82 \mathrm{E}-05$ & 5.331466 & Up & TTK protein kinase \\
\hline ILMN_1789074 & HSPA1A & 2.074832 & $3.95 \mathrm{E}-06$ & $3.92 \mathrm{E}-05$ & 5.322514 & Up & heat shock protein family $\mathrm{A}(\mathrm{Hsp} 70)$ member $1 \mathrm{~A}$ \\
\hline ILMN_1785732 & TNFAIPG & 1.920623 & $4.34 \mathrm{E}-06$ & 4.25E-05 & 5.293922 & Up & TNF alpha induced protein 6 \\
\hline ILMN_1669032 & PPIC & 1.906199 & $4.45 \mathrm{E}-06$ & 4.34E-05 & 5.285949 & Up & peptidylprolylisomerase C \\
\hline ILMN_1715508 & NNMT & 3.181225 & $4.68 \mathrm{E}-06$ & 4.53E-05 & 5.270352 & Up & nicotinamide N-methyltransferase \\
\hline ILMN_1701308 & COL1A1 & 2.946043 & $4.92 \mathrm{E}-06$ & $4.73 \mathrm{E}-05$ & 5.255108 & Up & collagen type I alpha 1 chain \\
\hline ILMN_1801077 & PLIN2 & 2.125083 & $5.11 \mathrm{E}-06$ & $4.88 \mathrm{E}-05$ & 5.243504 & Up & perilipin 2 \\
\hline ILMN_1787026 & SEC61G & 1.980853 & $5.25 \mathrm{E}-06$ & $5 \mathrm{E}-05$ & 5.234744 & Up & SEC61 translocon gamma subunit \\
\hline ILMN_2129572 & F3 & 2.253517 & $5.28 \mathrm{E}-06$ & $5.02 \mathrm{E}-05$ & 5.23286 & Up & coagulation factor III, tissue factor \\
\hline ILMN_2130525 & TSPAN13 & 1.887651 & $5.78 \mathrm{E}-06$ & $5.44 \mathrm{E}-05$ & 5.205445 & Up & tetraspanin 13 \\
\hline ILMN_2194106 & TSPAN12 & 2.1555 & $6.39 \mathrm{E}-06$ & 5.94E-05 & 5.174066 & Up & tetraspanin 12 \\
\hline ILMN_2400759 & CPVL & 1.921761 & $6.99 \mathrm{E}-06$ & $6.41 \mathrm{E}-05$ & 5.146267 & Up & carboxypeptidasevitellogenic like \\
\hline ILMN_2349393 & MDK & 1.949982 & $7.3 \mathrm{E}-06$ & $6.63 \mathrm{E}-05$ & 5.133203 & Up & midkine \\
\hline ILMN_2412380 & TSC22D1 & 1.932728 & $7.71 \mathrm{E}-06$ & $6.95 \mathrm{E}-05$ & 5.116056 & Up & $\mathrm{TSC} 22$ domain family member 1 \\
\hline ILMN_1651958 & MGP & 2.274023 & $8.36 \mathrm{E}-06$ & $7.45 \mathrm{E}-05$ & 5.091176 & Up & matrix Gla protein \\
\hline ILMN_1782938 & SLC16A10 & 1.987142 & $1.15 \mathrm{E}-05$ & $9.76 \mathrm{E}-05$ & 4.99082 & Up & solute carrier family 16 member 10 \\
\hline ILMN_2098616 & ANXA2R & 1.895857 & $1.22 \mathrm{E}-05$ & 0.000103 & 4.972523 & Up & annexin $\mathrm{A} 2$ receptor \\
\hline ILMN_1772286 & OCIAD2 & 2.334568 & $1.32 \mathrm{E}-05$ & 0.00011 & 4.948735 & Up & OCIA domain containing 2 \\
\hline ILMN_1706505 & COL5A1 & 2.272673 & $1.48 \mathrm{E}-05$ & 0.000121 & 4.912978 & Up & collagen type $V$ alpha 1 chain \\
\hline ILMN_2189027 & LIPG & 2.033962 & $1.63 \mathrm{E}-05$ & 0.000131 & 4.883988 & Up & lipase $G$, endothelial type \\
\hline ILMN_1681737 & TMSB15A & 3.124145 & $1.63 \mathrm{E}-05$ & 0.000131 & 4.883646 & Up & thymosin beta $15 a$ \\
\hline ILMN_1656369 & TCIM & 1.932195 & $1.64 \mathrm{E}-05$ & 0.000132 & 4.881451 & Up & transcriptional and immune response regulator \\
\hline ILMN_1674386 & PITX1 & 2.237786 & $1.66 \mathrm{E}-05$ & 0.000133 & 4.87858 & Up & paired like homeodomain 1 \\
\hline ILMN_2192072 & MMP7 & 3.002907 & $1.7 \mathrm{E}-05$ & 0.000136 & 4.870958 & Up & matrix metallopeptidase 7 \\
\hline ILMN_1809590 & GINS2 & 1.914596 & $1.89 \mathrm{E}-05$ & 0.00015 & 4.83686 & Up & GINS complex subunit 2 \\
\hline ILMN_2176592 & $\mathrm{BCHE}$ & 2.002317 & $1.91 \mathrm{E}-05$ & 0.000151 & 4.834651 & Up & butyrylcholinesterase \\
\hline ILMN_1795325 & ACTG2 & 2.236952 & $2.05 \mathrm{E}-05$ & 0.00016 & 4.812345 & Up & actin gamma 2 , smooth muscle \\
\hline ILMN_2407389 & GPNMB & 2.117682 & $2.05 \mathrm{E}-05$ & 0.000161 & 4.811968 & Up & glycoprotein nmb \\
\hline ILMN_1794492 & HOXC6 & 2.052435 & $2.1 \mathrm{E}-05$ & 0.000164 & 4.80423 & Up & homeobox $\mathrm{C} 6$ \\
\hline ILMN_1811468 & IRX3 & 1.966317 & $2.3 \mathrm{E}-05$ & 0.000177 & 4.77582 & Up & iroquoishomeobox 3 \\
\hline ILMN_2131861 & SOCS2 & 1.90633 & $2.31 \mathrm{E}-05$ & 0.000177 & 4.774929 & Up & suppressor of cytokine signaling 2 \\
\hline ILMN_1706643 & COL6A3 & 2.462958 & $2.52 \mathrm{E}-05$ & 0.000191 & 4.747618 & Up & $\begin{array}{l}\text { collagen type VI alpha } 3 \text { chain } \\
\text { major histocompatibility complex, class II, DR beta }\end{array}$ \\
\hline ILMN_2066060 & HLA-DRBG & 2.154531 & $2.69 \mathrm{E}-05$ & 0.000202 & 4.726737 & Up & (pseudogene) \\
\hline ILMN_1781626 & C1s & 1.931397 & $2.82 \mathrm{E}-05$ & 0.000211 & 4.711355 & Up & complement $\mathrm{C} 1 \mathrm{~s}$ \\
\hline
\end{tabular}


medRxiv preprint doi: https://doi.org/10.1101/2020.12.21.20248616; this version posted December 23, 2020. The copyright holder for this preprint (which was not certified by peer review) is the author/funder, who has granted medRxiv a license to display the preprint in perpetuity.

All rights reserved. No reuse allowed without permission.

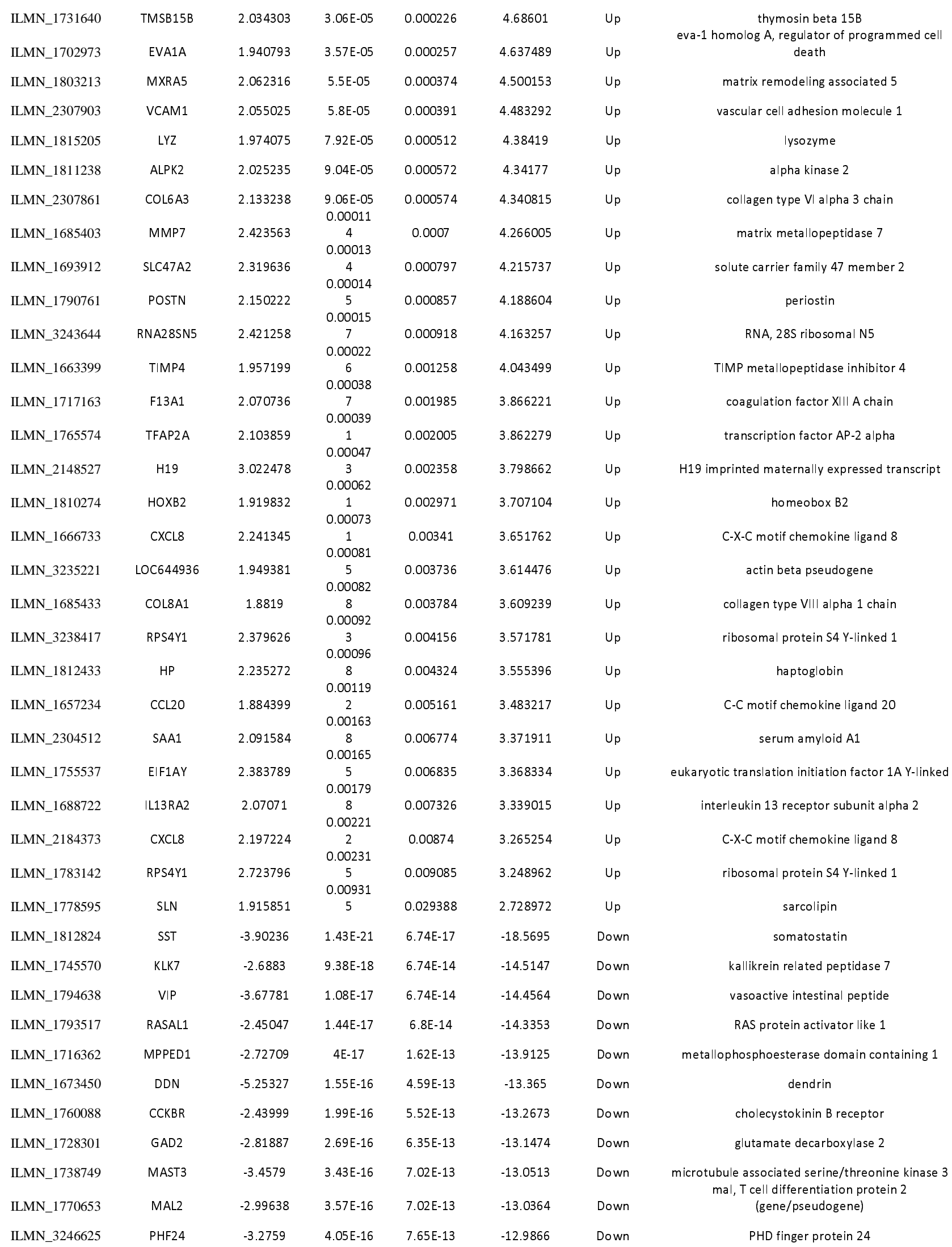


medRxiv preprint doi: https://doi.org/10.1101/2020.12.21.20248616; this version posted December 23, 2020. The copyright holder for this preprint (which was not certified by peer review) is the author/funder, who has granted medRxiv a license to display the preprint in perpetuity. All rights reserved. No reuse allowed without permission.

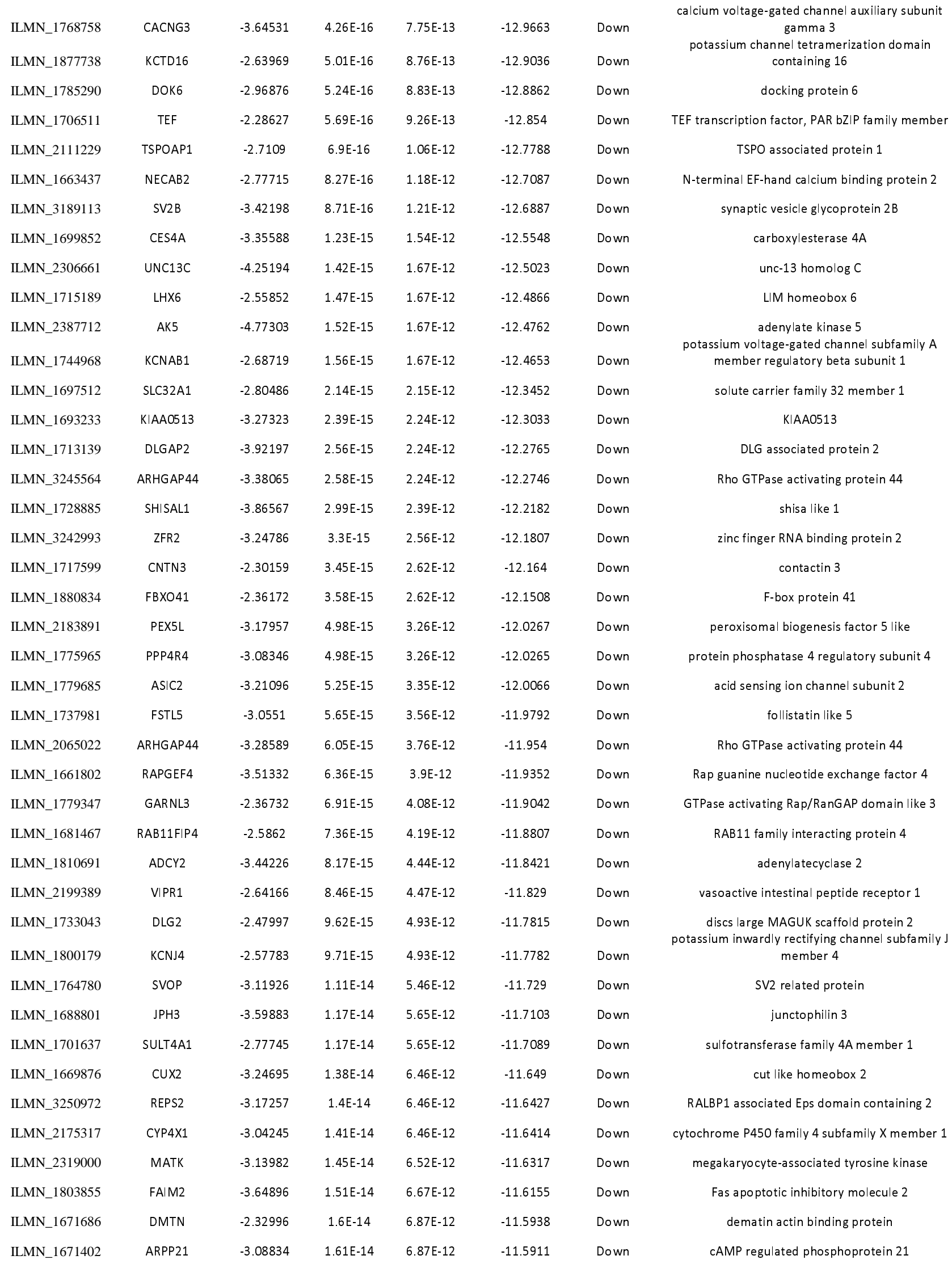


medRxiv preprint doi: https://doi.org/10.1101/2020.12.21.20248616; this version posted December 23, 2020. The copyright holder for this preprint (which was not certified by peer review) is the author/funder, who has granted medRxiv a license to display the preprint in perpetuity. All rights reserved. No reuse allowed without permission.

\begin{tabular}{|c|c|c|c|c|c|c|c|}
\hline ILMN_1755954 & CPEB3 & -2.43319 & $1.65 \mathrm{E}-14$ & $6.94 \mathrm{E}-12$ & -11.584 & Down & $\begin{array}{l}\text { cytoplasmic polyadenylation element binding } \\
\text { protein } 3\end{array}$ \\
\hline ILMN_2412822 & $\mathrm{SCN} 3 \mathrm{~B}$ & -2.90729 & $1.69 \mathrm{E}-14$ & $6.99 \mathrm{E}-12$ & -11.5736 & Down & sodium voltage-gated channel beta subunit 3 \\
\hline ILMN_1733983 & FAM153B & -2.78258 & $1.7 \mathrm{E}-14$ & $6.99 \mathrm{E}-12$ & -11.572 & Down & family with sequence similarity 153 member B \\
\hline ILMN_1723837 & SLC6A17 & -2.51648 & $1.8 \mathrm{E}-14$ & $7.2 \mathrm{E}-12$ & -11.5515 & Down & solute carrier family 6 member 17 \\
\hline ILMN_1676215 & DLG2 & -3.96266 & $1.82 \mathrm{E}-14$ & $7.21 \mathrm{E}-12$ & -11.5478 & Down & discs large MAGUK scaffold protein 2 \\
\hline ILMN_2374728 & ARPP21 & -3.11431 & $2 \mathrm{E}-14$ & $7.79 \mathrm{E}-12$ & -11.5137 & Down & cAMP regulated phosphoprotein 21 \\
\hline ILMN_1727098 & PPP1R16B & -3.85387 & $2.3 \mathrm{E}-14$ & 8.67E-12 & -11.4619 & Down & protein phosphatase 1 regulatory subunit $16 \mathrm{~B}$ \\
\hline ILMN_1743219 & CA11 & -3.32503 & $2.43 \mathrm{E}-14$ & $9.04 \mathrm{E}-12$ & -11.4416 & Down & carbonic anhydrase 11 \\
\hline ILMN_1784822 & PPP1R3F & -2.34625 & $2.6 \mathrm{E}-14$ & $9.58 \mathrm{E}-12$ & -11.4179 & Down & protein phosphatase 1 regulatory subunit $3 F$ \\
\hline ILMN_1748812 & TMEM130 & -3.01867 & $2.84 \mathrm{E}-14$ & $1.04 \mathrm{E}-11$ & -11.3857 & Down & transmembrane protein 130 \\
\hline ILMN_1698846 & SLC8A2 & -3.58539 & $3.54 \mathrm{E}-14$ & $1.25 \mathrm{E}-11$ & -11.3053 & Down & solute carrier family 8 member $\mathrm{A} 2$ \\
\hline ILMN_1712943 & CCK & -4.05777 & $3.62 \mathrm{E}-14$ & $1.27 \mathrm{E}-11$ & -11.2971 & Down & cholecystokinin \\
\hline ILMN_1878019 & DOK6 & -2.94473 & $3.7 \mathrm{E}-14$ & $1.28 \mathrm{E}-11$ & -11.2892 & Down & docking protein 6 \\
\hline ILMN_3247424 & ADAP1 & -2.31861 & 4.15E-14 & $1.39 \mathrm{E}-11$ & -11.2479 & Down & ArfGAP with dual PH domains 1 \\
\hline ILMN_1808122 & C1OTNF4 & -2.58708 & $4.51 \mathrm{E}-14$ & $1.49 \mathrm{E}-11$ & -11.2183 & Down & C1q and TNF related 4 \\
\hline ILMN_1716563 & PRKCB & -3.35285 & $5.02 \mathrm{E}-14$ & $1.65 \mathrm{E}-11$ & -11.1795 & Down & protein kinase $C$ beta \\
\hline ILMN_1796069 & CBLN2 & -2.57811 & $5.47 \mathrm{E}-14$ & $1.76 \mathrm{E}-11$ & -11.1485 & Down & cerebellin 2 precursor \\
\hline ILMN_2317580 & SHANK3 & -3.19387 & $5.65 \mathrm{E}-14$ & $1.8 \mathrm{E}-11$ & -11.137 & Down & SH3 and multiple ankyrin repeat domains 3 \\
\hline ILMN_1695978 & LINGO2 & -3.00248 & $5.77 \mathrm{E}-14$ & $1.82 \mathrm{E}-11$ & -11.1297 & Down & leucine rich repeat and Ig domain containing 2 \\
\hline ILMN_2300664 & CACNA1I & -3.03139 & $6.1 \mathrm{E}-14$ & $1.91 \mathrm{E}-11$ & -11.1098 & Down & calcium voltage-gated channel subunit alpha1 I \\
\hline ILMN_1716507 & EPB41L1 & -2.50248 & $6.27 \mathrm{E}-14$ & $1.95 \mathrm{E}-11$ & -11.1 & Down & $\begin{array}{l}\text { erythrocyte membrane protein band } 4.1 \text { like } 1 \\
\text { protein kinase } C \text { and casein kinase substrate in }\end{array}$ \\
\hline ILMN_1751020 & PACSIN1 & -5.28035 & $7.4 \mathrm{E}-14$ & 2.27E-11 & -11.0405 & Down & neurons 1 \\
\hline ILMN_1697561 & FBXL16 & -4.08049 & $8.93 \mathrm{E}-14$ & 2.64E-11 & -10.9736 & Down & F-box and leucine rich repeat protein 16 \\
\hline ILMN_1658094 & ZNF365 & -3.01422 & $1.04 \mathrm{E}-13$ & $2.97 \mathrm{E}-11$ & -10.9199 & Down & zinc finger protein 365 \\
\hline ILMN_1698148 & CHD5 & -4.65587 & $1.09 \mathrm{E}-13$ & 3.07E-11 & -10.9029 & Down & chromodomain helicase DNA binding protein 5 \\
\hline ILMN_1777650 & TMEM151B & -2.2704 & $1.09 \mathrm{E}-13$ & 3.07E-11 & -10.9017 & Down & transmembrane protein $151 \mathrm{~B}$ \\
\hline ILMN_1730546 & GNAO1 & -3.49137 & $1.23 \mathrm{E}-13$ & $3.39 \mathrm{E}-11$ & -10.8605 & Down & G protein subunit alpha 01 \\
\hline ILMN_1757081 & SYN2 & -4.03639 & $1.34 \mathrm{E}-13$ & 3.63E-11 & -10.8294 & Down & synapsin II \\
\hline ILMN_1668125 & MYRIP & -2.43612 & $1.51 \mathrm{E}-13$ & 3.89E-11 & -10.7866 & Down & $\begin{array}{l}\text { myosin VIIA and Rab interacting protein } \\
\text { potassium calcium-activated channel subfamily M }\end{array}$ \\
\hline ILMN_1740508 & KCNMA1 & -2.92777 & $1.53 \mathrm{E}-13$ & 3.89E-11 & -10.7839 & Down & alpha 1 \\
\hline ILMN_1653687 & GALNT9 & -3.00871 & $1.54 \mathrm{E}-13$ & $3.91 \mathrm{E}-11$ & -10.7806 & Down & polypeptide $\mathrm{N}$-acetylgalactosaminyltransferase 9 \\
\hline ILMN_1654632 & RGS7BP & -2.54813 & $1.61 \mathrm{E}-13$ & 4.03E-11 & -10.7642 & Down & $\begin{array}{l}\text { regulator of } \mathrm{G} \text { protein signaling } 7 \text { binding protein } \\
\text { calcium/calmodulin dependent protein kinase II }\end{array}$ \\
\hline ILMN_1666445 & CAMK2A & -5.25014 & $1.76 \mathrm{E}-13$ & 4.26E-11 & -10.734 & Down & alpha \\
\hline ILMN_1809947 & TMEM155 & -2.63279 & $1.78 \mathrm{E}-13$ & 4.29E-11 & -10.7293 & Down & transmembrane protein 155 \\
\hline ILMN_2218758 & NECAB1 & -3.5477 & $1.8 \mathrm{E}-13$ & $4.31 \mathrm{E}-11$ & -10.726 & Down & $\mathrm{N}$-terminal EF-hand calcium binding protein 1 \\
\hline ILMN_1819608 & ARRB1 & -3.0374 & $1.81 \mathrm{E}-13$ & 4.32E-11 & -10.723 & Down & $\begin{array}{c}\text { arrestin beta } 1 \\
\text { glucose-fructose oxidoreductase domain containing }\end{array}$ \\
\hline ILMN_1778240 & GFOD1 & -2.59766 & $1.84 \mathrm{E}-13$ & 4.33E-11 & -10.7186 & Down & 1 \\
\hline ILMN_1679532 & GRM3 & -3.84381 & $1.88 \mathrm{E}-13$ & 4.42E-11 & -10.7098 & Down & $\begin{array}{l}\text { glutamate metabotropic receptor } 3 \\
\text { sosondowahankyrin repeat domain family mem ber }\end{array}$ \\
\hline ILMN_1779882 & SOWAHA & -3.53594 & $1.9 \mathrm{E}-13$ & 4.44E-11 & -10.7067 & Down & $\begin{array}{c}\mathrm{A} \\
\text { ArfGAP with GTPase domain, ankyrin repeat and } \mathrm{PH}\end{array}$ \\
\hline ILMN_2269136 & AGAP3 & -2.56361 & $2 \mathrm{E}-13$ & $4.6 \mathrm{E}-11$ & -10.6893 & Down & domain 3 \\
\hline ILMN_1805404 & GRIN1 & -4.72076 & $2.02 \mathrm{E}-13$ & 4.62E-11 & -10.6856 & Down & glutamate ionotropic receptor NMDA type subunit 1 \\
\hline ILMN_1665257 & RIMS2 & -2.83656 & $2.18 \mathrm{E}-13$ & 4.87E-11 & -10.6585 & Down & regulating synaptic membrane exocytosis 2 \\
\hline
\end{tabular}


medRxiv preprint doi: https://doi.org/10.1101/2020.12.21.20248616; this version posted December 23, 2020. The copyright holder for this preprint (which was not certified by peer review) is the author/funder, who has granted medRxiv a license to display the preprint in perpetuity. All rights reserved. No reuse allowed without permission.

\begin{tabular}{|c|c|c|c|c|c|c|c|}
\hline ILMN_1676283 & RIMBP2 & -2.69868 & $2.28 \mathrm{E}-13$ & $5 \mathrm{E}-11$ & -10.6431 & Down & RIMS binding protein 2 \\
\hline ILMN_1767129 & $\mathrm{ABCC} 8$ & -2.83221 & $2.38 \mathrm{E}-13$ & $5.21 \mathrm{E}-11$ & -10.6267 & Down & ATP binding cassette subfamily $C$ member 8 \\
\hline ILMN_1856533 & CDKL2 & -2.25591 & $2.71 \mathrm{E}-13$ & $5.74 \mathrm{E}-11$ & -10.5819 & Down & cyclin dependent kinase like 2 \\
\hline ILMN_2049672 & ANO3 & -2.58408 & $2.84 \mathrm{E}-13$ & $5.95 \mathrm{E}-11$ & -10.5657 & Down & $\operatorname{anoctamin} 3$ \\
\hline ILMN_1758825 & ABLIM2 & -2.9389 & $2.93 \mathrm{E}-13$ & $6.06 \mathrm{E}-11$ & -10.5552 & Down & $\begin{array}{l}\text { actin binding LIM protein family member } 2 \\
\text { potassium channel tetramerization domain }\end{array}$ \\
\hline ILMN_1798557 & KCTD16 & -3.8098 & $2.97 \mathrm{E}-13$ & $6.09 \mathrm{E}-11$ & -10.5504 & Down & containing 16 \\
\hline ILMN_1811835 & SLC12A5 & -2.55396 & $2.98 \mathrm{E}-13$ & $6.1 \mathrm{E}-11$ & -10.5483 & Down & $\begin{array}{l}\text { solute carrier family } 12 \text { member } 5 \\
\text { potassium voltage-gated channel subfamily H }\end{array}$ \\
\hline ILMN_1704063 & $\mathrm{KCNH3}$ & -2.77591 & $3.09 \mathrm{E}-13$ & $6.29 \mathrm{E}-11$ & -10.5356 & Down & member 3 \\
\hline ILMN_1801090 & KRT222 & -2.3865 & $3.25 \mathrm{E}-13$ & $6.51 \mathrm{E}-11$ & -10.5182 & Down & keratin 222 \\
\hline ILMN_1669982 & CCDC85A & -2.86293 & $3.74 \mathrm{E}-13$ & 7.27E-11 & -10.4696 & Down & coiled-coil domain containing $85 \mathrm{~A}$ \\
\hline ILMN_1802646 & EPHB6 & -2.56826 & $4.08 \mathrm{E}-13$ & $7.83 \mathrm{E}-11$ & -10.4395 & Down & EPH receptor $\mathrm{B} 6$ \\
\hline ILMN_3299520 & PRKCB & -3.64924 & $4.22 \mathrm{E}-13$ & 8.01E-11 & -10.4273 & Down & protein kinase $C$ beta \\
\hline ILMN_2317581 & SHANK3 & -3.145 & 4.5E-13 & 8.47E-11 & -10.4052 & Down & SH3 and multiple ankyrin repeat domains 3 \\
\hline ILMN_1798587 & ARHGEF7 & -2.78968 & $4.52 \mathrm{E}-13$ & 8.47E-11 & -10.404 & Down & Rho guanine nucleotide exchange factor 7 \\
\hline ILMN_1727805 & SYNGR1 & -2.63613 & $4.62 \mathrm{E}-13$ & $8.62 \mathrm{E}-11$ & -10.3966 & Down & synaptogyrin 1 \\
\hline ILMN_1681356 & PDE2A & -2.61305 & $4.88 \mathrm{E}-13$ & $8.98 \mathrm{E}-11$ & -10.377 & Down & phosphodiesterase $2 \mathrm{~A}$ \\
\hline ILMN_1814790 & SHANK2 & -2.63076 & $5.18 \mathrm{E}-13$ & $9.38 \mathrm{E}-11$ & -10.3565 & Down & SH3 and multiple ankyrin repeat domains 2 \\
\hline ILMN_2181125 & NAPB & -3.19802 & $5.29 \mathrm{E}-13$ & $9.53 \mathrm{E}-11$ & -10.3491 & Down & $\begin{array}{l}\text { NSF attachment protein beta } \\
\text { oligodendrocytic myelin paranodal and inner loop }\end{array}$ \\
\hline ILMN_1666090 & OPALIN & -4.96328 & $5.31 \mathrm{E}-13$ & $9.53 \mathrm{E}-11$ & -10.3482 & Down & protein \\
\hline ILMN_1697189 & PNCK & -3.46672 & $5.34 \mathrm{E}-13$ & $9.55 \mathrm{E}-11$ & -10.3461 & Down & pregnancy up-regulated nonubiquitousCaM kinase \\
\hline ILMN_3234081 & STX1B & -3.64187 & $5.99 \mathrm{E}-13$ & $1.03 \mathrm{E}-10$ & -10.3065 & Down & $\begin{array}{l}\text { syntaxin 1B } \\
\text { oligodendrocytic myelin paranodal and inner loop }\end{array}$ \\
\hline ILMN_2373236 & OPALIN & -4.93327 & $6.05 \mathrm{E}-13$ & $1.04 \mathrm{E}-10$ & -10.3029 & Down & protein \\
\hline ILMN_1821473 & OTUD7A & -2.77145 & $7.52 \mathrm{E}-13$ & $1.26 \mathrm{E}-10$ & -10.2278 & Down & OTU deubiquitinase $7 \mathrm{~A}$ \\
\hline ILMN_1781060 & SYN2 & -3.09381 & $8.03 \mathrm{E}-13$ & $1.31 \mathrm{E}-10$ & -10.2053 & Down & synapsin II \\
\hline ILMN_1697267 & PRKCZ & -2.75451 & $8.04 \mathrm{E}-13$ & $1.31 \mathrm{E}-10$ & -10.2051 & Down & protein kinase $\mathrm{C}$ zeta \\
\hline ILMN_2080080 & MAP7D2 & -2.65203 & $8.75 \mathrm{E}-13$ & $1.42 \mathrm{E}-10$ & -10.176 & Down & MAP7 domain containing 2 \\
\hline ILMN_1801703 & CPLX1 & -3.44343 & $8.8 \mathrm{E}-13$ & $1.42 \mathrm{E}-10$ & -10.1739 & Down & complexin 1 \\
\hline ILMN_1722037 & PEX5L & -2.48335 & $8.83 \mathrm{E}-13$ & $1.42 \mathrm{E}-10$ & -10.1726 & Down & peroxisomal biogenesis factor 5 like \\
\hline ILMN_1753490 & JAKMIP3 & -3.06663 & $9.34 \mathrm{E}-13$ & $1.47 \mathrm{E}-10$ & -10.1537 & Down & Janus kinase and microtubule interacting protein 3 \\
\hline ILMN_1806908 & PRKCB & -3.24529 & $9.82 \mathrm{E}-13$ & $1.52 \mathrm{E}-10$ & -10.1364 & Down & protein kinase $\mathrm{C}$ beta \\
\hline ILMN_1760246 & BSN & -3.60529 & $9.93 \mathrm{E}-13$ & $1.53 \mathrm{E}-10$ & -10.1326 & Down & bassoon presynaptic cytomatrix protein \\
\hline ILMN_1770629 & SLC17A7 & -4.45809 & $1.04 \mathrm{E}-12$ & $1.59 \mathrm{E}-10$ & -10.1161 & Down & solute carrier family 17 member 7 \\
\hline ILMN_1736976 & HPCAL4 & -3.95599 & $1.05 \mathrm{E}-12$ & $1.59 \mathrm{E}-10$ & -10.1124 & Down & hippocalcin like 4 \\
\hline ILMN_1702789 & KNDC1 & -3.53329 & $1.09 \mathrm{E}-12$ & $1.64 \mathrm{E}-10$ & -10.0994 & Down & kinase non-catalytic $\mathrm{C}$-lobe domain containing 1 \\
\hline ILMN_1657497 & KIAA0319 & -2.65965 & $1.09 \mathrm{E}-12$ & $1.64 \mathrm{E}-10$ & -10.0991 & Down & KIAA0319 \\
\hline ILMN_1771622 & CALY & -3.97864 & $1.12 \mathrm{E}-12$ & $1.66 \mathrm{E}-10$ & -10.0926 & Down & calcyon neuron specific vesicular protein \\
\hline ILMN_1676998 & SCN2B & -2.91677 & $1.45 \mathrm{E}-12$ & $2.11 \mathrm{E}-10$ & -10.0034 & Down & sodium voltage-gated channel beta subunit 2 \\
\hline ILMN_1685582 & MYT1L & -3.57575 & $1.53 \mathrm{E}-12$ & $2.2 \mathrm{E}-10$ & -9.98515 & Down & myelin transcription factor 1 like \\
\hline ILMN_2047511 & ADAP1 & -3.2785 & $1.62 \mathrm{E}-12$ & $2.3 \mathrm{E}-10$ & -9.96587 & Down & ArfGAP with dual PH domains 1 \\
\hline ILMN_1705686 & NRGN & -3.67841 & $1.72 \mathrm{E}-12$ & $2.4 \mathrm{E}-10$ & -9.94419 & Down & neurogranin \\
\hline ILMN_1783805 & PNMA3 & -3.0511 & $1.77 \mathrm{E}-12$ & $2.45 \mathrm{E}-10$ & -9.93513 & Down & PNMA family member 3 \\
\hline ILMN_2096623 & SLITRK1 & -3.11407 & $2.28 \mathrm{E}-12$ & $3.04 \mathrm{E}-10$ & -9.84945 & Down & SLIT and NTRK like family member 1 \\
\hline
\end{tabular}


medRxiv preprint doi: https://doi.org/10.1101/2020.12.21.20248616; this version posted December 23, 2020. The copyright holder for this preprint (which was not certified by peer review) is the author/funder, who has granted medRxiv a license to display the preprint in perpetuity. All rights reserved. No reuse allowed without permission.

\begin{tabular}{|c|c|c|c|c|c|c|c|}
\hline ILMN_2215989 & NEFM & -3.23687 & $2.32 \mathrm{E}-12$ & $3.08 \mathrm{E}-10$ & -9.84349 & Down & neurofilament medium \\
\hline ILMN_2129910 & SLC12A5 & -4.78899 & $2.34 \mathrm{E}-12$ & $3.09 \mathrm{E}-10$ & -9.8411 & Down & solute carrier family 12 member 5 \\
\hline ILMN_2414878 & STXBP1 & -2.50892 & $2.5 \mathrm{E}-12$ & $3.24 \mathrm{E}-10$ & -9.81853 & Down & syntaxin binding protein 1 \\
\hline ILMN_3234654 & PCLO & -3.08972 & $2.54 \mathrm{E}-12$ & $3.27 \mathrm{E}-10$ & -9.81314 & Down & piccolo presynaptic cytomatrix protein \\
\hline ILMN_2411864 & PHACTR3 & -2.69365 & $2.64 \mathrm{E}-12$ & 3.36E-10 & -9.80006 & Down & phosphatase and actin regulator 3 \\
\hline ILMN_1803256 & STOX2 & -2.40057 & $2.65 \mathrm{E}-12$ & $3.36 \mathrm{E}-10$ & -9.79863 & Down & storkhead box 2 \\
\hline ILMN_1718671 & GJB6 & -3.53106 & $2.7 \mathrm{E}-12$ & $3.41 \mathrm{E}-10$ & -9.7922 & Down & gap junction protein beta 6 \\
\hline ILMN_1759563 & NRIP3 & -2.68456 & $2.73 \mathrm{E}-12$ & $3.43 \mathrm{E}-10$ & -9.78917 & Down & $\begin{array}{l}\text { nuclear receptor interacting protein } 3 \\
\text { potassium voltage-gated channel subfamily Q }\end{array}$ \\
\hline ILMN_1788663 & KCNQ2 & -4.09375 & $2.82 \mathrm{E}-12$ & $3.5 \mathrm{E}-10$ & -9.77768 & Down & member 2 \\
\hline ILMN_1756784 & NCS1 & -3.20633 & $3.14 \mathrm{E}-12$ & $3.79 \mathrm{E}-10$ & -9.74145 & Down & neuronal calcium sensor 1 \\
\hline ILMN_1714577 & OGDHL & -2.36145 & $3.23 \mathrm{E}-12$ & $3.9 \mathrm{E}-10$ & -9.73145 & Down & oxoglutarate dehydrogenase like \\
\hline ILMN_1657361 & $\mathrm{CBX7}$ & -2.41733 & $3.25 \mathrm{E}-12$ & $3.9 \mathrm{E}-10$ & -9.73018 & Down & chromobox 7 \\
\hline ILMN_1754126 & SH2D5 & -2.27744 & $3.31 \mathrm{E}-12$ & $3.96 \mathrm{E}-10$ & -9.72324 & Down & $\begin{array}{l}\text { SH2 domain containing } 5 \\
\text { calcium/calmodulin dependent protein kinase }\end{array}$ \\
\hline ILMN_1743021 & CAMKK2 & -2.25343 & $3.35 \mathrm{E}-12$ & $4 \mathrm{E}-10$ & -9.71946 & Down & kinase 2 \\
\hline ILMN_1860963 & RNF130 & -2.86701 & $3.47 \mathrm{E}-12$ & $4.08 \mathrm{E}-10$ & -9.70802 & Down & ring finger protein 130 \\
\hline ILMN_1769195 & ERC2 & -2.39088 & $3.62 \mathrm{E}-12$ & $4.21 \mathrm{E}-10$ & -9.6933 & Down & ELKS/RAB6-interacting/CAST family member 2 \\
\hline ILMN_1695941 & NECAB1 & -2.53708 & $3.95 \mathrm{E}-12$ & $4.52 \mathrm{E}-10$ & -9.66415 & Down & $\mathrm{N}$-terminal EF-hand calcium binding protein 1 \\
\hline ILMN_1776998 & DNAJA4 & -2.31341 & $4.02 \mathrm{E}-12$ & $4.58 \mathrm{E}-10$ & -9.65833 & Down & DnaJ heat shock protein family (Hsp40) member A4 \\
\hline ILMN_2172969 & STXBP6 & -3.05855 & $4.16 \mathrm{E}-12$ & 4.69E-10 & -9.64668 & Down & syntaxin binding protein 6 \\
\hline ILMN_3253857 & SCN8A & -2.49979 & $4.21 \mathrm{E}-12$ & 4.74E-10 & -9.64265 & Down & sodium voltage-gated channel alpha subunit 8 \\
\hline ILMN_1712352 & DOCK3 & -2.3189 & $4.45 \mathrm{E}-12$ & $4.91 \mathrm{E}-10$ & -9.62437 & Down & dedicator of cytokinesis 3 \\
\hline ILMN_1701558 & MAP1A & -2.88131 & 4.49E-12 & $4.92 \mathrm{E}-10$ & -9.62123 & Down & microtubule associated protein $1 \mathrm{~A}$ \\
\hline ILMN_1683998 & CREG2 & -2.9733 & $4.63 \mathrm{E}-12$ & $5.02 \mathrm{E}-10$ & -9.61098 & Down & cellular repressor of E1A stimulated genes 2 \\
\hline ILMN_1762207 & SGSM1 & -2.85143 & 4.79E-12 & $5.11 \mathrm{E}-10$ & -9.59924 & Down & $\begin{array}{l}\text { small G protein signaling modulator } 1 \\
\text { protein phosphatase } 1 \text { regulatory inhibitor subunit }\end{array}$ \\
\hline ILMN_2056606 & PPP1R1A & -2.44471 & 4.93E-12 & $5.22 \mathrm{E}-10$ & -9.59005 & Down & $1 \mathrm{~A}$ \\
\hline ILMN_1763612 & TMEM132D & -3.02906 & $5.61 \mathrm{E}-12$ & $5.86 \mathrm{E}-10$ & -9.54699 & Down & transmembrane protein $132 \mathrm{D}$ \\
\hline ILMN_1697433 & CYP46A1 & -2.88311 & $6.49 \mathrm{E}-12$ & $6.61 \mathrm{E}-10$ & -9.49807 & Down & cytochrome P450 family 46 subfamily A member 1 \\
\hline ILMN_1717029 & RTP5 & -3.46102 & $6.6 \mathrm{E}-12$ & $6.67 \mathrm{E}-10$ & -9.49259 & Down & receptor transporter protein 5 (putative) \\
\hline ILMN_1806306 & SV2B & -3.46821 & $6.7 \mathrm{E}-12$ & $6.77 \mathrm{E}-10$ & -9.4873 & Down & synaptic vesicle glycoprotein $2 B$ \\
\hline ILMN_1715927 & VSTM2L & -2.27581 & $7.46 \mathrm{E}-12$ & $7.44 \mathrm{E}-10$ & -9.45152 & Down & V-set and transmembrane domain containing 2 like \\
\hline ILMN_1793435 & KIAA1107 & -2.56635 & $7.5 \mathrm{E}-12$ & $7.46 \mathrm{E}-10$ & -9.44996 & Down & KIAA1107 \\
\hline ILMN_1738058 & CPNE6 & -3.71607 & $7.72 \mathrm{E}-12$ & $7.63 \mathrm{E}-10$ & -9.44021 & Down & copine 6 \\
\hline ILMN_2400947 & CELF2 & -2.31667 & $7.83 \mathrm{E}-12$ & $7.71 \mathrm{E}-10$ & -9.4355 & Down & CUGBP Elav-like family member 2 \\
\hline ILMN_1679984 & $\mathrm{ZCCHC12}$ & -2.67824 & $8.27 \mathrm{E}-12$ & $8.11 \mathrm{E}-10$ & -9.41726 & Down & zinc finger $\mathrm{CCHC}$-type containing 12 \\
\hline ILMN_1674817 & C1orf115 & -3.1965 & $8.55 \mathrm{E}-12$ & $8.32 \mathrm{E}-10$ & -9.40649 & Down & chromosome 1 open reading frame 115 \\
\hline ILMN_1758829 & $\mathrm{JPH} 4$ & -2.66411 & $9.53 \mathrm{E}-12$ & $9.08 \mathrm{E}-10$ & -9.37029 & Down & junctophilin 4 \\
\hline ILMN_1737162 & LGI3 & -2.40372 & $9.8 \mathrm{E}-12$ & $9.31 \mathrm{E}-10$ & -9.36106 & Down & leucine rich repeat LGI family member 3 \\
\hline ILMN_1698885 & PTPRT & -2.91444 & $9.88 \mathrm{E}-12$ & $9.37 \mathrm{E}-10$ & -9.35837 & Down & $\begin{array}{l}\text { protein tyrosine phosphatase receptor type } \mathrm{T} \\
\text { ArfGAP with GTPase domain, ankyrin repeat and } \mathrm{PH}\end{array}$ \\
\hline ILMN_1857897 & AGAP1 & -2.46817 & $1.07 \mathrm{E}-11$ & $1 \mathrm{E}-09$ & -9.33151 & Down & domain 1 \\
\hline ILMN_1756715 & RUNDC3A & -3.92779 & $1.11 \mathrm{E}-11$ & $1.03 \mathrm{E}-09$ & -9.32117 & Down & $\begin{array}{l}\text { RUN domain containing } 3 \mathrm{~A} \\
\text { ankyrin repeat and sterile alpha motif domain }\end{array}$ \\
\hline ILMN_1758392 & ANKS1B & -3.80973 & $1.18 \mathrm{E}-11$ & $1.08 \mathrm{E}-09$ & -9.29975 & Down & containing $1 \mathrm{~B}$ \\
\hline ILMN_2078975 & GRM3 & -4.19654 & 1.19E-11 & $1.09 \mathrm{E}-09$ & -9.29692 & Down & glutamate metabotropic receptor 3 \\
\hline
\end{tabular}


medRxiv preprint doi: https://doi.org/10.1101/2020.12.21.20248616; this version posted December 23, 2020. The copyright holder for this preprint (which was not certified by peer review) is the author/funder, who has granted medRxiv a license to display the preprint in perpetuity. All rights reserved. No reuse allowed without permission.

\begin{tabular}{|c|c|c|c|c|c|c|c|}
\hline ILMN_1734695 & MAP4 & -3.57608 & $1.19 \mathrm{E}-11$ & 1.09E-09 & -9.29666 & Down & $\begin{array}{c}\text { microtubule associated protein } 4 \\
\text { gamma-aminobutyric acid type A receptor gamma2 }\end{array}$ \\
\hline ILMN_2413964 & GABRG2 & -2.26465 & $1.29 \mathrm{E}-11$ & $1.16 \mathrm{E}-09$ & -9.27099 & Down & subunit \\
\hline ILMN_1748434 & LOC283683 & -2.86812 & $1.33 \mathrm{E}-11$ & 1.19E-09 & -9.25976 & Down & $\begin{array}{c}\text { uncharacterized LOC283683 } \\
\text { protein phosphatase } 1 \text { regulatory inhibitor subunit }\end{array}$ \\
\hline ILMN_2304495 & PPP1R1B & -2.99976 & $1.44 \mathrm{E}-11$ & $1.26 \mathrm{E}-09$ & -9.2349 & Down & 1B \\
\hline ILMN_1715496 & HTR2A & -3.33624 & $1.87 \mathrm{E}-11$ & $1.56 \mathrm{E}-09$ & -9.14744 & Down & 5-hydroxytryptamine receptor $2 \mathrm{~A}$ \\
\hline ILMN_1803018 & $\mathrm{KIFC} 2$ & -2.45136 & $2.02 \mathrm{E}-11$ & $1.67 \mathrm{E}-09$ & -9.12265 & Down & kinesin family member $\mathrm{C} 2$ \\
\hline ILMN_2188722 & GLS & -2.54535 & $2.02 \mathrm{E}-11$ & $1.67 \mathrm{E}-09$ & -9.12162 & Down & glutaminase \\
\hline ILMN_1760990 & SH3GL3 & -4.43941 & $2.08 \mathrm{E}-11$ & 1.7E-09 & -9.11275 & Down & $\begin{array}{l}\text { SH3 domain containing GRB2 like } 3 \text {, endophilin A3 } \\
\text { gamma-aminobutyric acid type A receptor alpha5 }\end{array}$ \\
\hline ILMN_1707571 & GABRA5 & -2.9238 & $2.09 \mathrm{E}-11$ & 1.7E-09 & -9.11076 & Down & $\begin{array}{l}\text { subunit } \\
\text { extracellular leucine rich repeat and fibronectin }\end{array}$ \\
\hline ILMN_1658685 & ELFN2 & -2.88883 & $2.61 \mathrm{E}-11$ & $2.04 \mathrm{E}-09$ & -9.03809 & Down & type III domain containing 2 \\
\hline ILMN_2407703 & SYN1 & -4.11487 & $2.83 \mathrm{E}-11$ & $2.17 \mathrm{E}-09$ & -9.01183 & Down & synapsin I \\
\hline ILMN_1660501 & $\mathrm{LY} 6 \mathrm{H}$ & -3.56249 & $3.08 \mathrm{E}-11$ & $2.34 \mathrm{E}-09$ & -8.9841 & Down & Iymphocyte antigen 6 family member $\mathrm{H}$ \\
\hline ILMN_2237252 & $\mathrm{LY} 6 \mathrm{H}$ & -3.58074 & $3.08 \mathrm{E}-11$ & $2.34 \mathrm{E}-09$ & -8.98389 & Down & Iymphocyte antigen 6 family member $\mathrm{H}$ \\
\hline ILMN_1768595 & DLG4 & -2.61212 & $3.36 \mathrm{E}-11$ & $2.5 \mathrm{E}-09$ & -8.95567 & Down & discs large MAGUK scaffold protein 4 \\
\hline ILMN_1787658 & MTMR7 & -2.38955 & $3.52 \mathrm{E}-11$ & $2.6 \mathrm{E}-09$ & -8.94084 & Down & myotubularin related protein 7 \\
\hline ILMN_1770641 & KLHL3 & -2.52514 & $3.65 \mathrm{E}-11$ & 2.69E-09 & -8.92884 & Down & kelch like family member 3 \\
\hline ILMN_1690223 & CNTNAP2 & -4.02987 & 4.07E-11 & $2.93 \mathrm{E}-09$ & -8.89377 & Down & contactin associated protein like 2 \\
\hline ILMN_1760160 & STX1A & -2.71212 & $4.08 \mathrm{E}-11$ & $2.93 \mathrm{E}-09$ & -8.89258 & Down & syntaxin $1 \mathrm{~A}$ \\
\hline ILMN_1815033 & HPCA & -2.58013 & $4.09 \mathrm{E}-11$ & $2.93 \mathrm{E}-09$ & -8.89216 & Down & hippocalcin \\
\hline ILMN_1702255 & KCNT1 & -3.89864 & $4.55 \mathrm{E}-11$ & $3.21 \mathrm{E}-09$ & -8.85743 & Down & member 1 \\
\hline ILMN_1751569 & ERICH3 & -2.33418 & 4.7E-11 & 3.3E-09 & -8.84684 & Down & glutamate rich 3 \\
\hline ILMN_1840175 & MIR124-2HG & -3.43138 & $5.23 \mathrm{E}-11$ & $3.59 \mathrm{E}-09$ & -8.81181 & Down & $\begin{array}{l}\text { MIR124-2 host gene } \\
\text { potassium voltage-gated channel interacting protein }\end{array}$ \\
\hline ILMN_1744403 & KCNIP3 & -2.42889 & $5.37 \mathrm{E}-11$ & $3.67 \mathrm{E}-09$ & -8.80335 & Down & 3 \\
\hline ILMN_1805200 & DNM1 & -2.3268 & $5.75 \mathrm{E}-11$ & $3.88 \mathrm{E}-09$ & -8.78124 & Down & $\begin{array}{c}\text { dynamin } 1 \\
\text { gamma-aminobutyric acid type A receptor alpha5 }\end{array}$ \\
\hline ILMN_2215730 & GABRA5 & -2.36644 & $6.4 \mathrm{E}-11$ & $4.22 \mathrm{E}-09$ & -8.74627 & Down & subunit \\
\hline ILMN_1742881 & SYT1 & -3.87571 & $6.51 \mathrm{E}-11$ & 4.29E-09 & -8.74075 & Down & $\begin{array}{c}\text { synaptotagmin } 1 \\
\text { potassium two pore domain channel subfamily } \mathrm{K}\end{array}$ \\
\hline ILMN_1721027 & KCNK4 & -2.67356 & 7.27E-11 & 4.7E-09 & -8.70539 & Down & member 4 \\
\hline ILMN_1818677 & LINC00599 & -2.76459 & $7.42 \mathrm{E}-11$ & $4.78 \mathrm{E}-09$ & -8.69887 & Down & long intergenic non-protein coding RNA 599 \\
\hline ILMN_1803318 & PPFIA2 & -2.65619 & $7.59 \mathrm{E}-11$ & $4.88 \mathrm{E}-09$ & -8.69149 & Down & PTPRF interacting protein alpha 2 \\
\hline ILMN_1757807 & ETNPPL & -3.93108 & $7.61 \mathrm{E}-11$ & $4.89 \mathrm{E}-09$ & -8.69041 & Down & ethanolamine-phosphate phospho-lyase \\
\hline ILMN_2381938 & ATP2B2 & -2.27147 & $8.85 E-11$ & $5.57 \mathrm{E}-09$ & -8.6418 & Down & ATPase plasma membrane $\mathrm{Ca} 2+$ transporting 2 \\
\hline ILMN_2230566 & RAB40B & -2.37969 & $9.22 \mathrm{E}-11$ & $5.77 \mathrm{E}-09$ & -8.6286 & Down & RAB40B, member RAS oncogene family \\
\hline ILMN_1687268 & SEC14L5 & -4.19485 & $9.34 \mathrm{E}-11$ & $5.82 \mathrm{E}-09$ & -8.6243 & Down & SEC14 like lipid binding 5 \\
\hline ILMN_1713846 & PPM1H & -2.43385 & $1.08 \mathrm{E}-10$ & $6.53 \mathrm{E}-09$ & -8.57698 & Down & protein phosphatase, $\mathrm{Mg} 2+/ \mathrm{Mn} 2+$ dependent $1 \mathrm{H}$ \\
\hline ILMN_2185845 & BRSK1 & -2.42633 & $1.1 \mathrm{E}-10$ & $6.59 \mathrm{E}-09$ & -8.57072 & Down & BR serine/threonine kinase 1 \\
\hline ILMN_2174341 & GPR83 & -3.02396 & $1.11 \mathrm{E}-10$ & 6.59E-09 & -8.56947 & Down & G protein-coupled receptor 83 \\
\hline ILMN_1708223 & PAK6 & -2.44812 & $1.16 \mathrm{E}-10$ & $6.83 \mathrm{E}-09$ & -8.55433 & Down & p21 (RAC1) activated kinase 6 \\
\hline ILMN_2386982 & PRKCZ & -3.05564 & $1.17 \mathrm{E}-10$ & $6.88 \mathrm{E}-09$ & -8.55091 & Down & protein kinase $C$ zeta \\
\hline
\end{tabular}


medRxiv preprint doi: https://doi.org/10.1101/2020.12.21.20248616; this version posted December 23, 2020. The copyright holder for this preprint (which was not certified by peer review) is the author/funder, who has granted medRxiv a license to display the preprint in perpetuity. All rights reserved. No reuse allowed without permission.

\begin{tabular}{|c|c|c|c|c|c|c|c|}
\hline ILMN_1680507 & PPP2R2C & -3.64903 & $1.2 \mathrm{E}-10$ & $7.03 \mathrm{E}-09$ & -8.54264 & Down & protein phosphatase 2 regulatory subunit Bgamma \\
\hline ILMN_1732782 & SCN2A & -3.20253 & $1.22 \mathrm{E}-10$ & 7.13E-09 & -8.53731 & Down & sodium voltage-gated channel alpha subunit 2 \\
\hline ILMN_1676413 & VSNL1 & -4.32357 & $1.39 \mathrm{E}-10$ & $7.92 \mathrm{E}-09$ & -8.49761 & Down & visinin like 1 \\
\hline ILMN_2277537 & MUTYH & -2.38479 & $1.42 \mathrm{E}-10$ & $8.07 \mathrm{E}-09$ & -8.48911 & Down & mutY DNA glycosylase \\
\hline ILMN_1886092 & FAM189A1 & -2.40906 & $1.42 \mathrm{E}-10$ & $8.07 \mathrm{E}-09$ & -8.48879 & Down & family with sequence similarity 189 member A1 \\
\hline ILMN_3297876 & VSTM2B & -3.06646 & $1.44 \mathrm{E}-10$ & $8.12 \mathrm{E}-09$ & -8.48578 & Down & V-set and transmembrane domain containing 2B \\
\hline ILMN_1805807 & SLC30A3 & -3.27641 & $1.51 \mathrm{E}-10$ & $8.45 \mathrm{E}-09$ & -8.46968 & Down & solute carrier family 30 member 3 \\
\hline ILMN_1776157 & SEPTIN4 & -2.95971 & $1.55 \mathrm{E}-10$ & $8.61 \mathrm{E}-09$ & -8.46174 & Down & septin 4 \\
\hline ILMN_2401344 & PPP2R2C & -4.10878 & $1.58 \mathrm{E}-10$ & $8.76 \mathrm{E}-09$ & -8.45595 & Down & protein phosphatase 2 regulatory subunit Bgamma \\
\hline ILMN_1810533 & SLC6A15 & -2.50451 & $1.6 \mathrm{E}-10$ & $8.82 \mathrm{E}-09$ & -8.45248 & Down & solute carrier family 6 member 15 \\
\hline ILMN_2061565 & $\mathrm{PLCH} 2$ & -3.33408 & $1.64 \mathrm{E}-10$ & $9.02 \mathrm{E}-09$ & -8.4445 & Down & phospholipase $\mathrm{C}$ eta 2 \\
\hline ILMN_2320330 & MAL & -3.88298 & $1.7 \mathrm{E}-10$ & $9.27 \mathrm{E}-09$ & -8.43294 & Down & mal, T cell differentiation protein \\
\hline ILMN_1864900 & MIAT & -3.20549 & $1.85 \mathrm{E}-10$ & $9.99 \mathrm{E}-09$ & -8.40457 & Down & myocardial infarction associated transcript \\
\hline ILMN_1811820 & SRRM4 & -2.32735 & $1.91 \mathrm{E}-10$ & $1.03 \mathrm{E}-08$ & -8.3947 & Down & serine/arginine repetitive matrix 4 \\
\hline ILMN_1752299 & RAB6B & -2.38279 & $1.91 \mathrm{E}-10$ & $1.03 \mathrm{E}-08$ & -8.39417 & Down & RAB6B, member RAS oncogene family \\
\hline ILMN_1745134 & ANO4 & -2.40005 & $1.93 \mathrm{E}-10$ & $1.03 \mathrm{E}-08$ & -8.39109 & Down & anoctamin 4 \\
\hline ILMN_1805992 & SHTN1 & -2.86132 & $2.02 \mathrm{E}-10$ & $1.07 \mathrm{E}-08$ & -8.37671 & Down & shootin 1 \\
\hline ILMN_3310491 & MT-TF & -2.33202 & $2.11 \mathrm{E}-10$ & $1.11 \mathrm{E}-08$ & -8.36342 & Down & mitochondrially encoded tRNA-Phe (UUU/C) \\
\hline ILMN_1803818 & NMNAT2 & -2.34181 & $2.23 \mathrm{E}-10$ & $1.16 \mathrm{E}-08$ & -8.34533 & Down & nicotinamide nucleotide adenylyltransferase 2 \\
\hline ILMN_1768202 & ANKRD24 & -2.30127 & $2.24 \mathrm{E}-10$ & 1.17E-08 & -8.344 & Down & ankyrin repeat domain 24 \\
\hline ILMN_1659086 & NEFL & -2.94356 & $2.35 \mathrm{E}-10$ & $1.22 \mathrm{E}-08$ & -8.32821 & Down & $\begin{array}{l}\text { neurofilament light } \\
\text { gamma-aminobutyric acid type A receptor alpha2 }\end{array}$ \\
\hline ILMN_1659678 & GABRA2 & -2.81155 & $2.38 \mathrm{E}-10$ & $1.23 \mathrm{E}-08$ & -8.324 & Down & subunit \\
\hline ILMN_2395375 & GABBR1 & -2.53289 & 2.57E-10 & $1.3 \mathrm{E}-08$ & -8.30042 & Down & gamma-aminobutyric acid type $B$ receptor subunit 1 \\
\hline ILMN_2394366 & AMER2 & -3.10575 & $2.85 \mathrm{E}-10$ & $1.41 \mathrm{E}-08$ & -8.26771 & Down & APC membrane recruitment protein 2 \\
\hline ILMN_1662390 & ASPHD1 & -2.39581 & $2.85 \mathrm{E}-10$ & $1.41 \mathrm{E}-08$ & -8.26705 & Down & aspartate beta-hydroxylase domain containing 1 \\
\hline ILMN_1726388 & ACBD7 & -3.05718 & $2.9 \mathrm{E}-10$ & $1.43 \mathrm{E}-08$ & -8.26134 & Down & acyl-CoA binding domain containing 7 \\
\hline ILMN_1653836 & KIAA1549L & -2.81176 & $3.24 \mathrm{E}-10$ & $1.57 \mathrm{E}-08$ & -8.22672 & Down & KIAA1549 like \\
\hline ILMN_1773413 & DOCK9 & -2.26862 & $3.39 \mathrm{E}-10$ & $1.63 \mathrm{E}-08$ & -8.212 & Down & dedicator of cytokinesis 9 \\
\hline ILMN_2199768 & SLITRK4 & -2.62666 & $3.53 \mathrm{E}-10$ & $1.69 \mathrm{E}-08$ & -8.19922 & Down & SLIT and NTRK like family member 4 \\
\hline ILMN_2160428 & IL1RAPL1 & -3.21422 & $4.01 \mathrm{E}-10$ & $1.88 \mathrm{E}-08$ & -8.15842 & Down & interleukin 1 receptor accessory protein like 1 \\
\hline ILMN_2212999 & KIF5C & -2.56618 & $4.23 \mathrm{E}-10$ & $1.96 \mathrm{E}-08$ & -8.14147 & Down & kinesin family member $5 \mathrm{C}$ \\
\hline ILMN_2151114 & VSNL1 & -3.38849 & 4.63E-10 & $2.11 \mathrm{E}-08$ & -8.11336 & Down & visinin like 1 \\
\hline ILMN_1802082 & PRDM8 & -2.3624 & $5.01 \mathrm{E}-10$ & $2.26 \mathrm{E}-08$ & -8.0884 & Down & $\mathrm{PR} / \mathrm{SET}$ domain 8 \\
\hline ILMN_1906187 & CAMK1D & -2.37212 & 5.17E-10 & $2.33 \mathrm{E}-08$ & -8.07842 & Down & calcium/calmodulin dependent protein kinase ID \\
\hline ILMN_1733860 & SYNPR & -3.55772 & $5.22 \mathrm{E}-10$ & $2.35 \mathrm{E}-08$ & -8.07499 & Down & synaptoporin \\
\hline ILMN_1685834 & AMPH & -2.28773 & $5.26 \mathrm{E}-10$ & $2.36 \mathrm{E}-08$ & -8.07305 & Down & amphiphysin \\
\hline ILMN_1733366 & MAST1 & -2.31134 & $5.36 \mathrm{E}-10$ & $2.4 \mathrm{E}-08$ & -8.06693 & Down & microtubule associated serine/threonine kinase 1 \\
\hline ILMN_1733669 & $\mathrm{CDH} 18$ & -2.48672 & 5.57E-10 & 2.47E-08 & -8.05445 & Down & cadherin 18 \\
\hline ILMN_1653762 & NRSN1 & -3.19656 & $5.93 \mathrm{E}-10$ & $2.6 \mathrm{E}-08$ & -8.03477 & Down & neurensin 1 \\
\hline ILMN_1668891 & CHL1-AS2 & -2.34419 & $6.31 \mathrm{E}-10$ & $2.73 \mathrm{E}-08$ & -8.01522 & Down & CHL1 antisense RNA 2 \\
\hline ILMN_1733648 & SNAP91 & -3.95907 & $6.45 \mathrm{E}-10$ & $2.78 \mathrm{E}-08$ & -8.00847 & Down & synaptosome associated protein 91 \\
\hline ILMN_2134801 & ETNPPL & -3.70302 & $6.78 \mathrm{E}-10$ & $2.88 \mathrm{E}-08$ & -7.9927 & Down & ethanolamine-phosphate phospho-lyase \\
\hline ILMN_1658071 & ATP1B1 & -2.35521 & 7.27E-10 & $3.06 \mathrm{E}-08$ & -7.97053 & Down & ATPase $\mathrm{Na}+/ \mathrm{K}+$ transporting subunit beta 1 \\
\hline
\end{tabular}


medRxiv preprint doi: https://doi.org/10.1101/2020.12.21.20248616; this version posted December 23, 2020. The copyright holder for this preprint (which was not certified by peer review) is the author/funder, who has granted medRxiv a license to display the preprint in perpetuity.

All rights reserved. No reuse allowed without permission.

\begin{tabular}{|c|c|c|c|c|c|c|c|}
\hline ILMN_1761280 & NTSR2 & -2.62315 & $7.48 \mathrm{E}-10$ & $3.12 \mathrm{E}-08$ & -7.96157 & Down & neurotensin receptor 2 \\
\hline ILMN_1714600 & NAV3 & -2.40094 & $7.73 \mathrm{E}-10$ & $3.2 \mathrm{E}-08$ & -7.95114 & Down & neuron navigator 3 \\
\hline ILMN_2402172 & SEPTIN4 & -3.09006 & $7.82 \mathrm{E}-10$ & $3.23 \mathrm{E}-08$ & -7.94757 & Down & septin 4 \\
\hline ILMN_1671365 & PRODH & -2.48514 & $7.96 \mathrm{E}-10$ & $3.28 \mathrm{E}-08$ & -7.94203 & Down & proline dehydrogenase 1 \\
\hline ILMN_1733221 & SEMA4D & -3.43963 & $9.02 \mathrm{E}-10$ & $3.63 \mathrm{E}-08$ & -7.90272 & Down & $\begin{array}{l}\text { semaphorin 4D } \\
\text { calcium/calmodulin dependent protein kinase II }\end{array}$ \\
\hline ILMN_2315208 & САМK2B & -2.48818 & $9.65 \mathrm{E}-10$ & $3.84 \mathrm{E}-08$ & -7.88148 & Down & beta \\
\hline ILMN_1687533 & SEMA4D & -2.30286 & $9.79 \mathrm{E}-10$ & $3.87 \mathrm{E}-08$ & -7.87705 & Down & semaphorin 4D \\
\hline ILMN_1779241 & CRYM & -2.42211 & $9.93 \mathrm{E}-10$ & $3.92 \mathrm{E}-08$ & -7.87255 & Down & crystallin mu \\
\hline ILMN_1712719 & MAP7 & -2.87426 & $1.01 \mathrm{E}-09$ & 3.96E-08 & -7.86792 & Down & microtubule associated protein 7 \\
\hline ILMN_1747019 & PDYN & -2.53515 & $1.03 \mathrm{E}-09$ & $4.02 \mathrm{E}-08$ & -7.86151 & Down & prodynorphin \\
\hline ILMN_1741603 & BRINP1 & -2.8818 & $1.05 \mathrm{E}-09$ & 4.09E-08 & -7.85476 & Down & BMP/retinoic acid inducible neural specific 1 \\
\hline ILMN_3250273 & TMOD2 & -2.33828 & $1.06 \mathrm{E}-09$ & $4.11 \mathrm{E}-08$ & -7.85292 & Down & $\begin{array}{c}\text { tropomodulin } 2 \\
\text { potassium voltage-gated channel subfamily } 0\end{array}$ \\
\hline ILMN_1861270 & KCNQ3 & -2.29357 & $1.06 \mathrm{E}-09$ & $4.12 \mathrm{E}-08$ & -7.852 & Down & member 3 \\
\hline ILMN_1716803 & RALYL & -3.18533 & $1.09 \mathrm{E}-09$ & $4.19 \mathrm{E}-08$ & -7.84419 & Down & $\begin{array}{l}\text { RALY RNA binding protein like } \\
\text { glutamate ionotropic receptor NMDA type subunit }\end{array}$ \\
\hline ILMN_2227790 & GRIN2C & -2.56901 & $1.13 \mathrm{E}-09$ & $4.34 \mathrm{E}-08$ & -7.8323 & Down & $2 \mathrm{C}$ \\
\hline ILMN_1749493 & ADARB2 & -2.53028 & $1.14 \mathrm{E}-09$ & 4.37E-08 & -7.82971 & Down & adenosine deam inase RNA specific B2 (inactive) \\
\hline ILMN_1682459 & TUBB4A & -3.56359 & $1.15 \mathrm{E}-09$ & $4.4 \mathrm{E}-08$ & -7.82637 & Down & tubulin beta 4A class IVa \\
\hline ILMN_1745108 & ADAD2 & -2.46976 & $1.16 \mathrm{E}-09$ & 4.45E-08 & -7.82244 & Down & $\begin{array}{l}\text { adenosine deaminase domain containing } 2 \\
\text { ST8 alpha- } \mathrm{N} \text {-acetyl-neuraminide alpha-2,8- }\end{array}$ \\
\hline ILMN_1855910 & ST8SIA3 & -2.9129 & $1.19 \mathrm{E}-09$ & $4.52 \mathrm{E}-08$ & -7.81573 & Down & sialyltransferase 3 \\
\hline ILMN_1740555 & SNAP25 & -3.42015 & $1.24 \mathrm{E}-09$ & 4.66E-08 & -7.80272 & Down & synaptosome associated protein 25 \\
\hline ILMN_2394362 & AMER2 & -3.44799 & $1.27 \mathrm{E}-09$ & 4.75E-08 & -7.79473 & Down & APC membrane recruitment protein 2 \\
\hline ILMN_1882999 & AC107398.3 & -2.80213 & $1.28 \mathrm{E}-09$ & 4.76E-08 & -7.794 & Down & novel transcript, overlapping GABRB1 \\
\hline ILMN_1667381 & CAMKV & -4.07762 & $1.35 \mathrm{E}-09$ & 4.99E-08 & -7.77545 & Down & CaM kinase like vesicle associated \\
\hline ILMN_1748840 & CALB2 & -2.8963 & $1.42 \mathrm{E}-09$ & $5.2 \mathrm{E}-08$ & -7.76071 & Down & calbindin 2 \\
\hline ILMN_1709919 & PLP1 & -4.37455 & $1.5 \mathrm{E}-09$ & $5.45 \mathrm{E}-08$ & -7.74296 & Down & proteolipid protein 1 \\
\hline ILMN_1710553 & TMEM151A & -3.28416 & $1.51 \mathrm{E}-09$ & 5.49E-08 & -7.74018 & Down & $\begin{array}{l}\text { transmembrane protein } 151 \mathrm{~A} \\
\text { calcium/calmodulin dependent protein kinase II }\end{array}$ \\
\hline ILMN_1663397 & САМК2B & -2.30776 & $1.54 \mathrm{E}-09$ & 5.55E-08 & -7.73558 & Down & $\begin{array}{c}\text { beta } \\
\text { sodium/potassium transporting ATPase interacting }\end{array}$ \\
\hline ILMN_1671107 & NKAIN2 & -3.21052 & $1.55 \mathrm{E}-09$ & 5.57E-08 & -7.73327 & Down & 2 \\
\hline ILMN_1664047 & CACNA1E & -2.31076 & 1.57E-09 & 5.63E-08 & -7.72885 & Down & calcium voltage-gated channel subunit alpha1 E \\
\hline ILMN_3235379 & CALB2 & -2.68984 & $1.65 \mathrm{E}-09$ & 5.86E-08 & -7.71258 & Down & calbindin 2 \\
\hline ILMN_1661325 & PKP4 & -2.68314 & $1.73 \mathrm{E}-09$ & $6.09 \mathrm{E}-08$ & -7.69777 & Down & plakophilin 4 \\
\hline ILMN_1747627 & ABCA2 & -2.42989 & $2.02 \mathrm{E}-09$ & $6.94 \mathrm{E}-08$ & -7.6505 & Down & ATP binding cassette subfamily A member 2 \\
\hline ILMN_1654541 & ATP6V1G2 & -2.55124 & $2.03 \mathrm{E}-09$ & $6.94 \mathrm{E}-08$ & -7.64911 & Down & ATPase $\mathrm{H}+$ transporting $\mathrm{V} 1$ subunit $\mathrm{G} 2$ \\
\hline ILMN_2041236 & RAP1GAP2 & -2.53609 & $2.1 \mathrm{E}-09$ & 7.13E-08 & -7.63839 & Down & RAP1 GTPase activating protein 2 \\
\hline ILMN_3236825 & RAPGEF5 & -2.95772 & $2.16 \mathrm{E}-09$ & $7.3 \mathrm{E}-08$ & -7.62926 & Down & Rap guanine nucleotide exchange factor 5 \\
\hline ILMN_1709659 & TMEM151A & -3.1153 & $2.22 \mathrm{E}-09$ & $7.45 \mathrm{E}-08$ & -7.6208 & Down & transmembrane protein $151 \mathrm{~A}$ \\
\hline ILMN_1704210 & CNTNAP4 & -3.15629 & $2.41 \mathrm{E}-09$ & $7.98 \mathrm{E}-08$ & -7.59492 & Down & contactin associated protein like 4 \\
\hline ILMN_1814221 & NPTX1 & -3.72087 & $2.56 \mathrm{E}-09$ & $8.4 \mathrm{E}-08$ & -7.576 & Down & neuronal pentraxin 1 \\
\hline ILMN_1705253 & PTPRD & -2.60149 & $2.58 \mathrm{E}-09$ & 8.46E-08 & -7.57325 & Down & $\begin{array}{c}\text { protein tyrosine phosphatase receptor type } D \\
\text { phosphatidylinositol-5-phosphate 4-kinase type } 2\end{array}$ \\
\hline ILMN_3236637 & PIP4K2A & -2.56103 & $2.63 \mathrm{E}-09$ & 8.59E-08 & -7.56802 & Down & alpha \\
\hline ILMN_1768947 & MOBP & -4.40338 & $2.81 \mathrm{E}-09$ & 9.07E-08 & -7.54722 & Down & myelin associated oligodendrocyte basic protein \\
\hline ILMN_1694589 & PAQR8 & -2.5356 & $2.83 \mathrm{E}-09$ & $9.12 \mathrm{E}-08$ & -7.54534 & Down & and adipoQ \\
\hline
\end{tabular}


medRxiv preprint doi: https://doi.org/10.1101/2020.12.21.20248616; this version posted December 23, 2020. The copyright holder for this preprint (which was not certified by peer review) is the author/funder, who has granted medRxiv a license to display the preprint in perpetuity. All rights reserved. No reuse allowed without permission.

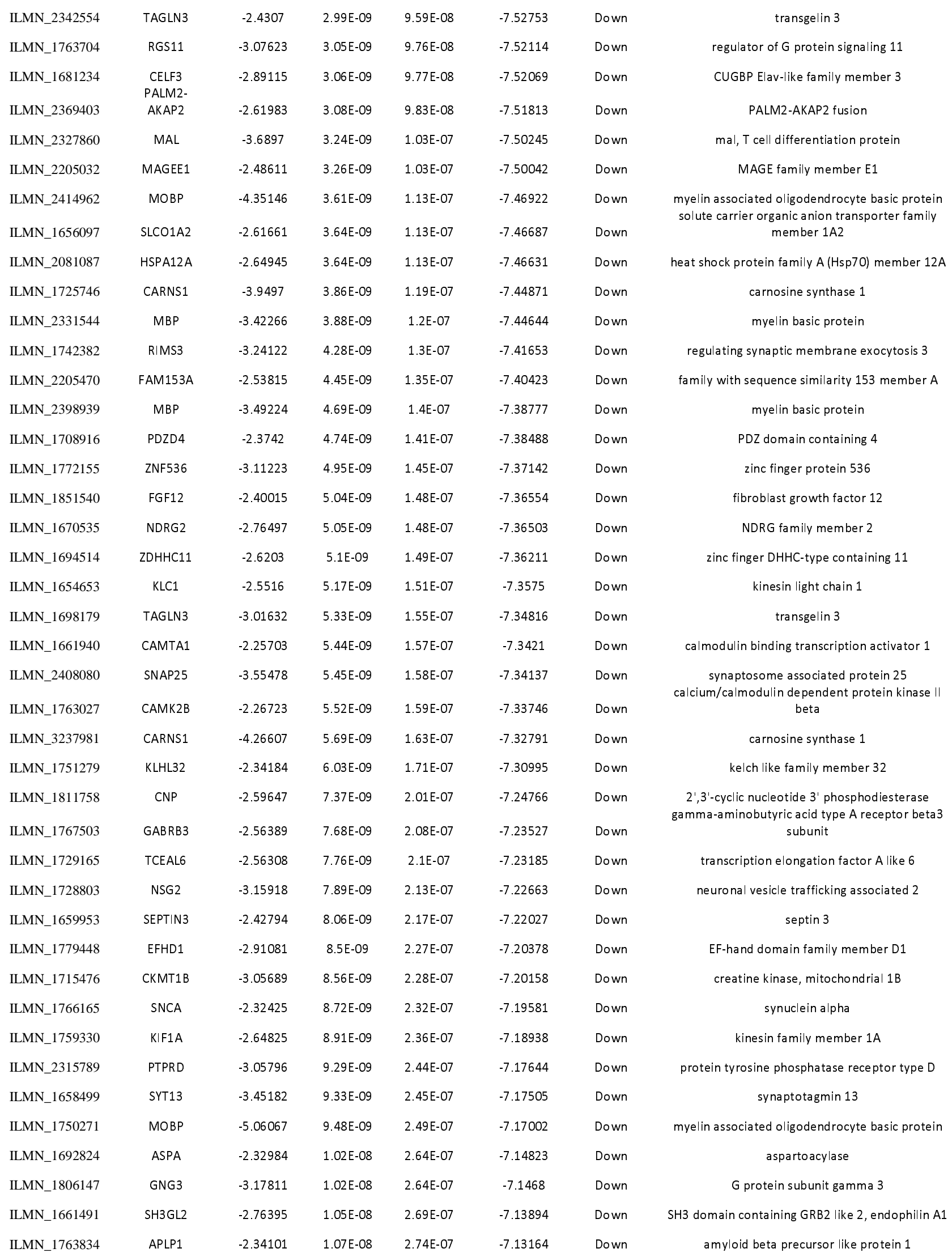


medRxiv preprint doi: https://doi.org/10.1101/2020.12.21.20248616; this version posted December 23, 2020. The copyright holder for this preprint (which was not certified by peer review) is the author/funder, who has granted medRxiv a license to display the preprint in perpetuity.

All rights reserved. No reuse allowed without permission.

\begin{tabular}{|c|c|c|c|c|c|c|c|}
\hline ILMN_2414135 & PLP1 & -3.95536 & $1.07 \mathrm{E}-08$ & $2.74 \mathrm{E}-07$ & -7.13145 & Down & $\begin{array}{c}\text { proteolipid protein } 1 \\
\text { potassium voltage-gated channel modifier subfamily }\end{array}$ \\
\hline ILMN_1761903 & KCNS1 & -2.70823 & $1.09 \mathrm{E}-08$ & $2.76 \mathrm{E}-07$ & -7.1283 & Down & $\begin{array}{l}\text { S member } 1 \\
\text { maturin, neural progenitor differentiation regulator }\end{array}$ \\
\hline ILMN_1672605 & MTURN & -2.5382 & $1.11 \mathrm{E}-08$ & $2.81 \mathrm{E}-07$ & -7.12161 & Down & $\begin{array}{l}\text { homolog } \\
\text { SPARC (osteonectin), cwcv and kazal like domains }\end{array}$ \\
\hline ILMN_1746013 & SPOCK1 & -2.3011 & $1.13 \mathrm{E}-08$ & $2.84 \mathrm{E}-07$ & -7.11707 & Down & proteoglycan 1 \\
\hline ILMN_1787673 & PLLP & -2.59248 & $1.19 \mathrm{E}-08$ & 2.97E-07 & -7.09893 & Down & plasmolipin \\
\hline ILMN_1733998 & DHRS9 & -2.8394 & $1.2 \mathrm{E}-08$ & $2.98 \mathrm{E}-07$ & -7.09655 & Down & $\begin{array}{c}\text { dehydrogenase/reductase } 9 \\
\text { gamma-aminobutyric acid type A receptor beta1 }\end{array}$ \\
\hline ILMN_2159730 & GABRB1 & -2.87824 & $1.23 \mathrm{E}-08$ & $3.04 \mathrm{E}-07$ & -7.08959 & Down & subunit \\
\hline ILMN_2221808 & DGCR5 & -2.26606 & $1.26 \mathrm{E}-08$ & $3.12 \mathrm{E}-07$ & -7.08171 & Down & DiGeorge syndrome critical region gene 5 \\
\hline ILMN_2120555 & ADCY1 & -2.77672 & $1.31 \mathrm{E}-08$ & $3.22 \mathrm{E}-07$ & -7.06977 & Down & adenylatecyclase 1 \\
\hline ILMN_1814333 & SERPINI1 & -2.89983 & $1.35 \mathrm{E}-08$ & $3.3 \mathrm{E}-07$ & -7.06144 & Down & serpin family I member 1 \\
\hline ILMN_1685194 & CLDN10 & -2.28618 & $1.39 \mathrm{E}-08$ & 3.39E-07 & -7.05178 & Down & $\begin{array}{l}\qquad \text { claudin } 10 \\
\text { potassium inwardly rectifying channel subfamily J }\end{array}$ \\
\hline ILMN_1805376 & KCNJ6 & -2.28288 & $1.47 \mathrm{E}-08$ & $3.55 \mathrm{E}-07$ & -7.03554 & Down & $\begin{array}{c}\text { member } 6 \\
\text { calcium/calmodulin dependent protein kinase II }\end{array}$ \\
\hline ILMN_2376194 & CAMK2B & -3.01022 & $1.47 \mathrm{E}-08$ & $3.57 \mathrm{E}-07$ & -7.03379 & Down & beta \\
\hline ILMN_1801476 & CDS1 & -2.26248 & $1.49 \mathrm{E}-08$ & $3.61 \mathrm{E}-07$ & -7.02975 & Down & CDP-diacylglycerol synthase 1 \\
\hline ILMN_1679176 & CLCA4 & -2.5965 & $1.58 \mathrm{E}-08$ & $3.78 \mathrm{E}-07$ & -7.01166 & Down & chloride channel accessory 4 \\
\hline ILMN_1776516 & ITPKA & -2.4544 & $1.62 \mathrm{E}-08$ & $3.87 \mathrm{E}-07$ & -7.00399 & Down & inositol-trisphosphate 3-kinase A \\
\hline ILMN_2149164 & SFRP1 & -2.67363 & $1.66 \mathrm{E}-08$ & $3.94 \mathrm{E}-07$ & -6.99664 & Down & secreted frizzled related protein 1 \\
\hline ILMN_2073184 & S1PR5 & -2.80163 & $1.71 \mathrm{E}-08$ & 4.03E-07 & -6.98772 & Down & sphingosine-1-phosphate receptor 5 \\
\hline ILMN_1685709 & TMEM125 & -3.262 & $1.89 \mathrm{E}-08$ & $4.37 \mathrm{E}-07$ & -6.95699 & Down & transmembrane protein 125 \\
\hline ILMN_1905482 & GPR37 & -3.09976 & $1.96 \mathrm{E}-08$ & 4.49E-07 & -6.94686 & Down & $\begin{array}{l}\text { G protein-coupled receptor } 37 \\
\text { pleckstrin homology, MyTH4 and FERM domain }\end{array}$ \\
\hline ILMN_1699254 & PLEKHH1 & -3.00817 & $1.98 \mathrm{E}-08$ & $4.53 \mathrm{E}-07$ & -6.94257 & Down & containing $\mathrm{H} 1$ \\
\hline ILMN_1825369 & NETO1 & -3.10083 & $2.25 \mathrm{E}-08$ & 5.04E-07 & -6.90344 & Down & neuropilin and tolloid like 1 \\
\hline ILMN_1756755 & LINGO1 & -2.2588 & $2.56 \mathrm{E}-08$ & $5.61 \mathrm{E}-07$ & -6.86444 & Down & leucine rich repeat and Ig domain containing 1 \\
\hline ILMN_1745817 & NELL1 & -2.46964 & $2.66 \mathrm{E}-08$ & 5.77E-07 & -6.85242 & Down & $\begin{array}{l}\text { neural EGFL like } 1 \\
\text { gamma-aminobutyric acid type A receptor gamma2 }\end{array}$ \\
\hline ILMN_1800270 & GABRG2 & -2.78306 & $2.74 \mathrm{E}-08$ & $5.92 \mathrm{E}-07$ & -6.84305 & Down & subunit \\
\hline ILMN_2380938 & SYT7 & -3.0799 & $3.36 \mathrm{E}-08$ & 7.02E-07 & -6.78062 & Down & synaptotagmin 7 \\
\hline ILMN_1691355 & HHATL & -2.62742 & $3.38 \mathrm{E}-08$ & 7.04E-07 & -6.77908 & Down & hedgehog acyltransferase like \\
\hline ILMN_1681087 & SLC7A10 & -2.4568 & $3.39 \mathrm{E}-08$ & 7.06E-07 & -6.77814 & Down & solute carrier family 7 member 10 \\
\hline ILMN_1791531 & $\mathrm{FA} 2 \mathrm{H}$ & -2.69324 & $3.39 \mathrm{E}-08$ & 7.06E-07 & -6.77779 & Down & $\begin{array}{l}\text { fatty acid 2-hydroxylase } \\
\text { pleckstrin homology and RhoGEF domain containing }\end{array}$ \\
\hline ILMN_1780671 & PLEKHG3 & -2.27187 & $3.4 \mathrm{E}-08$ & 7.07E-07 & -6.77734 & Down & G3 \\
\hline ILMN_1744191 & SLC6A1 & -2.63638 & $3.49 \mathrm{E}-08$ & $7.23 \mathrm{E}-07$ & -6.76897 & Down & solute carrier family 6 member 1 \\
\hline ILMN_1664464 & PTGDS & -2.96706 & $3.91 \mathrm{E}-08$ & $7.9 \mathrm{E}-07$ & -6.73424 & Down & prostaglandin D2 synthase \\
\hline ILMN_1737631 & PAQR6 & -3.01575 & $4.11 \mathrm{E}-08$ & $8.24 \mathrm{E}-07$ & -6.71883 & Down & progestin and adipo $Q$ receptor family member 6 \\
\hline ILMN_1680928 & DNM3 & -2.62285 & $4.28 \mathrm{E}-08$ & $8.52 \mathrm{E}-07$ & -6.70675 & Down & dynamin 3 \\
\hline ILMN_1701933 & SNCA & -2.4264 & 4.33E-08 & $8.61 \mathrm{E}-07$ & -6.70329 & Down & synuclein alpha \\
\hline ILMN_2330382 & PAQR6 & -2.91794 & $4.4 \mathrm{E}-08$ & $8.73 \mathrm{E}-07$ & -6.69789 & Down & progestin and adipoQ receptor family member 6 \\
\hline ILMN_2298464 & MOBP & -4.02241 & 4.69E-08 & $9.17 \mathrm{E}-07$ & -6.67835 & Down & myelin associated oligodendrocyte basic protein \\
\hline ILMN_1739235 & OMG & -2.8059 & $5.3 \mathrm{E}-08$ & $1.02 \mathrm{E}-06$ & -6.64113 & Down & oligodendrocyte myelin glycoprotein \\
\hline ILMN_1714709 & OLFM1 & -2.66656 & $5.77 \mathrm{E}-08$ & 1.09E-06 & -6.6153 & Down & olfactomedin 1 \\
\hline ILMN_1778924 & PDE1A & -2.46891 & 6.41E-08 & 1.19E-06 & -6.58271 & Down & phosphodiesterase $1 \mathrm{~A}$ \\
\hline ILMN_1848916 & TTLL7 & -2.29241 & $7 E-08$ & $1.29 \mathrm{E}-06$ & -6.55578 & Down & tubulin tyrosine ligase like 7 \\
\hline
\end{tabular}


medRxiv preprint doi: https://doi.org/10.1101/2020.12.21.20248616; this version posted December 23, 2020. The copyright holder for this preprint (which was not certified by peer review) is the author/funder, who has granted medRxiv a license to display the preprint in perpetuity.

All rights reserved. No reuse allowed without permission.

\begin{tabular}{|c|c|c|c|c|c|c|c|}
\hline ILMN_1733042 & BCAS1 & -3.57087 & $7.22 \mathrm{E}-08$ & $1.33 \mathrm{E}-06$ & -6.54658 & Down & breast carcinoma amplified sequence 1 \\
\hline ILMN_1669410 & CHGA & -2.59314 & $7.71 \mathrm{E}-08$ & $1.4 \mathrm{E}-06$ & -6.52654 & Down & chromogranin A \\
\hline ILMN_1732066 & CKMT1A & -2.60974 & $7.84 \mathrm{E}-08$ & $1.42 \mathrm{E}-06$ & -6.52119 & Down & creatine kinase, mitochondrial $1 \mathrm{~A}$ \\
\hline ILMN_3242623 & COPG2IT1 & -2.35434 & $8.18 \mathrm{E}-08$ & $1.48 \mathrm{E}-06$ & -6.50846 & Down & COPG2 imprinted transcript 1 \\
\hline ILMN_1749410 & PKP4 & -2.38187 & $8.47 \mathrm{E}-08$ & $1.52 \mathrm{E}-06$ & -6.49766 & Down & plakophilin 4 \\
\hline ILMN_1730928 & CDK5R1 & -2.31611 & $8.52 \mathrm{E}-08$ & $1.53 \mathrm{E}-06$ & -6.49601 & Down & cyclin dependent kinase 5 regulatory subunit 1 \\
\hline ILMN_1656560 & PARM1 & -2.28534 & $8.54 \mathrm{E}-08$ & $1.53 \mathrm{E}-06$ & -6.4953 & Down & prostate androgen-regulated mucin-like protein 1 \\
\hline ILMN_1800642 & RELN & -2.59448 & $8.92 \mathrm{E}-08$ & $1.59 \mathrm{E}-06$ & -6.48174 & Down & reelin \\
\hline ILMN_1776519 & RAP1GAP & -2.30778 & $8.95 \mathrm{E}-08$ & 1.59E-06 & -6.48098 & Down & RAP1 GTPase activating protein \\
\hline ILMN_2082865 & PLLP & -2.7193 & $9.45 \mathrm{E}-08$ & $1.66 \mathrm{E}-06$ & -6.46437 & Down & $\begin{array}{l}\text { plasmolipin } \\
\text { internexin neuronal intermediate filament protein }\end{array}$ \\
\hline ILMN_1673704 & INA & -3.33745 & $9.76 \mathrm{E}-08$ & $1.71 \mathrm{E}-06$ & -6.45439 & Down & $\begin{array}{c}\text { alpha } \\
\text { phospholysinephosphohistidine inorganic }\end{array}$ \\
\hline ILMN_1752199 & LHPP & -2.73892 & $9.83 \mathrm{E}-08$ & $1.72 \mathrm{E}-06$ & -6.45217 & Down & pyrophosphate phosphatase \\
\hline ILMN_2216815 & MAP7 & -2.29759 & $1.07 \mathrm{E}-07$ & 1.84E-06 & -6.42597 & Down & microtubule associated protein 7 \\
\hline ILMN_1712913 & UNC5A & -2.9749 & $1.19 \mathrm{E}-07$ & $2.01 \mathrm{E}-06$ & -6.39463 & Down & unc- 5 netrin receptor $A$ \\
\hline ILMN_1676504 & RPRML & -2.98163 & $1.23 \mathrm{E}-07$ & 2.07E-06 & -6.38414 & Down & reprimo like \\
\hline ILMN_1786989 & NKX6-2 & -3.55497 & $1.25 \mathrm{E}-07$ & $2.1 \mathrm{E}-06$ & -6.37861 & Down & NK6 homeobox 2 \\
\hline ILMN_1783231 & PLEKHB1 & -2.42662 & $1.26 \mathrm{E}-07$ & $2.12 \mathrm{E}-06$ & -6.37547 & Down & pleckstrin homology domain containing B1 \\
\hline ILMN_1688464 & MAP6D1 & -2.33871 & 1.31E-07 & $2.18 \mathrm{E}-06$ & -6.36507 & Down & MAP6 domain containing 1 \\
\hline ILMN_1718896 & CNTN2 & -3.33954 & 1.37E-07 & $2.26 \mathrm{E}-06$ & -6.35111 & Down & contactin 2 \\
\hline ILMN_1741021 & $\mathrm{CH} 25 \mathrm{H}$ & -2.45248 & $1.38 \mathrm{E}-07$ & $2.27 \mathrm{E}-06$ & -6.34928 & Down & cholesterol 25-hydroxylase \\
\hline ILMN_1756807 & GPR62 & -2.31001 & $1.41 \mathrm{E}-07$ & $2.32 \mathrm{E}-06$ & -6.34155 & Down & G protein-coupled receptor 62 \\
\hline ILMN_1785380 & SLC1A2 & -2.46557 & $1.48 \mathrm{E}-07$ & $2.41 \mathrm{E}-06$ & -6.32766 & Down & $\begin{array}{l}\text { solute carrier family } 1 \text { member } 2 \\
\text { ectonucleotidepyrophosphatase/phosphodiesterase }\end{array}$ \\
\hline ILMN_2373791 & ENPP2 & -3.41955 & $1.58 \mathrm{E}-07$ & $2.54 \mathrm{E}-06$ & -6.30782 & Down & 2 \\
\hline ILMN_1803773 & MAG & -3.89187 & $1.62 \mathrm{E}-07$ & $2.59 \mathrm{E}-06$ & -6.29979 & Down & myelin associated glycoprotein \\
\hline ILMN_1752668 & DAAM2 & -2.61789 & 1.81E-07 & $2.85 \mathrm{E}-06$ & -6.26532 & Down & dishevelled associated activator of morphogenesis 2 \\
\hline ILMN_2361603 & NDRG2 & -2.57271 & $1.86 \mathrm{E}-07$ & $2.92 \mathrm{E}-06$ & -6.25682 & Down & NDRG family member 2 \\
\hline ILMN_1780255 & KLK6 & -3.88547 & $2.02 \mathrm{E}-07$ & $3.12 \mathrm{E}-06$ & -6.23208 & Down & kallikrein related peptidase 6 \\
\hline ILMN_2332691 & CAPN3 & -2.5065 & $2.18 \mathrm{E}-07$ & 3.33E-06 & -6.20858 & Down & calpain 3 \\
\hline ILMN_1713422 & ST18 & -2.77258 & $2.28 \mathrm{E}-07$ & $3.46 \mathrm{E}-06$ & -6.19509 & Down & ST18 C2H2C-type zinc finger transcription factor \\
\hline ILMN_2310001 & MOG & -2.83708 & $2.34 \mathrm{E}-07$ & 3.53E-06 & -6.18722 & Down & myelin oligodendrocyte glycoprotein \\
\hline ILMN_1685496 & RGS7 & -2.41034 & $2.61 \mathrm{E}-07$ & $3.88 \mathrm{E}-06$ & -6.15391 & Down & $\begin{array}{l}\text { regulator of } G \text { protein signaling } 7 \\
\text { glycosylphosphatidylinositol anchored high density }\end{array}$ \\
\hline ILMN_1726210 & GPIHBP1 & -2.62873 & $2.63 \mathrm{E}-07$ & $3.91 \mathrm{E}-06$ & -6.15132 & Down & lipoprotein binding protein 1 \\
\hline ILMN_1887357 & CHRM3 & -2.84305 & $2.64 \mathrm{E}-07$ & $3.92 \mathrm{E}-06$ & -6.1502 & Down & $\begin{array}{l}\text { cholinergic receptor muscarinic } 3 \\
\text { sodium/potassium transporting ATPase interacting }\end{array}$ \\
\hline ILMN_3249603 & NKAIN2 & -2.88405 & $2.68 \mathrm{E}-07$ & 3.97E-06 & -6.146 & Down & ectonucleotidepyrophosphatase/phosphodiesterase \\
\hline ILMN_1780799 & ENPP2 & -3.32961 & $2.91 \mathrm{E}-07$ & 4.25E-06 & -6.12029 & Down & 2 \\
\hline ILMN_2136147 & BCAS1 & -3.52959 & 3.54E-07 & $5.05 \mathrm{E}-06$ & -6.06104 & Down & breast carcinoma amplified sequence 1 \\
\hline ILMN_1811437 & MYRF & -2.46785 & $3.6 \mathrm{E}-07$ & $5.12 \mathrm{E}-06$ & -6.05623 & Down & myelin regulatory factor \\
\hline ILMN_1756928 & RTN1 & -2.50887 & 4.37E-07 & $6.03 \mathrm{E}-06$ & -5.9966 & Down & reticulon 1 \\
\hline ILMN_2281786 & RTN1 & -3.0012 & $4.5 \mathrm{E}-07$ & $6.17 \mathrm{E}-06$ & -5.98795 & Down & reticulon 1 \\
\hline ILMN_1768705 & SYT4 & -3.47955 & $4.59 \mathrm{E}-07$ & $6.28 \mathrm{E}-06$ & -5.98198 & Down & synaptotagmin 4 \\
\hline ILMN_1708110 & TMEM144 & -2.55614 & $4.86 \mathrm{E}-07$ & $6.58 \mathrm{E}-06$ & -5.96442 & Down & transmembrane protein 144 \\
\hline ILMN_1687971 & CAPN3 & -2.79283 & 5.31E-07 & 7.11E-06 & -5.93722 & Down & calpain 3 \\
\hline
\end{tabular}


medRxiv preprint doi: https://doi.org/10.1101/2020.12.21.20248616; this version posted December 23, 2020. The copyright holder for this preprint (which was not certified by peer review) is the author/funder, who has granted medRxiv a license to display the preprint in perpetuity.

All rights reserved. No reuse allowed without permission.

\begin{tabular}{|c|c|c|c|c|c|c|c|}
\hline ILMN_2292646 & GAD1 & -2.88848 & $6.21 \mathrm{E}-07$ & $8.13 E-06$ & -5.88947 & Down & glutamate decarboxylase 1 \\
\hline ILMN_1675331 & PEG3 & -2.53882 & $6.32 \mathrm{E}-07$ & $8.25 \mathrm{E}-06$ & -5.88397 & Down & $\begin{array}{c}\text { paternally expressed } 3 \\
\text { gamma-aminobutyric acid type A receptor beta3 }\end{array}$ \\
\hline ILMN_1709681 & GABRB3 & -2.32593 & 7.56E-07 & $9.6 \mathrm{E}-06$ & -5.82934 & Down & subunit \\
\hline ILMN_1768425 & TF & -3.16473 & $8.81 \mathrm{E}-07$ & $1.09 \mathrm{E}-05$ & -5.78244 & Down & transferrin \\
\hline ILMN_1905546 & $\# \mathrm{~N} / \mathrm{A}$ & -2.51858 & $9.18 \mathrm{E}-07$ & 1.13E-05 & -5.76999 & Down & NA \\
\hline ILMN_1682123 & TतC9B & -2.29827 & $9.23 \mathrm{E}-07$ & $1.14 \mathrm{E}-05$ & -5.76838 & Down & tetratricopeptide repeat domain 9B \\
\hline ILMN_2320164 & P2RY12 & -2.66116 & $9.79 E-07$ & $1.2 \mathrm{E}-05$ & -5.75028 & Down & purinergic receptor $\mathrm{P} 2 \mathrm{Y} 12$ \\
\hline ILMN_1723048 & GJC2 & -2.57128 & $1.07 \mathrm{E}-06$ & $1.3 \mathrm{E}-05$ & -5.722 & Down & gap junction protein gamma 2 \\
\hline ILMN_1723123 & FGFR3 & -2.46836 & $1.15 \mathrm{E}-06$ & $1.37 \mathrm{E}-05$ & -5.70055 & Down & fibroblast growth factor receptor 3 \\
\hline ILMN_1671149 & MEG3 & -2.40804 & $1.21 \mathrm{E}-06$ & $1.43 \mathrm{E}-05$ & -5.68565 & Down & maternally expressed 3 \\
\hline ILMN_1758067 & RGS4 & -2.45357 & $1.48 \mathrm{E}-06$ & $1.7 \mathrm{E}-05$ & -5.62372 & Down & regulator of $\mathrm{G}$ protein signaling 4 \\
\hline ILMN_1660718 & GABBR2 & -2.7931 & $1.61 \mathrm{E}-06$ & $1.83 \mathrm{E}-05$ & -5.5979 & Down & $\begin{array}{l}\text { gamma-aminobutyric acid type } B \text { receptor subunit } 2 \\
\text { potassium two pore domain channel subfamily } \mathrm{K}\end{array}$ \\
\hline ILMN_1711988 & KCNK12 & -2.63599 & $1.62 \mathrm{E}-06$ & $1.84 \mathrm{E}-05$ & -5.59544 & Down & member 12 \\
\hline ILMN_1678618 & ELAVL3 & -2.31121 & $1.71 \mathrm{E}-06$ & $1.92 \mathrm{E}-05$ & -5.5801 & Down & ELAV like RNA binding protein 3 \\
\hline ILMN_1731397 & STMN4 & -2.85752 & $1.88 \mathrm{E}-06$ & $2.09 \mathrm{E}-05$ & -5.55086 & Down & stathmin 4 \\
\hline ILMN_1741698 & OPCML & -2.72681 & $2.1 \mathrm{E}-06$ & $2.3 \mathrm{E}-05$ & -5.51714 & Down & opioid binding protein/cell adhesion molecule like \\
\hline ILMN_1813295 & LMO3 & -2.74695 & $2.86 \mathrm{E}-06$ & $2.98 \mathrm{E}-05$ & -5.42211 & Down & LIM domain only 3 \\
\hline ILMN_1766499 & HSPA2 & -2.46921 & $3.25 \mathrm{E}-06$ & $3.32 \mathrm{E}-05$ & -5.3824 & Down & $\begin{array}{l}\text { heat shock protein family A (Hsp } 70) \text { member } 2 \\
\text { lysosomal associated membrane protein family }\end{array}$ \\
\hline ILMN_1713561 & LAMP5 & -2.40514 & $3.44 \mathrm{E}-06$ & 3.49E-05 & -5.36529 & Down & member 5 \\
\hline ILMN_1694653 & CNDP1 & -2.62109 & $3.77 \mathrm{E}-06$ & $3.77 \mathrm{E}-05$ & -5.33662 & Down & carnosinedipeptidase 1 \\
\hline ILMN_1690397 & DYNC1I1 & -2.28352 & $3.9 \mathrm{E}-06$ & 3.87E-05 & -5.32688 & Down & dynein cytoplasmic 1 intermediate chain 1 \\
\hline ILMN_2120210 & RCAN2 & -2.31108 & $3.99 \mathrm{E}-06$ & 3.96E-05 & -5.3191 & Down & regulator of calcineurin 2 \\
\hline ILMN_1758816 & UGT8 & -2.51259 & 4.65E-06 & 4.5E-05 & -5.27241 & Down & UDP glycosyltransferase 8 \\
\hline ILMN_1718949 & SLC39A12 & -2.36133 & $6 \mathrm{E}-06$ & $5.63 \mathrm{E}-05$ & -5.19383 & Down & solute carrier family 39 mem ber 12 \\
\hline ILMN_2126038 & STMN2 & -2.97818 & $8.21 \mathrm{E}-06$ & 7.34E-05 & -5.09678 & Down & stathmin 2 \\
\hline ILMN_1731062 & NPY & -3.02278 & $1.02 \mathrm{E}-05$ & $8.8 \mathrm{E}-05$ & -5.02894 & Down & neuropeptide $Y$ \\
\hline ILMN_1682298 & ERMN & -2.65514 & $1.05 \mathrm{E}-05$ & $9 E-05$ & -5.02061 & Down & ermin \\
\hline ILMN_1699585 & BHLHE22 & -2.30366 & $1.17 \mathrm{E}-05$ & $9.84 \mathrm{E}-05$ & -4.98788 & Down & basic helix-loop-helix family mem ber e22 \\
\hline ILMN_1751346 & ERBB3 & -2.26009 & $1.45 \mathrm{E}-05$ & 0.000118 & -4.92092 & Down & $\begin{array}{l}\text { erb-b2 receptor tyrosine kinase } 3 \\
\text { transmembrane protein with EGF like and two }\end{array}$ \\
\hline ILMN_1730645 & TMEFF2 & -2.65258 & $1.56 \mathrm{E}-05$ & 0.000127 & -4.89649 & Down & follistatin like domains 2 \\
\hline ILMN_3235832 & CCL4L2 & -2.30764 & $1.66 \mathrm{E}-05$ & 0.000133 & -4.87842 & Down & C-C motif chemokine ligand 4 like 2 \\
\hline ILMN_1790106 & PLP1 & -2.2646 & $2.72 \mathrm{E}-05$ & 0.000205 & -4.72271 & Down & proteolipid protein 1 \\
\hline ILMN_1780170 & APOD & -2.29182 & 3.09E-05 & 0.000227 & -4.68327 & Down & apolipoprotein D \\
\hline ILMN_2061435 & MEG3 & -2.6632 & $3.2 \mathrm{E}-05$ & 0.000234 & -4.67176 & Down & maternally expressed 3 \\
\hline ILMN_1795679 & STMN2 & -2.83979 & $6.36 \mathrm{E}-05$ & 0.000424 & -4.45418 & Down & stathmin 2 \\
\hline ILMN_1653750 & SOX10 & -2.3027 & $8.05 \mathrm{E}-05$ & 0.000519 & -4.37883 & Down & $\begin{array}{l}\text { SRY-box transcription factor } 10 \\
\text { FosB proto-oncogene, AP-1 transcription factor }\end{array}$ \\
\hline ILMN_1751607 & FOSB & -2.4652 & $8.3 \mathrm{E}-05$ & 0.000532 & -4.36908 & Down & subunit \\
\hline ILMN_1765966 & CHGB & -2.41365 & $9.13 \mathrm{E}-05$ & 0.000577 & -4.3383 & Down & chromogranin B \\
\hline ILMN_1764573 & XIST & -2.59683 & $\begin{array}{l}0.00027 \\
0.00457\end{array}$ & 0.001461 & -3.9858 & Down & $\mathrm{X}$ inactive specific transcript \\
\hline ILMN_1691413 & NNAT & -2.45885 & 6 & 0.016193 & -3.00004 & Down & neuronatin \\
\hline
\end{tabular}


medRxiv preprint doi: https://doi.org/10.1101/2020.12.21.20248616; this version posted December 23, 2020. The copyright holder for this preprint (which was not certified by peer review) is the author/funder, who has granted medRxiv a license to display the preprint in perpetuity. All rights reserved. No reuse allowed without permission.

Table 2 The enriched pathway terms of the up regulated differentially expressed genes

BIOCYC

\begin{tabular}{|c|c|c|c|c|c|c|c|}
\hline Pathway ID & Pathway Name & P-value & FDR B\&H & $\begin{array}{l}\text { FDR } \\
\text { B\&Y }\end{array}$ & Bonferroni & $\begin{array}{l}\text { Gene } \\
\text { Count }\end{array}$ & Gene \\
\hline 1108784 & $\begin{array}{c}\text { reactive oxygen } \\
\text { species } \\
\text { degradation }\end{array}$ & $1.41 \mathrm{E}-03$ & $3.53 \mathrm{E}-02$ & $1.35 \mathrm{E}-01$ & $3.53 \mathrm{E}-02$ & 3 & GPX8,SOD2,GPX7 \\
\hline 142235 & $\begin{array}{l}\text { glutamate removal } \\
\text { from folates }\end{array}$ & $2.44 \mathrm{E}-02$ & $2.03 \mathrm{E}-01$ & 7.75E-01 & $6.09 \mathrm{E}-01$ & 1 & GGH \\
\hline 142290 & $\begin{array}{c}\text { spermine } \\
\text { biosynthesis }\end{array}$ & $2.44 \mathrm{E}-02$ & $2.03 \mathrm{E}-01$ & 7.75E-01 & $6.09 \mathrm{E}-01$ & 1 & SMS \\
\hline 142427 & $\begin{array}{l}\text { sulfate activation } \\
\text { for sulfonation }\end{array}$ & 4.70E-02 & $2.94 \mathrm{E}-01$ & $1.00 \mathrm{E}+00$ & $1.00 \mathrm{E}+00$ & 1 & PAPSS1 \\
\hline 142214 & NAD salvage & $6.97 \mathrm{E}-02$ & $2.98 \mathrm{E}-01$ & $1.00 \mathrm{E}+00$ & $1.00 \mathrm{E}+00$ & 1 & NAMPT \\
\hline 545346 & heme degradation & $9.18 \mathrm{E}-02$ & $2.98 \mathrm{E}-01$ & $1.00 \mathrm{E}+00$ & $1.00 \mathrm{E}+00$ & 1 & HMOX1 \\
\hline 142304 & $\begin{array}{l}\text { UDP-N-acetyl-D- } \\
\text { glucosamine } \\
\text { biosynthesis II }\end{array}$ & $1.13 \mathrm{E}-01$ & $2.98 \mathrm{E}-01$ & $1.00 \mathrm{E}+00$ & $1.00 \mathrm{E}+00$ & 1 & UAP1 \\
\hline 142257 & $\begin{array}{l}\text { phosphatidylcholi } \\
\text { ne biosynthesis }\end{array}$ & $1.35 \mathrm{E}-01$ & $2.98 \mathrm{E}-01$ & $1.00 \mathrm{E}+00$ & $1.00 \mathrm{E}+00$ & 1 & CHPT1 \\
\hline 545317 & $\begin{array}{c}\text { zymosterol } \\
\text { biosynthesis }\end{array}$ & $1.35 \mathrm{E}-01$ & $2.98 \mathrm{E}-01$ & $1.00 \mathrm{E}+00$ & $1.00 \mathrm{E}+00$ & 1 & CYP51A1 \\
\hline 545299 & $\begin{array}{l}\text { C20 prostanoid } \\
\text { biosynthesis }\end{array}$ & $1.55 \mathrm{E}-01$ & $2.98 \mathrm{E}-01$ & $1.00 \mathrm{E}+00$ & $1.00 \mathrm{E}+00$ & 1 & PTGES3 \\
\hline
\end{tabular}

\section{KEGG}

PTTG1,TTK,CDC4

5,WEE1,MYC,BUB 1,E2F2,CCNA2,CC NB1,CCNB2,CDK1 ,MAD2L1,PCNA,C DK2 
medRxiv preprint doi: https://doi.org/10.1101/2020.12.21.20248616; this version posted December 23, 2020. The copyright holder for this preprint (which was not certified by peer review) is the author/funder, who has granted medRxiv a license to display the preprint in perpetuity. All rights reserved. No reuse allowed without permission.

interaction

83073

695200

83105

153910

177876

812256

132956

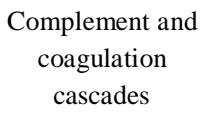

Pathways in cancer

Phagosome

RNA transport

TNF signaling pathway

Metabolic pathways

$$
5.61 \mathrm{E}-04
$$

1.03E-02

.35E-02

1.52E-01

9.04E-01

$1.00 \mathrm{E}+00$

1.61E-01

7.17E-01

$1.00 \mathrm{E}+00$

$1.00 \mathrm{E}+00$

4.76E-01

9.76E-01

9.91E-01
OL6A3,TNC,ITGA 5,LAMA4,LAMB1, LAMC1,CD44

A2M,F2R,F3,F13A

1,PLAU,CFI,C1S,T

FPI

IF1A,HMOX1,VE

GFA,ANGPT2,GA PDH,TIMP1,EIF4E

HIF1 A,ETS1,F2R,C KS2,MMP9, VEGF A,COL4A1,BIRC5, GNA13,CXCL8,M YC,GNG10,LAMA 4,LAMB1,LAMC1, E2F2,CDK2

HLA-A,HLAG,ITGA5,TUBB6,T UBA1C,SEC61G

SUMO2,NXT2,RA N,EEF1A1,EIF1AY ,EIF4E

CCL20,MMP9,VC AM1

RPN2,HMOX1,LIP G,AK4,UQCRH,A MY1A,SMS,PTGE S3,UAP1,GAPDH, MTHFD2,CYP51A 1,GBE1,CHPT1,PY GL,NNMT,RDH10, PAPSS1,NAMPT,P 4HA1,TPI

Pathway Interaction Database

\begin{tabular}{|c|c|c|c|c|c|c|c|}
\hline 137935 & $\begin{array}{c}\text { FOXM1 } \\
\text { transcription factor } \\
\text { network }\end{array}$ & $5.63 \mathrm{E}-10$ & $5.74 \mathrm{E}-08$ & $2.99 \mathrm{E}-07$ & $5.74 \mathrm{E}-08$ & 11 & $\begin{array}{c}\text { CENPA,CENPF,BI } \\
\text { RC5,MYC,NEK2,L } \\
\text { AMA4,CCNB1,CC } \\
\text { NB2,CDK1,CDK2, } \\
\text { AURKB }\end{array}$ \\
\hline 138007 & $\begin{array}{c}\text { PLK1 signaling } \\
\text { events }\end{array}$ & $6.02 \mathrm{E}-08$ & 3.07E-06 & $1.60 \mathrm{E}-05$ & $6.14 \mathrm{E}-06$ & 10 & $\begin{array}{c}\text { TPT1,WEE1,TPX2, } \\
\text { AURKA,FBXO5,B } \\
\text { UB1,ECT2,CCNB1, } \\
\text { KIF20A,CDK1 }\end{array}$ \\
\hline 138080 & $\begin{array}{l}\text { Aurora B } \\
\text { signaling }\end{array}$ & $3.01 \mathrm{E}-06$ & 8.03E-05 & $4.18 \mathrm{E}-04$ & 3.07E-04 & 8 & $\begin{array}{c}\text { CENPA, VIM,BIRC } \\
\text { 5,AURKA,NCAPG, } \\
\text { BUB1,KIF20A,AU } \\
\text { RKB }\end{array}$ \\
\hline 169351 & $\begin{array}{l}\text { Validated targets } \\
\text { of C-MYC }\end{array}$ & $3.15 \mathrm{E}-06$ & 8.03E-05 & $4.18 \mathrm{E}-04$ & $3.21 \mathrm{E}-04$ & 11 & $\begin{array}{l}\text { MMP9,HSPA4,HSP } \\
\text { D1,ID2,BIRC5,MY }\end{array}$ \\
\hline
\end{tabular}


medRxiv preprint doi: https://doi.org/10.1101/2020.12.21.20248616; this version posted December 23, 2020. The copyright holder for this preprint (which was not certified by peer review) is the author/funder, who has granted medRxiv a license to display the preprint in perpetuity. All rights reserved. No reuse allowed without permission.

\begin{tabular}{|c|c|c|c|c|c|c|c|}
\hline & $\begin{array}{l}\text { transcriptional } \\
\text { activation }\end{array}$ & & & & & & $\begin{array}{c}\text { C,GAPDH,PTMA,C } \\
\text { CNB1,CDCA7,EIF } \\
4 \mathrm{E}\end{array}$ \\
\hline 137987 & $\begin{array}{l}\text { Regulation of } \\
\text { Telomerase }\end{array}$ & $2.02 \mathrm{E}-02$ & $1.47 \mathrm{E}-01$ & 7.65E-01 & $1.00 \mathrm{E}+00$ & 5 & $\begin{array}{c}\text { HNRNPC,PTGES3, } \\
\text { MYC,SP3,SAP30 }\end{array}$ \\
\hline 138006 & $\begin{array}{c}\text { ATF-2 } \\
\text { transcription factor } \\
\text { network }\end{array}$ & 4.14E-02 & $2.01 \mathrm{E}-01$ & $1.00 \mathrm{E}+00$ & $1.00 \mathrm{E}+00$ & 4 & $\begin{array}{c}\text { PLAU,CSRP2,CXC } \\
\text { L8,CCNA2 }\end{array}$ \\
\hline 138035 & $\begin{array}{c}\text { Glypican } 2 \\
\text { network }\end{array}$ & $4.70 \mathrm{E}-02$ & $2.18 \mathrm{E}-01$ & $1.00 \mathrm{E}+00$ & $1.00 \mathrm{E}+00$ & 1 & MDK \\
\hline 137917 & $\begin{array}{c}\text { Angiopoietin } \\
\text { receptor Tie2- } \\
\text { mediated signaling }\end{array}$ & $1.06 \mathrm{E}-01$ & $3.47 \mathrm{E}-01$ & $1.00 \mathrm{E}+00$ & $1.00 \mathrm{E}+00$ & 3 & $\begin{array}{c}\text { ETS1,ANGPT2,ITG } \\
\text { A5 }\end{array}$ \\
\hline 137974 & $\begin{array}{l}\text { Caspase cascade } \\
\text { in apoptosis }\end{array}$ & $1.16 \mathrm{E}-01$ & 3.67E-01 & $1.00 \mathrm{E}+00$ & $1.00 \mathrm{E}+00$ & 3 & $\begin{array}{c}\text { VIM,CASP4,TFAP } \\
2 \mathrm{~A}\end{array}$ \\
\hline 137981 & $\begin{array}{l}\text { Insulin-mediated } \\
\text { glucose transport }\end{array}$ & $1.51 \mathrm{E}-01$ & $3.87 \mathrm{E}-01$ & $1.00 \mathrm{E}+00$ & $1.00 \mathrm{E}+00$ & 2 & ASIP,RHOQ \\
\hline
\end{tabular}

REACTOME

RPL23A,RPL29,RP L31,RPLP1,RPN2, RPS2,RPS3,RPS3A, RPS4X,RPS4Y1,RP S7,RPS8,RPS10,RP S11,RPS12,RPS13, RPS15A,RPS18,RP S25,RPS26,RPS27, RPS27A,RPS28,RP S29,RPL23,EIF3M, RPL14,EEF1A1,EE F1B2,SEC61G,EIF4 E,RPL6,RPL7,RPL 7A,RPL9,RPL15,R

PL18A

\begin{tabular}{|c|c|c|c|c|c|c|c|}
\hline 1270244 & $\begin{array}{l}\text { Extracellular } \\
\text { matrix } \\
\text { organization }\end{array}$ & $2.38 \mathrm{E}-09$ & 7.13E-08 & $5.23 \mathrm{E}-07$ & $2.07 \mathrm{E}-06$ & 27 & $\begin{array}{c}\text { A2M,MMP7,MMP9 } \\
\text {,PLOD2,VCAM1,C } \\
\text { OL1A1,COL1A2,C } \\
\text { OL3A1,COL4A1,C } \\
\text { OL5A1,COL5A2,C } \\
\text { OL6A3,COL8A1,T } \\
\text { NC,ITGA5,ADAM } \\
\text { TS9,CAST,LAMA4 } \\
\text {,LAMB1,LAMC1,S } \\
\text { ERPINH1,TIMP1,P } \\
\text { 4HA1,LOX,CD44,P } \\
\text { COLCE2,LUM }\end{array}$ \\
\hline 1269763 & $\begin{array}{c}\text { Cell Cycle, } \\
\text { Mitotic }\end{array}$ & $7.13 \mathrm{E}-08$ & $2.00 \mathrm{E}-06$ & $1.47 \mathrm{E}-05$ & $6.18 \mathrm{E}-05$ & 34 & $\begin{array}{c}\text { PTTG1,CENPA,CE } \\
\text { NPF,RPS27,HMMR } \\
\text {,RPS27A,CDC45,P } \\
\text { HLDA1,WEE1,BIR } \\
\text { C5,PPP2CB,TPX2, } \\
\text { GINS2,CDCA5,MY }\end{array}$ \\
\hline
\end{tabular}


medRxiv preprint doi: https://doi.org/10.1101/2020.12.21.20248616; this version posted December 23, 2020. The copyright holder for this preprint (which was not certified by peer review) is the author/funder, who has granted medRxiv a license to display the preprint in perpetuity. All rights reserved. No reuse allowed without permission.

1268677

1457780

Neutrophil

degranulation

7.12E-07

1.14E-04

$6.18 \mathrm{E}-04$

31 proteins

8.44E-08

2.15E-06

$1.58 \mathrm{E}-05$

7.32E-05

72

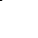

R

L31,HIF1A,RPLP1, RPN2,RPS2,RPS3,

RPS3A,RPS4X,RPS 4Y1,RPS7,RPS8,RP S10,RPS11,RPS12, RPS13,RPS15A,RP S18,RPS25,RPS26, RPS27,RPS27A,RP S28,RPS29,HNRNP C,HNRNPK,RPL23 ,SAA1,EIF3M,HSP D1,BIRC5,YY1,IG FBP2,IGFBP3,IGF BP4,CCT8,SUMO2, CXCL8,MYC,SP3, UAP1,BCHE,AUR KA,TUBB6,SEC24 D,LYPD1,ADAMT S9,GNG10,RAB13, KDELR2,TUBA1C, UBE2C,RPL14,TF AP2A,CCNA2,EEF 1A1,TGFBI,USP8,E EF1B2,SEC61G,EI F4E,LYZ,RPL6,TO P2A,RPL7,RPL7A, RPL9,PCNA,RPL1 5,AURKB,RPL18A

SERPINA3,HLAA,HEBP2,HLAH,HMGB1, CHI3L1, FABP5,S100A11,H P,BRI3,PLAU,MM P9,HSPA1A,ANXA 2,PRDX4,PPIA,CD 93,PRCP,FTH1,CC T8,GGH,BST2,PY

GL,RAP1B,LGALS 3,EEF1A1,RNASE2 ,CD44,CD68,TNFA IP6,LYZ 
medRxiv preprint doi: https://doi.org/10.1101/2020.12.21.20248616; this version posted December 23, 2020. The copyright holder for this

preprint (which was not certified by peer review) is the author/funder, who has granted medRxiv a license to display the preprint in perpetuity. All rights reserved. No reuse allowed without permission.

\begin{tabular}{|c|c|c|c|c|c|c|c|}
\hline & & & & & & & $\begin{array}{c}\text {,GNG10,KIF11,KIF } \\
\text { C1,RAP1B,CAV1,T } \\
\text { FPI,KIF20A,SLC16 } \\
\text { A3,TIMP1,CD44,C } \\
\text { DK2 }\end{array}$ \\
\hline 1269310 & $\begin{array}{c}\text { Cytokine } \\
\text { Signaling in } \\
\text { Immune system }\end{array}$ & $1.25 \mathrm{E}-03$ & $1.43 \mathrm{E}-02$ & $1.05 \mathrm{E}-01$ & $1.00 \mathrm{E}+00$ & 32 & $\begin{array}{c}\text { HIF1A,HLA- } \\
\text { A,HLA-G,HLA- } \\
\text { H,RPS27A,HMOX1 } \\
\text {,F13A1,TNFRSF12 } \\
\text { A,SAA1,IFI30,CCL } \\
\text { 20,MMP9,VCAM1, } \\
\text { COL1A2,VEGFA,V } \\
\text { IM,ANXA1,BIRC5, } \\
\text { PPP2CB,IL1RAP,C } \\
\text { XCL8,MYC,IL13R } \\
\text { A2,GBP1,GBP2,SO } \\
\text { CS2,BST2,KPNA2, } \\
\text { RAP1B,TIMP1,EIF } \\
\text { 4E,CD44 }\end{array}$ \\
\hline 1270414 & $\begin{array}{c}\text { Cellular responses } \\
\text { to stress }\end{array}$ & $2.37 \mathrm{E}-02$ & $1.54 \mathrm{E}-01$ & $1.00 \mathrm{E}+00$ & $1.00 \mathrm{E}+00$ & 18 & $\begin{array}{c}\text { HIF1A,ETS1,RPS2 } \\
\text { 7A,HSPA1A,HSPA } \\
\text { 4,VEGFA,GPX8,N } \\
\text { OX4,PTGES3,SOD } \\
\text { 2,CXCL8,CA9,UBE } \\
\text { 2C,GPX7,E2F2,CC } \\
\text { NA2,EEF1A1,CDK } \\
2\end{array}$ \\
\hline 1269203 & $\begin{array}{l}\text { Innate Immune } \\
\text { System }\end{array}$ & $8.38 \mathrm{E}-02$ & $3.28 \mathrm{E}-01$ & $1.00 \mathrm{E}+00$ & $1.00 \mathrm{E}+00$ & 39 & $\begin{array}{c}\text { SERPINA3,HLA- } \\
\text { A,HEBP2,HLA- } \\
\text { H,HMGB1,RPS27A } \\
\text {,LY96,CHI3L1,FA } \\
\text { BP5,S100A11,SAA } \\
\text { 1,HP,BRI3,PLAU, } \\
\text { MMP9,HSPA1A,A } \\
\text { NXA2,PRDX4,CFI, } \\
\text { PPIA,CD93,PPP2C } \\
\text { B,PRCP,FTH1,CCT } \\
\text { 8,GGH,BST2,PYG } \\
\text { L,C1S,RAP1B,CAS } \\
\text { P4,ACTR3,LGALS } \\
\text { 3,EEF1A1,RNASE2 } \\
\text {,CD44,CD68,TNFA } \\
\text { IP6,LYZ }\end{array}$ \\
\hline 1269688 & $\begin{array}{c}\text { Processing of } \\
\text { Capped Intron- } \\
\text { Containing Pre- } \\
\text { mRNA }\end{array}$ & $1.38 \mathrm{E}-01$ & $4.25 \mathrm{E}-01$ & $1.00 \mathrm{E}+00$ & $1.00 \mathrm{E}+00$ & 9 & $\begin{array}{c}\text { HNRNPA1,LSM5, } \\
\text { HNRNPC,HNRNP } \\
\text { K,WTAP,SF3B6,S } \\
\text { NRPG,SLBP,EIF4E }\end{array}$ \\
\hline
\end{tabular}

Gen MAPP

$\begin{array}{cc}\text { MAP00500 } & \begin{array}{c}\text { Starch and sucrose } \\ \text { metabolism }\end{array}\end{array}$

1.47E-02 2.35E-01

7.93E-01

Nitrogen metabolism
8.80E-02

5.12E-01

$1.00 \mathrm{E}+00$

$1.00 \mathrm{E}+00$ 
medRxiv preprint doi: https://doi.org/10.1101/2020.12.21.20248616; this version posted December 23, 2020. The copyright holder for this preprint (which was not certified by peer review) is the author/funder, who has granted medRxiv a license to display the preprint in perpetuity.

All rights reserved. No reuse allowed without permission.

\begin{tabular}{|c|c|c|c|c|c|c|c|}
\hline MAP00630 & $\begin{array}{l}\text { Glyoxylate and } \\
\text { dicarboxylate } \\
\text { metabolism }\end{array}$ & $2.14 \mathrm{E}-01$ & $5.12 \mathrm{E}-01$ & $1.00 \mathrm{E}+00$ & $1.00 \mathrm{E}+00$ & 1 & MTHFD2 \\
\hline MAP00330 & $\begin{array}{l}\text { Arginine and } \\
\text { proline } \\
\text { metabolism }\end{array}$ & $2.28 \mathrm{E}-01$ & $5.12 \mathrm{E}-01$ & $1.00 \mathrm{E}+00$ & $1.00 \mathrm{E}+00$ & 2 & SMS,P4HA1 \\
\hline MAP00670 & $\begin{array}{l}\text { One carbon pool } \\
\text { by folate }\end{array}$ & $2.33 \mathrm{E}-01$ & $5.12 \mathrm{E}-01$ & $1.00 \mathrm{E}+00$ & $1.00 \mathrm{E}+00$ & 1 & MTHFD2 \\
\hline MAP00790 & $\begin{array}{c}\text { Folate } \\
\text { biosynthesis }\end{array}$ & $2.51 \mathrm{E}-01$ & $5.12 \mathrm{E}-01$ & $1.00 \mathrm{E}+00$ & $1.00 \mathrm{E}+00$ & 1 & GGH \\
\hline MAP00561 & $\begin{array}{l}\text { Glycerolipid } \\
\text { metabolism }\end{array}$ & $3.08 \mathrm{E}-01$ & $5.12 \mathrm{E}-01$ & $1.00 \mathrm{E}+00$ & $1.00 \mathrm{E}+00$ & 2 & LPL,TPI1 \\
\hline MAP00860 & $\begin{array}{l}\text { Porphyrin and } \\
\text { chlorophyll } \\
\text { metabolism }\end{array}$ & $3.52 \mathrm{E}-01$ & $5.12 \mathrm{E}-01$ & $1.00 \mathrm{E}+00$ & $1.00 \mathrm{E}+00$ & 1 & HMOX1 \\
\hline MAP00051 & $\begin{array}{l}\text { Fructose and } \\
\text { mannose } \\
\text { metabolism }\end{array}$ & 4.39E-01 & $5.85 \mathrm{E}-01$ & $1.00 \mathrm{E}+00$ & $1.00 \mathrm{E}+00$ & 1 & TPI1 \\
\hline MAP00361 & $\begin{array}{c}\text { gamma } \\
\text { Hexachlorocycloh } \\
\text { exane degradation }\end{array}$ & $5.03 \mathrm{E}-01$ & $6.19 \mathrm{E}-01$ & $1.00 \mathrm{E}+00$ & $1.00 \mathrm{E}+00$ & 1 & CYP51A1 \\
\hline \multicolumn{8}{|c|}{ MSigDB C2 BIOCARTA (v6.0) } \\
\hline M5884 & $\begin{array}{l}\text { Ensemble of genes } \\
\text { encoding core } \\
\text { extracellular } \\
\text { matrix including } \\
\text { ECM } \\
\text { glycoproteins, } \\
\text { collagens and } \\
\text { proteoglycans }\end{array}$ & $7.93 \mathrm{E}-11$ & $7.48 \mathrm{E}-09$ & $4.01 \mathrm{E}-08$ & $9.44 \mathrm{E}-09$ & 28 & $\begin{array}{c}\text { EMILIN2,MGP,AE } \\
\text { BP1,CTHRC1,SRP } \\
\text { X,COL1A1,COL1A } \\
\text { 2,COL3A1,COL4A } \\
\text { 1,COL5A1,COL5A } \\
\text { 2,COL6A3,COL8A } \\
\text { 1,MXRA5,TNC,PO } \\
\text { STN,IGFBP2,IGFB } \\
\text { P3,IGFBP4,SRPX2, } \\
\text { LAMA4,LAMB1,E } \\
\text { SM1,LAMC1,TGF } \\
\text { BI,PCOLCE2,TNF } \\
\text { AIP6,LUM }\end{array}$ \\
\hline M5889 & $\begin{array}{l}\text { Ensemble of genes } \\
\text { encoding } \\
\text { extracellular } \\
\text { matrix and } \\
\text { extracellular } \\
\text { matrix-associated } \\
\text { proteins }\end{array}$ & $1.26 \mathrm{E}-10$ & 7.48E-09 & 4.01E-08 & $1.50 \mathrm{E}-08$ & 59 & $\begin{array}{c}\text { A2M,SERPINA3,E } \\
\text { MILIN2,MDK,F13 } \\
\text { A1,S100A3,S100A4 } \\
\text {,S100A6,S100A10, } \\
\text { S100A11,MGP,AE } \\
\text { BP1,CTHRC1,PLA } \\
\text { U,C1QTNF1,SRPX, } \\
\text { MMP7,CCL20,MM } \\
\text { P9,PLOD2,COL1A } \\
\text { 1,COL1A2,VEGFA, } \\
\text { COL3A1,COL4A1, } \\
\text { COL5A1,COL5A2, } \\
\text { COL6A3,COL8A1, } \\
\text { MXRA5,ANGPT2, } \\
\text { TNC,ANXA1,ANX } \\
\text { A2,GDF15,POSTN, }\end{array}$ \\
\hline
\end{tabular}


medRxiv preprint doi: https://doi.org/10.1101/2020.12.21.20248616; this version posted December 23, 2020. The copyright holder for this preprint (which was not certified by peer review) is the author/funder, who has granted medRxiv a license to display the preprint in perpetuity. All rights reserved. No reuse allowed without permission.

\begin{tabular}{|c|c|c|c|c|c|c|c|}
\hline M17370 & $\begin{array}{l}\text { Role of Ran in } \\
\text { mitotic spindle } \\
\text { regulation }\end{array}$ & $5.87 \mathrm{E}-05$ & $1.40 \mathrm{E}-03$ & 7.49E-03 & $6.99 \mathrm{E}-03$ & 4 & $\begin{array}{c}\text { TPX2,AURKA,KP } \\
\text { NA2,RAN }\end{array}$ \\
\hline M5885 & $\begin{array}{l}\text { Ensemble of genes } \\
\text { encoding ECM- } \\
\text { associated proteins } \\
\text { including ECM- } \\
\text { affilaited proteins, } \\
\text { ECM regulators } \\
\text { and secreted } \\
\text { factors }\end{array}$ & $1.99 \mathrm{E}-03$ & $3.16 \mathrm{E}-02$ & $1.70 \mathrm{E}-01$ & $2.37 \mathrm{E}-01$ & 31 & $\begin{array}{c}\text { A2M,SERPINA3,M } \\
\text { DK,F13A1,S100A3, } \\
\text { S100A4,S100A6,S1 } \\
\text { 00A10,S100A11,PL } \\
\text { AU,C1QTNF1,MM } \\
\text { P7,CCL20,MMP9,P } \\
\text { LOD2,VEGFA,AN } \\
\text { GPT2,ANXA1,AN } \\
\text { XA2,GDF15,CLEC } \\
\text { 2D,CXCL8,ADAM } \\
\text { TS9,SERPINH1,LG } \\
\text { ALS1,LGALS3,FS } \\
\text { TL1,TIMP1,TIMP4, } \\
\text { P4HA1,LOX }\end{array}$ \\
\hline M3468 & $\begin{array}{l}\text { Genes encoding } \\
\text { enzymes and their } \\
\text { regulators } \\
\text { involved in the } \\
\text { remodeling of the } \\
\text { extracellular } \\
\text { matrix }\end{array}$ & $4.58 \mathrm{E}-03$ & 4.19E-02 & $2.25 \mathrm{E}-01$ & $5.45 \mathrm{E}-01$ & 13 & $\begin{array}{c}\text { A2M,SERPINA3,F } \\
\text { 13A1,PLAU,MMP7 } \\
\text {,MMP9,PLOD2,AD } \\
\text { AMTS9,SERPINH1 } \\
\text {,TIMP1,TIMP4,P4H } \\
\text { A1,LOX }\end{array}$ \\
\hline M5202 & $\begin{array}{l}\text { Hypoxia and p53 } \\
\text { in the } \\
\text { Cardiovascular } \\
\text { system }\end{array}$ & $1.66 \mathrm{E}-02$ & $1.16 \mathrm{E}-01$ & $6.22 \mathrm{E}-01$ & $1.00 \mathrm{E}+00$ & 3 & $\begin{array}{l}\text { HIF1A,HSPA1A,IG } \\
\text { FBP3 }\end{array}$ \\
\hline M16801 & $\begin{array}{l}\text { Genes related to } \\
\text { regulation of the } \\
\text { actin cytoskeleton }\end{array}$ & $4.98 \mathrm{E}-02$ & $1.97 \mathrm{E}-01$ & $1.00 \mathrm{E}+00$ & $1.00 \mathrm{E}+00$ & 3 & $\begin{array}{c}\text { RPS4X,ACTG2,AC } \\
\text { TR3 }\end{array}$ \\
\hline M5882 & $\begin{array}{l}\text { Genes encoding } \\
\text { proteoglycans }\end{array}$ & $2.02 \mathrm{E}-01$ & $5.29 \mathrm{E}-01$ & $1.00 \mathrm{E}+00$ & $1.00 \mathrm{E}+00$ & 2 & ESM1,LUM \\
\hline
\end{tabular}


medRxiv preprint doi: https://doi.org/10.1101/2020.12.21.20248616; this version posted December 23, 2020. The copyright holder for this preprint (which was not certified by peer review) is the author/funder, who has granted medRxiv a license to display the preprint in perpetuity. All rights reserved. No reuse allowed without permission.

\begin{tabular}{|c|c|c|c|c|c|c|c|}
\hline M5880 & $\begin{array}{l}\text { Genes encoding } \\
\text { proteins affiliated } \\
\text { structurally or } \\
\text { functionally to } \\
\text { extracellular } \\
\text { matrix proteins }\end{array}$ & $2.22 \mathrm{E}-01$ & $5.29 \mathrm{E}-01$ & $1.00 \mathrm{E}+00$ & $1.00 \mathrm{E}+00$ & 6 & $\begin{array}{c}\text { C1QTNF1,ANXA1, } \\
\text { ANXA2,CLEC2D,L } \\
\text { GALS1,LGALS3 }\end{array}$ \\
\hline
\end{tabular}

Panther DB

\begin{tabular}{|c|c|c|c|c|c|c|c|}
\hline P00034 & $\begin{array}{c}\text { Integrin signalling } \\
\text { pathway }\end{array}$ & $4.47 \mathrm{E}-05$ & $2.59 \mathrm{E}-03$ & $1.20 \mathrm{E}-02$ & $2.59 \mathrm{E}-03$ & 14 & $\begin{array}{c}\text { COL1A1,COL1A2, } \\
\text { COL3A1,COL4A1, } \\
\text { COL5A1,COL5A2, } \\
\text { COL6A3,COL8A1,I } \\
\text { TGA5,RAP1B,LA } \\
\text { MA4,LAMB1,LAM } \\
\text { C1,CAV1 }\end{array}$ \\
\hline P00059 & p53 pathway & $2.69 \mathrm{E}-03$ & $7.80 \mathrm{E}-02$ & $3.62 \mathrm{E}-01$ & $1.56 \mathrm{E}-01$ & 7 & $\begin{array}{c}\text { HMGB1,PPP2CB,I } \\
\text { GFBP3,SUMO2,CC } \\
\text { NB1,CDK1,CDK2 }\end{array}$ \\
\hline P00011 & Blood coagulation & $1.46 \mathrm{E}-02$ & $2.82 \mathrm{E}-01$ & $1.00 \mathrm{E}+00$ & $8.47 \mathrm{E}-01$ & 4 & F2R,F3,PLAU,TFPI \\
\hline P04398 & $\begin{array}{c}\text { p53 pathway } \\
\text { feedback loops } 2\end{array}$ & $2.17 \mathrm{E}-02$ & $3.15 \mathrm{E}-01$ & $1.00 \mathrm{E}+00$ & $1.00 \mathrm{E}+00$ & 4 & $\begin{array}{c}\text { PPP2CB,MYC,CC } \\
\text { NA2,CDK2 }\end{array}$ \\
\hline P00004 & $\begin{array}{c}\text { Alzheimer } \\
\text { disease-presenilin } \\
\text { pathway }\end{array}$ & $2.82 \mathrm{E}-01$ & $8.83 \mathrm{E}-01$ & $1.00 \mathrm{E}+00$ & $1.00 \mathrm{E}+00$ & 4 & $\begin{array}{l}\text { ACTG2,MMP7,M } \\
\text { MP9,CD44 }\end{array}$ \\
\hline P00005 & Angiogenesis & $2.86 \mathrm{E}-01$ & $8.83 \mathrm{E}-01$ & $1.00 \mathrm{E}+00$ & $1.00 \mathrm{E}+00$ & 5 & $\begin{array}{c}\text { HIF1 A,ETS1,F3,VE } \\
\text { GFA,ANGPT2 }\end{array}$ \\
\hline P00031 & $\begin{array}{c}\text { Inflammation } \\
\text { mediated by } \\
\text { chemokine and } \\
\text { cytokine signaling } \\
\text { pathway }\end{array}$ & 3.03E-01 & 8.83E-01 & $1.00 \mathrm{E}+00$ & $1.00 \mathrm{E}+00$ & 6 & $\begin{array}{c}\text { ACTG2,CCL20,CO } \\
\text { L6A3,FPR3,CXCL8 } \\
\text {,GNG10 }\end{array}$ \\
\hline P00029 & $\begin{array}{l}\text { Huntington } \\
\text { disease }\end{array}$ & $3.36 \mathrm{E}-01$ & $8.83 \mathrm{E}-01$ & $1.00 \mathrm{E}+00$ & $1.00 \mathrm{E}+00$ & 4 & $\begin{array}{c}\text { ACTG2,GAPDH,T } \\
\text { UBB6,RHOQ }\end{array}$ \\
\hline P00042 & $\begin{array}{c}\text { Muscarinic } \\
\text { acetylcholine } \\
\text { receptor } 1 \text { and } 3 \\
\text { signaling pathway }\end{array}$ & $3.77 \mathrm{E}-01$ & $8.83 \mathrm{E}-01$ & $1.00 \mathrm{E}+00$ & $1.00 \mathrm{E}+00$ & 2 & BCHE,GNG10 \\
\hline P00035 & $\begin{array}{l}\text { Interferon-gamma } \\
\text { signaling pathway }\end{array}$ & 4.91E-01 & $8.83 \mathrm{E}-01$ & $1.00 \mathrm{E}+00$ & $1.00 \mathrm{E}+00$ & 1 & SOCS2 \\
\hline
\end{tabular}

Pathway Ontology

\begin{tabular}{|c|c|c|c|c|c|c|c|}
\hline PW:0000021 & hypertension & $5.23 \mathrm{E}-05$ & $2.67 \mathrm{E}-03$ & $1.21 \mathrm{E}-02$ & $2.67 \mathrm{E}-03$ & 3 & $\begin{array}{c}\text { HMOX1,SOD2,LO } \\
\mathrm{X}\end{array}$ \\
\hline PW:0000385 & $\begin{array}{l}\text { G2/M DNA } \\
\text { replication } \\
\text { checkpoint }\end{array}$ & $5.77 \mathrm{E}-04$ & $7.36 \mathrm{E}-03$ & $3.33 \mathrm{E}-02$ & $2.95 \mathrm{E}-02$ & 2 & CCNB1,CDK1 \\
\hline PW:0000238 & insulin-like growth & $2.50 \mathrm{E}-03$ & $2.12 \mathrm{E}-02$ & $9.59 \mathrm{E}-02$ & $1.27 \mathrm{E}-01$ & 1 & IGFBP2,IGFBP3,IG \\
\hline
\end{tabular}


medRxiv preprint doi: https://doi.org/10.1101/2020.12.21.20248616; this version posted December 23, 2020. The copyright holder for this preprint (which was not certified by peer review) is the author/funder, who has granted medRxiv a license to display the preprint in perpetuity. All rights reserved. No reuse allowed without permission.

factor signaling

FBP4

PW:0000482

$$
\text { lipoprotein }
$$

3.70E-02

$1.45 \mathrm{E}-01$

$6.54 \mathrm{E}-01$

$1.00 \mathrm{E}+00$

2

APOC1,LPL

PW:0000189

$$
\begin{gathered}
\text { folate mediated } \\
\text { one-carbon } \\
\text { metabolic }
\end{gathered}
$$

4.83E-02

$1.45 \mathrm{E}-01$

$6.54 \mathrm{E}-01$

$1.00 \mathrm{E}+00$

2

MTHFD2,GGH

PW:0000183

the proteolytic

6.97E-02

1.69E-01

7.64E-0

$1.00 \mathrm{E}+00$

1

CAST involving calcium-

dependent

proteases

PW:0000102

$$
\begin{gathered}
\text { The extracellular } \\
\text { signal-regulated } \\
\text { RAF/MEK/ERK } \\
\text { signaling }
\end{gathered}
$$

PW:0000559

hexosamine

biosynthetic

$1.26 \mathrm{E}-01$

2.30E-01

$1.00 \mathrm{E}+00$

$1.00 \mathrm{E}+00$

2

MDK,F2R

PW:0000398

homocysteine

3.52E-01

4.60E-01

\begin{tabular}{|c|c|c|c|c|c|c|c|}
\hline SMP00058 & $\begin{array}{l}\text { Starch and } \\
\text { Sucrose } \\
\text { Metabolism }\end{array}$ & $9.72 \mathrm{E}-03$ & $1.42 \mathrm{E}-01$ & $6.42 \mathrm{E}-01$ & $4.96 \mathrm{E}-01$ & 3 & $\begin{array}{c}\text { AMY1A,GBE1,PY } \\
\text { GL }\end{array}$ \\
\hline SMP00048 & $\begin{array}{l}\text { Nicotinate and } \\
\text { Nicotinamide } \\
\text { Metabolism }\end{array}$ & $3.18 \mathrm{E}-02$ & $1.42 \mathrm{E}-01$ & $6.42 \mathrm{E}-01$ & $1.00 \mathrm{E}+00$ & 2 & NNMT,NAMPT \\
\hline SMP00040 & Glycolysis & $3.70 \mathrm{E}-02$ & $1.42 \mathrm{E}-01$ & $6.42 \mathrm{E}-01$ & $1.00 \mathrm{E}+00$ & 2 & GAPDH,TPI1 \\
\hline SMP00287 & $\begin{array}{c}\text { Tranexamic Acid } \\
\text { Pathway }\end{array}$ & $5.43 \mathrm{E}-02$ & $1.42 \mathrm{E}-01$ & $6.42 \mathrm{E}-01$ & $1.00 \mathrm{E}+00$ & 2 & F3,F13A1 \\
\hline SMP00383 & $\begin{array}{l}\text { Obesity / } \\
\text { Metabolic } \\
\text { Syndrome }\end{array}$ & $1.95 \mathrm{E}-01$ & $3.43 \mathrm{E}-01$ & $1.00 \mathrm{E}+00$ & $1.00 \mathrm{E}+00$ & 1 & LPL \\
\hline SMP00005 & $\begin{array}{c}\text { Folate and Pterine } \\
\text { Biosynthesis }\end{array}$ & $1.95 \mathrm{E}-01$ & $3.43 \mathrm{E}-01$ & $1.00 \mathrm{E}+00$ & $1.00 \mathrm{E}+00$ & 1 & GGH \\
\hline SMP00029 & $\begin{array}{l}\text { Selenoamino Acid } \\
\text { Metabolism }\end{array}$ & $1.95 \mathrm{E}-01$ & $3.43 \mathrm{E}-01$ & $1.00 \mathrm{E}+00$ & $1.00 \mathrm{E}+00$ & 1 & PAPSS1 \\
\hline SMP00264 & $\begin{array}{c}\text { Dipyridamole } \\
\text { Pathway }\end{array}$ & $2.51 \mathrm{E}-01$ & $3.55 \mathrm{E}-01$ & $1.00 \mathrm{E}+00$ & $1.00 \mathrm{E}+00$ & 1 & F2R \\
\hline SMP00045 & $\begin{array}{c}\text { Amino Sugar } \\
\text { Metabolism }\end{array}$ & $2.86 \mathrm{E}-01$ & $3.94 \mathrm{E}-01$ & $1.00 \mathrm{E}+00$ & $1.00 \mathrm{E}+00$ & 1 & UAP1 \\
\hline SMP00006 & $\begin{array}{c}\text { Tyrosine } \\
\text { Metabolism }\end{array}$ & $4.39 \mathrm{E}-01$ & $4.57 \mathrm{E}-01$ & $1.00 \mathrm{E}+00$ & $1.00 \mathrm{E}+00$ & 1 & NOX4 \\
\hline
\end{tabular}

$1.00 \mathrm{E}+$

3.52E-01

4.60E-01

$1.00 \mathrm{E}+00$

biosynthetic

\section{SMPDB}


medRxiv preprint doi: https://doi.org/10.1101/2020.12.21.20248616; this version posted December 23, 2020. The copyright holder for this preprint (which was not certified by peer review) is the author/funder, who has granted medRxiv a license to display the preprint in perpetuity. All rights reserved. No reuse allowed without permission.

Table 3 The enriched pathway terms of the down regulated differentially expressed genes

BIOCYC

\begin{tabular}{|c|c|c|c|c|c|c|c|}
\hline Pathway ID & Pathway Name & P-value & $\begin{array}{l}\text { FDR } \\
\text { B\&H }\end{array}$ & $\begin{array}{l}\text { FDR } \\
\text { B\&Y }\end{array}$ & Bonferroni & $\begin{array}{c}\text { Gene } \\
\text { Count }\end{array}$ & Gene \\
\hline 1108774 & homocarnosine biosynthesis & $2.04 \mathrm{E}-02$ & $2.49 \mathrm{E}-01$ & $9.78 \mathrm{E}-01$ & $5.72 \mathrm{E}-01$ & 1 & CARNS1 \\
\hline 703095 & fatty acid alpha-oxidation III & $3.96 \mathrm{E}-02$ & $2.49 \mathrm{E}-01$ & $9.78 \mathrm{E}-01$ & $1.00 \mathrm{E}+00$ & 1 & $\mathrm{FA} 2 \mathrm{H}$ \\
\hline 547502 & $\begin{array}{c}\text { glutamine } \\
\text { degradation/glutamate } \\
\text { biosynthesis }\end{array}$ & $3.96 \mathrm{E}-02$ & $2.49 \mathrm{E}-01$ & $9.78 \mathrm{E}-01$ & $1.00 \mathrm{E}+00$ & 1 & GLS \\
\hline 545293 & retinol biosynthesis & 4.45E-02 & $2.49 \mathrm{E}-01$ & $9.78 \mathrm{E}-01$ & $1.00 \mathrm{E}+00$ & 2 & CES4A,DHRS9 \\
\hline 545319 & creatine-phosphate biosynthesis & $5.88 \mathrm{E}-02$ & $2.75 \mathrm{E}-01$ & $1.00 \mathrm{E}+00$ & $1.00 \mathrm{E}+00$ & 1 & CKMT1B \\
\hline 1108775 & $\begin{array}{l}\text { lysine degradation II } \\
\text { (pipecolate pathway) }\end{array}$ & $7.76 \mathrm{E}-02$ & $3.11 \mathrm{E}-01$ & $1.00 \mathrm{E}+00$ & $1.00 \mathrm{E}+00$ & 1 & CRYM \\
\hline 142436 & bupropion degradation & $9.61 \mathrm{E}-02$ & $3.36 \mathrm{E}-01$ & $1.00 \mathrm{E}+00$ & $1.00 \mathrm{E}+00$ & 1 & CYP4X1 \\
\hline 545299 & $\mathrm{C} 20$ prostanoid biosynthesis & $1.32 \mathrm{E}-01$ & 3.69E-01 & $1.00 \mathrm{E}+00$ & $1.00 \mathrm{E}+00$ & 1 & PTGDS \\
\hline 782395 & $\begin{array}{l}\text { purine deoxyribonucleosides } \\
\text { salvage }\end{array}$ & $2.15 \mathrm{E}-01$ & $4.02 \mathrm{E}-01$ & $1.00 \mathrm{E}+00$ & $1.00 \mathrm{E}+00$ & 1 & AK5 \\
\hline 545328 & $\begin{array}{l}\text { D-myo-inositol }(1,3,4)- \\
\text { trisphosphate biosynthesis }\end{array}$ & $2.62 \mathrm{E}-01$ & 4.07E-01 & $1.00 \mathrm{E}+00$ & $1.00 \mathrm{E}+00$ & 1 & ITPKA \\
\hline
\end{tabular}

\section{KEGG}

\begin{tabular}{|c|c|c|c|c|c|c|c|}
\hline 377263 & GABAergic synapse & $6.88 \mathrm{E}-15$ & $1.29 \mathrm{E}-12$ & $7.52 \mathrm{E}-12$ & $1.29 \mathrm{E}-12$ & 19 & $\begin{array}{c}\text { ADCY1,ADCY2,S } \\
\text { LC12A5,GABBR2, } \\
\text { SLC6A1,SLC32A1, } \\
\text { PRKCB,GABBR1, } \\
\text { GABRA2,GABRA5 } \\
\text {,GABRB1,GABRB } \\
\text { 3,GABRG2,GAD1, } \\
\text { GAD2,KCNJ6,GLS, } \\
\text { GNAO1,GNG3 }\end{array}$ \\
\hline 777534 & Insulin secretion & $1.22 \mathrm{E}-09$ & 7.66E-08 & 4.46E-07 & $2.30 \mathrm{E}-07$ & 14 & $\begin{array}{c}\text { ADCY1,CHRM3,A } \\
\text { DCY2,PRKCB,SN } \\
\text { AP25,ATP1B1,RIM } \\
\text { S2,STX1A,ABCC8, } \\
\text { KCNMA1,CAMK2 } \\
\text { A,CAMK2B,PCLO, } \\
\text { RAPGEF4 }\end{array}$ \\
\hline 373900 & Synaptic vesicle cycle & 3.40E-09 & $1.60 \mathrm{E}-07$ & $9.29 \mathrm{E}-07$ & $6.39 \mathrm{E}-07$ & 12 & $\begin{array}{l}\text { STX1B,SLC32A1, } \\
\text { DNM3,SNAP25,AT } \\
\text { P6V1G2,CPLX1,ST } \\
\text { X1A,STXBP1,SLC } \\
\text { 17A7,SYT1,DNM1, } \\
\text { UNC13C }\end{array}$ \\
\hline
\end{tabular}


medRxiv preprint doi: https://doi.org/10.1101/2020.12.21.20248616; this version posted December 23, 2020. The copyright holder for this preprint (which was not certified by peer review) is the author/funder, who has granted medRxiv a license to display the preprint in perpetuity. All rights reserved. No reuse allowed without permission.

213818

\begin{abstract}
Glutamatergic synapse
\end{abstract}
6.01E-08

2.21E-06

$1.29 \mathrm{E}-05$

1.13E-05

$1.38 \mathrm{E}-07$

$3.24 \mathrm{E}-0$

$1.88 \mathrm{E}-05$

$2.59 \mathrm{E}-05$

Endocytosis

7.49E-04

6.13E-03

3.56E-02

$1.41 \mathrm{E}-01$

7.07E-02

2.55E-01

$1.00 \mathrm{E}+00$

$1.00 \mathrm{E}+00$

83067

83098

132956
MAPK signaling pathway

Focal adhesion

Parkinson's disease

Metabolic pathways

$\begin{array}{llll}7.81 \mathrm{E}-01 & 9.53 \mathrm{E}-01 & 1.00 \mathrm{E}+00 & 1.00 \mathrm{E}+00 \\ 8.53 \mathrm{E}-01 & 9.66 \mathrm{E}-01 & 1.00 \mathrm{E}+00 & 1.00 \mathrm{E}+00\end{array}$

14

CY1,ADCY2,S

LC1 A2,SHANK3,S

HANK2,PRKCB,G

LS,SLC17A7,DLG4

,GNAO1,GNG3,GR

IN1,GRIN2C,GRM

3

17

PDE1A,ERBB3,AD

CY1,CHRM3,ADC

Y2,HTR2A,SLC8A

2,PRKCB,ATP2B2,

ITPKA,CACNA1I,

CACNA1E,CAMK

2A,CAMK2B,GRI

N1,GRIN2C,CCKB

$\mathrm{R}$

14 ERBB3,FGFR3,HS

PA2,AGAP1,AGAP

3,AMPH,SH3GL2,S

H3GL3,ARRB1,DN

M3,PRKCZ,RAB11

FIP4,KIF5C,DNM1

CACNG3,FGF12,F GFR3,HSPA2,ARR B1,PRKCB,MAPK

8IP3,CACNA1I,CA

CNA1E

PRKCB,RELN,PA

K6

SNCA,GPR37

CDS1,CKMT1B,U

GT8,ETNPPL,PLC

H2,GALNT9, ASPA

,OGDHL,PRODH,

GAD1,GAD2,NMN

AT2,ATP6V1G2,P

TGDS,ITPKA,AK5,

GLS,CKMT1A,CN

DP1,MTMR7,DHR

S9

\section{Pathway Interaction Database}

$\begin{array}{cccccccc}138028 & \text { Effects of Botulinumtoxin } & 1.82 \mathrm{E}-05 & 1.33 \mathrm{E}-03 & 6.47 \mathrm{E}-03 & 1.33 \mathrm{E}-03 & 4 & \begin{array}{c}\text { SNAP25,STX1A,S } \\ \text { TXBP1,SYT1 }\end{array} \\ 138013 & \text { Internalization of ErbB1 } & 4.52 \mathrm{E}-03 & 1.65 \mathrm{E}-01 & 8.04 \mathrm{E}-01 & 3.30 \mathrm{E}-01 & 4 & \begin{array}{c}\text { AMPH,SH3GL2,A } \\ \text { RHGEF7,DNM1 }\end{array} \\ 137982 & \text { S1P5 pathway } & 1.03 \mathrm{E}-02 & 2.51 \mathrm{E}-01 & 1.00 \mathrm{E}+00 & 7.52 \mathrm{E}-01 & 2 & \text { S1PR5,GNAO1 } \\ 137990 & \text { Regulation of CDC42 activity } & 1.20 \mathrm{E}-01 & 5.67 \mathrm{E}-01 & 1.00 \mathrm{E}+00 & 1.00 \mathrm{E}+00 & 2 & \text { ARHGEF7,DOCK9 } \\ 138019 & \text { p75(NTR)-mediated signaling } & 1.23 \mathrm{E}-01 & 5.67 \mathrm{E}-01 & 1.00 \mathrm{E}+00 & 1.00 \mathrm{E}+00 & 3 & \text { MAG,PRKCZ,OM }\end{array}$


medRxiv preprint doi: https://doi.org/10.1101/2020.12.21.20248616; this version posted December 23, 2020. The copyright holder for this preprint (which was not certified by peer review) is the author/funder, who has granted medRxiv a license to display the preprint in perpetuity. All rights reserved. No reuse allowed without permission.

G

\begin{tabular}{|c|c|c|c|c|c|c|c|}
\hline 169353 & $\begin{array}{l}\text { Validated targets of C-MYC } \\
\text { transcriptional repression }\end{array}$ & $1.23 \mathrm{E}-01$ & 5.67E-01 & $1.00 \mathrm{E}+00$ & $1.00 \mathrm{E}+00$ & 3 & $\begin{array}{c}\text { NDRG2,TMEFF2,S } \\
\text { FRP1 }\end{array}$ \\
\hline 138019 & p75(NTR)-mediated signaling & $1.23 \mathrm{E}-01$ & $5.67 \mathrm{E}-01$ & $1.00 \mathrm{E}+00$ & $1.00 \mathrm{E}+00$ & 3 & $\begin{array}{c}\text { MAG,PRKCZ,OM } \\
\text { G }\end{array}$ \\
\hline 137967 & $\begin{array}{l}\text { Trk receptor signaling mediated } \\
\text { by PI } 3 \mathrm{~K} \text { and PLC-gamma }\end{array}$ & $1.34 \mathrm{E}-01$ & $5.67 \mathrm{E}-01$ & $1.00 \mathrm{E}+00$ & $1.00 \mathrm{E}+00$ & 2 & $\begin{array}{c}\text { CAMK2A,EPB41L } \\
1\end{array}$ \\
\hline 137915 & $\begin{array}{l}\text { Signaling events regulated by } \\
\text { Ret tyrosine kinase }\end{array}$ & $1.76 \mathrm{E}-01$ & $6.02 \mathrm{E}-01$ & $1.00 \mathrm{E}+00$ & $1.00 \mathrm{E}+00$ & 2 & DOK6,SHANK3 \\
\hline 138014 & $\begin{array}{l}\text { Glucocorticoid receptor } \\
\text { regulatory network }\end{array}$ & 4.91E-01 & $7.54 \mathrm{E}-01$ & $1.00 \mathrm{E}+00$ & $1.00 \mathrm{E}+00$ & 2 & VIPR1,CDK5R1 \\
\hline
\end{tabular}

REACTOME

\begin{tabular}{|c|c|c|c|c|c|c|c|}
\hline 1268763 & Neuronal System & $7.37 \mathrm{E}-31$ & $3.58 \mathrm{E}-28$ & $2.42 \mathrm{E}-27$ & $3.58 \mathrm{E}-28$ & 52 & $\begin{array}{c}\text { DLGAP2,TSPOAP1 } \\
\text {,ADCY1,ADCY2,C } \\
\text { ACNG3,PPFIA2,G } \\
\text { ABBR2,SLC1A2,S } \\
\text { HANK3,SLC6A1,S } \\
\text { LC32A1,SHANK2, } \\
\text { PRKCB,SNAP25,G } \\
\text { ABBR1,GABRA2, } \\
\text { GABRA5,GABRB1 } \\
\text {,GABRB3,GABRG } \\
\text { 2,GAD1,GAD2,CP } \\
\text { LX1,KCNK4,NEFL } \\
\text {,STX1A,STXBP1,P } \\
\text { TPRD,KCNJ4,ABC } \\
\text { C8,KCNJ6,GLS,KC } \\
\text { NMA1,SYN1,SYN } \\
\text { 2,SLC17A7,KCNA } \\
\text { B1,KCNQ2,SYT1, } \\
\text { KCNQ3,KCNS1,D } \\
\text { LG2,DLG4,GNG3, } \\
\text { CACNA1E,CAMK } \\
\text { 2A,CAMK2B,GRI } \\
\text { N1,GRIN2C,SYT7, } \\
\text { KCNH3,EPB41L1 }\end{array}$ \\
\hline 1268766 & $\begin{array}{c}\text { Transmission across Chemical } \\
\text { Synapses }\end{array}$ & $3.25 \mathrm{E}-25$ & $7.88 \mathrm{E}-23$ & 5.33E-22 & $1.58 \mathrm{E}-22$ & 38 & $\begin{array}{c}\text { TSPOAP1,ADCY1, } \\
\text { ADCY2,CACNG3, } \\
\text { PPFIA2,GABBR2,S } \\
\text { LC1A2,SLC6A1,SL } \\
\text { C32A1,PRKCB,SN } \\
\text { AP25,GABBR1,GA } \\
\text { BRA2,GABRA5,G } \\
\text { ABRB1,GABRB3, } \\
\text { GABRG2,GAD1,G } \\
\text { AD2,CPLX1,NEFL, } \\
\text { STX1A,STXBP1,K } \\
\text { CNJ4,KCNJ6,GLS, } \\
\text { SYN1,SYN2,SLC1 } \\
\text { 7A7,SYT1,DLG4,G } \\
\text { NG3,CACNA1E,C } \\
\text { AMK2A,CAMK2B, } \\
\text { GRIN1,GRIN2C,EP }\end{array}$ \\
\hline
\end{tabular}


medRxiv preprint doi: https://doi.org/10.1101/2020.12.21.20248616; this version posted December 23, 2020. The copyright holder for this preprint (which was not certified by peer review) is the author/funder, who has granted medRxiv a license to display the preprint in perpetuity. All rights reserved. No reuse allowed without permission.

Neurotransmitter Release Cycle
$1.56 \mathrm{E}-15$

1268786

1270303

Neurotransmitter Receptor Binding And Downstream Transmission In The

Postsynaptic Cell

Axon guidance
Cardiac conduction

$1.53 \mathrm{E}-07$

$3.15 \mathrm{E}-05$

7.44E-05

15 SCN2A,SCN2B,SC small molecules N8A,FGF12,KCNK 12,SLC8A2,ATP1B 1,ATP2B2,SCN3B, $\mathrm{KCNK} 4, \mathrm{KCNJ} 4, \mathrm{CA}$ MK2A,CAMK2B

B41L1 AD1,GAD2,CPLX ,STX1A,STXBP1,G LS,SYN1,SYN2,SL C17A7,SYT1

ADCY1,ADCY2,C ACNG3,GABBR2, PRKCB,GABBR1, A2,GABRA ,GABRB1,GABRB 3,GABRG2,NEFL, KCNJ4,KCNJ6,DL G4,GNG3,CAMK2 A,CAMK2B,GRIN 1

EPHB6,DOK6,ERB B3,UNC5A,SCN2A ,SCN2B,SCN8A,FG

FR3,RASAL1,SEM

H 3 GL2,SH

B1,DNM3,ABLIM2

,SCN3B,PAK6,NEF

L,CDK5R1,ARHG

EF7,KCNQ2,KCN

Q3,DLG4,CACNA

APIGAP,CAMK2A GRIN2C

$\triangle A C A 2, A S I C 2, A D$

CY1,ADCY2,SLC1

2A5,CLCA4,APOD

,SLC1A2,SLC6A1,

SLC32A1,SLC8A2,

SLCO1A2,ATP1B1

,ATP2B2,ANO3,G

ABRA2,GABRA5,

GABRB1,GABRB3

,GABRG2,ATP6V1

G2,SLC30A3,ABC

C8,SLC17A7,GNG

3,ANO4,CAMK2A,

CAMK2B,SLC6A1

5,TF,SLC7A10,DM 
medRxiv preprint doi: https://doi.org/10.1101/2020.12.21.20248616; this version posted December 23, 2020. The copyright holder for this preprint (which was not certified by peer review) is the author/funder, who has granted medRxiv a license to display the preprint in perpetuity. All rights reserved. No reuse allowed without permission.

TN

1270302

Developmental Biology

8.44E-04 7.97E-03

5.39E-02

4.09E-01

37

EPHB6,DOK6,ERB

B3,STX1B,CACNG

3,UNC5A,SCN2A,S

CN2B,SCN8A,FGF

R3, RASAL1,SEMA

4D,PKP4,SH3GL2,

SHTN1,SHANK3,A

RRB1,LGI3,DNM3,

ABLIM2,SCN3B,P

AK6,NEFL,CDK5R

1,STX1A,ARHGEF

7,KCNQ2,KCNQ3,

DLG4,CACNA1I,D

NM1,CNTN2,RAP1

GAP,CAMK2A,CA

MK2B,GRIN1,GRI

N2C

1269543

Signaling by GPCR

7.06E-03

4.83E-02

3.26E-01

$1.00 \mathrm{E}+00$

40

PDE1A,ERBB3,PD

E2A,PDYN,NTSR2

,ADCY1,CHRM3,A

DCY2,PPP1R1B,F

GFR3,RASAL1,VI

P,VIPR1,HTR2A,P

2RY12,GABBR2,S

1PR5,ARRB1,RTP5

,PRKCB,GABBR1,

RGS11,SST,GPR83

,NEFL,ARHGEF7,

DLG4,GNAO1,GN

G3,NPY,GPR37,CA

MK2A,CAMK2B,G

RIN1,GRIN2C,GR

M3,RGS4,RGS7,C

CK,CCKBR

$1.00 \mathrm{E}+00$

AMPH,PACSIN1,S H3GL2,SH3GL3, R AB6B,ARRB1,NAP B,DNM3,KIF1A,GJ B6,KIAA0319,SNA P91,SYT1,DNM1,D YNC1I1,KLC1,GJC 2,TF,KIFC2,REPS2

\begin{tabular}{|c|c|c|c|c|c|c|c|}
\hline \multicolumn{8}{|c|}{ Gen MAPP } \\
\hline MAP00252 & $\begin{array}{c}\text { Alanine and aspartate } \\
\text { metabolism }\end{array}$ & $8.05 \mathrm{E}-03$ & $5.64 \mathrm{E}-02$ & $1.83 \mathrm{E}-01$ & $1.13 \mathrm{E}-01$ & 3 & $\begin{array}{c}\text { ASPA,GAD1,GAD } \\
2\end{array}$ \\
\hline MAP00512 & O Glycans biosynthesis & $1.49 \mathrm{E}-01$ & $3.48 \mathrm{E}-01$ & $1.00 \mathrm{E}+00$ & $1.00 \mathrm{E}+00$ & 2 & GALNT9 \\
\hline MAP00230 & Purine metabolism & $2.36 \mathrm{E}-01$ & $3.58 \mathrm{E}-01$ & $1.00 \mathrm{E}+00$ & $1.00 \mathrm{E}+00$ & 3 & $\begin{array}{l}\text { PDE1A,ADCY1,A } \\
\text { DCY2 }\end{array}$ \\
\hline MAP00590 & $\begin{array}{c}\text { Prostaglandin and leukotriene } \\
\text { metabolism }\end{array}$ & $3.19 \mathrm{E}-01$ & $3.58 \mathrm{E}-01$ & $1.00 \mathrm{E}+00$ & $1.00 \mathrm{E}+00$ & 1 & PTGDS \\
\hline
\end{tabular}


medRxiv preprint doi: https://doi.org/10.1101/2020.12.21.20248616; this version posted December 23, 2020. The copyright holder for this preprint (which was not certified by peer review) is the author/funder, who has granted medRxiv a license to display the preprint in perpetuity. All rights reserved. No reuse allowed without permission.

\begin{tabular}{|c|c|c|c|c|c|c|c|}
\hline MAP03070 & Type III secretion system & $3.19 \mathrm{E}-01$ & $3.58 \mathrm{E}-01$ & $1.00 \mathrm{E}+00$ & $1.00 \mathrm{E}+00$ & 1 & ATP6V1G2 \\
\hline MAP00562 & Inositol phosphate metabolism & 3.19E-01 & $3.58 \mathrm{E}-01$ & $1.00 \mathrm{E}+00$ & $1.00 \mathrm{E}+00$ & 1 & ITPKA \\
\hline \multicolumn{8}{|c|}{ MSigDB C2 BIOCARTA (v6.0) } \\
\hline M16518 & $\begin{array}{c}\text { Wnt/Ca2+/cyclic GMP } \\
\text { signaling. }\end{array}$ & $5.88 \mathrm{E}-04$ & $1.98 \mathrm{E}-02$ & $1.01 \mathrm{E}-01$ & $5.41 \mathrm{E}-02$ & 4 & $\begin{array}{c}\text { ITPKA,CAMK2A, } \\
\text { CAMK2B,TF }\end{array}$ \\
\hline M7772 & $\begin{array}{l}\text { fl-arrestins in GPCR } \\
\text { Desensitization }\end{array}$ & $8.55 \mathrm{E}-04$ & $1.98 \mathrm{E}-02$ & $1.01 \mathrm{E}-01$ & $7.86 \mathrm{E}-02$ & 3 & $\begin{array}{c}\text { ADCY1,ARRB1,D } \\
\text { NM1 }\end{array}$ \\
\hline M477 & $\begin{array}{l}\text { Gamma-aminobutyric Acid } \\
\text { Receptor Life Cycle }\end{array}$ & $8.55 \mathrm{E}-04$ & $1.98 \mathrm{E}-02$ & $1.01 \mathrm{E}-01$ & $7.86 \mathrm{E}-02$ & 3 & $\begin{array}{c}\text { GABRA2,GABRA5 } \\
\text {,DNM1 }\end{array}$ \\
\hline M11650 & Nitric Oxide SignalingPathway & $8.60 \mathrm{E}-04$ & $1.98 \mathrm{E}-02$ & $1.01 \mathrm{E}-01$ & 7.91E-02 & 4 & $\begin{array}{c}\text { PRKCB,DLG4,GRI } \\
\text { N1,GRIN2C }\end{array}$ \\
\hline M8873 & Fas Signaling Pathway & $1.41 \mathrm{E}-01$ & $5.63 \mathrm{E}-01$ & $1.00 \mathrm{E}+00$ & $1.00 \mathrm{E}+00$ & 3 & $\begin{array}{c}\text { FAIM2,MAPK8IP3, } \\
\text { NCS1 }\end{array}$ \\
\hline M5880 & $\begin{array}{l}\text { Genes encoding proteins } \\
\text { affiliated structurally or } \\
\text { functionally to extracellular } \\
\text { matrix proteins }\end{array}$ & 4.48E-01 & $6.35 \mathrm{E}-01$ & $1.00 \mathrm{E}+00$ & $1.00 \mathrm{E}+00$ & 4 & $\begin{array}{l}\text { ELFN2,C1QTNF4, } \\
\text { PARM1,SEMA4D }\end{array}$ \\
\hline M5884 & $\begin{array}{l}\text { Ensemble of genes encoding } \\
\text { core extracellular matrix } \\
\text { including ECM glycoproteins, } \\
\text { collagens and proteoglycans }\end{array}$ & $8.04 \mathrm{E}-01$ & $8.51 \mathrm{E}-01$ & $1.00 \mathrm{E}+00$ & $1.00 \mathrm{E}+00$ & 4 & $\begin{array}{c}\text { LGI3,RELN,SPOC } \\
\text { K1,NELL1 }\end{array}$ \\
\hline M5883 & $\begin{array}{l}\text { Genes encoding secreted } \\
\text { soluble factors }\end{array}$ & $9.17 \mathrm{E}-01$ & $9.48 \mathrm{E}-01$ & $1.00 \mathrm{E}+00$ & $1.00 \mathrm{E}+00$ & 4 & $\begin{array}{c}\text { FGF12,SFRP1,CCL } \\
\text { 4L2,CBLN2 }\end{array}$ \\
\hline M5885 & $\begin{array}{l}\text { Ensemble of genes encoding } \\
\text { ECM-associated proteins } \\
\text { including ECM-affilaited } \\
\text { proteins, ECM regulators and } \\
\text { secreted factors }\end{array}$ & $9.69 \mathrm{E}-01$ & $9.87 \mathrm{E}-01$ & $1.00 \mathrm{E}+00$ & $1.00 \mathrm{E}+00$ & 9 & $\begin{array}{l}\text { ELFN2,SERPINI1, } \\
\text { FGF12,C1QTNF4,P } \\
\text { ARM1,SEMA4D,S } \\
\text { FRP1,CCL4L2,CBL } \\
\text { N2 }\end{array}$ \\
\hline M5889 & $\begin{array}{c}\text { Ensemble of genes encoding } \\
\text { extracellular matrix and } \\
\text { extracellular matrix-associated } \\
\text { proteins }\end{array}$ & $9.76 \mathrm{E}-01$ & $9.87 \mathrm{E}-01$ & $1.00 \mathrm{E}+00$ & $1.00 \mathrm{E}+00$ & 13 & $\begin{array}{c}\text { ELFN2,SERPINI1, } \\
\text { FGF12,C1QTNF4,P } \\
\text { ARM1,SEMA4D,S } \\
\text { FRP1,CCL4L2,LGI } \\
\text { 3,RELN,SPOCK1,N } \\
\text { ELL1,CBLN2 }\end{array}$ \\
\hline
\end{tabular}

Panther DB

\begin{tabular}{|c|c|c|c|c|c|c|c|}
\hline P05734 & Synaptic vesicle trafficking & $2.84 \mathrm{E}-10$ & $1.45 \mathrm{E}-08$ & $6.54 \mathrm{E}-08$ & $1.45 \mathrm{E}-08$ & 9 & $\begin{array}{l}\text { STX1B,RIMS2,ST } \\
\text { X1A,STXBP1,SYN } \\
\text { 1,SYP,SYT1,SYT7, } \\
\text { UNC13C }\end{array}$ \\
\hline P00042 & $\begin{array}{l}\text { Muscarinic acetylcholine } \\
\text { receptor } 1 \text { and } 3 \text { signaling } \\
\text { pathway }\end{array}$ & $1.13 \mathrm{E}-07$ & $2.89 \mathrm{E}-06$ & $1.31 \mathrm{E}-05$ & $5.79 \mathrm{E}-06$ & 10 & $\begin{array}{l}\text { CHRM3,STX1B,PR } \\
\text { KCB,PRKCZ,SNA } \\
\text { P25,STX1A,KCNQ } \\
\text { 2,GNG3,GRIN1,GR } \\
\text { IN2C }\end{array}$ \\
\hline
\end{tabular}


medRxiv preprint doi: https://doi.org/10.1101/2020.12.21.20248616; this version posted December 23, 2020. The copyright holder for this preprint (which was not certified by peer review) is the author/funder, who has granted medRxiv a license to display the preprint in perpetuity. All rights reserved. No reuse allowed without permission.

\begin{tabular}{|c|c|c|c|c|c|c|c|}
\hline P00037 & $\begin{array}{l}\text { Ionotropic glutamate receptor } \\
\text { pathway }\end{array}$ & $2.56 \mathrm{E}-07$ & 4.03E-06 & $1.82 \mathrm{E}-05$ & $1.31 \mathrm{E}-05$ & 9 & $\begin{array}{l}\text { SLC1A2,SHANK3, } \\
\text { SNAP25,CACNA1 } \\
\text { E,CAMK2A,CAM } \\
\text { K2B,GRIN1,GRIN2 } \\
\text { C,GRM3 }\end{array}$ \\
\hline P00027 & $\begin{array}{l}\text { Heterotrimeric G-protein } \\
\text { signaling pathway-Gq alpha } \\
\text { and Go alpha mediated pathway }\end{array}$ & $3.16 \mathrm{E}-07$ & 4.03E-06 & $1.82 \mathrm{E}-05$ & $1.61 \mathrm{E}-05$ & 13 & $\begin{array}{l}\text { CHRM3,GARNL3, } \\
\text { PRKCB,PRKCZ,R } \\
\text { GS11,KCNJ6,GNA } \\
\text { O1,GNG3,CACNA } \\
\text { 1E,RAP1GAP,GR } \\
\text { M3,RGS4,RGS7 }\end{array}$ \\
\hline P00039 & $\begin{array}{l}\text { Metabotropic glutamate } \\
\text { receptor group III pathway }\end{array}$ & 4.68E-06 & 4.77E-05 & $2.16 \mathrm{E}-04$ & 2.39E-04 & 9 & $\begin{array}{l}\text { STX1B,SLC1A2,S } \\
\text { NAP25,STX1A,SL } \\
\text { C17A7,GNG3,CAC } \\
\text { NA1E,GRIN1,GRI } \\
\text { N2C }\end{array}$ \\
\hline P00029 & Huntington disease & 8.04E-04 & $3.73 \mathrm{E}-03$ & $1.69 \mathrm{E}-02$ & $4.10 \mathrm{E}-02$ & 9 & $\begin{array}{l}\text { TUBB4A,PACSIN1 } \\
\text {,SH3GL3,PRODH, } \\
\text { DLG4,DYNC1I1,C } \\
\text { APN3,GRIN1,GRI } \\
\text { N2C }\end{array}$ \\
\hline P00031 & $\begin{array}{l}\text { Inflammation mediated by } \\
\text { chemokine and cytokine } \\
\text { signaling pathway }\end{array}$ & $3.82 \mathrm{E}-02$ & $7.80 \mathrm{E}-02$ & $3.53 \mathrm{E}-01$ & $1.00 \mathrm{E}+00$ & 8 & $\begin{array}{l}\text { ADCY2,ARRB1,PR } \\
\text { KCB,PRKCZ,PAK6 } \\
\text {,GNG3,CAMK2A,R } \\
\text { GS4 }\end{array}$ \\
\hline P00003 & $\begin{array}{l}\text { Alzheimer disease-amyloid } \\
\text { secretase pathway }\end{array}$ & 4.09E-02 & 8.03E-02 & $3.63 \mathrm{E}-01$ & $1.00 \mathrm{E}+00$ & 4 & $\begin{array}{l}\text { CHRM3,PRKCB,P } \\
\text { RKCZ,KLC1 }\end{array}$ \\
\hline P00021 & FGF signaling pathway & 5.37E-02 & 8.94E-02 & 4.04E-01 & $1.00 \mathrm{E}+00$ & 5 & $\begin{array}{l}\text { FGF12,FGFR3,PPP } \\
\text { 2R2C,PRKCB,PRK } \\
\text { CZ }\end{array}$ \\
\hline P00057 & Wntsignaling pathway & $5.74 \mathrm{E}-01$ & $6.17 \mathrm{E}-01$ & $1.00 \mathrm{E}+00$ & $1.00 \mathrm{E}+00$ & 6 & $\begin{array}{l}\text { SFRP1,ARRB1,PR } \\
\text { KCB,PRKCZ,GNG } \\
\text { 3,CDH18 }\end{array}$ \\
\hline
\end{tabular}

Pathway Ontology

\begin{tabular}{|c|c|c|c|c|c|c|c|}
\hline PW:0000674 & insulin secretion pathway & $2.13 \mathrm{E}-05$ & 7.02E-04 & $2.87 \mathrm{E}-03$ & 7.02E-04 & 5 & $\begin{array}{c}\text { SNAP25,STX1A,S } \\
\text { TXBP1,ABCC8,SY } \\
\text { T7 }\end{array}$ \\
\hline PW:0000027 & glutamate metabolic & $1.95 \mathrm{E}-03$ & $3.22 \mathrm{E}-02$ & $1.32 \mathrm{E}-01$ & $6.43 \mathrm{E}-02$ & 3 & GAD1,GAD2,GLS \\
\hline PW:0000543 & $\begin{array}{c}\text { protein kinase A (PKA) } \\
\text { signaling }\end{array}$ & $1.42 \mathrm{E}-02$ & $1.06 \mathrm{E}-01$ & 4.35E-01 & 4.69E-01 & 4 & $\begin{array}{l}\text { PDE1A,PDE2A,AD } \\
\text { CY1,ADCY2 }\end{array}$ \\
\hline PW:0000448 & neuropeptide $\mathrm{Y}$ metabolic & 2.04E-02 & $1.09 \mathrm{E}-01$ & $4.45 \mathrm{E}-01$ & $6.74 \mathrm{E}-01$ & 1 & NPY \\
\hline PW:0000016 & $\begin{array}{c}\text { amyotrophic lateral sclerosis } \\
\text { disease }\end{array}$ & $3.52 \mathrm{E}-02$ & $1.09 \mathrm{E}-01$ & 4.45E-01 & $1.00 \mathrm{E}+00$ & 2 & SLC1A2,NEFM \\
\hline PW:0000388 & Reelinsignaling & 7.76E-02 & $1.43 \mathrm{E}-01$ & $5.86 \mathrm{E}-01$ & $1.00 \mathrm{E}+00$ & 1 & RELN \\
\hline PW:0000274 & $\begin{array}{l}\text { neuron-to-neuron signaling via } \\
\text { the chemical synapse }\end{array}$ & 7.76E-02 & $1.43 \mathrm{E}-01$ & $5.86 \mathrm{E}-01$ & $1.00 \mathrm{E}+00$ & 1 & $\mathrm{KCNJ} 4$ \\
\hline
\end{tabular}


medRxiv preprint doi: https://doi.org/10.1101/2020.12.21.20248616; this version posted December 23, 2020. The copyright holder for this preprint (which was not certified by peer review) is the author/funder, who has granted medRxiv a license to display the preprint in perpetuity. All rights reserved. No reuse allowed without permission.

\begin{tabular}{|c|c|c|c|c|c|c|c|}
\hline PW:0000243 & $\begin{array}{l}\text { vascular endothelial growth } \\
\text { factor signaling }\end{array}$ & $8.25 \mathrm{E}-02$ & $1.43 \mathrm{E}-01$ & $5.86 \mathrm{E}-01$ & $1.00 \mathrm{E}+00$ & 2 & PRKCB,PRKCZ \\
\hline PW:0000015 & Alzheimer disease & $8.25 \mathrm{E}-02$ & $1.43 \mathrm{E}-01$ & $5.86 \mathrm{E}-01$ & $1.00 \mathrm{E}+00$ & 2 & SNCA,GNAO1 \\
\hline PW:0000579 & somatostatinsignaling & $1.14 \mathrm{E}-01$ & $1.64 \mathrm{E}-01$ & $6.70 \mathrm{E}-01$ & $1.00 \mathrm{E}+00$ & 1 & SST \\
\hline \multicolumn{8}{|c|}{ SMPDB } \\
\hline SMP00246 & Pirenzepine Pathway & $1.56 \mathrm{E}-05$ & $2.55 \mathrm{E}-04$ & $1.37 \mathrm{E}-03$ & $1.88 \mathrm{E}-03$ & 5 & $\begin{array}{l}\text { CHRM3,SNAP25,S } \\
\text { ST,STX1A,CCKBR }\end{array}$ \\
\hline SMP00385 & Homocarnosinosis & $2.04 \mathrm{E}-02$ & $1.88 \mathrm{E}-01$ & $1.00 \mathrm{E}+00$ & $1.00 \mathrm{E}+00$ & 1 & CNDP1 \\
\hline SMP00361 & Hyperprolinemia Type I & $2.04 \mathrm{E}-02$ & $1.88 \mathrm{E}-01$ & $1.00 \mathrm{E}+00$ & $1.00 \mathrm{E}+00$ & 1 & PRODH \\
\hline SMP00067 & Aspartate Metabolism & 2.69E-02 & $2.22 \mathrm{E}-01$ & $1.00 \mathrm{E}+00$ & $1.00 \mathrm{E}+00$ & 2 & ASPA,GAD1 \\
\hline SMP00320 & $\begin{array}{c}\text { Intracellular Signalling } \\
\text { Through Adenosine Receptor } \\
\text { A2a and Adenosine }\end{array}$ & $2.77 \mathrm{E}-02$ & $2.22 \mathrm{E}-01$ & $1.00 \mathrm{E}+00$ & $1.00 \mathrm{E}+00$ & 3 & $\begin{array}{c}\text { ADCY2,PRKCZ,A } \\
\text { RHGEF7 }\end{array}$ \\
\hline SMP00083 & Acetylsalicylic Acid Pathway & 4.45E-02 & $2.66 \mathrm{E}-01$ & $1.00 \mathrm{E}+00$ & $1.00 \mathrm{E}+00$ & 2 & P2RY12,PTGDS \\
\hline SMP00035 & Bile Acid Biosynthesis & $4.45 \mathrm{E}-02$ & $2.66 \mathrm{E}-01$ & $1.00 \mathrm{E}+00$ & $1.00 \mathrm{E}+00$ & 2 & CYP46A1,CH25H \\
\hline SMP00152 & Perindopril Pathway & $1.48 \mathrm{E}-01$ & $2.66 \mathrm{E}-01$ & $1.00 \mathrm{E}+00$ & $1.00 \mathrm{E}+00$ & 2 & ATP1B1,АBCC8 \\
\hline SMP00391 & Insulin Signalling & $1.62 \mathrm{E}-01$ & $2.66 \mathrm{E}-01$ & $1.00 \mathrm{E}+00$ & $1.00 \mathrm{E}+00$ & 2 & PPP1R1A,PRKCZ \\
\hline SMP00048 & $\begin{array}{c}\text { Nicotinate and Nicotinamide } \\
\text { Metabolism }\end{array}$ & $2.15 \mathrm{E}-01$ & $2.66 \mathrm{E}-01$ & $1.00 \mathrm{E}+00$ & $1.00 \mathrm{E}+00$ & 1 & NMNAT2 \\
\hline
\end{tabular}

Table 4 The enriched GO terms of the up regulated differentially expressed genes

\begin{tabular}{|c|c|c|c|c|c|c|c|c|}
\hline GO ID & CATEGORY & GO Name & $P$ Value & FDR B\&H & $\begin{array}{l}\text { FDR } \\
\text { B\&Y }\end{array}$ & Bonferroni & $\begin{array}{l}\text { Gene } \\
\text { Count }\end{array}$ & Gene \\
\hline GO:0009057 & $\mathrm{BP}$ & $\begin{array}{c}\text { macromolec } \\
\text { ule catabolic } \\
\text { process }\end{array}$ & $1.79 \mathrm{E}-15$ & $5.08 \mathrm{E}-13$ & $4.66 \mathrm{E}-12$ & $9.66 \mathrm{E}-12$ & 72 & $\begin{array}{c}\text { RPL23A,RPL29,P } \\
\text { TTG1,RPL31,RPL } \\
\text { P1,RPS2,RPS3,RP } \\
\text { S3A,RPS4X,RPS4 } \\
\text { Y1,RPS7,RPS8,RP } \\
\text { S10,RPS11,RPS12 } \\
\text {,RPS13,RPS15A,H } \\
\text { MGB1,HMGB2,R } \\
\text { PS18,RPS25,RPS2 } \\
\text { 6,RPS27,HMMR, } \\
\text { RPS27A,RPS28,R } \\
\text { PS29,CHI3L2,LS } \\
\text { M5,HNRNPC,RP } \\
\text { L23,HSPA1A,CP } \\
\text { VL,VEGFA,VIM, } \\
\text { ANXA2,PPP2CB,I } \\
\text { GF2BP3,IGFBP3, } \\
\text { UHRF1,SUMO2,C } \\
\text { YP51A1,PBK,AU } \\
\text { RKA,BNIP3L,FB } \\
\text { XO5,ZFP36L1,AD }\end{array}$ \\
\hline
\end{tabular}


medRxiv preprint doi: https://doi.org/10.1101/2020.12.21.20248616; this version posted December 23, 2020. The copyright holder for this preprint (which was not certified by peer review) is the author/funder, who has granted medRxiv a license to display the preprint in perpetuity. All rights reserved. No reuse allowed without permission.

GO:0000278

GO:0007049
BP

mitotic cell

BP

cell cycle cycle

4.62E-15

$1.08 \mathrm{E}-12$

9.95E- 12

2.49E-11

(n)

2.88E-14

5.37E-12

4.93E-11

$1.56 \mathrm{E}-10$
AMTS9,PYGL,U BE2C,CAST,RPL 14,CAV1,EEF1A1 ,CCNB1,USP8,RN ASE2,TIMP1,TIM P4,CD44,CDK1,L UM,RPL6,RPL7,R PL7A,RPL9,MAD 2L1,PCNA,CDK2, RPL15,AURKB,R

PL18A

NUSAP1,CDKN3, PTTG1,CENPW,C ENPA,CENPF,H MGB1,HMMR,TT K,CDC45,CKS2,G PNMB,HSPA1A,P HLDA1, WEE1,A NXA1,ID2,ID4,BI RC5,PDPN,TPX2, CDCA5,MYC,NE S,DLGAP5,PBK, MELK,CENPK,A URKA,TUBB6,PT TG3P,NEK2,NCA PG,FBXO5,ZFP36 L1,BUB1,BTG3,K IF11,KIFC1,RAN, TUBA1C,UBE2C, PRC1,ECT2,GMN N,CCNA2,CCNB1 ,CEP55,KIF20A,U SP8,CCNB2,SBD S,EIF4E,CDK1,C KAP2,TOP2A,MA D2L1,PCNA,CDK 2,AURKB

NUSAP1,CDKN3, PTTG1,CENPW,C ENPA,CENPF,RP S3,HJURP,HLAG,ETS1,RPS15A, HMGB1,OIP5,HM MR,TTK,CDC45, RPL23,CKS2,CLI C1,ASPM,PIMRE G,GPNMB,HSPA 1A,PHLDA1,WEE 1,ANXA1,MCTS1 ,ID2,ID3,ID4,BIR C5,WTAP,PDPN, TPX2,MPLKIP,U HRF1,CDCA5,CX CL8,MYC,NES,P CLAF,DLGAP5, T CIM,PBK,MELK, CENPK,NEDD9, AURKA,TUBB6, PTTG3P,NEK2,N 
medRxiv preprint doi: https://doi.org/10.1101/2020.12.21.20248616; this version posted December 23, 2020. The copyright holder for this preprint (which was not certified by peer review) is the author/funder, who has granted medRxiv a license to display the preprint in perpetuity. All rights reserved. No reuse allowed without permission.

GO:0010941

GO:0071345
BP

regulation of cell death
4.12E-09 3.78E-08 $1.77 \mathrm{E}-07$

(1)

CAPG,FBXO5,ZF

P36L1,BUB1,BTG

3,SLBP,KIF11,KI

FC1,RAN,TUBA1

C,UBE2C,CAST,E

2F2,PRC1,ECT2,

GMNN,ACTR3,C

CNA2,CCNB1,CE

P55,KIF20A,USP8

CCNB2,SBDS,EI

F4E,STEAP3,CD

K1,CKAP2,TOP2

A,MAD2L1,PCN

A,CDK2,AURKB

TPT1,HIF1A,HEB

P2,RPS3,RPS3A,R

PS7,HLA-

G,ETS1,HMGB1, HMGB2,RPS27A,

HMOX1,RPS29,M DK,F2R,F3,HNR

NPK,TNFRSF12A

,HOXA5,ADM,HP ,ZFAND6,SRPX,

GPNMB,MMP9,H

SPA1A,HSPA4,N

SMAF,VEGFA,H

SPD1,HSPE1,PHL

DA1,ANXA1,GD

F15,ID3,NOX4,BI RC5,SLC40A1,A

KAP12,PDPN,IGF BP3,GNA13,PTG ES3,SOD2,MYC, NES,GAPDH,TCI M,ITGA5,MELK, PTMA,SOCS2,AU RKA,TSC22D1,B NIP3L,ZFP36L1,B UB1,FAM162A,C AST,CASP4,CAV 1,ECT2,TFAP2A, LGALS1,LGALS3 ,EEF1A1,APH1A, TIMP1,LOX,IER3 IP1,CD44,STEAP 3,CDK1,TOP2A,A LPK2,MAD2L1,A URKB

HIF1A,HLAA,RPS2,RPS3,HL A-G,HLAH,HMGB1,RPS27 A,HMOX1,CHI3L 1,F3,F13A1,TNFR SF12A,SAA1,ZFA ND6,IFI30,TNFR SF19,CCL20,MM P9,HSPA1A,VCA 
medRxiv preprint doi: https://doi.org/10.1101/2020.12.21.20248616; this version posted December 23, 2020. The copyright holder for this preprint (which was not certified by peer review) is the author/funder, who has granted medRxiv a license to display the preprint in perpetuity. All rights reserved. No reuse allowed without permission.

\begin{tabular}{|c|c|c|c|c|c|c|c|c|}
\hline GO:0030334 & $\mathrm{BP}$ & $\begin{array}{l}\text { regulation of } \\
\text { cell } \\
\text { migration }\end{array}$ & 7.44E-09 & $5.09 \mathrm{E}-07$ & 4.67E-06 & $4.02 \mathrm{E}-05$ & 47 & $\begin{array}{l}\text { HIF1A,ETS1,HM } \\
\text { GB1,HMOX1,MD } \\
\text { K,F2R,F3,CD99,P } \\
\text { LAU,GPNMB,CC } \\
\text { L20,MMP9,COL1 } \\
\text { A1,VEGFA,COL3 } \\
\text { A1,VIM,ANGPT2, } \\
\text { ANXA1,NOX4,C } \\
\text { LIC4,AKAP12,PD } \\
\text { PN,POSTN,IGFB } \\
\text { P3,PRCP,GNA13, } \\
\text { SOD2,CXCL8,M } \\
\text { YC,ITGA5,STC1, } \\
\text { NEDD9,SRPX2,F } \\
\text { BXO5,ADAMTS9 } \\
\text {,BST2,TMSB15A, } \\
\text { TMSB15B,LAMA } \\
\text { 4,LAMB1,CAV1, } \\
\text { TFAP2A,ACTR3, } \\
\text { LGALS3,TIMP1,T } \\
\text { MSB10,TNFAIP6 }\end{array}$ \\
\hline GO:0072359 & $\mathrm{BP}$ & $\begin{array}{c}\text { circulatory } \\
\text { system } \\
\text { development }\end{array}$ & $1.15 \mathrm{E}-08$ & $7.22 \mathrm{E}-07$ & $6.62 \mathrm{E}-06$ & $6.21 \mathrm{E}-05$ & 54 & $\begin{array}{c}\text { TSPAN12,HIF1A, } \\
\text { HLA- } \\
\text { G,ETS1,HMGB1, } \\
\text { HMOX1,CHI3L1, } \\
\text { MDK,F3,TNFRSF } \\
\text { 12A,HOXA5,AD } \\
\text { M,PLAU,GPNMB } \\
\text {,MMP9,VCAM1,C } \\
\text { OL1A1,COL1A2, } \\
\text { VEGFA,COL3A1, } \\
\text { COL4A1,COL5A1 } \\
\text {,COL8A1,PRRX1, } \\
\text { ANGPT2,ANXA1, } \\
\text { ANXA2,ID2,ID3, } \\
\text { NOX4,CLIC4,YY } \\
\text { 1,PDPN,PRCP,GN } \\
\text { A13,SOD2,CXCL } \\
\text { 8,ITGA5,SRPX2,Z } \\
\text { FP36L1,ADAMTS } \\
\text { 9,LAMA4,ESM1, } \\
\text { E2F2,CAV1,TFAP } \\
\text { 2A,LGALS3,CCN } \\
\text { B1,TGFBI,PDLIM }\end{array}$ \\
\hline
\end{tabular}


medRxiv preprint doi: https://doi.org/10.1101/2020.12.21.20248616; this version posted December 23, 2020. The copyright holder for this preprint (which was not certified by peer review) is the author/funder, who has granted medRxiv a license to display the preprint in perpetuity. All rights reserved. No reuse allowed without permission.

\begin{tabular}{|c|c|c|c|c|c|c|c|c|}
\hline & & & & & & & & $\begin{array}{c}\text { 1,LOX,CDK1,AL } \\
\text { PK2,PCNA }\end{array}$ \\
\hline GO:0048646 & $\mathrm{BP}$ & $\begin{array}{l}\text { anatomical } \\
\text { structure } \\
\text { formation } \\
\text { involved in } \\
\text { morphogenes } \\
\text { is }\end{array}$ & 4.83E-07 & $1.98 \mathrm{E}-05$ & $1.81 \mathrm{E}-04$ & $2.61 \mathrm{E}-03$ & 51 & $\begin{array}{c}\text { TSPAN12,HIF1A, } \\
\text { RPS7,HLA- } \\
\text { G,ETS1,HMGB1, } \\
\text { HMOX1,CHI3L1, } \\
\text { MDK,WLS,F3,TN } \\
\text { FRSF12A,HOXA5 } \\
\text {,ADM,CTHRC1,P } \\
\text { LAU,GPNMB,M } \\
\text { MP9,COL1A1,VE } \\
\text { GFA,COL4A1,CO } \\
\text { L5A1,COL5A2,C } \\
\text { OL8A1,ANGPT2, } \\
\text { ANXA1,ANXA2, } \\
\text { GDF15,CLIC4,IR } \\
\text { X3,SLC40A1,PDP } \\
\text { N,PRCP,GNA13,S } \\
\text { F3B6,CSRP2,CXC } \\
\text { L8,SP3,ITGA5,SR } \\
\text { PX2,ADAMTS9,R } \\
\text { DH10,LAMB1,ES } \\
\text { M1,E2F2,CAV1,T } \\
\text { FAP2A,LGALS3, } \\
\text { TGFBI,TGIF1,CD } \\
44\end{array}$ \\
\hline GO:0009628 & $\mathrm{BP}$ & $\begin{array}{l}\text { response to } \\
\text { abiotic } \\
\text { stimulus }\end{array}$ & $6.01 \mathrm{E}-07$ & $2.35 \mathrm{E}-05$ & $2.16 \mathrm{E}-04$ & $3.25 \mathrm{E}-03$ & 51 & $\begin{array}{c}\text { HIF1A,ETS1,HM } \\
\text { GN1,RPS27A,HM } \\
\text { OX1,CHI3L1,MD } \\
\text { K,ADM,AK4,PLA } \\
\text { U,MMP7,HILPDA } \\
\text {,HSPA1A,PLOD2, } \\
\text { HSPA4,VCAM1,C } \\
\text { OL1A1,VEGFA,C } \\
\text { OL3A1,HSPD1,A } \\
\text { NGPT2,TNC,AN } \\
\text { XA1,ID2,NOX4,Y } \\
\text { Y1,AKAP12,POS } \\
\text { TN,IGFBP2,STC2, } \\
\text { PTGES3,SOD2,M } \\
\text { YC,PCLAF,TCIM, } \\
\text { PBK,STC1,BNIP3 } \\
\text { L,ZFP36L1,FAM1 } \\
\text { 62A,CA9,CAV1,E } \\
\text { CT2,CCNA2,CCN } \\
\text { B1,NAMPT,PDLI } \\
\text { M1,LPL,PCNA,C } \\
\text { DK2,AURKB }\end{array}$ \\
\hline GO:0048870 & $\mathrm{BP}$ & cell motility & $1.00 \mathrm{E}-06$ & $3.66 \mathrm{E}-05$ & $3.36 \mathrm{E}-04$ & $5.41 \mathrm{E}-03$ & 64 & $\begin{array}{l}\text { HIF1A,ETS1,HM } \\
\text { GB1,HMGB2,HM } \\
\text { OX1,MDK,F2R,F } \\
\text { 3,TNFRSF12A,H } \\
\text { OXA5,SAA1,CD9 } \\
\text { 9,ASPM,CTHRC1 } \\
\text {,PLAU,GPNMB,C } \\
\text { CL20,MMP9,VCA } \\
\text { M1,COL1A1,COL } \\
\text { 1A2,VEGFA,COL }\end{array}$ \\
\hline
\end{tabular}


medRxiv preprint doi: https://doi.org/10.1101/2020.12.21.20248616; this version posted December 23, 2020. The copyright holder for this preprint (which was not certified by peer review) is the author/funder, who has granted medRxiv a license to display the preprint in perpetuity. All rights reserved. No reuse allowed without permission.

GO:0044445

GO:0062023
$\mathrm{CC}$

$\mathrm{CC}$

$$
\begin{gathered}
\text { collagen- } \\
\text { containing } \\
\text { extracellular } \\
\text { matrix }
\end{gathered}
$$

$6.23 \mathrm{E}-21$

1.05E-18

7.42E-18

part
3A1,VIM,COL5A

1,ANGPT2,ANXA

1,NOX4,CLIC4,P

PIA,AKAP12,PDP

N,POSTN,IGFBP3

,PRCP,GNA13,SO

D2,CXCL8,MYC,I

TGA5,STC1,NED

D9,SRPX2,FBXO

5,ADAMTS9,BST

2,RAB13,TMSB1

5A,TMSB15B,LA

MA4,LAMB1,LA

MC1,CAV1,TFAP

2A,ACTR3,LGAL

S3,SLC16A3,TIM

P1,SBDS,LOX,CD

44,TMSB10,CDK

1,TNFAIP6

RPL23A,RPL29, R

PL31,RPLP1,RPS

2,RPS3,RPS3A,RP

S4X,RPS4Y1,RPS

7,RPS8,RPS10,RP

S11,RPS12,RPS13

,RPS15A,RPS18,R

PS25,RPS26,RPS2

7,RPS27A,RPS28,

RPS29,RPL23,EIF

3M,MCTS1,CCT8

,LARP4,CASP4,R

PL14,EIF4E,RPL6

,RPL7,RPL7A,RP

L9,RPL15,RPL18

A

$\begin{array}{lllll}2.26 \mathrm{E}-19 & 3.04 \mathrm{E}-17 & 2.16 \mathrm{E}-16 & 1.52 \mathrm{E}-16 & 44\end{array}$ EMILIN2,MDK,F

3,F13A1,S100A3,

S100A4,S100A6,S

100A10,S100A11,

MGP,AEBP1,CTH

RC1,SRPX,MMP9

,COL1A1,COL1A

2,VEGFA,COL3A

1,COL4A1,COL5

A1,COL5A2,COL

6A3,COL8A1,MX

RA5,TNC,ANXA

1,ANXA2,GDF15,

POSTN,SRPX2,A

DAMTS9,LAMA4

,LAMB1,LAMC1,

SERPINH1,LGAL

S1,LGALS3,TGF

BI,TIMP1,LOX,P

COLCE2,LUM

GO:0005912

CC

adherens

7.82E-14

4.38E-12

3.10E-11

5.25E-11

40

RPL31,RPLP1,RP S2,RPS3,RPS3A,R 
medRxiv preprint doi: https://doi.org/10.1101/2020.12.21.20248616; this version posted December 23, 2020. The copyright holder for this preprint (which was not certified by peer review) is the author/funder, who has granted medRxiv a license to display the preprint in perpetuity. All rights reserved. No reuse allowed without permission.

junction

PS4X,RPS7,RPS8, RPS10,RPS11,RP S13,RPS18,RPS29 ,HNRNPK,RPL23, S100A11,CD99,P LAU,HSPA1A,VE GFA,VIM,TNC,A NXA1,ANXA2,N OX4,PPIA,AKAP 12,GNA13,CSRP2 ,ITGA5,NEDD9,C AV1,ACTR3,G3B P1,PDLIM1,CD44 ,RPL6,RPL7,RPL7

A,RPL9

GO:0030141

$\mathrm{CC}$

$$
\begin{aligned}
& \text { secretory } \\
& \text { granule }
\end{aligned}
$$

2.18E-08

6.11E-07

4.33E-06

1.47E-05

cytoplasmic
vesicle part

$1.82 \mathrm{E}-07$

4.21E-06

2.99E-05

$1.22 \mathrm{E}-04$

\begin{abstract}
CC
\end{abstract}

(


medRxiv preprint doi: https://doi.org/10.1101/2020.12.21.20248616; this version posted December 23, 2020. The copyright holder for this preprint (which was not certified by peer review) is the author/funder, who has granted medRxiv a license to display the preprint in perpetuity. All rights reserved. No reuse allowed without permission.

GO:0099081

GO:0015630

GO:0009986
$\mathrm{CC}$

\begin{abstract}
supramolecul ar polymer
\end{abstract}

8.36E-06
$6, \mathrm{LYZ}$

NUSAP1,TPT1,C
ENPF,HLA-

G,ACTG2,SAA1, CLIC1,ASPM,VC AM1,COL1A1,CO L1A2,COL3A1,C OL4A1,VIM,COL 5A1,COL5A2,CO L6A3,COL8A1,A NXA1,ANXA2,N OX4,CLIC4,BIRC 5,PDPN,GNA13,C SRP2,TPX2,CCT8 ,PTGES3,PRPH,N ES,POTEKP,DBI, AURKA,TUBB6, NEK2,KIF11,KIF C1,CA9,RAN,TU BA1C,PRC1,ACT R3,KIF20A,RHO Q,PDLIM1,TIMP4 ,CD44,CDK1,CK AP2,LUM,RPL6,R PL7,RPL15,AUR

$\mathrm{KB}$

$\begin{array}{llllll}\text { microtubule } & 5.06 \mathrm{E}-06 & 7.39 \mathrm{E}-05 & 5.24 \mathrm{E}-04 & 3.40 \mathrm{E}-03 & 48\end{array}$ cytoskeleton

cell surface

4.52E-04

4.53E-03
3.04E-01
NUSAP1,TPT1,C ENPF,RPS3,RPS7, HMMR,TTK,CDC 45,SAA1,ASPM, R ELL1,HSPA1A,D CAF13,CLIC4,BI RC5,PPP2CB,TPX 2,MPLKIP,CCT8, MYC,GAPDH,PC LAF,DLGAP5,NE DD9,AURKA,TU BB6,NEK2,FBXO 5,KIF11,KIFC1,R AN,TUBA1C,PRC 1,ECT2,TFAP2A, CCNB1,CEP55,KI F20A,CCNB2,SB DS,CKAP2L,CDK 1,CKAP2,TOP2A, MAD2L1,PCNA, CDK2,AURKB

HLA-A,HLAG,HLAH,HMGB1,HMM R,F2R,F3,CD163, TNFRSF12A,LIP G,PLAU,SRPX,M MP7,HILPDA,VC AM1,VEGFA,HS PD1,ANXA1,AN XA2,CLIC4,CD93 ,PDPN,CLEC2D,I 
medRxiv preprint doi: https://doi.org/10.1101/2020.12.21.20248616; this version posted December 23, 2020. The copyright holder for this preprint (which was not certified by peer review) is the author/funder, who has granted medRxiv a license to display the preprint in perpetuity. All rights reserved. No reuse allowed without permission.

\begin{tabular}{|c|c|c|c|c|c|c|c|c|}
\hline & & & & & & & & $\begin{array}{c}\text { L13RA2,ITGA5,S } \\
\text { RPX2,ADAMTS9, } \\
\text { BST2,CAV1,LGA } \\
\text { LS1,LGALS3,TFP } \\
\text { I,LPL,CD44 }\end{array}$ \\
\hline GO:0048471 & $\mathrm{CC}$ & $\begin{array}{l}\text { perinuclear } \\
\text { region of } \\
\text { cytoplasm }\end{array}$ & $1.81 \mathrm{E}-03$ & $1.40 \mathrm{E}-02$ & $9.89 \mathrm{E}-02$ & $1.00 \mathrm{E}+00$ & 27 & $\begin{array}{c}\text { CDKN3,CENPF,H } \\
\text { MGB2,HMOX1,C } \\
\text { HI3L1,S100A4,S1 } \\
\text { 00A6,CLIC1,HSP } \\
\text { A1A,VIM,ANXA } \\
\text { 2,NOX4,CLIC4,S } \\
\text { TC2,PTGES3,MY } \\
\text { C,GAPDH,PCLAF } \\
\text {,GBP2,DBI,NXT2, } \\
\text { AURKA,LAMB1, } \\
\text { CAV1,ACTR3,EI } \\
\text { F4E,MAD2L1 }\end{array}$ \\
\hline GO:0098805 & $\mathrm{CC}$ & $\begin{array}{c}\text { whole } \\
\text { membrane }\end{array}$ & $2.07 \mathrm{E}-02$ & $9.04 \mathrm{E}-02$ & $6.41 \mathrm{E}-01$ & $1.00 \mathrm{E}+00$ & 43 & $\begin{array}{c}\text { HLA- } \\
\text { A,RPN2,HLA- } \\
\text { G,HLA- } \\
\text { H,RPS27A,HMO } \\
\text { X1,LY96,WLS,F2 } \\
\text { R,CD163,FABP5, } \\
\text { S100A10,EVA1A, } \\
\text { BRI3,PLAU,GPN } \\
\text { MB,HSPA1A,HSP } \\
\text { D1,ANXA1,ANX } \\
\text { A2,CD93,PDPN,C } \\
\text { CZ1,PRCP,ABCC } \\
\text { 3,CD164,SEC24D, } \\
\text { BNIP3L,BST2,RA } \\
\text { B13,KDELR2,RA } \\
\text { P1B,CAV1,SERPI } \\
\text { NH1,LGALS3,EE } \\
\text { F1A1,TFPI,RHOQ } \\
\text {,USP8,APH1A,CD } \\
\text { 44,CD68,STEAP3 }\end{array}$ \\
\hline GO:0005198 & MF & $\begin{array}{l}\text { structural } \\
\text { molecule } \\
\text { activity }\end{array}$ & $3.13 \mathrm{E}-24$ & $2.93 \mathrm{E}-21$ & $2.17 \mathrm{E}-20$ & $2.93 \mathrm{E}-21$ & 65 & $\begin{array}{c}\text { RPL23A,RPL29,R } \\
\text { PL31,RPLP1,RPS } \\
\text { 2,RPS3,RPS3A,RP } \\
\text { S4X,RPS4Y1,RPS } \\
\text { 7,RPS8,RPS10,RP } \\
\text { S11,RPS12,RPS13 } \\
\text {,RPS15A,EMILIN } \\
\text { 2,RPS18,RPS25,R } \\
\text { PS26,RPS27,RPS2 } \\
\text { 7A,RPS28,RPS29, } \\
\text { CHI3L1,RPL23,M } \\
\text { GP,AEBP1,CTHR } \\
\text { C1,SRPX,COL1A } \\
\text { 1,COL1A2,COL3 } \\
\text { A1,COL4A1,VIM, } \\
\text { COL5A1,COL5A2 } \\
\text {,COL6A3,COL8A } \\
\text { 1,MXRA5,TNC,A } \\
\text { NXA1,POSTN,CS } \\
\text { RP2,PRPH,POTE } \\
\text { KP,TUBB6,SRPX }\end{array}$ \\
\hline
\end{tabular}


medRxiv preprint doi: https://doi.org/10.1101/2020.12.21.20248616; this version posted December 23, 2020. The copyright holder for this preprint (which was not certified by peer review) is the author/funder, who has granted medRxiv a license to display the preprint in perpetuity. All rights reserved. No reuse allowed without permission.

GO:0003723

GO:0005102
MF

signaling

2.04E-07

binding
2,TUBA1C,LAM A4,LAMB1,LAM C1,RPL14,CAV1, ACTR3,RNA28S N5,TGFBI,PCOL CE2,LUM,RPL6,R PL7,RPL7A,RPL9

,RPL15,RPL18A

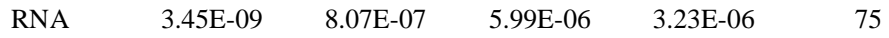
binding (3.

$8.07 \mathrm{E}-07-2.99 \mathrm{E}-06-23 \mathrm{E}-06$

NUSAP1,RPL23A ,TPT1,RPL29,RPL 31,HLAA,RPS2,RPS3,RP S3A,RPS4X,RPS4 Y1,RPS7,RPS8,RP S10,RPS11,RPS12 ,RPS13,RPS15A,H MGB1,HMGB2,R PS18,RPS25,RPS2 6,RPS27,RPS27A, RPS28,HNRNPA1 ,LSM5,HNRNPC, HNRNPK,S100A4 ,RPL23,HSPA1A, EIF3M,HSPD1,VI M,HSPE1,DCAF1 3,ANXA1,ANXA 2,TENT5A,MCTS 1,PPIA,YY1,CNB P,IGF2BP3,SF3B6 ,SUMO2,SNRPG, MYC,LARP4,ZFP 36L1,BST2,SLBP, KPNA2,RAN,CA ST,RPL14,SERPI NH1,LGALS1,LG ALS3,EEF1A1,EI F1AY,EEF1B2,G3 BP1,SLC16A3,SB DS,EIF4E,RPL6,T OP2A,RPL7,RPL7 A,RPL9,RPL15,R PL18A

A2M,CMTM3,HI F1A,HLAA,HLA-G,HLAH,HMGB 1,HMGB 2,LY96,MDK,WL S,F2R,S100A4,AD M,SAA1,CTHRC1 ,GPNMB,CCL20, MMP9,HILPDA,H SPA1A,VCAM1, NSMAF, VEGFA, COL3A1,COL5A1 ,DCAF13,ANGPT 2,ANXA1,ANXA 2,GDF15,PDPN,I GFBP2,IGFBP4,S TC2,CNIH4,GNA 
medRxiv preprint doi: https://doi.org/10.1101/2020.12.21.20248616; this version posted December 23, 2020. The copyright holder for this preprint (which was not certified by peer review) is the author/funder, who has granted medRxiv a license to display the preprint in perpetuity. All rights reserved. No reuse allowed without permission.

\begin{tabular}{|c|c|c|c|c|c|c|c|c|}
\hline GO:0044877 & MF & $\begin{array}{l}\text { protein- } \\
\text { containing } \\
\text { complex } \\
\text { binding }\end{array}$ & $4.48 \mathrm{E}-07$ & $5.24 \mathrm{E}-05$ & $3.88 \mathrm{E}-04$ & 4.19E-04 & 53 & $\begin{array}{c}\text { RPL23A,PTTG1, } \\
\text { HIF1A,RPLP1,CE } \\
\text { NPA,CENPF,RPN } \\
\text { 2,RPS2,RPS3,HM } \\
\text { GB1,HMGN1,F2R } \\
\text {,TTK,HNRNPC,H } \\
\text { NRNPK,AEBP1,C } \\
\text { 1QTNF1,GPNMB, } \\
\text { UQCRH,MMP9,H } \\
\text { SPA4,VCAM1,CO } \\
\text { L3A1,HSPD1,VI } \\
\text { M,COL5A1,ANX } \\
\text { A2,CLIC4,GNA13 } \\
\text {,UHRF1,CDCA5,S } \\
\text { NRPG,MYC,NES, } \\
\text { ITGA5,FBXO5,R } \\
\text { AN,RAP1B,LAM } \\
\text { B1,ESM1,CAV1,S } \\
\text { ERPINH1,ACTR3 } \\
\text {,LGALS3,CCNB1, } \\
\text { TGFBI,SBDS,LPL } \\
\text {,CD44,PCOLCE2, } \\
\text { LUM,PCNA,CDK } \\
2\end{array}$ \\
\hline GO:0008233 & MF & $\begin{array}{l}\text { peptidase } \\
\text { activity }\end{array}$ & $1.35 \mathrm{E}-04$ & $5.18 \mathrm{E}-03$ & $3.84 \mathrm{E}-02$ & $1.27 \mathrm{E}-01$ & 44 & $\begin{array}{c}\text { A2M,SERPINA3, } \\
\text { PTTG1,HIF1A,RP } \\
\text { S3,HMGB1,RPS2 } \\
\text { 7A,F2R,F3,AEBP } \\
\text { 1,HP,PLAU,MMP } \\
\text { 7,MMP9,CPVL,V } \\
\text { EGFA,HSPD1,HS } \\
\text { PE1,COL6A3,BIR } \\
\text { C5,CFI,YY1,PRC } \\
\text { P,MYC,GAPDH,G } \\
\text { GH,ADAMTS9,B } \\
\text { ST2,C1S,FAM162 } \\
\text { A,CAST,CASP4,C } \\
\text { AV1,PRSS23,SER } \\
\text { PINH1,CCNA2,T } \\
\text { FPI,USP8,APH1A, } \\
\text { TIMP1,TIMP4,CD } \\
\text { 44,PCOLCE2,CD } \\
\text { K1 }\end{array}$ \\
\hline GO:0042802 & MF & $\begin{array}{c}\text { identical } \\
\text { protein }\end{array}$ & 4.03E-04 & $1.11 \mathrm{E}-02$ & $8.23 \mathrm{E}-02$ & 3.77E-01 & 59 & $\begin{array}{l}\text { CENPF,HJURP,H } \\
\text { LA- } \\
\text { G,ETS1,OIP5,HM }\end{array}$ \\
\hline
\end{tabular}


medRxiv preprint doi: https://doi.org/10.1101/2020.12.21.20248616; this version posted December 23, 2020. The copyright holder for this preprint (which was not certified by peer review) is the author/funder, who has granted medRxiv a license to display the preprint in perpetuity. All rights reserved. No reuse allowed without permission.

binding

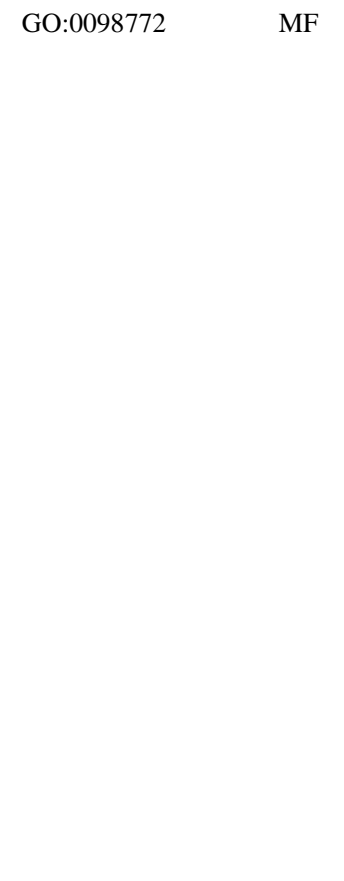

GO:0046983

MF

\begin{abstract}
molecular
function
\end{abstract}

regulator

protein dimerization activity
OX1,TTK,HNRN PC,HNRNPK,FA BP5,S100A4,S100 A6,S100A10,S100 A11,HP,BRI3,MM P9,COL1A1,COL 1A2,VEGFA,VIM, ANXA1,ANXA2, GDF15,PRDX4,BI RC5,SLC40A1,ST C2,FTH1,UHRF1, SOD2,UAP1,GAP DH,GBP1,GBP2,B CHE,SRPX2,BNI P3L,BST2,PYGL, SLBP,C1S,CAV1, PRC1,PAPSS1,EC T2,TFAP2A,LGA LS1,LGALS3,NA MPT,P4HA1,LPL, STEAP3,LYZ,TO P2A,RPL7,MAD2 L1,PCNA,TPI $\begin{array}{lllll}1.14 \mathrm{E}-03 \quad 2.53 \mathrm{E}-02 & 1.88 \mathrm{E}-01 \quad 1.00 \mathrm{E}+00 & 55\end{array}$ A2M,SERPINA3, PTTG1,CMTM3,R PLP1,RPS7,HMG B1,HMGB2,CHI3 L1,MDK,HNRNP C,RPL23,ADM,C KS2,SAA1,GPNM B,CCL20,NSMAF ,VEGFA,COL6A3 ,ANXA1,ANXA2, GDF15,BIRC5,AP OC1,IGFBP3,CCZ 1,STC2,SLN,TSP AN13,CXCL8,GA PDH,STC1,SOCS 2,LYPD1,FBXO5, BST2,NMB,MCU B,CAST,CAV1,E CT2,SERPINH1,P PP1R14B,LGALS 3,CCNA2,CCNB1, TFPI,EEF1B2,NA MPT,TIMP1,TIM P4,CCNB2,PCOL CE2,PCNA

HIF1A,CENPW,C ENPA,CENPF,HL A-

G,HMOX1,TTK,L SM5,S100A6,S10 0A10,S100A11,H P,VEGFA,HSPD1, ANXA1,GDF15,P RDX4,ID2,ID3,ID 4,BIRC5,STC2,PR PH,MYC,GAPDH, 
medRxiv preprint doi: https://doi.org/10.1101/2020.12.21.20248616; this version posted December 23, 2020. The copyright holder for this preprint (which was not certified by peer review) is the author/funder, who has granted medRxiv a license to display the preprint in perpetuity. All rights reserved. No reuse allowed without permission.

GO:0016772

GO:0042803
MF

MF
4.91E-03

$6.28 \mathrm{E}-02$

4.66E-01

$1.00 \mathrm{E}+00$
transferase activity, transferring phosphorus- containing groups

7.69E-02

$5.70 \mathrm{E}-0$
$1.00 \mathrm{E}+00$
51

31
GBP1,GBP2,DBI, AURKA,BNIP3L, BST2,PYGL,RAN ,E2F2,CAV1,PAP SS1,ECT2,TFAP2 A,LGALS1,LGAL S3,NAMPT,LPL, T OP2A,RPL7,MAD

2L1,TPI

CDKN3,HIF1A,R PLP1,RPS3,HMG B1,RPS27A,CHI3 L1,F2R,TTK,HNR NPA1,HNRNPC,C KS2,SAA1,CLK1, AK4,GPNMB,VE GFA,WEE1,GDF1 5,TENT5A,NOX4 TPX2,CCT8,PTG ES3,CXCL8,MYC ,UAP1,GAPDH,T CIM,PBK,MELK, NEDD9,SOCS2,A URKA,NEK2,CH PT1,BUB1,CAV1, PAPSS1,ECT2,CC NA2,EEF1A1,CC NB1,CCNB2,CD4 4,CDK1,TOP2A,A LPK2,CDK2,AUR $\mathrm{KB}, \mathrm{TPI} 1$

CENPF,HLAG,HMOX1,TTK,S 100A6,S100A10,S 100A11,HP,VEGF A,ANXA1,GDF15 ,PRDX4,BIRC5,S TC2,GAPDH,GBP 1,GBP2,BNIP3L,B ST2,PYGL,PAPSS 1,ECT2,TFAP2A, LGALS1,LGALS3 ,NAMPT,LPL,TO P2A,RPL7,MAD2 L1,TPI

Biological Process(BP), Cellular Component(CC) and Molecular Functions (MF)

Table 5 The enriched GO terms of the down-regulated differentially expressed genes

\begin{tabular}{|c|c|c|c|c|c|c|c|c|}
\hline GO ID & CATEGORY & GO Name & P Value & $\begin{array}{l}\text { FDR } \\
\text { B\&H }\end{array}$ & $\begin{array}{l}\text { FDR } \\
\text { B\&Y }\end{array}$ & Bonferroni & $\begin{array}{c}\text { Gene } \\
\text { Count }\end{array}$ & Gene \\
\hline GO:0099536 & $\mathrm{BP}$ & $\begin{array}{c}\text { synaptic } \\
\text { signaling }\end{array}$ & $2.15 \mathrm{E}-54$ & $9.53 \mathrm{E}-51$ & $8.55 \mathrm{E}-50$ & $9.53 \mathrm{E}-51$ & 108 & $\begin{array}{l}\text { MAG,DLGAP2,MAP1 } \\
\text { A,TSPOAP1,ASIC2,P } \\
\text { DYN,MBP,TMOD2,A } \\
\text { DCY1,CHRM3,STX1 }\end{array}$ \\
\hline
\end{tabular}


medRxiv preprint doi: https://doi.org/10.1101/2020.12.21.20248616; this version posted December 23, 2020. The copyright holder for this preprint (which was not certified by peer review) is the author/funder, who has granted medRxiv a license to display the preprint in perpetuity. All rights reserved. No reuse allowed without permission.

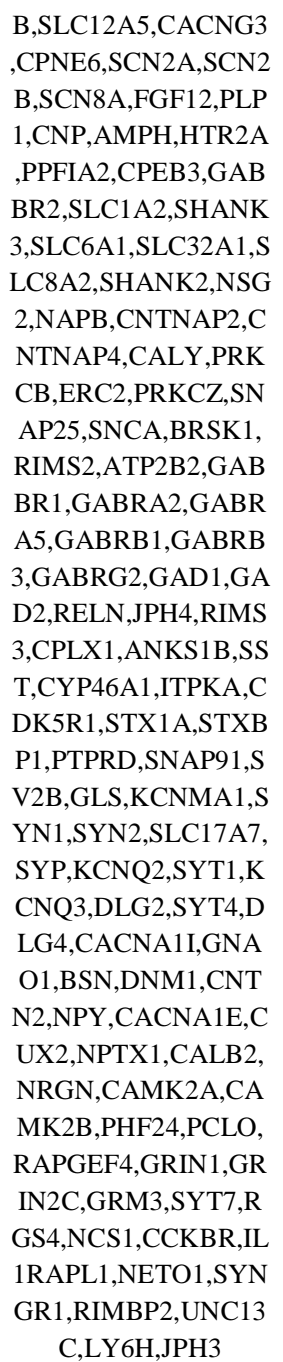

MAG,DLGAP2,MAP1

A,TSPOAP1,ASIC2,P DYN,MBP,TMOD2,C HGA,NDRG2,ADCY1 ,CHRM3,STX1B,SLC 12A5,CACNG3,CPNE 6,AMER2,SCN2A,SC N2B,SCN8A,FGF12,F GFR3,PLP1,CNP,VIP, AMPH,SFRP1,VSNL1 ,HTR2A,PPFIA2,PKP 4,CPEB3,MYRIP,GA BBR2,PEX5L,SLC1A 2,SHANK3,SLC6A1,S LC32A1,SLC8A2,AR RB1,SHANK2,NSG2, NAPB,ASIP,CNTNAP 2,CNTNAP4,CALY,P RKCB,ERC2,PRKCZ, SNAP25,SNCA,BRSK 1,RIMS2,ATP2B2,GA BBR1,SCN3B,GABR A2,GABRA5,GABRB 
medRxiv preprint doi: https://doi.org/10.1101/2020.12.21.20248616; this version posted December 23, 2020. The copyright holder for this preprint (which was not certified by peer review) is the author/funder, who has granted medRxiv a license to display the preprint in perpetuity. All rights reserved. No reuse allowed without permission.

O1,SYNGR1,DAAM2,

RIMBP2,UNC13C,LY $6 \mathrm{H}, \mathrm{JPH} 3$

EPHB6,MAG,DOK6,E RBB3,MAP1 A,MAP4, ENPP2,ADCY1,SLIT

RK1,STX1B,SLC12A

5,UNC5A,CPNE6,SE RPINI1,OLFM1,UGT8

,FGFR3,PLP1,CNP,R ASAL1,SEMA4D,SFR P1,P2RY12,PACSIN1, FA2H,SH3GL2,SH3G L3,CPEB3,APOD,SH TN1,ZNF365,SHANK 3,S1PR5,SHANK2,EF HD1,VSTM2L,CHD5, ASPA,CNTNAP2,KN DC1,DNM3,PRKCZ,S NAP25,BRSK1,RIMS 2,FAIM2,ATP2B2,MI AT,GABRA5,MYT1L, GABRB1,GABRB3,S OX10,ZNF536,RELN, KLK6,NKX62,SPOCK1,SRRM4,R AP1GAP2,BRINP1,M APK8IP3,ITPKA,KIA A0319,NEFM,NEFL,S TMN4,PRDM8,CDK5 R1,STXBP1,PTPRD,S NAP91,ARHGEF7,AB CC8,BHLHE22,ARH GAP44,KCNMA1,NA V3,SYN1,SYT1,DLG2 ,SYT4,DLG4,GNAO1, KIF5C,NNAT,MYRF, CNTN2,NPY,KIAA11 
medRxiv preprint doi: https://doi.org/10.1101/2020.12.21.20248616; this version posted December 23, 2020. The copyright holder for this preprint (which was not certified by peer review) is the author/funder, who has granted medRxiv a license to display the preprint in perpetuity. All rights reserved. No reuse allowed without permission.

GO:0030182

BP

neuron differentiati

on

\author{
07,CUX2,NPTX1,RAP \\ 1GAP,CAMK1D,CA \\ MK2A,CAMK2B,SLI \\ TRK4,RAPGEF4,STM \\ N2,GJC2,GRIN1,LHX \\ 6,OMG,OPCML,CCK, \\ NCS1,IL1RAPL1,SLC \\ 39A12,LINGO1,DAA \\ M2,MTURN
}

$\begin{array}{lllll}5.04 \mathrm{E}-26 & 2.48 \mathrm{E}-23 & 2.23 \mathrm{E}-22 & 2.24 \mathrm{E}-22 & 99\end{array}$

EPHB6,MAG,DOK6, MAP1A,MAP4,ADCY 1,SLITRK1,STX1B,S LC12A5,UNC5A,CPN E6,SERPINI1,OLFM1, UGT8,FGFR3,PLP1,C NP,RASAL1,SEMA4 D,SFRP1,PACSIN1,S H3GL2,SH3GL3,CPE B3,APOD,SHTN1,ZN F365,SHANK3,S1PR5 ,SHANK2,EFHD1,VS TM2L,CHD5,CNTNA P2,KNDC1,DNM3,PR KCZ,SNAP25,BRSK1, RIMS2,FAIM2,ATP2 B2,MIAT,GABRA5,M YT1L,GABRB1,GAB RB3,ZNF536,RELN,K LK6,NKX6-

2,SPOCK1,SRRM4,R AP1GAP2,BRINP1,M APK8IP3,ITPKA,KIA A0319,NEFM,NEFL,S TMN4,PRDM8,CDK5 R1,STXBP1,PTPRD,S NAP91,BHLHE22,AR HGAP44,KCNMA1,S YN1,SYT1,DLG2,SY T4,DLG4,GNAO1,KIF 5C,NNAT,CNTN2,NP Y,KIAA1 107,CUX2,N PTX1,RAP1GAP,CA MK1D,CAMK2A,CA MK2B,SLITRK4,RAP GEF4,STMN2,GRIN1, LHX6,OMG,OPCML, CCK,NCS1,IL1RAPL 1,SLC39A12,LINGO1, MTURN

MAG,ASIC2,ENPP2,S LITRK1,STX1B,CPN E6,SERPINI1,OLFM1, FGFR3,RASAL1,SEM A4D,SFRP1,P2RY12, PACSIN1,SH3GL3,CP EB3,LINGO2,SHTN1, ZNF365,SHANK3,S1P R5,SHANK2,ASPA,K NDC1,DNM3,SNAP2 
medRxiv preprint doi: https://doi.org/10.1101/2020.12.21.20248616; this version posted December 23, 2020. The copyright holder for this preprint (which was not certified by peer review) is the author/funder, who has granted medRxiv a license to display the preprint in perpetuity. All rights reserved. No reuse allowed without permission.

GO:0006812

GO:0050877
BP

BP cation transport

nervous

system

process
5,SNCA,BRSK1,RIM S2,SOX10,ZNF536,R ELN,KLK6,NKX62,SPOCK1,RAP1GAP 2,BRINP1,MAPK8IP3 ,ITPKA,KIAA0319,N EFM,NEFL,CDK5R1, PTPRD,SNAP91,ABC C8,ARHGAP44,SYT1, SYT4,DLG4,MYRF,C NTN2,CUX2,NPTX1, RAP1GAP,CAMK1D, CAMK2A,CAMK2B,S LITRK4,RAPGEF4,ST MN2,GJC2,GRIN1,O MG,NCS1,IL1RAPL1, SLC39A12,LINGO1,C BLN2,DAAM2

PDE2A,TSPOAP1,ASI C2,KCNIP3,SLC12A5 ,CACNG3,HPCA,SCN 2A,SCN2B,SCN8A,F GF12,HSPA2,KCNT1, SYT13,VIP,HTR2A,P 2RY12,KCNK12,PEX 5L,SLC1 A2,SHANK3, SLC6A1,SLC32A1,SL C8A2,SHANK2,PLCH 2,PRKCB,PRKCZ,SN AP25,SNCA,ATP1B1, ATP2B2,GABBR1,SC N3B,RELN,ATP6V1G 2,JPH4,SLC6A17,SLC 30A3,NKAIN2,KCNK 4,KLHL3,NEFL,STX1 $\mathrm{A}, \mathrm{KCNJ} 4, \mathrm{ABCC} 8, \mathrm{KC}$ NJ6,KCNMA1,SLC17 A7,KCNAB1,KCNQ2, SYT1,KCNQ3,KCNS1 ,DLG2,SYT4,DLG4,C ACNA1I,GNAO1,CA CNA1E,CAMK2A,CA MK2B,CAPN3,SLC6 A15,GJC2,GRIN1,GRI N2C,SYT7,TF,RGS4, RGS7,NCS1,CCKBR, KCNH3,IL1RAPL1,S LC39A12,NETO1,SL

C7A10,JPH3

MAG,DLGAP2,MAP1 A,ASIC2,MBP,NTSR2 ,TMOD2,KCNIP3,AD CY1,CHRM3,STX1B, SLC12A5,CACNG3,S CN2A,PPP1R1B,SCN 8A,FGF12,VIP,AMPH ,HTR2A,CPEB3,SHA NK3,SLC6A1,SLC8A 2,CRYM,SHANK2,RT 
medRxiv preprint doi: https://doi.org/10.1101/2020.12.21.20248616; this version posted December 23, 2020. The copyright holder for this preprint (which was not certified by peer review) is the author/funder, who has granted medRxiv a license to display the preprint in perpetuity. All rights reserved. No reuse allowed without permission.

GO:0030030 BP

cell projection organizatio

$\mathrm{n}$
P5,CNTNAP2,PRKCZ ,SNAP25,SNCA,BRS

K1,RIMS2,ATP2B2,G ABBR1,SCN3B,GAB RA2,GABRA5,GABR B1,GABRB3,GABRG 2,SOX10,RELN,JPH4, NKX6-

2,SRRM4,GJB6,BRIN P1,PAK6,KCNK4,NE FL,STX1A,ABCC8,G LS,KCNMA1,SLC17A 7,KCNAB1,KCNQ2,D LG2,SYT4,DLG4,CA CNA1I,CAMTA1,DN M1,MYRF,CNTN2,C ACNA1E,CUX2,NPT X1,NRGN,CAMK2B, PHF24,RAPGEF4,GRI N1,GRIN2C,GRM3,R GS4,CCK,CCKBR,NE TO1,LY6H,RCAN2,JP $\mathrm{H} 3$

EPHB6,MAG,DOK6, MAP1A,MAP4,ENPP 2,ADCY1,SLITRK1,S TX1B,SLC12A5,ERM N,UNC5A,TUBB4A,C PNE6,SERPINI1, OLF M1,UGT8,FGFR3,PLP 1,CNP,RASAL1,SEM A4D,SFRP1,P2RY12, PACSIN1,SH3GL2,CP EB3,APOD,SHTN1,Z NF365,SHANK3,SHA NK2,EFHD1, VSTM2L ,CNTNAP2,PPP1R16 B,KNDC1,DNM3,PR KCZ,SNAP25,BRSK1, ABLIM2,RIMS2,ATP 2B2,RELN,KLK6,SPO CK1,RAP1GAP2,MA PK8IP3,ITPKA,KIAA 0319,NEFM,NEFL,ST MN4,PRDM8,CDK5R 1,STXBP1,PTPRD,SN AP91,ARHGEF7,BHL HE22,ARHGAP44,NA V3,SYT1,SYT4,DLG4 ,GNAO1,KIF5C,CNT N2,NPY,KIAA1107,C UX2,NPTX1,RAP1GA P,CAMK1D,CAMK2 A,CAMK2B,SLITRK4 ,RAPGEF4,STMN2,G RIN1,OMG,CCK,NCS 1,IL1RAPL1,SLC39A 12,LINGO1,DMTN 
medRxiv preprint doi: https://doi.org/10.1101/2020.12.21.20248616; this version posted December 23, 2020. The copyright holder for this preprint (which was not certified by peer review) is the author/funder, who has granted medRxiv a license to display the preprint in perpetuity. All rights reserved. No reuse allowed without permission.

GO:0055085

GO:0044456

\begin{abstract}
$\mathrm{CC}$
\end{abstract}
transmembr

$$
\text { ane }
$$

transport
$1.76 \mathrm{E}-11$

$3.96 \mathrm{E}-15 \quad 3.58 \mathrm{E}-13$

$3.21 \mathrm{E}-12$

(1)
87

ERBB3,PDE2A,TME M144,ABCA2,TSPOA P1,ASIC2,KCNIP3,SL C12A5,CACNG3,HPC A,SCN2A,SCN2B,SC N8A,FGF12,SVOP,HS PA2,KCNT1,HPCAL4 ,CLCA4,HTR2A,P2R Y12,KCNK12,PEX5L, SLC1A2,SHANK3,SL C6A1,SLC32A1,SLC8 A2,SHANK2,GPIHBP 1,PLCH2,SLCO1 A2,P RKCB,PRKCZ,SNAP 25,SNCA,ATP1B1,AT P2B2,ANO3,SCN3B, GABRA2,GABRA5,G ABRB1,GABRB3,GA BRG2,RELN,ATP6V1 G2,JPH4,SLC6A17,CP LX1,SLC30A3,KCNK 4,NEFL,STX1A,SV2B ,KCNJ4,ABCC8,KCN J6,KCNMA1,SLC17A 7,KCNAB1,KCNQ2,K CNQ3,KCNS1,DLG2, DLG4,CACNA1I,NPY ,ANO4,CACNA1E,CA PN3,SLC6A15,GJC2, GRIN1,GRIN2C,GRM 3,TF,RGS4,RGS7,NC S1,KCNH3,IL1RAPL1 ,SLC39A12,NETO1,S LC7A10,DMTN,JPH3

DLGAP2,ERBB3,PDE 2A,MAP1A,MAP4,TS POAP1,ASIC2,PDYN, KCNIP3,ADCY1,CHR M3,SYNPR,SLITRK1, STX1B,CACNG3,HP CA,CAMKV,SCN2A, KCTD16,PPP1R1B,SC N8A,OLFM1,SVOP,A MPH,HTR2A,SEPTIN 4,PACSIN1,PPFIA2,P KP4,SH3GL2,SH3GL 3,CPEB3,BCAS1,MA GEE1,GABBR2,RAB6 B,SLC1A2,SHANK3, SH2D5,SLC6A1,SLC3 2A1,SLC8A2,ARRB1, SHANK2,NAPB,LGI3 ,CNTNAP2,CNTNAP 4,DNM3,CALY,PRKC B,ERC2,PRKCZ,SNA P25,SNCA,BRSK1,RI MS2,FAIM2,ATP2B2, GABBR1,GABRA2,G ABRA5,GABRB1,GA BRB3,GABRG2,GAD 
medRxiv preprint doi: https://doi.org/10.1101/2020.12.21.20248616; this version posted December 23, 2020. The copyright holder for this preprint (which was not certified by peer review) is the author/funder, who has granted medRxiv a license to display the preprint in perpetuity. All rights reserved. No reuse allowed without permission.

GO:0043005

K,NCS1,IL1RAPL1,M

AL2,INA,NETO1,SY

NGR1,RIMBP2,UNC1

3C,LY6H,EPB41L1,D MTN

EPHB6,MAG,DLGAP 2,PDE2A,MAP1A,MA P4,TSPOAP1,ASIC2,P DYN,MBP,NTSR2,T MOD2,KCNIP3,NDR G2,PLEKHB1,ADCY 1 ,CHRM3,ADCY2,SY NPR,STX1B,SLC12A 5,ERMN,CACNG3,HP CA,UNC5A,TUBB4A, CPNE6,SCN2A,PPP1 R1B,SCN8A, OLFM1, VIP,AMPH,NECAB2, HTR2A,PACSIN1,SH 3GL2,CPEB3,MYRIP, APOD,MAGEE1,GAB BR2,SHTN1,PEX5L,S LC1A2,SHANK3,SLC 6A1,SLC32A1,SLC8A 2,ARRB1,SHANK2,N SG2,VSTM2L,LGI3,C NTNAP2,KNDC1,CN TNAP4,DNM3,MAST 1,PRKCB,ERC2,PRK CZ,SNAP25,NRSN1,S NCA,BRSK1,RIMS2, ATP2B2,GABBR1,GA BRA2,GABRA5,GAB RB1,GABRB3,GABR G2,GAD1,GAD2,NM

NAT2,RELN,JPH4,KI F1A,SPOCK1,CPLX1, ANKS1B,RAP1GAP2, 
medRxiv preprint doi: https://doi.org/10.1101/2020.12.21.20248616; this version posted December 23, 2020. The copyright holder for this preprint (which was not certified by peer review) is the author/funder, who has granted medRxiv a license to display the preprint in perpetuity. All rights reserved. No reuse allowed without permission.

GO:0036477

CC

somatodend

2.01E-51

$1.94 \mathrm{E}-49$

1.35E-48

$1.16 \mathrm{E}-48$

compartme

nt

DDN,LAMP5,RGS11, BRINP1,SLC30A3,CY P46A1,MAPK8IP3,IT

PKA,NEFM,NEFL,ST MN4,CDK5R1,STX1 A,STXBP1,SEPTIN3, PTPRD,SNAP91,ARH GEF7,SV2B,KCNJ4,K CNJ6,ARHGAP44,KC NMA1,SYN1,SLC17A 7,SYP,KCNAB1,KCN Q2,SYT1,KCNQ3,DL G2,SYT4,DLG4,GNA O1,KIF5C,BSN,DNM 1,GNG3,CNTN2,DYN C1I1,NPY,KIAA1107, KLC1,NPTX1,RAP1G AP,CALB2,RGS7BP, NRGN,CAMK2A,CA MK2B,PCLO,RAPGE F4,STMN2,GJC2,GRI N1,MAP7,GRM3,SYT 7,RGS7,CCK,NCS1,T TLL7,IL1RAPL1,MA L2,UNC13C,SLC7A10

DLGAP2,PDE1A,PDE 2A,MAP1A,ASIC2,PD YN,MBP,NTSR2,KC NIP3,ADCY1,CHRM3 ,ADCY2,SLC12A5,ER MN,CACNG3,CKMT

1B,HPCA,UNC5A,TU BB4A,CPNE6,SERPI NI1,PPP1R1B,SCN8A ,OLFM1,VIP,NECAB 2,HTR2A,SH3GL2,CP EB3,APOD,MAGEE1, SHTN1,PEX5L,SLC1 A2,SHANK3,SLC32A 1,SLC8A2,ARRB1,SH ANK2,NSG2,CNTNA P2,KNDC1,CNTNAP4 ,DNM3,MAST1,ERC2 ,PRKCZ,SNAP25,NR SN1,SNCA,ATP2B2, GABBR1,GABRA2,G ABRA5,GABRB1,RE LN,JPH4,KIF1A,CPL X1,ANKS1B,DDN,RG S11,BRINP1,SST,SLC 30A3,CYP46A1,MAP K8IP3,ITPKA,NEFM, CDK5R1,PTPRD,SNA P91,ARHGEF7,KCNJ 4,KCNJ6,ARHGAP44, KCNMA1,SYN1,KCN AB1,SYT1,KCNQ3,D LG2,SYT4,DLG4,GN AO1,KIF5C,BSN,DN M1,GNG3,CNTN2,NP 
medRxiv preprint doi: https://doi.org/10.1101/2020.12.21.20248616; this version posted December 23, 2020. The copyright holder for this preprint (which was not certified by peer review) is the author/funder, who has granted medRxiv a license to display the preprint in perpetuity. All rights reserved. No reuse allowed without permission.

GO:0120038

\begin{abstract}
$\mathrm{CC}$
\end{abstract}
plasma
membrane
bounded

cell

projection

part
Y,KLC1,CACNA1E,R AP1GAP,CALB2,RGS 7BP,NRGN,CAMK2A ,CAMK2B,PCLO,RAP GEF4,STMN2,GJC2,G RIN1,GRM3,SYT7,R GS7,CCK,NCS1,TTL L7,IL1RAPL1,MAL2, SLC7A10

$3.64 \mathrm{E}-44 \quad 2.34 \mathrm{E}-42 \quad 1.62 \mathrm{E}-41 \quad 2.10 \mathrm{E}-41$ 132

MAG,DLGAP2,PDE2 A,MAP1A,MAP4,TSP OAP1,ASIC2,PDYN, MBP,NTSR2,TMOD2, KCNIP3,NDRG2,PLE KHB1,ADCY1,CHRM 3,ADCY2,SLC12A5,E RMN,CACNG3,HPCA ,UNC5A,TUBB4A,CP NE6,SCN2A,PPP1R1 $\mathrm{B}, \mathrm{SCN} 8 \mathrm{~A}, \mathrm{OLFM1,A}$ MPH,NECAB2,HTR2 A,P2RY12,SEPTIN4,P ACSIN1,SH3GL2,CPE B3,MYRIP,APOD,MA GEE1,SHTN1,PEX5L, SLC1A2,SHANK3,SL C32A1,SLC8A2,ARR B1,SHANK2,NSG2,C NTNAP2,KNDC1,CN TNAP4,DNM3,PRKC B,ERC2,PRKCZ,SNA P25,NRSN1,SNCA,B RSK1,ATP2B2,GABB R1,GABRA2,GABRA 5,GABRB1,GABRB3, GABRG2,GAD1,REL N,JPH4,KIF1 A,SPOC K1,CPLX1,ANKS1B, DDN,LAMP5,RGS11, BRINP1,SLC30A3,CY P46A1,MAPK8IP3,IT PKA,NEFL,STMN4,C DK5R1,PTPRD,SNAP 91,ARHGEF7,KCNJ4, KCNJ6,ARHGAP44,K CNMA1,SYN1,SYP,K CNAB1,KCNQ2,SYT 1,KCNQ3,DLG2,SYT 4,DLG4,GNAO1,KIF5 C,BSN,DNM1,GNG3, CNTN2,DYNC1I1,NP Y,KIAA1 107,KLC1,R AP1GAP,CALB2,RGS 7BP,NRGN,CAMK2A ,CAMK2B,PCLO,RAP GEF4,STMN2,GJC2,G RIN1,GRM3,SYT7,R GS7,CCK,NCS1,TTL L7,IL1RAPL1,MAL2, UNC13C,SLC7A10,D 
medRxiv preprint doi: https://doi.org/10.1101/2020.12.21.20248616; this version posted December 23, 2020. The copyright holder for this preprint (which was not certified by peer review) is the author/funder, who has granted medRxiv a license to display the preprint in perpetuity. All rights reserved. No reuse allowed without permission.

MTN

GO:0030425

$\mathrm{CC}$

dendrite

$1.08 \mathrm{E}-41 \quad 5.21 \mathrm{E}-40$

3.62E-39

$6.26 \mathrm{E}-39$

87

DLGAP2,PDE2A,MA P1A,ASIC2,PDYN,NT SR2,KCNIP3,ADCY1, CHRM3,ADCY2,SLC 12A5,CACNG3,HPCA ,CPNE6,PPP1R1B,SC N8A,NECAB2,HTR2

A,SH3GL2,CPEB3,AP OD,MAGEE1,PEX5L, SLC1A2,SHANK3,SL C32A1,SLC8A2,ARR B1,SHANK2,NSG2,C NTNAP2,KNDC1,CN TNAP4,DNM3,ATP2 B2,GABBR1,GABRA 2,GABRA5,GABRB1, RELN,JPH4,KIF1A,C PLX1,ANKS1B,DDN, RGS11,BRINP1,SLC3 0A3,CYP46A1,MAPK 8IP3,ITPKA,CDK5R1, PTPRD, KCNJ4,KCNJ 6,ARHGAP44,KCNM A1,SYN1,KCNAB1,S YT1,KCNQ3,DLG2,S YT4,DLG4,GNAO1,K IF5C,BSN,DNM1,GN G3,RAP1GAP,CALB2 ,RGS7BP,NRGN,CA MK2A,CAMK2B,PCL O,RAPGEF4,GRIN1, GRM3,SYT7,RGS7,C CK,NCS1,TTLL7,IL1 RAPL1,MAL2,SLC7A

10

GO:0044297

$\mathrm{CC}$

cell body

2.39E-32 9.19E-31

6.38E-30

$1.38 \mathrm{E}-29$

78

PDE1A,MAP1A,ASIC 2,PDYN,MBP,NTSR2, SLC12A5,ERMN,CK MT1B,HPCA,UNC5A, TUBB4A,CPNE6,SER PINI1,PPP1R1B,SCN8 A,OLFM1,VIP,HTR2 A,P2RY12,SH3GL2,A POD,SHTN1,SLC8A2, SHANK2,CNTNAP2, KNDC1,MAST1,ERC 2,PRKCZ,SNAP25,NR SN1,SNCA,ATP2B2, GABBR1,GABRA2,G ABRA5,RELN,KIF1A ,CPLX1,DDN,BRINP1 ,SST,MAPK8IP3,NEF M,CDK5R1,SNAP91, ARHGEF7,KCNJ4,KC NJ6,KCNMA1,KCNA B1,KCNQ3,DLG2,SY T4,GNAO1,KIF5C,BS N,GNG3,CNTN2,NPY 
medRxiv preprint doi: https://doi.org/10.1101/2020.12.21.20248616; this version posted December 23, 2020. The copyright holder for this preprint (which was not certified by peer review) is the author/funder, who has granted medRxiv a license to display the preprint in perpetuity. All rights reserved. No reuse allowed without permission.

GO:0030054

$\mathrm{CC}$

cell junction

intrinsic

component

of plasma

membrane
,KIAA1107,KLC1,CA CNA1E,RAP1GAP,R GS7BP,NRGN,CAMK 2A,CAMK2B,PCLO,R APGEF4,STMN2,GJC 2,GRIN1,SYT7,CCK, TTLL7,SLC7A10

DLGAP2,CHRM3,SY NPR,SLITRK1,SCN2 A,KCTD16,OPALIN, OLFM1,FGFR3,SVOP ,AMPH,PACSIN1,PK P4,CPEB3,GABBR2,S HANK3,SH2D5,SHA NK2,STXBP6,LGI3,C NTNAP2,CNTNAP4,E RC2,PRKCZ,SNAP25, SNCA,BRSK1,ATP1B 1,RIMS2,FAIM2,ATP 2B2,GABBR1,GABR A2,GABRA5,GABRB 1,GABRB3,GABRG2, GAD2,SLC6A17,GJB 6,RIMS3,ANKS1B,LA MP5,PAK6,SLC30A3, CYP46A1,STX1A,SE PTIN3,ARHGEF7,SV 2B,KCNJ4,ARHGAP4 4,SYN1,SYN2,SLC17 A7,SYP,SYT1,DLG2, SYT4,DLG4,BSN,KIA A1107,CALB2,NRGN ,CAMK2A,CAMK2B, PCLO,GJC2,GRIN1,G RIN2C,SYT7,CLDN1 0,NCS1,NETO1,SYN GR1,RIMBP2,UNC13 $\mathrm{C}, \mathrm{CDH} 18$

$\begin{array}{lllll}2.36 \mathrm{E}-12 & 2.53 \mathrm{E}-11 & 1.75 \mathrm{E}-10 & 1.37 \mathrm{E}-09 & 79\end{array}$

EPHB6,MAG,ERBB3, ASIC2,ENPP2,NTSR2 ,KCNIP3,ADCY1,CH RM3,ADCY2,SLITRK 1,SLC12A5,CACNG3, UNC5A,SCN2A,SCN2 B,SCN8A,OLFM1,FG FR3,SYT13, VIPR1,SE MA4D,CLCA4,HTR2 A,P2RY12,KCNK12, GABBR2,SLC1A2,SH ANK3,SLC6A1,SLC3 2A1,SLC8A2,SHANK 2,GPIHBP1,SLCO1A2 ,CNTNAP2,CNTNAP 4,CALY,SNAP25,ATP 1B1,ATP2B2,GABBR 1,SCN3B,GABRA2,G ABRA5,GABRB1,GA BRB3,GABRG2,SLC6 A17,SLC30A3,KCNK 
medRxiv preprint doi: https://doi.org/10.1101/2020.12.21.20248616; this version posted December 23, 2020. The copyright holder for this preprint (which was not certified by peer review) is the author/funder, who has granted medRxiv a license to display the preprint in perpetuity. All rights reserved. No reuse allowed without permission.

GO:0098805

GO:0099503
CC

membrane

\author{
4,TMEM130,STX1A,P \\ TPRD,KCNJ4,ABCC8 \\ ,KCNJ6,KCNMA1,KC \\ NAB1,KCNQ2,KCNQ \\ 3,KCNS1,DLG2,DLG \\ 4,CNTN2,CACNA1E, \\ RGS7BP,GPR37,SLC6 \\ A15,GRIN1,GRIN2C, \\ GRM3,SYT7,PTPRT, \\ KCNH3,IL1RAPL1,S \\ LC39A12,NETO1,SL \\ C7A10
}

4.80E-09 3.80E-08

2.63E-07

2.77E-06

70

MAG,PDE2A,ABCA2 ,MAL,CHGA,ADCY1, ADCY2,SYNPR,CAC NG3,UNC5A,SVOP,C NP,MOG,PARM1,AM PH,HTR2A,P2RY12,S EPTIN4,SH3GL2,SH3 GL3,MYRIP,PEX5L,S LC32A1,ARRB1,NSG 2,NAPB,PRKCZ,SNA P25,RAB11FIP4,SNC A,ATP1B1,FAIM2,AT P2B2,GABBR1,GABR A2,SOX10,GAD1,GA D2,ATP6V1G2,SLC6 A17,LAMP5,SLC30A 3,KIAA0319,STX1A,S NAP91,SV2B,GPR62, ABCC8,KCNMA1,NA V3,SYN1,SYN2,SLC1 7A7,SYP,SYT1,KCN Q3,SYT4,DLG4,BSN, KIAA1107,NRGN,CA MK2A,CAMK2B,SYT 7,TF,RGS7,MAL2,PL LP,SYNGR1,UNC13C

PDYN,CHGA,CHGB, SYNPR,STX1B,SERP INI1,SVOP,SYT13,A MPH,SEPTIN4,SH3G L2,SH3GL3,MYRIP,S LC32A1,LGI3,SNAP2 5,SNCA,BRSK1,GAB BR1,GABRA2,GAD2, KLK7,KLK6,ATP6V1 G2,KIF1A,SLC6A17, LAMP5,SLC30A3,ST X1A,STXBP1,SNAP9 1,SV2B,ABCC8,SYN1 ,SYN2,SLC17A7,SYP, SYT1,SYT4,DLG4,BS N,DNM1,KIAA1107, GRIN1,SYT7,TF,NCS 1,MAL2,SYNGR1,UN C13C 
medRxiv preprint doi: https://doi.org/10.1101/2020.12.21.20248616; this version posted December 23, 2020. The copyright holder for this preprint (which was not certified by peer review) is the author/funder, who has granted medRxiv a license to display the preprint in perpetuity. All rights reserved. No reuse allowed without permission.

\begin{tabular}{|c|c|c|c|c|c|c|c|c|}
\hline GO:0098796 & $\mathrm{CC}$ & $\begin{array}{c}\text { membrane } \\
\text { protein } \\
\text { complex }\end{array}$ & $6.69 \mathrm{E}-08$ & $4.90 \mathrm{E}-07$ & $3.40 \mathrm{E}-06$ & $3.87 \mathrm{E}-05$ & 55 & $\begin{array}{c}\text { ABCA2,KCNIP3,STX } \\
\text { 1B,CACNG3,SCN2A, } \\
\text { SCN2B,SCN8A,OLF } \\
\text { M1,HSPA2,GABBR2, } \\
\text { PEX5L,SHANK3,SHA } \\
\text { NK2,NAPB,CNTNAP } \\
\text { 2,DNM3,CALY,SNAP } \\
\text { 25,ATP1B1,GABBR1, } \\
\text { SCN3B,GABRA2,GA } \\
\text { BRA5,GABRB1,GAB } \\
\text { RB3,GABRG2,ATP6V } \\
\text { 1G2,GJB6,CPLX1,KC } \\
\text { NK4,STX1A,SNAP91, } \\
\text { KCNJ4,ABCC8,KCNJ } \\
\text { 6,KCNMA1,SYN2,KC } \\
\text { NAB1,KCNQ2,SYT1, } \\
\text { KCNQ3,KCNS1,DLG } \\
\text { 2,DLG4,CACNA1I,G } \\
\text { NAO1,DNM1,GNG3, } \\
\text { KIAA1107,CACNA1E } \\
\text {,GJC2,GRIN1,GRIN2 } \\
\text { C,TF,CDH18 }\end{array}$ \\
\hline GO:0022839 & MF & $\begin{array}{c}\text { ion gated } \\
\text { channel } \\
\text { activity }\end{array}$ & $2.83 \mathrm{E}-19$ & $2.76 \mathrm{E}-16$ & $2.06 \mathrm{E}-15$ & $2.76 \mathrm{E}-16$ & 47 & $\begin{array}{c}\text { TSPOAP1,ASIC2,KC } \\
\text { NIP3,CACNG3,HPCA } \\
\text {,SCN2A,SCN2B,SCN } \\
\text { 8A,KCNT1,CLCA4,K } \\
\text { CNK12,PEX5L,SHAN } \\
\text { K3,SHANK2,SNAP25 } \\
\text {,ANO3,SCN3B,GABR } \\
\text { A2,GABRA5,GABRB } \\
\text { 1,GABRB3,GABRG2, } \\
\text { RELN,JPH4,KCNK4, } \\
\text { NEFL,KCNJ4,ABCC8 } \\
\text {,KCNJ6,KCNMA1,SL } \\
\text { C17A7,KCNAB1,KC } \\
\text { NQ2,KCNQ3,KCNS1, } \\
\text { DLG2,DLG4,CACNA } \\
\text { 1I,ANO4,CACNA1E, } \\
\text { GRIN1,GRIN2C,NCS } \\
\text { 1,KCNH3,IL1RAPL1, } \\
\text { NETO1,JPH3 }\end{array}$ \\
\hline GO:0015267 & MF & $\begin{array}{l}\text { channel } \\
\text { activity }\end{array}$ & $1.80 \mathrm{E}-18$ & 4.69E-16 & $3.50 \mathrm{E}-15$ & $1.76 \mathrm{E}-15$ & 56 & $\begin{array}{c}\text { PDE2A,TSPOAP1,ASI } \\
\text { C2,KCNIP3,CACNG3, } \\
\text { HPCA,SCN2A,SCN2B } \\
\text {,SCN8A,FGF12,KCN } \\
\text { T1,HPCAL4,CLCA4, } \\
\text { KCNK12,PEX5L,SHA } \\
\text { NK3,SHANK2,PRKC } \\
\text { B,PRKCZ,SNAP25,A } \\
\text { NO3,SCN3B,GABRA } \\
\text { 2,GABRA5,GABRB1, } \\
\text { GABRB3,GABRG2,R } \\
\text { ELN,JPH4,KCNK4,N } \\
\text { EFL,STX1A,KCNJ4,A } \\
\text { BCC8,KCNJ6,KCNM } \\
\text { A1,SLC17A7,KCNAB } \\
\text { 1,KCNQ2,KCNQ3,KC } \\
\text { NS1,DLG2,DLG4,CA } \\
\text { CNA1I,NPY,ANO4,C }\end{array}$ \\
\hline
\end{tabular}


medRxiv preprint doi: https://doi.org/10.1101/2020.12.21.20248616; this version posted December 23, 2020. The copyright holder for this preprint (which was not certified by peer review) is the author/funder, who has granted medRxiv a license to display the preprint in perpetuity. All rights reserved. No reuse allowed without permission.

GO:0005215

GO:0015318
MF

\author{
transporter \\ activity
}

$1.08 \mathrm{E}-16$

MF

$$
\begin{aligned}
& \text { molecular } \\
& \text { entity } \\
& \text { transmembr }
\end{aligned}
$$

ane

transporter activity
ACNA1E,GJC2,GRIN 1,GRIN2C,GRM3,NC S1,KCNH3,IL1RAPL1 ,NETO1,JPH3

PDE2A,TMEM144,A BCA2,TSPOAP1,ASI C2,KCNIP3,SLC12A5 ,CACNG3,HPCA,CPN E6,SCN2A,SCN2B,SC N8A,FGF12,SVOP,HS PA2,KCNT1,HPCAL4 ,CLCA4,KCNK12,AP OD,PEX5L,SLC1 A2,S HANK3,SLC6A1,SLC 32A1,SLC8A2,SHAN K2,GPIHBP1,SLCO1 A2,PRKCB,PRKCZ,S NAP25,SNCA,ATP1B 1,ATP2B2,ANO3,SCN 3B,GABRA2,GABRA 5,GABRB1,GABRB3, GABRG2,RELN,ATP 6V1G2,JPH4,SLC6A1 7,CPLX1,SLC30A3,K CNK4,NEFL,STX1A, SV2B,KCNJ4,ABCC8, KCNJ6,KCNMA1,SY N1,SLC17A7,KCNAB 1,KCNQ2,KCNQ3,KC NS1,DLG2,DLG4,CA CNA1I,NPY,ANO4,C ACNA1E,SLC6A15,G JC2,GRIN1,GRIN2C, GRM3,TF,NCS1,KCN H3,IL1RAPL1,SLC39 A12,NETO1,SLC7A10 ,JPH3

$\begin{array}{llll}3.25 \mathrm{E}-16 & 3.97 \mathrm{E}-14 & 2.96 \mathrm{E}-13 \quad 3.18 \mathrm{E}-13 \quad 67 & \end{array}$

PDE2A,TSPOAP1,ASI C2,KCNIP3,SLC12A5 ,CACNG3,HPCA,SCN 2A,SCN2B,SCN8A,F GF12,HSPA2,KCNT1, CLCA4,KCNK12,PEX 5L,SLC1A2,SHANK3, SLC6A1,SLC32A1,SL C8A2,SHANK2,SLCO 1A2,PRKCZ,SNAP25, ATP1B1,ATP2B2,AN O3,SCN3B,GABRA2, GABRA5,GABRB1,G ABRB3,GABRG2,RE LN,ATP6V1G2,JPH4, SLC6A17,SLC30A3,K CNK4,NEFL,STX1A, $\mathrm{KCNJ} 4, \mathrm{ABCC} 8, \mathrm{KCNJ}$ 6,KCNMA1,SLC17A7 ,KCNAB1,KCNQ2,K CNQ3,KCNS1,DLG2, DLG4,CACNA1I,AN 
medRxiv preprint doi: https://doi.org/10.1101/2020.12.21.20248616; this version posted December 23, 2020. The copyright holder for this preprint (which was not certified by peer review) is the author/funder, who has granted medRxiv a license to display the preprint in perpetuity. All rights reserved. No reuse allowed without permission.

\begin{tabular}{|c|c|c|c|c|c|c|c|c|}
\hline GO:0005261 & $\mathrm{MF}$ & $\begin{array}{l}\text { cation } \\
\text { channel } \\
\text { activity }\end{array}$ & $4.53 \mathrm{E}-14$ & $3.16 \mathrm{E}-12$ & $2.36 \mathrm{E}-11$ & $4.42 \mathrm{E}-11$ & 42 & $\begin{array}{c}\text { PDE2A,TSPOAP1,ASI } \\
\text { C2,KCNIP3,CACNG3, } \\
\text { HPCA,SCN2A,SCN2B } \\
\text {,SCN8A,FGF12,KCN } \\
\text { T1,KCNK12,PEX5L,S } \\
\text { HANK3,SHANK2,PR } \\
\text { KCZ,SNAP25,SCN3B, } \\
\text { RELN,JPH4,KCNK4, } \\
\text { NEFL,STX1A,KCNJ4, } \\
\text { ABCC8,KCNJ6,KCN } \\
\text { MA1,KCNAB1,KCNQ } \\
\text { 2,KCNQ3,KCNS1,DL } \\
\text { G2,DLG4,CACNA1, } \\
\text { CACNA1E,GRIN1,GR } \\
\text { IN2C,NCS1,KCNH3,I } \\
\text { L1RAPL1,NETO1,JP } \\
\text { H3 }\end{array}$ \\
\hline GO:0005244 & $\mathrm{MF}$ & $\begin{array}{l}\text { voltage- } \\
\text { gated ion } \\
\text { channel } \\
\text { activity }\end{array}$ & $1.74 \mathrm{E}-13$ & $1.13 \mathrm{E}-11$ & $8.46 \mathrm{E}-11$ & $1.70 \mathrm{E}-10$ & 31 & $\begin{array}{c}\text { TSPOAP1,KCNIP3,C } \\
\text { ACNG3,HPCA,SCN2 } \\
\text { A,SCN2B,SCN8A,KC } \\
\text { NT1,KCNK12,SNAP2 } \\
\text { 5,SCN3B,RELN,KCN } \\
\text { K4,NEFL,KCNJ4,AB } \\
\text { CC8,KCNJ6,KCNMA } \\
\text { 1,KCNAB1,KCNQ2,K } \\
\text { CNQ3,KCNS1,DLG2, } \\
\text { DLG4,CACNA1I,CAC } \\
\text { NA1E,GRIN1,GRIN2 } \\
\text { C,NCS1,KCNH3,IL1R } \\
\text { APL1 }\end{array}$ \\
\hline GO:0015077 & $\mathrm{MF}$ & $\begin{array}{l}\text { monovalent } \\
\text { inorganic } \\
\text { cationtrans } \\
\text { membrane } \\
\text { transporter } \\
\text { activity }\end{array}$ & $1.63 \mathrm{E}-08$ & $6.11 \mathrm{E}-07$ & $4.56 \mathrm{E}-06$ & $1.59 \mathrm{E}-05$ & 32 & $\begin{array}{c}\text { ASIC2,KCNIP3,SLC1 } \\
\text { 2A5,SCN2A,SCN2B,S } \\
\text { CN8A,FGF12,KCNT1, } \\
\text { KCNK12,SLC1A2,SL } \\
\text { C6A1,SLC32A1,SLC8 } \\
\text { A2,PRKCZ,SNAP25, } \\
\text { ATP1B1,SCN3B,ATP } \\
\text { 6V1G2,SLC6A17,KC } \\
\text { NK4,KCNJ4,ABCC8, } \\
\text { KCNJ6,KCNMA1,SL } \\
\text { C17A7,KCNAB1,KC } \\
\text { NQ2,KCNQ3,KCNS1, } \\
\text { SLC6A15,KCNH3,NE } \\
\text { TO1 }\end{array}$ \\
\hline GO:0008092 & $\mathrm{MF}$ & $\begin{array}{l}\text { cytoskeletal } \\
\text { protein } \\
\text { binding }\end{array}$ & $1.86 \mathrm{E}-07$ & $5.49 \mathrm{E}-06$ & 4.10E-05 & $1.81 \mathrm{E}-04$ & 48 & $\begin{array}{r}\text { MAP1A,MAP4,MAP6 } \\
\text { D1,TMOD2,ERMN,H } \\
\text { PCA,HSPA2,MOBP,P } \\
\text { ACSIN1,MYRIP,JAK } \\
\text { MIP3,SHTN1,RAB6B, } \\
\text { SHANK3,TAGLN3,P } \\
\text { HACTR3,DNM3,MAS } \\
\text { T1,SNAP25,SNCA,BR }\end{array}$ \\
\hline
\end{tabular}


medRxiv preprint doi: https://doi.org/10.1101/2020.12.21.20248616; this version posted December 23, 2020. The copyright holder for this preprint (which was not certified by peer review) is the author/funder, who has granted medRxiv a license to display the preprint in perpetuity. All rights reserved. No reuse allowed without permission.

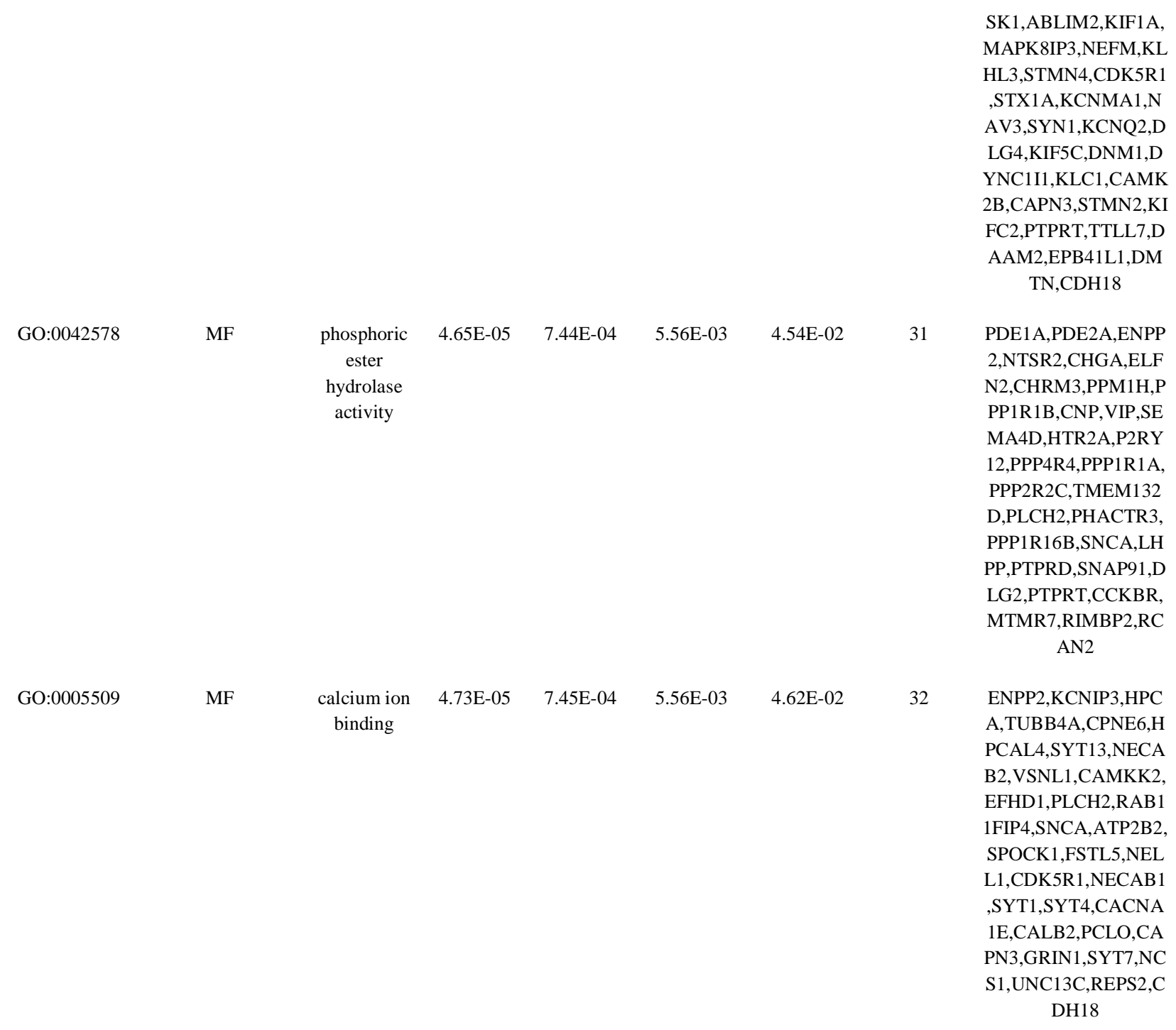

Biological Process(BP), Cellular Component(CC) and Molecular Functions (MF)

Table 6 Topology table for up and down regulated genes

\begin{tabular}{ccccccc} 
Regulation & Node & Degree & Betweenness & Stress & Closeness & $\begin{array}{c}\text { Clustering } \\
\text { Coefficient }\end{array}$ \\
\hline Up & MYC & 466 & 0.190681 & 51844356 & 0.372325 & 0.001396 \\
Up & VCAM1 & 407 & 0.167534 & 39964488 & 0.38313 & 0.010219 \\
Up & CDK2 & 255 & 0.082306 & 21529624 & 0.346908 & 0.005741 \\
Up & HNRNPA1 & 194 & 0.056605 & 11406324 & 0.346147 & 0.008126 \\
Up & PCNA & 163 & 0.047423 & 8562626 & 0.33664 & 0.006677 \\
Up & EEF1A1 & 160 & 0.0507 & 9598468 & 0.343541 & 0.016206
\end{tabular}


medRxiv preprint doi: https://doi.org/10.1101/2020.12.21.20248616; this version posted December 23, 2020. The copyright holder for this preprint (which was not certified by peer review) is the author/funder, who has granted medRxiv a license to display the preprint in perpetuity. All rights reserved. No reuse allowed without permission.

\begin{tabular}{|c|c|c|c|c|c|c|}
\hline Up & VIM & 157 & 0.056692 & 8223668 & 0.341541 & 0.002262 \\
\hline Up & AURKA & 141 & 0.048499 & 9350878 & 0.326002 & 0.001877 \\
\hline Up & CDK1 & 138 & 0.058338 & 8494824 & 0.366411 & 0.018845 \\
\hline Up & RPS8 & 137 & 0.016203 & 3357782 & 0.330197 & 0.129025 \\
\hline Up & RPS2 & 130 & 0.021628 & 3899186 & 0.330836 & 0.133333 \\
\hline Up & RPL14 & 124 & 0.022345 & 3352090 & 0.328244 & 0.123394 \\
\hline Up & RPL6 & 112 & 0.010988 & 2932384 & 0.319037 & 0.154762 \\
\hline Up & HIF1A & 110 & 0.034936 & 8860554 & 0.320756 & 0.002077 \\
\hline Up & HNRNPK & 105 & 0.032023 & 5274386 & 0.348114 & 0.011803 \\
\hline Up & RPS3A & 105 & 0.013889 & 2957694 & 0.338611 & 0.213919 \\
\hline Up & RPS3 & 105 & 0.012012 & 2806866 & 0.339788 & 0.207143 \\
\hline Up & LGALS3 & 102 & 0.0419 & 6922432 & 0.300586 & 0.00202 \\
\hline Up & HSPA1A & 98 & 0.02913 & 4799060 & 0.345042 & 0.006522 \\
\hline Up & KIF11 & 94 & 0.026283 & 3693580 & 0.314998 & 0.003106 \\
\hline Up & PPP2CB & 93 & 0.025911 & 6041756 & 0.282666 & 0.004675 \\
\hline Up & TUBA1C & 92 & 0.026442 & 5469244 & 0.324226 & 0.006211 \\
\hline Up & RPS4X & 89 & 0.004167 & 1298256 & 0.330224 & 0.289326 \\
\hline Up & CAV1 & 89 & 0.028641 & 6173224 & 0.310473 & $2.55 \mathrm{E}-04$ \\
\hline Up & HSPA4 & 87 & 0.023721 & 5441308 & 0.317974 & 0.00294 \\
\hline Up & RPL18A & 83 & 0.006185 & 1583540 & 0.315313 & 0.245959 \\
\hline Up & G3BP1 & 82 & 0.023172 & 2982694 & 0.325821 & 0.006962 \\
\hline Up & HSPD1 & 82 & 0.033759 & 4662050 & 0.352023 & 0.009033 \\
\hline Up & RPL23 & 82 & 0.009988 & 1859704 & 0.326521 & 0.269497 \\
\hline Up & RPL7 & 80 & 0.003846 & 1240590 & 0.328113 & 0.310759 \\
\hline Up & YY1 & 79 & 0.026012 & 4406736 & 0.324226 & 0.008886 \\
\hline Up & RPS13 & 79 & 0.00364 & 1001966 & 0.324765 & 0.346641 \\
\hline Up & RPL7A & 78 & 0.004083 & 1215840 & 0.329086 & 0.322344 \\
\hline Up & RPS11 & 78 & 0.005768 & 1272406 & 0.329086 & 0.296037 \\
\hline Up & CEP55 & 76 & 0.0224 & 4279650 & 0.293579 & 0 \\
\hline Up & CCNB1 & 76 & 0.017352 & 3526120 & 0.32564 & 0.026667 \\
\hline
\end{tabular}


medRxiv preprint doi: https://doi.org/10.1101/2020.12.21.20248616; this version posted December 23, 2020. The copyright holder for this preprint (which was not certified by peer review) is the author/funder, who has granted medRxiv a license to display the preprint in perpetuity. All rights reserved. No reuse allowed without permission.

\begin{tabular}{|c|c|c|c|c|c|c|}
\hline Up & RPS15A & 75 & 0.002732 & 698466 & 0.317974 & 0.350991 \\
\hline Up & RPL23A & 75 & 0.004075 & 1083514 & 0.316992 & 0.310631 \\
\hline Up & CCNA2 & 74 & 0.012837 & 2401830 & 0.315773 & 0.0311 \\
\hline Up & GAPDH & 74 & 0.023578 & 4175664 & 0.341115 & 0.021518 \\
\hline Up & RPL31 & 70 & 0.006582 & 1558268 & 0.320731 & 0.315942 \\
\hline Up & KPNA2 & 68 & 0.021359 & 2605456 & 0.338807 & 0.01352 \\
\hline Up & HNRNPC & 67 & 0.014471 & 2415210 & 0.337055 & 0.024519 \\
\hline Up & ССТ8 & 66 & 0.020139 & 2977710 & 0.333794 & 0.029371 \\
\hline Up & RPS25 & 66 & 0.002959 & 753210 & 0.323945 & 0.369697 \\
\hline Up & RPS26 & 66 & 0.002063 & 824002 & 0.318616 & 0.417249 \\
\hline Up & RPL15 & 66 & 0.002164 & 790104 & 0.316919 & 0.401399 \\
\hline Up & RPS7 & 65 & 0.004992 & 1173908 & 0.331423 & 0.371635 \\
\hline Up & RAN & 61 & 0.01418 & 2141386 & 0.315482 & 0.009351 \\
\hline Up & AURKB & 57 & 0.013872 & 2386506 & 0.310285 & 0.011448 \\
\hline Up & RPLP1 & 56 & 0.007333 & 1548306 & 0.309653 & 0.198463 \\
\hline Up & SUMO2 & 55 & 0.013262 & 2231328 & 0.293286 & 0.002177 \\
\hline Up & RPS12 & 54 & $7.44 \mathrm{E}-04$ & 314992 & 0.309747 & 0.510832 \\
\hline Up & RPS10 & 53 & 0.004576 & 1037816 & 0.327694 & 0.324383 \\
\hline Up & CENPA & 47 & 0.010717 & 2251206 & 0.293516 & 0.009251 \\
\hline Up & MAD2L1 & 47 & 0.010109 & 1979706 & 0.291847 & 0.00101 \\
\hline Up & NES & 47 & 0.012095 & 1731788 & 0.306167 & 0.00555 \\
\hline Up & A2M & 46 & 0.013104 & 2291336 & 0.286372 & 0 \\
\hline Up & TOP2A & 46 & 0.010039 & 1964374 & 0.336806 & 0.03285 \\
\hline Up & ANXA2 & 45 & 0.012243 & 2456228 & 0.331852 & 0.018826 \\
\hline Up & PPIA & 43 & 0.014538 & 1675700 & 0.332523 & 0.013289 \\
\hline Up & CDCA5 & 41 & 0.008587 & 1808362 & 0.27717 & 0 \\
\hline Up & CNBP & 39 & 0.007453 & 1070522 & 0.310308 & 0.039136 \\
\hline Up & BUB1 & 39 & 0.009354 & 1147502 & 0.299817 & 0.003003 \\
\hline Up & S100A4 & 39 & 0.009325 & 1278456 & 0.309443 & 0.010511 \\
\hline Up & TाK & 38 & 0.006639 & 1296828 & 0.297321 & 0.001587 \\
\hline
\end{tabular}


medRxiv preprint doi: https://doi.org/10.1101/2020.12.21.20248616; this version posted December 23, 2020. The copyright holder for this preprint (which was not certified by peer review) is the author/funder, who has granted medRxiv a license to display the preprint in perpetuity. All rights reserved. No reuse allowed without permission.

\begin{tabular}{|c|c|c|c|c|c|c|}
\hline Up & OIP5 & 37 & 0.01105 & 1701996 & 0.271363 & 0.005042 \\
\hline Up & CD44 & 37 & 0.007072 & 1617142 & 0.295843 & 0.003361 \\
\hline Up & ETS1 & 37 & 0.006889 & 1719066 & 0.294253 & 0 \\
\hline Up & PTGES3 & 37 & 0.007373 & 2116432 & 0.289461 & 0 \\
\hline Up & TSC22D1 & 36 & 0.009666 & 929230 & 0.30052 & 0.005348 \\
\hline Up & SAP30 & 35 & 0.010164 & 1310506 & 0.268542 & 0.003361 \\
\hline Up & ANXA1 & 34 & 0.009726 & 1556076 & 0.322115 & 0.00713 \\
\hline Up & RPS18 & 34 & 8.51E-04 & 381780 & 0.320431 & 0.449198 \\
\hline Up & USP8 & 34 & 0.007424 & 1395528 & 0.288505 & 0 \\
\hline Up & LGALS1 & 32 & 0.013705 & 1888258 & 0.325176 & 0.022177 \\
\hline Up & PRSS23 & 31 & 0.007977 & 725388 & 0.296035 & 0.002151 \\
\hline Up & EIF4E & 31 & 0.006522 & 2247866 & 0.272625 & 0 \\
\hline Up & RPS28 & 31 & 0.003463 & 655088 & 0.309957 & 0.227957 \\
\hline Up & RAP1B & 31 & 0.009439 & 902256 & 0.307796 & 0.002151 \\
\hline Up & RPS27 & 31 & 0.003498 & 553940 & 0.313555 & 0.286022 \\
\hline Up & ACTR3 & 31 & 0.006035 & 1599372 & 0.283133 & 0 \\
\hline Up & TFAP2A & 30 & 0.005011 & 711102 & 0.31291 & 0.03908 \\
\hline Up & NEDD9 & 30 & 0.007142 & 1363776 & 0.281252 & 0.002646 \\
\hline Up & KIF20A & 29 & 0.008437 & 890172 & 0.279834 & 0.004926 \\
\hline Up & PTTG1 & 29 & 0.004477 & 862138 & 0.290095 & 0.002849 \\
\hline Up & UHRF1 & 29 & 0.006396 & 1099220 & 0.300015 & 0.02849 \\
\hline Up & ASPM & 28 & 0.008053 & 1331812 & 0.254352 & 0 \\
\hline Up & CLIC1 & 27 & 0.006564 & 846884 & 0.307151 & 0.005698 \\
\hline Up & GMNN & 27 & 0.006522 & 956548 & 0.294823 & 0.059829 \\
\hline Up & HMGB1 & 27 & 0.008424 & 942446 & 0.312648 & 0.02849 \\
\hline Up & ID2 & 27 & 0.006212 & 895872 & 0.282568 & 0.01 \\
\hline Up & CASP4 & 26 & 0.006228 & 799820 & 0.291951 & 0.015385 \\
\hline Up & SNRPG & 26 & 0.003876 & 1002362 & 0.258369 & 0 \\
\hline Up & NSMAF & 25 & 0.005462 & 1551654 & 0.267579 & 0.023333 \\
\hline Up & BIRC5 & 24 & 0.007573 & 949434 & 0.294168 & 0.047619 \\
\hline
\end{tabular}


medRxiv preprint doi: https://doi.org/10.1101/2020.12.21.20248616; this version posted December 23, 2020. The copyright holder for this preprint (which was not certified by peer review) is the author/funder, who has granted medRxiv a license to display the preprint in perpetuity. All rights reserved. No reuse allowed without permission.

\begin{tabular}{|c|c|c|c|c|c|c|}
\hline Up & BNIP3L & 24 & 0.008733 & 1628142 & 0.254446 & 0 \\
\hline Up & P4HA1 & 24 & 0.007927 & 1608450 & 0.278808 & 0.004329 \\
\hline Up & FTH1 & 24 & 0.003712 & 691466 & 0.273587 & 0 \\
\hline Up & HMMR & 23 & 0.004417 & 465750 & 0.288403 & 0.023715 \\
\hline Up & WEE1 & 23 & 0.002715 & 535402 & 0.296934 & 0.011858 \\
\hline Up & RPS29 & 23 & 0.001876 & 490882 & 0.279891 & 0.205534 \\
\hline Up & NEK2 & 23 & 0.003127 & 653156 & 0.274612 & 0 \\
\hline Up & PIMREG & 23 & 0.005535 & 813742 & 0.275922 & 0 \\
\hline Up & RPN2 & 22 & 0.010504 & 1654990 & 0.303384 & 0.017316 \\
\hline Up & TPI1 & 22 & 0.002813 & 497590 & 0.30278 & 0.063158 \\
\hline Up & CKAP2 & 22 & 0.003334 & 910146 & 0.249438 & 0 \\
\hline Up & TPX2 & 21 & 0.00333 & 462446 & 0.293055 & 0.02381 \\
\hline Up & $|F| 30$ & 21 & 0.005796 & 949076 & 0.259594 & 0 \\
\hline Up & PBK & 21 & 0.004882 & 473246 & 0.301446 & 0.005263 \\
\hline Up & HSPE1 & 21 & 0.004446 & 553950 & 0.310473 & 0.019048 \\
\hline Up & CLK1 & 21 & 0.004151 & 819798 & 0.26208 & 0 \\
\hline Up & ECT2 & 21 & 0.004536 & 851674 & 0.275904 & 0 \\
\hline Up & CCNB2 & 20 & 0.003562 & 482346 & 0.295566 & 0.1 \\
\hline Up & CENPF & 20 & 0.005433 & 590146 & 0.302134 & 0.006536 \\
\hline Up & TPT1 & 20 & 0.00307 & 453250 & 0.310121 & 0.065359 \\
\hline Up & CDC45 & 19 & 0.003641 & 639912 & 0.272661 & 0 \\
\hline Up & $\mathrm{S} 100 \mathrm{~A} 10$ & 19 & 0.002321 & 398192 & 0.264136 & 0.036765 \\
\hline Up & VEGFA & 19 & 0.005138 & 912334 & 0.267405 & 0.014706 \\
\hline Up & COL8A1 & 19 & 0.004514 & 974484 & 0.239557 & 0 \\
\hline Up & EIF3M & 19 & 0.004109 & 1129622 & 0.262247 & 0 \\
\hline Up & FBXO5 & 19 & 0.0023 & 788540 & 0.26017 & 0 \\
\hline Up & MMP9 & 18 & 0.004865 & 706696 & 0.267911 & 0.016667 \\
\hline Up & PTMA & 18 & 0.001015 & 246442 & 0.279662 & 0 \\
\hline Up & COL1A2 & 18 & 0.002829 & 485634 & 0.241246 & 0 \\
\hline Up & MMP7 & 18 & 0.005899 & 612816 & 0.251055 & 0 \\
\hline
\end{tabular}


medRxiv preprint doi: https://doi.org/10.1101/2020.12.21.20248616; this version posted December 23, 2020. The copyright holder for this preprint (which was not certified by peer review) is the author/funder, who has granted medRxiv a license to display the preprint in perpetuity. All rights reserved. No reuse allowed without permission.

\begin{tabular}{|c|c|c|c|c|c|c|}
\hline Up & TGIF1 & 18 & 0.003648 & 604458 & 0.289828 & 0.006536 \\
\hline Up & CPVL & 18 & 0.004133 & 638528 & 0.256001 & 0 \\
\hline Up & UBE2C & 17 & 0.002218 & 704108 & 0.248022 & 0 \\
\hline Up & HAT1 & 17 & 0.002517 & 323166 & 0.274741 & 0 \\
\hline Up & HJURP & 17 & 0.002708 & 533810 & 0.268717 & 0.07619 \\
\hline Up & LAMA4 & 17 & 0.004076 & 518978 & 0.30019 & 0.014706 \\
\hline Up & SF3B6 & 17 & 0.004254 & 627376 & 0.310004 & 0 \\
\hline Up & $\mathrm{CXCL8}$ & 16 & 0.004283 & 517486 & 0.267196 & 0 \\
\hline Up & EEF1B2 & 16 & 0.002838 & 408338 & 0.309887 & 0.083333 \\
\hline Up & IGFBP3 & 16 & 0.004939 & 715906 & 0.281967 & 0.008333 \\
\hline Up & HMGB2 & 16 & 0.002397 & 311774 & 0.300586 & 0.075 \\
\hline Up & PRC1 & 15 & 0.001697 & 233590 & 0.257317 & 0 \\
\hline Up & STEAP3 & 15 & 0.003991 & 567510 & 0.230454 & 0 \\
\hline Up & COL5A1 & 15 & 0.00431 & 604054 & 0.263645 & 0.009524 \\
\hline Up & COL1A1 & 15 & 0.003085 & 496550 & 0.271471 & 0.019048 \\
\hline Up & S100A6 & 15 & 0.0016 & 209634 & 0.288019 & 0.038462 \\
\hline Up & RPS27A & 15 & 0.002177 & 358862 & 0.290711 & 0.038095 \\
\hline Up & TAGLN2 & 15 & 0.003488 & 733768 & 0.302602 & 0.019048 \\
\hline Up & GNA13 & 15 & 0.004791 & 643832 & 0.217802 & 0 \\
\hline Up & NXT2 & 15 & 0.003164 & 485382 & 0.262566 & 0 \\
\hline Up & IL1RAP & 15 & 0.004924 & 895256 & 0.233388 & 0 \\
\hline Up & CKS2 & 14 & 0.002487 & 411844 & 0.301734 & 0.186813 \\
\hline Up & SERPINA3 & 14 & 0.003718 & 465246 & 0.259365 & 0 \\
\hline Up & PYGL & 14 & 0.002331 & 440042 & 0.244711 & 0 \\
\hline Up & LAMC1 & 14 & 0.003944 & 598936 & 0.260005 & 0.021978 \\
\hline Up & TNFAIP6 & 14 & 0.005036 & 753948 & 0.258956 & 0 \\
\hline Up & RPL29 & 14 & 0.001376 & 299238 & 0.294696 & 0.098901 \\
\hline Up & IL13RA2 & 14 & 0.003555 & 758816 & 0.241359 & 0 \\
\hline Up & SMS & 14 & 0.003607 & 662386 & 0.24439 & 0 \\
\hline Up & TUBB6 & 14 & 0.002688 & 405308 & 0.282802 & 0.010989 \\
\hline
\end{tabular}


medRxiv preprint doi: https://doi.org/10.1101/2020.12.21.20248616; this version posted December 23, 2020. The copyright holder for this preprint (which was not certified by peer review) is the author/funder, who has granted medRxiv a license to display the preprint in perpetuity. All rights reserved. No reuse allowed without permission.

\begin{tabular}{|c|c|c|c|c|c|c|}
\hline Up & XPR1 & 14 & 0.002899 & 569786 & 0.257575 & 0 \\
\hline Up & PDLIM1 & 13 & 0.004409 & 554816 & 0.26093 & 0.012821 \\
\hline Up & IGF2BP3 & 13 & 0.001449 & 202304 & 0.307404 & 0.038462 \\
\hline Up & HMGN1 & 13 & 0.003297 & 495018 & 0.251609 & 0 \\
\hline Up & LPL & 13 & 0.001794 & 236304 & 0.268313 & 0 \\
\hline Up & LAMB1 & 13 & 0.004044 & 361588 & 0.26475 & 0.051282 \\
\hline Up & SLBP & 13 & 0.001914 & 207260 & 0.272011 & 0.012821 \\
\hline Up & SP3 & 13 & 0.001783 & 447218 & 0.282374 & 0 \\
\hline Up & E2F2 & 13 & 0.001729 & 584938 & 0.246148 & 0 \\
\hline Up & NCAPG & 13 & 0.002006 & 428660 & 0.261213 & 0 \\
\hline Up & SOD2 & 12 & 0.001917 & 230730 & 0.284842 & 0 \\
\hline Up & STC2 & 12 & 0.003677 & 992016 & 0.245765 & 0 \\
\hline Up & GBP2 & 12 & 0.00178 & 314096 & 0.249529 & 0.015152 \\
\hline Up & AKAP12 & 12 & 0.001795 & 339040 & 0.270292 & 0 \\
\hline Up & PRDX4 & 12 & 0.002021 & 369100 & 0.257365 & 0 \\
\hline Up & SOCS2 & 11 & 0.002602 & 557558 & 0.227064 & 0 \\
\hline Up & PLAU & 11 & 0.003309 & 679596 & 0.27648 & 0 \\
\hline Up & SERPINH1 & 11 & 0.001884 & 224326 & 0.301535 & 0.018182 \\
\hline Up & CD99 & 11 & 0.002139 & 261790 & 0.278052 & 0 \\
\hline Up & CD68 & 11 & 0.002598 & 344740 & 0.256289 & 0 \\
\hline Up & RHOQ & 11 & 0.002591 & 373824 & 0.234374 & 0 \\
\hline Up & GNG10 & 11 & 0.002597 & 328426 & 0.23526 & 0 \\
\hline Up & FSTL1 & 11 & 0.003087 & 558034 & 0.226164 & 0 \\
\hline Up & VKORC1 & 11 & $9.48 \mathrm{E}-04$ & 163658 & 0.2418 & 0 \\
\hline Up & LSM5 & 11 & 0.002395 & 394548 & 0.241118 & 0 \\
\hline Up & NUSAP1 & 11 & 0.002204 & 536874 & 0.262498 & 0 \\
\hline Up & ITGA5 & 10 & 0.002624 & 336448 & 0.261896 & 0.022222 \\
\hline Up & TIMP1 & 10 & 0.001291 & 177950 & 0.247932 & 0 \\
\hline Up & HMOX1 & 10 & 0.004283 & 1202978 & 0.225666 & 0 \\
\hline Up & $\mathrm{F} 2 \mathrm{R}$ & 10 & 0.003528 & 414270 & 0.256273 & 0.022222 \\
\hline
\end{tabular}


medRxiv preprint doi: https://doi.org/10.1101/2020.12.21.20248616; this version posted December 23, 2020. The copyright holder for this preprint (which was not certified by peer review) is the author/funder, who has granted medRxiv a license to display the preprint in perpetuity. All rights reserved. No reuse allowed without permission.

\begin{tabular}{|c|c|c|c|c|c|c|}
\hline Up & CENPK & 10 & 0.002573 & 320956 & 0.250687 & 0 \\
\hline Up & HP & 9 & 0.001654 & 303090 & 0.230739 & 0 \\
\hline Up & UQCRH & 9 & 0.002217 & 323738 & 0.262851 & 0 \\
\hline Up & HLA-G & 9 & 0.001885 & 231580 & 0.249105 & 0 \\
\hline Up & S100A3 & 9 & 0.001027 & 250716 & 0.215414 & 0 \\
\hline Up & ID3 & 9 & $5.95 \mathrm{E}-04$ & 95888 & 0.227467 & 0 \\
\hline Up & ZFP36L1 & 9 & 0.001068 & 225868 & 0.245765 & 0 \\
\hline Up & MELK & 9 & 0.002037 & 357248 & 0.251701 & 0 \\
\hline Up & CHPT1 & 9 & 0.003078 & 391438 & 0.202555 & 0 \\
\hline Up & CD93 & 9 & 0.001231 & 245406 & 0.235787 & 0 \\
\hline Up & TXNL1 & 8 & 0.001545 & 368682 & 0.246874 & 0 \\
\hline Up & SEC24D & 8 & 0.00293 & 515294 & 0.218161 & 0 \\
\hline Up & LOX & 8 & 0.002166 & 362954 & 0.226525 & 0 \\
\hline Up & PHLDA1 & 8 & 0.001053 & 132000 & 0.272516 & 0 \\
\hline Up & LYZ & 8 & 0.00195 & 344810 & 0.21091 & 0 \\
\hline Up & TMSB10 & 8 & $4.27 \mathrm{E}-04$ & 113382 & 0.230299 & 0 \\
\hline Up & GBE1 & 8 & 0.002387 & 306694 & 0.232569 & 0 \\
\hline Up & BST2 & 8 & 0.001005 & 153464 & 0.24726 & 0 \\
\hline Up & WTAP & 8 & 0.002543 & 644192 & 0.218369 & 0 \\
\hline Up & DLGAP5 & 8 & $4.64 \mathrm{E}-04$ & 96656 & 0.277996 & 0 \\
\hline Up & LARP4 & 8 & 0.001079 & 252262 & 0.271921 & 0 \\
\hline Up & GGH & 8 & 0.00165 & 290688 & 0.237946 & 0 \\
\hline Up & GDF15 & 8 & 0.001555 & 170716 & 0.209159 & 0 \\
\hline Up & SNX7 & 8 & 0.002501 & 298922 & 0.188478 & 0 \\
\hline Up & PAPSS1 & 7 & 0.001362 & 166210 & 0.267126 & 0 \\
\hline Up & CA12 & 7 & 0.001958 & 352022 & 0.234843 & 0 \\
\hline Up & S100A11 & 7 & $1.00 \mathrm{E}-04$ & 16524 & 0.272534 & 0.1 \\
\hline Up & CDKN3 & 7 & $9.74 \mathrm{E}-04$ & 124826 & 0.279111 & 0.3 \\
\hline Up & HOXC6 & 7 & $7.41 \mathrm{E}-04$ & 110272 & 0.221814 & 0 \\
\hline Up & C1S & 7 & 1 & 20 & 1 & 0 \\
\hline
\end{tabular}


medRxiv preprint doi: https://doi.org/10.1101/2020.12.21.20248616; this version posted December 23, 2020. The copyright holder for this preprint (which was not certified by peer review) is the author/funder, who has granted medRxiv a license to display the preprint in perpetuity. All rights reserved. No reuse allowed without permission.

\begin{tabular}{|c|c|c|c|c|c|c|}
\hline Up & NAMPT & 7 & 0.001468 & 191052 & 0.199485 & 0 \\
\hline Up & ID4 & 7 & $1.96 \mathrm{E}-04$ & 41492 & 0.252428 & 0 \\
\hline Up & PITX1 & 7 & 0.001578 & 250120 & 0.235638 & 0 \\
\hline Up & UAP1 & 7 & $2.61 \mathrm{E}-04$ & 49694 & 0.262616 & 0 \\
\hline Up & CSRP2 & 7 & $6.62 \mathrm{E}-04$ & 177712 & 0.253676 & 0 \\
\hline Up & CYP51A1 & 7 & 0.002122 & 334352 & 0.207437 & 0 \\
\hline Up & WLS & 7 & 0.002097 & 495456 & 0.191968 & 0 \\
\hline Up & APH1A & 7 & 0.002948 & 296034 & 0.214839 & 0 \\
\hline Up & GINS2 & 7 & 0.002441 & 470260 & 0.237012 & 0 \\
\hline Up & KDM3A & 7 & 0.001984 & 309106 & 0.191253 & 0 \\
\hline Up & CLIC4 & 7 & 0.001964 & 277020 & 0.254226 & 0 \\
\hline Up & LY96 & 7 & 0.002921 & 261282 & 0.192634 & 0 \\
\hline Up & ANGPT2 & 6 & $5.17 \mathrm{E}-04$ & 68268 & 0.238929 & 0.166667 \\
\hline Up & SLC16A3 & 6 & $5.26 \mathrm{E}-04$ & 70442 & 0.236917 & 0 \\
\hline Up & PPIC & 6 & 0.00102 & 215814 & 0.220279 & 0 \\
\hline Up & SBDS & 6 & $8.62 \mathrm{E}-04$ & 280528 & 0.221491 & 0 \\
\hline Up & PLOD2 & 5 & 0.001007 & 182674 & 0.244725 & 0 \\
\hline Up & SLN & 5 & 0.001471 & 442346 & 0.156765 & 0 \\
\hline Up & F13A1 & 5 & $9.74 \mathrm{E}-04$ & 136342 & 0.244798 & 0 \\
\hline Up & COL3A1 & 5 & $5.52 \mathrm{E}-04$ & 117802 & 0.218277 & 0 \\
\hline Up & CAST & 5 & $6.10 \mathrm{E}-04$ & 77008 & 0.247722 & 0 \\
\hline Up & AK4 & 5 & 0.001052 & 205924 & 0.244071 & 0 \\
\hline Up & GBP1 & 5 & $5.10 \mathrm{E}-04$ & 106554 & 0.230196 & 0 \\
\hline Up & KDELR2 & 5 & 0.001472 & 349262 & 0.216504 & 0 \\
\hline Up & KIFC1 & 5 & $5.11 \mathrm{E}-04$ & 160300 & 0.240412 & 0 \\
\hline Up & C1QTNF1 & 5 & $5.32 \mathrm{E}-04$ & 84796 & 0.225567 & 0 \\
\hline Up & DCAF13 & 5 & 0.001047 & 226064 & 0.235828 & 0 \\
\hline Up & ZFAND6 & 5 & $9.88 \mathrm{E}-04$ & 103420 & 0.237026 & 0 \\
\hline Up & TNFRSF12A & 5 & 0.001501 & 219096 & 0.211846 & 0 \\
\hline Up & MCUB & 5 & $6.02 \mathrm{E}-04$ & 130286 & 0.229066 & 0 \\
\hline
\end{tabular}


medRxiv preprint doi: https://doi.org/10.1101/2020.12.21.20248616; this version posted December 23, 2020. The copyright holder for this preprint (which was not certified by peer review) is the author/funder, who has granted medRxiv a license to display the preprint in perpetuity. All rights reserved. No reuse allowed without permission.

\begin{tabular}{|c|c|c|c|c|c|c|}
\hline Up & MCTS1 & 5 & 0.001116 & 191346 & 0.214469 & 0 \\
\hline Up & TGFBI & 4 & 4.62E-04 & 33642 & 0.259528 & 0.166667 \\
\hline Up & COL4A1 & 4 & $2.08 \mathrm{E}-04$ & 31748 & 0.23925 & 0 \\
\hline Up & $\mathrm{CFI}$ & 4 & 0.001009 & 206118 & 0.169824 & 0 \\
\hline Up & $\mathrm{BCHE}$ & 4 & 4.87E-04 & 61622 & 0.191395 & 0 \\
\hline Up & F3 & 4 & 0.001461 & 158988 & 0.193634 & 0 \\
\hline Up & MTHFD2 & 4 & 1 & 12 & 1 & 0 \\
\hline Up & CHI3L1 & 4 & 0.001014 & 144968 & 0.206228 & 0 \\
\hline Up & PRCP & 4 & 0.001461 & 248832 & 0.212901 & 0 \\
\hline Up & TCEAL9 & 4 & 0.001461 & 259620 & 0.242944 & 0 \\
\hline Up & CCL2O & 4 & $9.81 \mathrm{E}-04$ & 173910 & 0.201601 & 0 \\
\hline Up & SLC39A14 & 4 & $5.48 \mathrm{E}-04$ & 111272 & 0.228568 & 0 \\
\hline Up & GPX7 & 4 & 0.001011 & 129746 & 0.224225 & 0 \\
\hline Up & РOTEKP & 4 & $4.94 \mathrm{E}-04$ & 85220 & 0.222499 & 0 \\
\hline Up & PLEKHA4 & 4 & $1.62 \mathrm{E}-05$ & 7254 & 0.248142 & 0 \\
\hline Up & OSTC & 4 & 0.001796 & 614424 & 0.23008 & 0 \\
\hline Up & EIF1AY & 3 & $4.90 \mathrm{E}-04$ & 80020 & 0.223019 & 0 \\
\hline Up & DBI & 3 & $9.74 \mathrm{E}-04$ & 115758 & 0.199718 & 0 \\
\hline Up & IGFBP2 & 3 & $1.25 \mathrm{E}-04$ & 22962 & 0.24906 & 0 \\
\hline Up & TNC & 3 & $1.10 \mathrm{E}-04$ & 9656 & 0.267022 & 0.333333 \\
\hline Up & MDK & 3 & $7.74 \mathrm{E}-05$ & 12700 & 0.24637 & 0 \\
\hline Up & RPS4Y1 & 3 & $9.74 \mathrm{E}-04$ & 269150 & 0.239473 & 0 \\
\hline Up & RAB13 & 3 & $9.74 \mathrm{E}-04$ & 131878 & 0.222789 & 0 \\
\hline Up & LUM & 3 & 1 & 6 & 1 & 0 \\
\hline Up & EMP1 & 3 & 0.001257 & 174202 & 0.208712 & 0 \\
\hline Up & CCZ1 & 3 & 8.17E-04 & 127366 & 0.214929 & 0 \\
\hline Up & BTG3 & 3 & $1.36 \mathrm{E}-04$ & 18980 & 0.222995 & 0 \\
\hline Up & CENPW & 3 & $5.05 \mathrm{E}-05$ & 9970 & 0.243549 & 0 \\
\hline Up & RELL1 & 3 & $1.78 \mathrm{E}-05$ & 4086 & 0.200136 & 0 \\
\hline Up & CKAP2L & 3 & $8.54 \mathrm{E}-06$ & 3422 & 0.222306 & 0 \\
\hline
\end{tabular}


medRxiv preprint doi: https://doi.org/10.1101/2020.12.21.20248616; this version posted December 23, 2020. The copyright holder for this preprint (which was not certified by peer review) is the author/funder, who has granted medRxiv a license to display the preprint in perpetuity. All rights reserved. No reuse allowed without permission.

\begin{tabular}{|c|c|c|c|c|c|c|}
\hline Up & TFPI & 3 & $6.46 \mathrm{E}-04$ & 124894 & 0.244013 & 0 \\
\hline Up & ACTG2 & 3 & $1.57 \mathrm{E}-05$ & 4618 & 0.248713 & 0 \\
\hline Up & FAM162A & 3 & $2.59 \mathrm{E}-06$ & 1036 & 0.251933 & 0 \\
\hline Up & CDCA7 & 3 & $5.04 \mathrm{E}-04$ & 84502 & 0.25785 & 0 \\
\hline Up & $\mathrm{CNIH} 4$ & 3 & $4.88 \mathrm{E}-04$ & 119748 & 0.191413 & 0 \\
\hline Up & PCOLCE2 & 3 & $9.74 \mathrm{E}-04$ & 174702 & 0.211192 & 0 \\
\hline Up & HLA-H & 2 & 1 & 2 & 1 & 0 \\
\hline Up & APOC1 & 2 & 1.36E-05 & 3656 & 0.190932 & 0 \\
\hline Up & NMB & 2 & 4.87E-04 & 52814 & 0.185926 & 0 \\
\hline Up & PCLAF & 2 & $2.29 \mathrm{E}-05$ & 5430 & 0.254968 & 0 \\
\hline Up & HOXA5 & 2 & 1 & 2 & 1 & 0 \\
\hline Up & IGFBP4 & 2 & 4.87E-04 & 69246 & 0.180357 & 0 \\
\hline Up & EMP3 & 2 & 4.87E-04 & 184158 & 0.15762 & 0 \\
\hline Up & SRPX & 2 & $6.57 \mathrm{E}-06$ & 1448 & 0.233415 & 0 \\
\hline Up & FABP5 & 2 & 4.87E-04 & 80876 & 0.231454 & 0 \\
\hline Up & CD164 & 2 & $2.01 \mathrm{E}-04$ & 38286 & 0.211008 & 0 \\
\hline Up & PDPN & 2 & 1 & 2 & 1 & 0 \\
\hline Up & CMTM3 & 2 & 1 & 2 & 1 & 0 \\
\hline Up & LIPG & 2 & 0 & 0 & 0 & 0 \\
\hline Up & GPNMB & 2 & $1.03 \mathrm{E}-05$ & 1906 & 0.24903 & 0 \\
\hline Up & EVA1A & 2 & $8.20 \mathrm{E}-07$ & 152 & 0.203197 & 0 \\
\hline Up & SLC40A1 & 2 & $8.53 \mathrm{E}-05$ & 16922 & 0.24474 & 0 \\
\hline Up & CLEC2D & 2 & 1 & 2 & 1 & 0 \\
\hline Up & HEBP2 & 2 & 3.60E-07 & 158 & 0.239431 & 0 \\
\hline Up & BRI3 & 1 & 0 & 0 & 1 & 0 \\
\hline Up & TSPAN13 & 1 & 0 & 0 & 0.150889 & 0 \\
\hline Up & TSPAN12 & 1 & 0 & 0 & 0.199621 & 0 \\
\hline Up & COL5A2 & 1 & 0 & 0 & 0.172652 & 0 \\
\hline Up & SAA1 & 1 & 0 & 0 & 1 & 0 \\
\hline Up & $\mathrm{CH} \mid 3 \mathrm{~L} 2$ & 1 & 0 & 0 & 0.227366 & 0 \\
\hline
\end{tabular}


medRxiv preprint doi: https://doi.org/10.1101/2020.12.21.20248616; this version posted December 23, 2020. The copyright holder for this preprint (which was not certified by peer review) is the author/funder, who has granted medRxiv a license to display the preprint in perpetuity. All rights reserved. No reuse allowed without permission.

\begin{tabular}{|c|c|c|c|c|c|c|}
\hline Up & ADM & 1 & 0 & 0 & 1 & 0 \\
\hline Up & OCIAD2 & 1 & 0 & 0 & 0.239417 & 0 \\
\hline Up & GLT8D1 & 1 & 0 & 0 & 1 & 0 \\
\hline Up & METTL7B & 1 & 0 & 0 & 1 & 0 \\
\hline Up & EMILIN2 & 1 & 0 & 0 & 1 & 0 \\
\hline Up & NNMT & 1 & 0 & 0 & 0.196723 & 0 \\
\hline Up & PRPH & 1 & 0 & 0 & 0.175929 & 0 \\
\hline Up & SEC61G & 1 & 0 & 0 & 1 & 0 \\
\hline Up & POSTN & 1 & 0 & 0 & 0.207091 & 0 \\
\hline Up & CA9 & 1 & 0 & 0 & 0.216962 & 0 \\
\hline Up & CD163 & 1 & 0 & 0 & 0.22297 & 0 \\
\hline Up & ZBTB8OS & 1 & 0 & 0 & 1 & 0 \\
\hline Up & RDH10 & 1 & 0 & 0 & 1 & 0 \\
\hline Up & MPLKIP & 1 & 0 & 0 & 1 & 0 \\
\hline Up & GPX8 & 1 & 0 & 0 & 0.203691 & 0 \\
\hline Up & CTHRC1 & 1 & 0 & 0 & 0.209832 & 0 \\
\hline Up & PLIN2 & 1 & 0 & 0 & 1 & 0 \\
\hline Up & TIMP4 & 1 & 0 & 0 & 0.18612 & 0 \\
\hline Up & NOX4 & 1 & 0 & 0 & 0.192526 & 0 \\
\hline Up & HILPDA & 1 & 0 & 0 & 0.154827 & 0 \\
\hline Up & IER3IP1 & 1 & 0 & 0 & 1 & 0 \\
\hline Up & FCGBP & 1 & 0 & 0 & 0.204993 & 0 \\
\hline Down & ARRB1 & 222 & 0.299245 & 11274878 & 0.314014 & $8.30 \mathrm{E}-05$ \\
\hline Down & SNCA & 113 & 0.13603 & 6494358 & 0.291987 & $3.28 \mathrm{E}-04$ \\
\hline Down & ERBB3 & 107 & 0.096221 & 7800068 & 0.2585 & $1.83 \mathrm{E}-04$ \\
\hline Down & PRKCZ & 67 & 0.057416 & 2808426 & 0.267033 & 0.001923 \\
\hline Down & DLG4 & 62 & 0.058577 & 2057566 & 0.259618 & 0.00339 \\
\hline Down & SCN2B & 57 & 0.051144 & 1098524 & 0.243177 & 0.001253 \\
\hline Down & NECAB2 & 57 & 0.050318 & 1946010 & 0.239626 & $6.73 \mathrm{E}-04$ \\
\hline Down & PRKCB & 42 & 0.032753 & 1531646 & 0.247129 & 0 \\
\hline
\end{tabular}


medRxiv preprint doi: https://doi.org/10.1101/2020.12.21.20248616; this version posted December 23, 2020. The copyright holder for this preprint (which was not certified by peer review) is the author/funder, who has granted medRxiv a license to display the preprint in perpetuity. All rights reserved. No reuse allowed without permission.

\begin{tabular}{|c|c|c|c|c|c|c|}
\hline Down & KLC1 & 41 & 0.038803 & 2121170 & 0.246249 & 0.009756 \\
\hline Down & DNM1 & 41 & 0.1099 & 4035506 & 0.296234 & 0.017544 \\
\hline Down & P2RY12 & 41 & 0.033731 & 1037714 & 0.204611 & 0 \\
\hline Down & FGFR3 & 40 & 0.0372 & 830950 & 0.240257 & 0.001422 \\
\hline Down & MAST3 & 39 & 0.032859 & 2033136 & 0.227033 & 0 \\
\hline Down & SH3GL3 & 38 & 0.022541 & 948860 & 0.251184 & 0.019048 \\
\hline Down & STX1A & 37 & 0.02799 & 1253328 & 0.25099 & 0.026891 \\
\hline Down & САМК2B & 37 & 0.04343 & 1610014 & 0.235884 & 0.010084 \\
\hline Down & MAST1 & 37 & 0.031068 & 1364362 & 0.252463 & 0.004505 \\
\hline Down & NEFL & 35 & 0.033757 & 1571428 & 0.254037 & 0.001681 \\
\hline Down & SH3GL2 & 34 & 0.022074 & 789606 & 0.26474 & 0.038306 \\
\hline Down & RALYL & 33 & 0.023538 & 518554 & 0.211694 & 0.006452 \\
\hline Down & LMO3 & 33 & 0.033418 & 1271054 & 0.225708 & 0.005682 \\
\hline Down & PPP2R2C & 33 & 0.028367 & 1595028 & 0.20081 & 0 \\
\hline Down & ARHGEF7 & 32 & 0.036529 & 1794040 & 0.264771 & 0.008065 \\
\hline Down & STXBP1 & 31 & 0.032541 & 1418212 & 0.255374 & 0.021505 \\
\hline Down & SYN1 & 28 & 0.024353 & 916764 & 0.267472 & 0.018462 \\
\hline Down & APLP1 & 28 & 0.024257 & 429482 & 0.249072 & 0 \\
\hline Down & GFOD1 & 28 & 0.019204 & 868372 & 0.190194 & 0 \\
\hline Down & RTP5 & 27 & 0.018739 & 831766 & 0.222103 & 0 \\
\hline Down & PKP4 & 27 & 0.022358 & 994812 & 0.228626 & 0 \\
\hline Down & CDK5R1 & 26 & 0.022769 & 620358 & 0.233494 & 0.006154 \\
\hline Down & PACSIN1 & 26 & 0.016734 & 587328 & 0.243099 & 0.003623 \\
\hline Down & CAMK2A & 26 & 0.027193 & 1188758 & 0.240586 & 0.025362 \\
\hline Down & CBX7 & 25 & 0.028401 & 784128 & 0.22028 & 0 \\
\hline Down & CHGB & 25 & 0.022112 & 586338 & 0.24593 & 0.003333 \\
\hline Down & TF & 23 & 0.022652 & 573562 & 0.2308 & 0 \\
\hline Down & PPP1R16B & 23 & 0.018721 & 325034 & 0.232045 & 0.003953 \\
\hline Down & AMPH & 23 & 0.017902 & 456068 & 0.251627 & 0.033333 \\
\hline Down & SNAP25 & 22 & 0.009756 & 424994 & 0.240156 & 0.052632 \\
\hline
\end{tabular}


medRxiv preprint doi: https://doi.org/10.1101/2020.12.21.20248616; this version posted December 23, 2020. The copyright holder for this preprint (which was not certified by peer review) is the author/funder, who has granted medRxiv a license to display the preprint in perpetuity. All rights reserved. No reuse allowed without permission.

\begin{tabular}{|c|c|c|c|c|c|c|}
\hline Down & RUNDC3A & 22 & 0.01622 & 626548 & 0.189326 & 0 \\
\hline Down & KIAA1107 & 22 & 0.015734 & 565820 & 0.212384 & 0 \\
\hline Down & MAP4 & 21 & 0.01782 & 1145766 & 0.239299 & 0 \\
\hline Down & HSPA2 & 21 & 0.031882 & 1212888 & 0.241734 & 0 \\
\hline Down & EPB41L1 & 21 & 0.020668 & 1515084 & 0.24084 & 0 \\
\hline Down & DNM3 & 20 & 0.012686 & 322436 & 0.246461 & 0.039216 \\
\hline Down & MBP & 20 & 0.017574 & 532696 & 0.255574 & 0.010526 \\
\hline Down & SYT1 & 20 & 0.010022 & 424280 & 0.230032 & 0.052288 \\
\hline Down & GNAO1 & 19 & 0.015028 & 817596 & 0.239853 & 0.005848 \\
\hline Down & ATP2B2 & 19 & 0.025345 & 598378 & 0.220642 & 0 \\
\hline Down & CAMKK2 & 19 & 0.013435 & 526250 & 0.239903 & 0 \\
\hline Down & MPPED1 & 17 & 0.013983 & 485200 & 0.161042 & 0 \\
\hline Down & BRINP1 & 17 & 0.012899 & 403422 & 0.201912 & 0 \\
\hline Down & NEFM & 17 & 0.009499 & 428050 & 0.229385 & 0 \\
\hline Down & RNF130 & 17 & 0.01584 & 332900 & 0.216393 & 0 \\
\hline Down & MAL2 & 17 & 0.012153 & 226228 & 0.219834 & 0 \\
\hline Down & MAPK8IP3 & 17 & 0.00824 & 306938 & 0.226897 & 0.014706 \\
\hline Down & LHX6 & 17 & 0.013213 & 541038 & 0.175354 & 0 \\
\hline Down & GPR37 & 16 & 0.011793 & 322936 & 0.225574 & 0 \\
\hline Down & GABBR1 & 16 & 0.012931 & 617022 & 0.233997 & 0.025 \\
\hline Down & TUBB4A & 16 & 0.015758 & 354884 & 0.22735 & 0 \\
\hline Down & DNAJA4 & 16 & 0.011447 & 270652 & 0.2262 & 0 \\
\hline Down & MAP1A & 16 & 0.01686 & 440326 & 0.241121 & 0 \\
\hline Down & MAP7 & 16 & 0.014152 & 645922 & 0.217279 & 0 \\
\hline Down & CAMK1D & 16 & 0.02205 & 868352 & 0.215433 & 0 \\
\hline Down & DYNC1I1 & 15 & 0.009627 & 329232 & 0.202037 & 0 \\
\hline Down & ADAP1 & 15 & 0.011684 & 258142 & 0.241325 & 0.019048 \\
\hline Down & APOD & 14 & 0.012176 & 277596 & 0.214078 & 0 \\
\hline Down & KLK7 & 14 & 0.009319 & 304520 & 0.177565 & 0 \\
\hline Down & SHANK2 & 14 & 0.007291 & 246346 & 0.247666 & 0.032967 \\
\hline
\end{tabular}


medRxiv preprint doi: https://doi.org/10.1101/2020.12.21.20248616; this version posted December 23, 2020. The copyright holder for this preprint (which was not certified by peer review) is the author/funder, who has granted medRxiv a license to display the preprint in perpetuity. All rights reserved. No reuse allowed without permission.

\begin{tabular}{|c|c|c|c|c|c|c|}
\hline Down & GAD1 & 14 & 0.016156 & 826332 & 0.231668 & 0 \\
\hline Down & $A B C A 2$ & 14 & 0.009983 & 429746 & 0.225395 & 0 \\
\hline Down & NAPB & 14 & 0.006504 & 405924 & 0.2192 & 0 \\
\hline Down & PAK6 & 14 & 0.007389 & 383940 & 0.240232 & 0 \\
\hline Down & KIF5C & 13 & 0.005051 & 235576 & 0.230125 & 0.076923 \\
\hline Down & HTR2A & 13 & 0.010125 & 216080 & 0.211694 & 0 \\
\hline Down & RCAN2 & 13 & 0.006759 & 287940 & 0.180003 & 0 \\
\hline Down & KCNIP3 & 13 & 0.008542 & 310714 & 0.21525 & 0 \\
\hline Down & MAL & 12 & 0.007597 & 366690 & 0.224199 & 0 \\
\hline Down & PTGDS & 12 & 0.00691 & 312530 & 0.236055 & 0 \\
\hline Down & REPS2 & 12 & 0.006915 & 216594 & 0.236079 & 0 \\
\hline Down & STM N2 & 12 & 0.008388 & 294234 & 0.2173 & 0 \\
\hline Down & PLEKHG3 & 11 & 0.004759 & 373098 & 0.200616 & 0 \\
\hline Down & EPHB6 & 11 & 0.007008 & 108334 & 0.182087 & 0 \\
\hline Down & HSPA12A & 11 & 0.005909 & 265148 & 0.187105 & 0 \\
\hline Down & INA & 11 & 0.007688 & 124436 & 0.246968 & 0.018182 \\
\hline Down & STX1B & 11 & 0.00195 & 127750 & 0.228718 & 0.090909 \\
\hline Down & FGF12 & 11 & 0.006679 & 200368 & 0.228993 & 0 \\
\hline Down & GRIN1 & 11 & 0.004477 & 156250 & 0.238001 & 0.055556 \\
\hline Down & DMTN & 11 & 0.006897 & 337378 & 0.240055 & 0 \\
\hline Down & KIF1A & 11 & 0.004578 & 170578 & 0.226335 & 0 \\
\hline Down & SHANK3 & 11 & 0.002049 & 140196 & 0.241657 & 0.036364 \\
\hline Down & DLG2 & 11 & 0.001598 & 166818 & 0.203425 & 0 \\
\hline Down & KLHL3 & 11 & 0.00875 & 328104 & 0.151 & 0 \\
\hline Down & SHTN1 & 10 & 0.004024 & 154544 & 0.187953 & 0 \\
\hline Down & PTPRD & 10 & 0.014121 & 333304 & 0.232068 & 0 \\
\hline Down & NCS1 & 10 & 0.005134 & 376474 & 0.173764 & 0 \\
\hline Down & VSNL1 & 10 & 0.006718 & 467712 & 0.223671 & 0 \\
\hline Down & DLGAP2 & 10 & 0.007987 & 447564 & 0.244375 & 0.022222 \\
\hline Down & RAB40B & 10 & 0.00859 & 189864 & 0.223212 & 0 \\
\hline
\end{tabular}


medRxiv preprint doi: https://doi.org/10.1101/2020.12.21.20248616; this version posted December 23, 2020. The copyright holder for this preprint (which was not certified by peer review) is the author/funder, who has granted medRxiv a license to display the preprint in perpetuity. All rights reserved. No reuse allowed without permission.

\begin{tabular}{|c|c|c|c|c|c|c|}
\hline Down & PPP1R1A & 10 & 0.010051 & 207720 & 0.198296 & 0 \\
\hline Down & CHD5 & 10 & 0.007877 & 211230 & 0.153324 & 0 \\
\hline Down & ATP1B1 & 9 & 0.005207 & 156130 & 0.225842 & 0 \\
\hline Down & CCKBR & 9 & 0.004691 & 247434 & 0.223212 & 0 \\
\hline Down & MATK & 9 & 0.00444 & 189396 & 0.22842 & 0 \\
\hline Down & RAP1GAP & 9 & 0.006173 & 396920 & 0.174428 & 0 \\
\hline Down & sox10 & 9 & 0.003833 & 120652 & 0.189138 & 0 \\
\hline Down & DHRS9 & 9 & 0.006453 & 146034 & 0.184011 & 0 \\
\hline Down & NPTX1 & 9 & 0.00631 & 174320 & 0.156953 & 0 \\
\hline Down & DOCK3 & 9 & 0.00215 & 173482 & 0.229039 & 0 \\
\hline Down & RAB6B & 9 & 0.00642 & 259296 & 0.219919 & 0 \\
\hline Down & ERC2 & 8 & 0.006305 & 164806 & 0.212048 & 0 \\
\hline Down & PLP1 & 8 & 0.004898 & 122954 & 0.214138 & 0 \\
\hline Down & CAPN3 & 8 & 0.003291 & 58262 & 0.213597 & 0.066667 \\
\hline Down & ELAVL3 & 8 & 0.004312 & 96444 & 0.217052 & 0.107143 \\
\hline Down & UGT8 & 8 & 0.004566 & 290206 & 0.181883 & 0 \\
\hline Down & CNDP1 & 8 & 0.006149 & 198894 & 0.155615 & 0 \\
\hline Down & MAP7D2 & 8 & 0.002553 & 91714 & 0.179113 & 0 \\
\hline Down & MUTYH & 8 & 0.004637 & 191474 & 0.175071 & 0 \\
\hline Down & HPCAL4 & 8 & 0.004091 & 266828 & 0.218612 & 0 \\
\hline Down & GLS & 7 & 0.004477 & 93192 & 0.213377 & 0 \\
\hline Down & AK5 & 7 & 0.005255 & 170622 & 0.160025 & 0 \\
\hline Down & RGS4 & 7 & 0.005191 & 234060 & 0.244611 & 0.095238 \\
\hline Down & SYN2 & 7 & 0.00135 & 65348 & 0.226177 & 0.190476 \\
\hline Down & ANKS1B & 7 & 0.003783 & 162066 & 0.230032 & 0 \\
\hline Down & KCNJ4 & 7 & 0.003432 & 77090 & 0.219792 & 0.047619 \\
\hline Down & PIP4K2A & 7 & 0.003342 & 149652 & 0.21442 & 0 \\
\hline Down & LINGO1 & 7 & 0.00616 & 237778 & 0.203661 & 0 \\
\hline Down & KLHL32 & 7 & 0.003315 & 62316 & 0.170721 & 0 \\
\hline Down & OLFM1 & 7 & 0.011361 & 367006 & 0.22453 & 0 \\
\hline
\end{tabular}


medRxiv preprint doi: https://doi.org/10.1101/2020.12.21.20248616; this version posted December 23, 2020. The copyright holder for this preprint (which was not certified by peer review) is the author/funder, who has granted medRxiv a license to display the preprint in perpetuity. All rights reserved. No reuse allowed without permission.

\begin{tabular}{|c|c|c|c|c|c|c|}
\hline Down & GABBR2 & 6 & 0.001296 & 34896 & 0.22122 & 0.2 \\
\hline Down & NPY & 6 & 0.00438 & 146320 & 0.126736 & 0 \\
\hline Down & NDRG2 & 6 & 0.001834 & 42986 & 0.215942 & 0 \\
\hline Down & MOBP & 6 & 0.003715 & 147874 & 0.200193 & 0 \\
\hline Down & KCNAB1 & 6 & 0.002259 & 81552 & 0.23073 & 0 \\
\hline Down & RTN1 & 6 & 0.00438 & 168870 & 0.164741 & 0 \\
\hline Down & ZCCHC12 & 6 & 0.003245 & 66008 & 0.198038 & 0 \\
\hline Down & RIMS2 & 6 & 0.001835 & 68800 & 0.232921 & 0 \\
\hline Down & SEMA4D & 6 & 0.002629 & 178470 & 0.181941 & 0 \\
\hline Down & ANO3 & 6 & 0.003928 & 96916 & 0.203515 & 0 \\
\hline Down & TMOD2 & 6 & 0.003658 & 86702 & 0.19566 & 0 \\
\hline Down & CNTNAP2 & 6 & 0.003238 & 108012 & 0.206912 & 0 \\
\hline Down & PTPRT & 5 & $5.40 \mathrm{E}-04$ & 34072 & 0.228534 & 0 \\
\hline Down & PPFIA2 & 5 & 0.004404 & 125812 & 0.192587 & 0 \\
\hline Down & SNAP91 & 5 & $9.61 \mathrm{E}-05$ & 16034 & 0.216784 & 0 \\
\hline Down & CELF2 & 5 & 0.002943 & 75450 & 0.22573 & 0 \\
\hline Down & SYP & 5 & $7.49 \mathrm{E}-04$ & 40020 & 0.22011 & 0 \\
\hline Down & CNP & 5 & 0.001952 & 57266 & 0.17762 & 0 \\
\hline Down & GABRB3 & 5 & 0.006223 & 206948 & 0.169465 & 0 \\
\hline Down & RGS7 & 5 & 0.00219 & 120382 & 0.195676 & 0 \\
\hline Down & GNG3 & 5 & 0.003404 & 83208 & 0.194078 & 0 \\
\hline Down & HPCA & 5 & $9.13 \mathrm{E}-04$ & 53922 & 0.216784 & 0 \\
\hline Down & GAD2 & 5 & 0.001197 & 40718 & 0.184816 & 0 \\
\hline Down & KCNMA1 & 5 & 0.001753 & 94402 & 0.162488 & 0 \\
\hline Down & CELF3 & 5 & 0.003505 & 221348 & 0.156351 & 0 \\
\hline Down & ABLIM2 & 5 & 0.002006 & 42058 & 0.195492 & 0 \\
\hline Down & RAB11FIP4 & 5 & 0.006662 & 188546 & 0.200123 & 0 \\
\hline Down & CAMKV & 5 & $2.12 \mathrm{E}-04$ & 11296 & 0.217673 & 0 \\
\hline Down & MYRIP & 5 & 0.001795 & 67700 & 0.214501 & 0 \\
\hline Down & NRGN & 5 & 0.003457 & 128186 & 0.200228 & 0 \\
\hline
\end{tabular}


medRxiv preprint doi: https://doi.org/10.1101/2020.12.21.20248616; this version posted December 23, 2020. The copyright holder for this preprint (which was not certified by peer review) is the author/funder, who has granted medRxiv a license to display the preprint in perpetuity. All rights reserved. No reuse allowed without permission.

\begin{tabular}{|c|c|c|c|c|c|c|}
\hline Down & MAGEE1 & 5 & 0.001012 & 52882 & 0.177662 & 0 \\
\hline Down & PPP1R1B & 5 & 0.004492 & 131660 & 0.178636 & 0 \\
\hline Down & RAPGEF5 & 5 & 1 & 20 & 1 & 0 \\
\hline Down & PEG3 & 5 & 0.002465 & 144102 & 0.199301 & 0 \\
\hline Down & PLEKHB1 & 5 & 0.001921 & 78134 & 0.186083 & 0 \\
\hline Down & AGAP1 & 5 & 0.002654 & 97416 & 0.152239 & 0 \\
\hline Down & PDE2A & 4 & $8.77 \mathrm{E}-04$ & 14580 & 0.210134 & 0 \\
\hline Down & CPLX1 & 4 & $2.65 \mathrm{E}-04$ & 6254 & 0.212542 & 0.5 \\
\hline Down & KCNQ3 & 4 & 0.001216 & 46298 & 0.199895 & 0 \\
\hline Down & BCAS1 & 4 & 8.77E-04 & 32938 & 0.194161 & 0 \\
\hline Down & RGS11 & 4 & 0.001112 & 48222 & 0.195458 & 0 \\
\hline Down & ATP6V1G2 & 4 & 0.002629 & 63414 & 0.194625 & 0 \\
\hline Down & PDYN & 4 & 1 & 12 & 1 & 0 \\
\hline Down & VIP & 4 & 0.004065 & 97706 & 0.191697 & 0 \\
\hline Down & GABRG2 & 4 & 8.77E-04 & 38662 & 0.156975 & 0 \\
\hline Down & CALB2 & 4 & $3.64 \mathrm{E}-05$ & 4472 & 0.216743 & 0 \\
\hline Down & ASPA & 4 & $8.77 \mathrm{E}-04$ & 32820 & 0.116022 & 0 \\
\hline Down & GRM3 & 4 & 0.002601 & 85176 & 0.200228 & 0 \\
\hline Down & DOK6 & 4 & 0.00444 & 154144 & 0.234623 & 0 \\
\hline Down & PPP1R3F & 4 & 0.002151 & 58042 & 0.201966 & 0 \\
\hline Down & KLK6 & 4 & 0.002629 & 71898 & 0.143063 & 0 \\
\hline Down & SLC30A3 & 4 & 0.9 & 18 & 0.833333 & 0 \\
\hline Down & SULT4A1 & 4 & 0.001105 & 58032 & 0.214259 & 0 \\
\hline Down & SLC6A15 & 4 & 0.001559 & 69598 & 0.182451 & 0 \\
\hline Down & KCNK4 & 4 & 1 & 2 & 1 & 0 \\
\hline Down & MTMR7 & 4 & $8.77 \mathrm{E}-04$ & 19672 & 0.117948 & 0 \\
\hline Down & SFRP1 & 4 & 0.002955 & 102192 & 0.167585 & 0 \\
\hline Down & CDKL2 & 4 & 0.002629 & 67740 & 0.20665 & 0 \\
\hline Down & NELL1 & 4 & 0.003577 & 123452 & 0.226649 & 0 \\
\hline Down & AGAP3 & 4 & 0.004407 & 160394 & 0.214864 & 0 \\
\hline
\end{tabular}


medRxiv preprint doi: https://doi.org/10.1101/2020.12.21.20248616; this version posted December 23, 2020. The copyright holder for this preprint (which was not certified by peer review) is the author/funder, who has granted medRxiv a license to display the preprint in perpetuity. All rights reserved. No reuse allowed without permission.

\begin{tabular}{|c|c|c|c|c|c|c|}
\hline Down & EFHD1 & 4 & 1 & 12 & 1 & 0 \\
\hline Down & CNTNAP4 & 4 & 0.002629 & 80814 & 0.174242 & 0 \\
\hline Down & CACNA1I & 4 & 0.001946 & 97888 & 0.177055 & 0 \\
\hline Down & PNMA3 & 4 & 0.001774 & 70226 & 0.201164 & 0 \\
\hline Down & PLLP & 4 & 0.002005 & 45752 & 0.197797 & 0 \\
\hline Down & ZNF536 & 3 & 1 & 6 & 1 & 0 \\
\hline Down & $\mathrm{FA} 2 \mathrm{H}$ & 3 & 0.001102 & 20302 & 0.186128 & 0 \\
\hline Down & KCNQ2 & 3 & $9.05 \mathrm{E}-04$ & 34786 & 0.172073 & 0 \\
\hline Down & CDS1 & 3 & 0.001753 & 33622 & 0.195693 & 0 \\
\hline Down & LY6H & 3 & 0.001753 & 31514 & 0.162905 & 0 \\
\hline Down & DDN & 3 & $9.26 \mathrm{E}-04$ & 44630 & 0.183522 & 0 \\
\hline Down & TSPOAP1 & 3 & 1 & 6 & 1 & 0 \\
\hline Down & CCK & 3 & 0.001753 & 83294 & 0.182553 & 0 \\
\hline Down & CHRM3 & 3 & 0.002014 & 54652 & 0.199023 & 0 \\
\hline Down & ITPKA & 3 & $3.93 \mathrm{E}-04$ & 15862 & 0.194708 & 0 \\
\hline Down & SLC1A2 & 3 & $2.45 \mathrm{E}-04$ & 11574 & 0.220472 & 0 \\
\hline Down & GRIN2C & 3 & $8.98 \mathrm{E}-04$ & 14756 & 0.217424 & 0.333333 \\
\hline Down & OPCML & 3 & $6.20 \mathrm{E}-04$ & 25720 & 0.194758 & 0 \\
\hline Down & ARHGAP44 & 3 & 0.001753 & 37378 & 0.153211 & 0 \\
\hline Down & TTLL7 & 3 & 0.001616 & 47796 & 0.223408 & 0 \\
\hline Down & PDZD4 & 3 & 0.002481 & 79798 & 0.224574 & 0 \\
\hline Down & SCN2A & 3 & 0.001753 & 30734 & 0.177967 & 0 \\
\hline Down & ANKRD24 & 3 & 0.001174 & 34950 & 0.197404 & 0 \\
\hline Down & RAPGEF4 & 3 & 0.001083 & 18302 & 0.205348 & 0 \\
\hline Down & FOSB & 3 & $9.50 \mathrm{E}-04$ & 28400 & 0.200828 & 0 \\
\hline Down & SGSM1 & 3 & 1 & 6 & 1 & 0 \\
\hline Down & FBXL16 & 3 & 0.001753 & 87138 & 0.149633 & 0 \\
\hline Down & DOCK9 & 3 & $9.27 \mathrm{E}-04$ & 40200 & 0.185507 & 0 \\
\hline Down & STMN4 & 3 & 0.001753 & 45262 & 0.158634 & 0 \\
\hline Down & ADARB2 & 3 & 0.001753 & 116842 & 0.163337 & 0 \\
\hline
\end{tabular}


medRxiv preprint doi: https://doi.org/10.1101/2020.12.21.20248616; this version posted December 23, 2020. The copyright holder for this preprint (which was not certified by peer review) is the author/funder, who has granted medRxiv a license to display the preprint in perpetuity. All rights reserved. No reuse allowed without permission.

\begin{tabular}{|c|c|c|c|c|c|c|}
\hline Down & OGDHL & 3 & $1.00 \mathrm{E}-03$ & 28438 & 0.19993 & 0 \\
\hline Down & NSG2 & 3 & 1 & 6 & 1 & 0 \\
\hline Down & ST8SIA3 & 2 & 0 & 0 & 0 & 0 \\
\hline Down & SYT7 & 2 & $2.11 \mathrm{E}-04$ & 6072 & 0.1921 & 0 \\
\hline Down & SYNGR1 & 2 & 0.003503 & 93152 & 0.158008 & 0 \\
\hline Down & KIAA0513 & 2 & 1 & 2 & 1 & 0 \\
\hline Down & CACNG3 & 2 & $1.51 \mathrm{E}-04$ & 6176 & 0.130589 & 0 \\
\hline Down & CA11 & 2 & $2.40 \mathrm{E}-05$ & 1120 & 0.179961 & 0 \\
\hline Down & CPNE6 & 2 & 0.006122 & 199010 & 0.190305 & 0 \\
\hline Down & FAM153A & 2 & 1 & 2 & 1 & 0 \\
\hline Down & GABRB1 & 2 & 1 & 2 & 1 & 0 \\
\hline Down & MAG & 2 & $8.77 \mathrm{E}-04$ & 21140 & 0.194558 & 0 \\
\hline Down & KCNJ6 & 2 & 1 & 2 & 1 & 0 \\
\hline Down & IL1RAPL1 & 2 & 0.001379 & 13992 & 0.179875 & 0 \\
\hline Down & RELN & 2 & 8.77E-04 & 26248 & 0.17099 & 0 \\
\hline Down & CNTN2 & 2 & $8.35 \mathrm{E}-04$ & 32912 & 0.227827 & 0 \\
\hline Down & CRYM & 2 & $3.27 \mathrm{E}-04$ & 10428 & 0.195559 & 0 \\
\hline Down & ASIC2 & 2 & $8.77 \mathrm{E}-04$ & 28542 & 0.156179 & 0 \\
\hline Down & KIAA0319 & 2 & 8.77E-04 & 19690 & 0.162372 & 0 \\
\hline Down & UNC5A & 2 & 8.77E-04 & 28648 & 0.151551 & 0 \\
\hline Down & DAAM2 & 2 & $1.77 \mathrm{E}-04$ & 9332 & 0.185251 & 0 \\
\hline Down & CPEB3 & 2 & 1 & 2 & 1 & 0 \\
\hline Down & SYNPR & 2 & $8.77 \mathrm{E}-04$ & 23294 & 0.120129 & 0 \\
\hline Down & KIFC2 & 2 & 1 & 2 & 1 & 0 \\
\hline Down & VSTM2L & 2 & 8.77E-04 & 26602 & 0.184368 & 0 \\
\hline Down & S1PR5 & 2 & $8.77 \mathrm{E}-04$ & 23402 & 0.162766 & 0 \\
\hline Down & SLC12A5 & 2 & $7.62 \mathrm{E}-06$ & 968 & 0.211028 & 0 \\
\hline Down & MYRF & 2 & 0 & 0 & 0 & 0 \\
\hline Down & CLDN10 & 2 & $8.77 \mathrm{E}-04$ & 19690 & 0.162372 & 0 \\
\hline Down & CDH18 & 2 & 1 & 2 & 1 & 0 \\
\hline
\end{tabular}


medRxiv preprint doi: https://doi.org/10.1101/2020.12.21.20248616; this version posted December 23, 2020. The copyright holder for this preprint (which was not certified by peer review) is the author/funder, who has granted medRxiv a license to display the preprint in perpetuity. All rights reserved. No reuse allowed without permission.

\begin{tabular}{|c|c|c|c|c|c|c|}
\hline Down & RAP1GAP2 & 2 & 4.04E-06 & 302 & 0.202647 & 0 \\
\hline Down & RGS7BP & 2 & $8.77 \mathrm{E}-04$ & 33498 & 0.153159 & 0 \\
\hline Down & PPP4R4 & 2 & 8.77E-04 & 21902 & 0.201876 & 0 \\
\hline Down & SH2D5 & 2 & $9.38 \mathrm{E}-05$ & 5996 & 0.216393 & 0 \\
\hline Down & BRSK1 & 2 & $8.77 \mathrm{E}-04$ & 32710 & 0.17321 & 0 \\
\hline Down & PHACTR3 & 2 & $8.77 \mathrm{E}-04$ & 28146 & 0.135653 & 0 \\
\hline Down & FAIM2 & 2 & $8.77 \mathrm{E}-04$ & 17602 & 0.158227 & 0 \\
\hline Down & SYT4 & 2 & 8.77E-04 & 21916 & 0.138637 & 0 \\
\hline Down & NRIP3 & 2 & 1 & 2 & 1 & 0 \\
\hline Down & CALY & 2 & $8.77 \mathrm{E}-04$ & 19142 & 0.193011 & 0 \\
\hline Down & ARPP21 & 2 & $8.77 \mathrm{E}-04$ & 48378 & 0.194924 & 0 \\
\hline Down & BSN & 2 & $3.02 \mathrm{E}-05$ & 356 & 0.197609 & 0 \\
\hline Down & CARNS1 & 1 & 0 & 0 & 1 & 0 \\
\hline Down & RIMBP2 & 1 & 0 & 0 & 1 & 0 \\
\hline Down & NTSR2 & 1 & 0 & 0 & 1 & 0 \\
\hline Down & FAM153B & 1 & 0 & 0 & 0.666667 & 0 \\
\hline Down & SLC6A1 & 1 & 0 & 0 & 0.200651 & 0 \\
\hline Down & VIPR1 & 1 & 0 & 0 & 0.170836 & 0 \\
\hline Down & ADCY1 & 1 & 0 & 0 & 1 & 0 \\
\hline Down & $\mathrm{ABCC} 8$ & 1 & 0 & 0 & 1 & 0 \\
\hline Down & TEF & 1 & 0 & 0 & 0.148764 & 0 \\
\hline Down & NNAT & 1 & 0 & 0 & 1 & 0 \\
\hline Down & TMEM155 & 1 & 0 & 0 & 0.145231 & 0 \\
\hline Down & SV2B & 1 & 0 & 0 & 1 & 0 \\
\hline Down & NRSN1 & 1 & 0 & 0 & 0.120116 & 0 \\
\hline Down & FSTL5 & 1 & 0 & 0 & 1 & 0 \\
\hline Down & $\mathrm{JPH} 3$ & 1 & 0 & 0 & 0.149938 & 0 \\
\hline Down & PDE1A & 1 & 0 & 0 & 1 & 0 \\
\hline Down & $A D C Y 2$ & 1 & 0 & 0 & 0.192945 & 0 \\
\hline Down & ENPP2 & 1 & 0 & 0 & 0.195861 & 0 \\
\hline
\end{tabular}


medRxiv preprint doi: https://doi.org/10.1101/2020.12.21.20248616; this version posted December 23, 2020. The copyright holder for this preprint (which was not certified by peer review) is the author/funder, who has granted medRxiv a license to display the preprint in perpetuity. All rights reserved. No reuse allowed without permission.

\begin{tabular}{|c|c|c|c|c|c|c|}
\hline Down & TMEM132D & 1 & 0 & 0 & 0.135637 & 0 \\
\hline Down & MOG & 1 & 0 & 0 & 1 & 0 \\
\hline Down & SOWAHA & 1 & 0 & 0 & 0.152954 & 0 \\
\hline Down & GJC2 & 1 & 0 & 0 & 0.162441 & 0 \\
\hline Down & PAQR6 & 1 & 0 & 0 & 0.171788 & 0 \\
\hline Down & KIAA1549L & 1 & 0 & 0 & 0.2128 & 0 \\
\hline Down & KNDC1 & 1 & 0 & 0 & 0.177676 & 0 \\
\hline Down & NAV3 & 1 & 0 & 0 & 1 & 0 \\
\hline Down & CYP4X1 & 1 & 0 & 0 & 0.164551 & 0 \\
\hline Down & SVOP & 1 & 0 & 0 & 0.171788 & 0 \\
\hline Down & ТТС9В & 1 & 0 & 0 & 0.18584 & 0 \\
\hline Down & ERMN & 1 & 0 & 0 & 0.222298 & 0 \\
\hline Down & SERPINI1 & 1 & 0 & 0 & 1 & 0 \\
\hline Down & STOX2 & 1 & 0 & 0 & 1 & 0 \\
\hline Down & TAGLN3 & 1 & 0 & 0 & 1 & 0 \\
\hline Down & RIMS3 & 1 & 0 & 0 & 1 & 0 \\
\hline Down & PLEKHH1 & 1 & 0 & 0 & 1 & 0 \\
\hline Down & PPM1H & 1 & 0 & 0 & 0.202019 & 0 \\
\hline Down & SLC8A2 & 1 & 0 & 0 & 1 & 0 \\
\hline Down & SCN8A & 1 & 0 & 0 & 1 & 0 \\
\hline Down & CYP46A1 & 1 & 0 & 0 & 1 & 0 \\
\hline Down & PCLO & 1 & 0 & 0 & 0.175246 & 0 \\
\hline Down & CAMTA1 & 1 & 0 & 0 & 0.162441 & 0 \\
\hline
\end{tabular}

Table 7 miRNA - target gene interaction table

\begin{tabular}{cccccccc} 
Regulation & Target Genes & Degree & MicroRNA & Regulation & Target Genes & Degree & MicroRNA \\
\hline Up & SOD2 & 257 & hsa-mir-6077 & Down & SVOP & 107 & hsa-mir-3972 \\
Up & WEE1 & 167 & hsa-mir-4457 & Down & KCNJ6 & 90 & hsa-mir-4287 \\
Up & G3BP1 & 158 & hsa-mir-4437 & Down & SYT7 & 75 & hsa-mir-4441
\end{tabular}


medRxiv preprint doi: https://doi.org/10.1101/2020.12.21.20248616; this version posted December 23, 2020. The copyright holder for this preprint (which was not certified by peer review) is the author/funder, who has granted medRxiv a license to display the preprint in perpetuity. All rights reserved. No reuse allowed without permission.

\begin{tabular}{|c|c|c|c|c|c|c|c|}
\hline Up & CNBP & 153 & hsa-mir-4260 & Down & RAB11FIP4 & 73 & hsa-mir-3176 \\
\hline Up & HMGB 1 & 143 & hsa-mir-5193 & Down & NPTX1 & 69 & hsa-mir-3119 \\
\hline Up & HNRNPA1 & 139 & hsa-mir-8073 & Down & SYNGR1 & 64 & hsa-mir-4314 \\
\hline Up & MTHFD2 & 122 & hsa-mir-8071 & Down & KIAA0513 & 63 & hsa-mir- 1253 \\
\hline Up & MPLKIP & 121 & hsa-mir-1827 & Down & GABRB1 & 61 & hsa-mir-3646 \\
\hline Up & TMEM167A & 112 & hsa-mir-5681a & Down & $\begin{array}{l}\text { PALM2- } \\
\text { AKAP2 }\end{array}$ & 59 & hsa-mir-4741 \\
\hline Up & VEGFA & 108 & hsa-mir-4483 & Down & TMEM151B & 59 & hsa-mir-572 \\
\hline Up & MYC & 103 & hsa-mir-4289 & Down & PPP1R16B & 54 & hsa-mir-3670 \\
\hline Up & MCTS1 & 101 & hsa-mir-3199 & Down & CHRM3 & 54 & hsa-mir-4522 \\
\hline Up & PTMA & 100 & hsa-mir-4801 & Down & CPEB3 & 53 & hsa-mir-4328 \\
\hline Up & YY1 & 99 & hsa-mir-4522 & Down & PACSIN1 & 53 & hsa-mir-4459 \\
\hline Up & CLIC4 & 97 & hsa-mir-5695 & Down & MAST3 & 50 & hsa-mir-4506 \\
\hline Up & PPIC & 92 & hsa-mir-6131 & Down & MAP7 & 48 & hsa-mir-4295 \\
\hline Up & ID4 & 91 & hsa-mir-3161 & Down & TMOD2 & 48 & hsa-mir-1825 \\
\hline Up & HMGB2 & 89 & hsa-mir-3924 & Down & PRKCB & 47 & hsa-mir-6074 \\
\hline Up & KPNA2 & 83 & hsa-mir-4302 & Down & NCS1 & 47 & hsa-mir-4679 \\
\hline Up & TXNL1 & 80 & hsa-mir-3659 & Down & ACBD7 & 46 & hsa-mir-3123 \\
\hline Up & $\mathrm{E} 2 \mathrm{~F} 2$ & 78 & hsa-mir-8063 & Down & SCN2B & 46 & hsa-mir-6088 \\
\hline Up & RAN & 78 & hsa-mir-4491 & Down & TMEM151A & 46 & hsa-mir-5193 \\
\hline Up & ZFP36L1 & 77 & hsa-mir-1321 & Down & SLC1A2 & 45 & hsa-mir-4284 \\
\hline Up & $\mathrm{ADM}$ & 77 & hsa-mir-3188 & Down & PEX5L & 45 & hsa-mir-548v \\
\hline Up & SERPINH1 & 76 & hsa-mir-4492 & Down & TEF & 44 & hsa-mir-4262 \\
\hline Up & BIRC5 & 75 & hsa-mir-3652 & Down & SV2B & 44 & hsa-mir-4518 \\
\hline Up & LAMC1 & 74 & hsa-mir-3973 & Down & STOX2 & 42 & hsa-mir-3611 \\
\hline Up & RPS15A & 73 & hsa-mir-3135b & Down & SRRM4 & 42 & hsa-mir-4448 \\
\hline Up & HIF1A & 72 & hsa-mir-4499 & Down & PLLP & 41 & hsa-mir-3975 \\
\hline Up & CCNB1 & 71 & hsa-mir-6089 & Down & PIP4K2A & 40 & hsa-mir-4683 \\
\hline Up & LIPG & 68 & hsa-mir-4435 & Down & SEMA4D & 40 & hsa-mir-3973 \\
\hline Up & MELK & 67 & hsa-mir-891b & Down & ELAVL3 & 40 & hsa-mir-6071 \\
\hline Up & BUB1 & 67 & hsa-mir-644a & Down & EPB41L1 & 40 & hsa-mir-6133 \\
\hline
\end{tabular}


medRxiv preprint doi: https://doi.org/10.1101/2020.12.21.20248616; this version posted December 23, 2020. The copyright holder for this preprint (which was not certified by peer review) is the author/funder, who has granted medRxiv a license to display the preprint in perpetuity. All rights reserved. No reuse allowed without permission.

\begin{tabular}{|c|c|c|c|c|c|c|c|}
\hline Up & HNRNPC & 66 & hsa-mir-5090 & Down & SLC12A5 & 38 & hsa-mir-4479 \\
\hline Up & RAB13 & 65 & hsa-mir-7977 & Down & ARRB1 & 38 & hsa-mir-1470 \\
\hline Up & RAP1B & 64 & hsa-mir-4496 & Down & CNDP1 & 38 & hsa-mir-891b \\
\hline Up & OCIAD2 & 63 & hsa-mir-4294 & Down & UGT8 & 37 & hsa-mir-4497 \\
\hline Up & EEF1A1 & 62 & hsa-mir-4784 & Down & STX1B & 36 & hsa-mir-6072 \\
\hline Up & STC2 & 62 & hsa-mir-8060 & Down & RIMS3 & 34 & hsa-mir-4286 \\
\hline Up & CD164 & 60 & hsa-mir-5688 & Down & PLEKHH1 & 34 & hsa-mir-7977 \\
\hline Up & SLC16A10 & 60 & hsa-mir-4430 & Down & SLITRK4 & 34 & hsa-mir-4643 \\
\hline Up & EIF4E & 59 & hsa-mir-4422 & Down & FBXL16 & 34 & hsa-mir-8057 \\
\hline Up & TFPI & 59 & hsa-mir-3649 & Down & DDN & 33 & hsa-mir-663b \\
\hline Up & CDK2 & 59 & hsa-mir-4270 & Down & SEC14L5 & 33 & hsa-mir-6131 \\
\hline Up & RPL14 & 58 & hsa-mir-548an & Down & PPM1H & 32 & hsa-mir-4308 \\
\hline Up & CAV1 & 58 & hsa-mir-4277 & Down & PTPRT & 32 & hsa-mir-5096 \\
\hline Up & CEP55 & 57 & hsa-mir-3658 & Down & NMNAT2 & 31 & hsa-mir-1275 \\
\hline Up & TAGLN2 & 55 & hsa-mir-4773 & Down & GABRB3 & 31 & hsa-mir-548ac \\
\hline Up & IGFBP4 & 55 & hsa-mir-9500 & Down & RCAN2 & 31 & hsa-mir-548aw \\
\hline Up & AURKA & 53 & hsa-mir-4476 & Down & PTPRD & 30 & hsa-mir-4791 \\
\hline Up & VCAM1 & 53 & hsa-mir-5694 & Down & NRIP3 & 30 & hsa-mir-548ah-5p \\
\hline $\mathrm{Up}$ & ETS1 & 53 & hsa-mir-507 & Down & PAK6 & 30 & hsa-mir-3686 \\
\hline Up & CDK1 & 52 & hsa-mir-599 & Down & ADARB2 & 30 & hsa-mir-4420 \\
\hline Up & BTG3 & 50 & hsa-mir-5087 & Down & MAPK8IP3 & 30 & hsa-mir-3196 \\
\hline Up & CD44 & 49 & hsa-mir-5696 & Down & SOWAHA & 30 & hsa-mir-9500 \\
\hline Up & IGF2BP3 & 49 & hsa-mir-892b & Down & KLHL3 & 29 & hsa-mir-603 \\
\hline Up & TFAP2A & 49 & hsa-mir-761 & Down & CAMKV & 29 & hsa-mir-4714-5p \\
\hline Up & HMOX1 & 47 & hsa-mir-4443 & Down & CAMK2A & 29 & hsa-mir- 8082 \\
\hline Up & PPIA & 47 & hsa-mir-610 & Down & ERBB3 & 28 & hsa-mir-4698 \\
\hline Up & SUMO2 & 47 & hsa-mir-4263 & Down & RAP1GAP2 & 28 & hsa-mir-4525 \\
\hline Up & APH1A & 47 & hsa-mir-4450 & Down & AGAP1 & 28 & hsa-mir-6077 \\
\hline Up & RDH10 & 46 & hsa-mir-5787 & Down & CELF2 & 27 & hsa-mir-548aa \\
\hline Up & RPL18A & 46 & hsa-mir-421 & Down & CREG2 & 27 & hsa-mir-3919 \\
\hline Up & TGIF1 & 46 & hsa-mir-2117 & Down & CAMKK2 & 25 & hsa-mir-4330 \\
\hline
\end{tabular}


medRxiv preprint doi: https://doi.org/10.1101/2020.12.21.20248616; this version posted December 23, 2020. The copyright holder for this preprint (which was not certified by peer review) is the author/funder, who has granted medRxiv a license to display the preprint in perpetuity. All rights reserved. No reuse allowed without permission.

\begin{tabular}{|c|c|c|c|c|c|c|c|}
\hline Up & AK4 & 45 & hsa-mir-4484 & Down & VSNL1 & 25 & hsa-mir-599 \\
\hline Up & IER3IP1 & 45 & hsa-mir-577 & Down & CDK5R1 & 25 & hsa-mir-548ac \\
\hline Up & HSPA4 & 44 & hsa-mir-4282 & Down & IL1RAPL1 & 25 & hsa-mir-663b \\
\hline Up & SMS & 44 & hsa-mir-4257 & Down & RAB40B & 24 & hsa-mir-1304-3p \\
\hline Up & OIP5 & 42 & hsa-mir-4480 & Down & FBXO41 & 24 & hsa-mir-7855-5p \\
\hline Up & RPS27A & 42 & hsa-mir-643 & Down & SNAP25 & 24 & hsa-mir-4480 \\
\hline Up & COL4A1 & 41 & hsa-mir-7-2-3p & Down & SFRP1 & 24 & hsa-mir-8089 \\
\hline Up & EMP1 & 41 & hsa-mir-4481 & Down & KCNK12 & 24 & hsa-mir-3650 \\
\hline Up & HAT1 & 40 & hsa-let-7f-1-3p & Down & NRGN & 24 & hsa-mir-665 \\
\hline Up & SRPX2 & 40 & hsa-mir-2052 & Down & DOK6 & 23 & hsa-mir-3941 \\
\hline Up & COL8A1 & 39 & hsa-mir-3939 & Down & CDKL2 & 23 & hsa-mir-4635 \\
\hline Up & CKS2 & 39 & hsa-mir-5697 & Down & GABRG2 & 23 & hsa-mir-4667-3p \\
\hline Up & HLA-A & 39 & hsa-mir-6133 & Down & MOG & 23 & hsa-mir-4428 \\
\hline Up & HEBP2 & 39 & hsa-mir-5693 & Down & MAG & 23 & hsa-mir-6129 \\
\hline Up & CLEC2D & 38 & hsa-mir-429 & Down & ADCY2 & 22 & hsa-mir-4430 \\
\hline Up & CKAP2L & 38 & hsa-mir-4689 & Down & ST8SIA3 & 21 & hsa-mir-4469 \\
\hline Up & F2R & 37 & hsa-mir-4706 & Down & OTUD7A & 21 & hsa-mir- 8080 \\
\hline Up & CYP51A1 & 37 & hsa-mir-302f & Down & RAB6B & 21 & hsa-mir-6130 \\
\hline Up & CCNA2 & 36 & hsa-mir-548aw & Down & LHPP & 21 & hsa-mir-5093 \\
\hline Up & RPS4X & 34 & hsa-mir-4526 & Down & NECAB1 & 21 & hsa-mir-4282 \\
\hline Up & HOXA5 & 34 & hsa-mir-4295 & Down & KIF5C & 20 & hsa-mir-603 \\
\hline Up & GNA13 & 33 & hsa-mir-3924 & Down & TTLL7 & 20 & hsa-mir-4455 \\
\hline Up & STEAP3 & 33 & hsa-mir-760 & Down & C1orf115 & 20 & hsa-mir-4520-3p \\
\hline Up & CD99 & 32 & hsa-mir-3199 & Down & SGSM1 & 20 & hsa-mir-548s \\
\hline Up & HOXC6 & 32 & hsa-mir-4419a & Down & BSN & 20 & hsa-mir-3155a \\
\hline Up & EMILIN2 & 31 & hsa-mir-4458 & Down & SLC30A3 & 20 & hsa-mir-4270 \\
\hline $\mathrm{Up}$ & GINS2 & 31 & hsa-mir-4478 & Down & DAAM2 & 19 & hsa-mir-4439 \\
\hline Up & ID2 & 31 & hsa-mir-298 & Down & PDZD4 & 19 & hsa-mir-6777-5p \\
\hline Up & ZBTB8OS & 29 & hsa-mir-4744 & Down & PPP1R1A & 19 & hsa-mir-612 \\
\hline Up & TPT1 & 29 & hsa-mir-3142 & Down & HSPA2 & 18 & hsa-mir- 8088 \\
\hline Up & TPI1 & 29 & hsa-mir-3202 & Down & TMEFF2 & 18 & hsa-mir-7641 \\
\hline
\end{tabular}


medRxiv preprint doi: https://doi.org/10.1101/2020.12.21.20248616; this version posted December 23, 2020. The copyright holder for this preprint (which was not certified by peer review) is the author/funder, who has granted medRxiv a license to display the preprint in perpetuity. All rights reserved. No reuse allowed without permission.

\begin{tabular}{|c|c|c|c|c|c|c|c|}
\hline Up & F3 & 29 & hsa-mir-4422 & Down & $\mathrm{CBX} 7$ & 17 & hsa-mir-548t-3p \\
\hline Up & COL5A1 & 29 & hsa-mir-4434 & Down & SLC6A17 & 17 & hsa-mir-1273d \\
\hline Up & PDPN & 29 & hsa-mir-940 & Down & PLP1 & 17 & hsa-mir-4666b \\
\hline Up & HSPD1 & 28 & hsa-mir-3118 & Down & TUBB4A & 17 & hsa-mir-3974 \\
\hline Up & HMGN1 & 28 & hsa-mir-548ax & Down & LY6H & 17 & hsa-mir-3188 \\
\hline Up & SEC24D & 28 & hsa-mir-640 & Down & PLEKHG3 & 17 & hsa-mir-4668-3p \\
\hline Up & GAPDH & 27 & hsa-mir-644a & Down & ARHGEF7 & 16 & hsa-mir-3919 \\
\hline Up & PCNA & 27 & hsa-mir-548u & Down & SH2D5 & 16 & hsa-mir-7977 \\
\hline Up & RPS3 & 27 & hsa-mir-6844 & Down & ABCA2 & 15 & hsa-mir-320e \\
\hline Up & VIM & 27 & hsa-mir-6124 & Down & KCNIP3 & 15 & hsa-mir-557 \\
\hline Up & COL1A2 & 27 & hsa-mir-4424 & Down & SYT4 & 15 & hsa-mir-4719 \\
\hline Up & ITGA5 & 26 & hsa-mir-1912 & Down & MPPED1 & 15 & hsa-mir-378g \\
\hline Up & NEDD9 & 26 & hsa-mir-520h & Down & KCNQ3 & 15 & hsa-mir-4685-3p \\
\hline Up & WTAP & 26 & hsa-mir-1299 & Down & CPLX1 & 15 & hsa-mir-3960 \\
\hline Up & RPL9 & 25 & hsa-mir-548n & Down & CAMTA1 & 14 & hsa-mir-129-5p \\
\hline Up & FSTL1 & 25 & hsa-mir-206 & Down & BRSK1 & 14 & hsa-mir-6825-5p \\
\hline Up & RPS2 & 25 & hsa-mir-3943 & Down & CARNS1 & 14 & hsa-mir-147a \\
\hline Up & RHOQ & 25 & hsa-mir- 8485 & Down & CNTNAP2 & 14 & hsa-mir-548u \\
\hline Up & CSRP2 & 25 & hsa-mir-4451 & Down & SYP & 14 & hsa-mir-5692c \\
\hline Up & XPR1 & 25 & hsa-mir-5189-3p & Down & FAIM2 & 14 & hsa-mir-4288 \\
\hline Up & GPX8 & 25 & hsa-mir-5700 & Down & MAP4 & 13 & hsa-mir-3655 \\
\hline Up & CA12 & 24 & hsa-mir-5011-5p & Down & DOCK9 & 13 & hsa-mir-4477a \\
\hline Up & RPS27 & 24 & hsa-mir-6088 & Down & SCN8A & 13 & hsa-mir-329-3p \\
\hline Up & RPLP1 & 24 & hsa-mir-4436b-5p & Down & GPIHBP1 & 13 & hsa-mir-8071 \\
\hline Up & ADAMTS9 & 24 & hsa-mir-7-1-3p & Down & GNG3 & 13 & hsa-mir-765 \\
\hline Up & HJURP & 24 & hsa-mir-298 & Down & NKX6-2 & 13 & hsa-mir-6883-5p \\
\hline Up & SAA1 & 24 & hsa-mir-8085 & Down & ENPP2 & 12 & hsa-mir-107 \\
\hline Up & SLC39A14 & 23 & hsa-mir-137 & Down & ANKRD24 & 12 & hsa-mir-5191 \\
\hline Up & FTH1 & 23 & hsa-mir-548m & Down & CACNA1E & 12 & hsa-mir-892b \\
\hline Up & CENPA & 23 & hsa-mir-1276 & Down & PNMA3 & 12 & hsa-mir-6875-3p \\
\hline $\mathrm{Up}$ & ID3 & 23 & hsa-mir-5002-5p & Down & JAKMIP3 & 12 & hsa-mir-4421 \\
\hline
\end{tabular}


medRxiv preprint doi: https://doi.org/10.1101/2020.12.21.20248616; this version posted December 23, 2020. The copyright holder for this preprint (which was not certified by peer review) is the author/funder, who has granted medRxiv a license to display the preprint in perpetuity. All rights reserved. No reuse allowed without permission.

\begin{tabular}{|c|c|c|c|c|c|c|c|}
\hline Up & AKAP12 & 22 & hsa-mir-592 & Down & $\mathrm{SCN} 3 \mathrm{~B}$ & 12 & hsa-mir-4537 \\
\hline Up & CDCA7 & 22 & hsa-mir-550a-5p & Down & VIPR1 & 12 & hsa-mir-4507 \\
\hline Up & KIFC1 & 22 & hsa-mir-532-3p & Down & RAPGEF4 & 11 & hsa-mir-20b-5p \\
\hline $\mathrm{Up}$ & TGFBI & 22 & hsa-mir- 6070 & Down & REPS2 & 11 & hsa-mir-3656 \\
\hline Up & HNRNPK & 22 & hsa-mir-5571-5p & Down & LHX6 & 11 & hsa-mir-1273f \\
\hline Up & RPS7 & 22 & hsa-mir-5003-3p & Down & PARM1 & 11 & hsa-mir-6835-3p \\
\hline Up & SERPINA3 & 22 & hsa-mir-7111-3p & Down & PDE1A & 11 & hsa-mir-519e-5p \\
\hline $\mathrm{Up}$ & NXT2 & 21 & hsa-mir-5002-5p & Down & KIFC2 & 11 & hsa-mir-626 \\
\hline Up & RPN2 & 21 & hsa-mir-219b-3p & Down & KLHL32 & 11 & hsa-mir-3200-5p \\
\hline $\mathrm{Up}$ & RPS26 & 21 & hsa-mir-596 & Down & JPH3 & 11 & hsa-mir-455-3p \\
\hline Up & LYZ & 21 & hsa-mir-7703 & Down & KIAA0319 & 11 & hsa-mir-6756-3p \\
\hline Up & RPL15 & 21 & hsa-mir-589-3p & Down & GABRA5 & 11 & hsa-mir-4503 \\
\hline Up & DLGAP5 & 21 & hsa-mir-520c-3p & Down & MOBP & 10 & hsa-mir-3941 \\
\hline Up & S100A11 & 20 & hsa-mir-3609 & Down & ASPA & 10 & hsa-mir-203a-3p \\
\hline Up & NCAPG & 20 & hsa-mir-575 & Down & DNAJA4 & 10 & hsa-mir-6083 \\
\hline Up & SLBP & 19 & hsa-mir-8064 & Down & TMEM130 & 10 & hsa-mir-3177-5p \\
\hline Up & RPL7 & 19 & hsa-mir-205-3p & Down & ADAP1 & 10 & hsa-mir-6500-3p \\
\hline Up & MAD2L1 & 18 & hsa-mir-3977 & Down & SHANK2 & 10 & hsa-mir-4469 \\
\hline $\mathrm{Up}$ & CDKN3 & 18 & hsa-mir-6890-3p & Down & LINGO2 & 10 & hsa-mir-660-3p \\
\hline $\mathrm{Up}$ & CENPK & 18 & hsa-mir-3680-3p & Down & MAL2 & 10 & hsa-mir-6739-3p \\
\hline Up & PRSS23 & 18 & hsa-mir-466 & Down & PAQR8 & 10 & hsa-mir-507 \\
\hline Up & HILPDA & 18 & hsa-mir-5683 & Down & SLC6A1 & 10 & hsa-mir-6833-3p \\
\hline Up & CD68 & 18 & hsa-mir-4667-3p & Down & PPP1R3F & 10 & hsa-mir-548t-5p \\
\hline $\mathrm{Up}$ & CKAP2 & 17 & hsa-mir-6755-5p & Down & KIF1A & 9 & hsa-mir-652-3p \\
\hline Up & USP8 & 17 & hsa-mir-8066 & Down & GFOD1 & 9 & hsa-mir-100-5p \\
\hline Up & TOP2A & 17 & hsa-mir-708-5p & Down & FGFR3 & 9 & hsa-mir-4764-5p \\
\hline Up & RPS28 & 17 & hsa-mir-4504 & Down & PCLO & 9 & hsa-mir-7-1-3p \\
\hline $\mathrm{Up}$ & PRC1 & 17 & hsa-mir-2277-3p & Down & KCTD16 & 9 & hsa-mir-377-3p \\
\hline $\mathrm{Up}$ & STC1 & 17 & hsa-mir-6881-3p & Down & RIMBP2 & 9 & hsa-mir-371a-5p \\
\hline Up & UQCRH & 16 & hsa-mir-608 & Down & BCAS1 & 9 & hsa-mir-933 \\
\hline $\mathrm{Up}$ & KIF11 & 15 & hsa-mir-6507-5p & Down & OPCML & 9 & hsa-mir-3149 \\
\hline
\end{tabular}


medRxiv preprint doi: https://doi.org/10.1101/2020.12.21.20248616; this version posted December 23, 2020. The copyright holder for this preprint (which was not certified by peer review) is the author/funder, who has granted medRxiv a license to display the preprint in perpetuity. All rights reserved. No reuse allowed without permission.

\begin{tabular}{|c|c|c|c|c|c|c|c|}
\hline Up & NAMPT & 15 & hsa-mir-4422 & Down & CES4A & 9 & hsa-mir-4673 \\
\hline Up & TSPAN12 & 15 & hsa-mir-6718-5p & Down & GABBR1 & 8 & hsa-mir-20a-5p \\
\hline Up & TSC22D1 & 15 & hsa-mir-6856-5p & Down & KRT222 & 8 & hsa-mir-5007-3p \\
\hline Up & LOX & 15 & hsa-mir-6888-3p & Down & BHLHE22 & 8 & hsa-mir-6749-3p \\
\hline Up & FAM162A & 15 & hsa-mir-3160-3p & Down & DLGAP2 & 8 & hsa-mir-544a \\
\hline Up & PRRX1 & 15 & hsa-mir-378j & Down & KCNAB1 & 8 & hsa-mir-3606-3p \\
\hline Up & UHRF1 & 14 & hsa-mir-221-3p & Down & KCNJ4 & 8 & hsa-mir-510-3p \\
\hline Up & VKORC1 & 13 & hsa-mir-326 & Down & RGS11 & 8 & hsa-mir-7114-3p \\
\hline Up & RPL7A & 13 & hsa-mir-1303 & Down & CAMK1D & 7 & hsa-mir-6719-3p \\
\hline Up & ECT2 & 13 & hsa-mir-3591-5p & Down & CLDN10 & 7 & hsa-mir-6810-3p \\
\hline Up & PRCP & 13 & hsa-mir-6895-3p & Down & RIMS2 & 6 & hsa-mir-3065-3p \\
\hline Up & C21 orf62 & 13 & hsa-mir-4685-3p & Down & SYNPR & 6 & hsa-mir-877-3p \\
\hline Up & РPP2CB & 13 & hsa-mir-4753-3p & Down & $\mathrm{CNP}$ & 6 & hsa-mir-345-5p \\
\hline Up & LAMA4 & 13 & hsa-mir-103b & Down & MYT1L & 6 & hsa-mir-4503 \\
\hline Up & VOPP1 & 13 & hsa-mir-1273f & Down & GABRA2 & 6 & hsa-mir-545-5p \\
\hline Up & HSPA1A & 12 & hsa-mir-223-5p & Down & CKMT1A & 6 & hsa-mir-924 \\
\hline Up & BNIP3L & 12 & hsa-mir-7109-3p & Down & GABBR2 & 6 & hsa-mir-450b-5p \\
\hline Up & KDELR2 & 12 & hsa-mir-600 & Down & HTR2A & 6 & hsa-mir-4329 \\
\hline Up & COL1A1 & 12 & hsa-mir-4800-5p & Down & CBLN2 & 6 & hsa-mir-7109-3p \\
\hline Up & RPL23 & 12 & hsa-mir-3200-3p & Down & PLEKHB1 & 5 & hsa-mir-7-5p \\
\hline Up & RPL23A & 12 & hsa-mir-892c-5p & Down & INA & 5 & hsa-mir-376a-3p \\
\hline Up & CENPF & 11 & hsa-mir-1226-3p & Down & ZFR2 & 5 & hsa-mir-6886-5p \\
\hline Up & GPX7 & 11 & hsa-mir-5187-5p & Down & DLG4 & 5 & hsa-mir-4668-5p \\
\hline Up & RPS4Y 1 & 11 & hsa-mir-3617-5p & Down & RGS4 & 5 & hsa-mir-6867-5p \\
\hline Up & TSPAN13 & 11 & hsa-mir-944 & Down & SYT13 & 5 & hsa-mir-4775 \\
\hline Up & PITX1 & 11 & hsa-mir-6749-3p & Down & STMN4 & 5 & hsa-mir-4279 \\
\hline Up & NNMT & 11 & hsa-mir-6500-3p & Down & MTMR7 & 5 & hsa-mir-103a-2-5p \\
\hline Up & IL1RAP & 11 & hsa-mir-548c-3p & Down & PDYN & 5 & hsa-mir-548av-3p \\
\hline Up & C15orf48 & 11 & hsa-mir-7978 & Down & MUTYH & 5 & hsa-mir-4320 \\
\hline Up & IGFBP3 & 11 & hsa-mir-1909-5p & Down & LGI3 & 5 & hsa-mir-4496 \\
\hline Up & AURKB & 10 & hsa-mir-148a-3p & Down & GPR83 & 4 & hsa-mir-181a-5p \\
\hline
\end{tabular}


medRxiv preprint doi: https://doi.org/10.1101/2020.12.21.20248616; this version posted December 23, 2020. The copyright holder for this preprint (which was not certified by peer review) is the author/funder, who has granted medRxiv a license to display the preprint in perpetuity. All rights reserved. No reuse allowed without permission.

\begin{tabular}{|c|c|c|c|c|c|c|c|}
\hline Up & RPS10 & 10 & hsa-mir-3176 & Down & ADCY 1 & 4 & hsa-mir-331-3p \\
\hline Up & LPL & 10 & hsa-mir-544a & Down & FGF12 & 4 & hsa-mir-590-5p \\
\hline Up & EIF3M & 10 & hsa-mir-3662 & Down & STXBP1 & 4 & hsa-mir-378a-3p \\
\hline Up & PTGES3 & 10 & hsa-mir-4709-5p & Down & ANO3 & 4 & hsa-mir-18a-3p \\
\hline Up & RPS8 & 10 & hsa-mir-100-5p & Down & KIAA1107 & 4 & hsa-mir-7-2-3p \\
\hline Up & SAP30 & 10 & hsa-mir-5096 & Down & GPR37 & 4 & hsa-mir-215-5p \\
\hline Up & $\mathrm{CNIH} 4$ & 9 & hsa-mir-301b-3p & Down & DOCK3 & 4 & hsa-mir-486-5p \\
\hline Up & RPS3A & 9 & hsa-mir-342-3p & Down & SYT1 & 4 & hsa-mir-8064 \\
\hline Up & TUBA1C & 9 & hsa-mir-296-3p & Down & CUX2 & 4 & hsa-mir-4455 \\
\hline Up & MMP9 & 9 & hsa-mir-338-3p & Down & KNDC1 & 4 & hsa-mir-550b-2-5p \\
\hline Up & TNFRSF12A & 9 & hsa-mir-6780b-3p & Down & LINGO1 & 4 & hsa-mir-6077 \\
\hline Up & UBE2C & 9 & hsa-mir-671-5p & Down & NETO1 & 4 & hsa-mir-5692a \\
\hline Up & CD93 & 9 & hsa-mir-499b-3p & Down & ERMN & 3 & hsa-mir-10a-5p \\
\hline Up & MS4A4A & 9 & hsa-mir-4700-3p & Down & SLC6A15 & 3 & hsa-mir-23a-3p \\
\hline Up & COL3A1 & 8 & hsa-mir-455-3p & Down & KCNQ2 & 3 & hsa-mir-148b-3p \\
\hline Up & WLS & 8 & hsa-mir-6793-3p & Down & ST18 & 3 & hsa-mir-451b \\
\hline Up & RPS12 & 8 & hsa-mir-18a-3p & Down & STX1A & 3 & hsa-mir-34a-5p \\
\hline Up & EEF1B2 & 8 & hsa-mir-548ag & Down & PKP4 & 3 & hsa-mir-218-5p \\
\hline Up & PLOD2 & 8 & hsa-mir-136-5p & Down & CCKBR & 3 & hsa-mir-152-3p \\
\hline Up & KDM3A & 8 & hsa-mir-30d-5p & Down & ZNF365 & 3 & hsa-mir-223-3p \\
\hline Up & ZFAND6 & 8 & hsa-mir-580-5p & Down & RELN & 3 & hsa-mir-138-5p \\
\hline Up & RPL29 & 8 & hsa-mir-652-3p & Down & CDS1 & 3 & hsa-mir-324-5p \\
\hline Up & RPS11 & 8 & hsa-mir-5691 & Down & STXBP6 & 3 & hsa-mir-4539 \\
\hline Up & ANXA2 & 7 & hsa-mir-133a-3p & Down & NEFM & 2 & hsa-let-7a-5p \\
\hline Up & CTHRC1 & 7 & hsa-mir-630 & Down & DNM3 & 2 & hsa-let-7b-5p \\
\hline Up & PTTG1 & 7 & hsa-mir-655-3p & Down & ADAD2 & 2 & hsa-mir-16-5p \\
\hline Up & RPL31 & 7 & hsa-let-7d-5p & Down & NAPB & 2 & hsa-mir-19b-3p \\
\hline Up & PLAU & 7 & hsa-mir-19a-3p & Down & $\mathrm{TF}$ & 2 & hsa-mir-19a-3p \\
\hline Up & COL5A2 & 7 & hsa-mir-29c-3p & Down & SLITRK1 & 2 & hsa-mir-24-1-5p \\
\hline Up & CLIC1 & 7 & hsa-mir-25-3p & Down & ARHGAP44 & 2 & hsa-mir-30c-5p \\
\hline Up & TTK & 7 & hsa-mir-376a-5p & Down & HSPA12A & 2 & hsa-mir-339-5p \\
\hline
\end{tabular}


medRxiv preprint doi: https://doi.org/10.1101/2020.12.21.20248616; this version posted December 23, 2020. The copyright holder for this preprint (which was not certified by peer review) is the author/funder, who has granted medRxiv a license to display the preprint in perpetuity. All rights reserved. No reuse allowed without permission.

\begin{tabular}{|c|c|c|c|c|c|c|c|}
\hline Up & $\mathrm{C} 1 \mathrm{~S}$ & 7 & hsa-mir-4701-5p & Down & PAQR6 & 2 & hsa-mir-26b-5p \\
\hline Up & ESM1 & 7 & hsa-mir-1260a & Down & PLCH2 & 2 & hsa-mir-331-3p \\
\hline Up & CAST & 6 & hsa-mir-150-5p & Down & SH3GL2 & 2 & hsa-mir-330-3p \\
\hline Up & LARP4 & 6 & hsa-mir-148b-3p & Down & SNAP91 & 2 & hsa-mir-26b-5p \\
\hline Up & ASPM & 6 & hsa-mir-218-5p & Down & СКМТ1В & 2 & hsa-mir-92a-3p \\
\hline Up & CCNB2 & 6 & hsa-let-7c-5p & Down & ATP1B1 & 2 & hsa-mir-192-5p \\
\hline Up & LAMB1 & 6 & hsa-mir-500a-3p & Down & MAP6D1 & 2 & hsa-mir-215-5p \\
\hline Up & GBP2 & 6 & hsa-mir-6806-5p & Down & PDE2A & 2 & hsa-mir-192-5p \\
\hline Up & SP3 & 6 & hsa-mir-27a-3p & Down & RAP1GAP & 2 & hsa-mir-215-5p \\
\hline Up & PRDX4 & 6 & hsa-mir-3619-5p & Down & CPNE6 & 2 & hsa-mir-197-3p \\
\hline Up & P4HA1 & 6 & hsa-mir-877-3p & Down & KLC1 & 2 & hsa-mir-34a-5p \\
\hline Up & TMSB10 & 5 & hsa-mir-27b-3p & Down & NNAT & 2 & hsa-mir-708-5p \\
\hline Up & KIF20A & 5 & hsa-mir-23a-3p & Down & GLS & 2 & hsa-mir-7-5p \\
\hline Up & ANXA1 & 5 & hsa-mir-30a-5p & Down & SNCA & 2 & hsa-mir-153-3p \\
\hline Up & FBXO5 & 5 & hsa-mir-151a-3p & Down & KCNMA1 & 2 & hsa-mir-211-5p \\
\hline Up & GMNN & 5 & hsa-mir-449a & Down & FOSB & 2 & hsa-mir-224-5p \\
\hline Up & RPS18 & 5 & hsa-mir-193b-3p & Down & CLCA4 & 2 & hsa-mir-128-3p \\
\hline Up & SNX7 & 5 & hsa-mir-9-5p & Down & NDRG2 & 2 & hsa-mir-650 \\
\hline $\mathrm{Up}$ & HMMR & 5 & hsa-mir-215-5p & Down & ABCC8 & 2 & hsa-mir-136-5p \\
\hline Up & СCT8 & 4 & hsa-mir-301a-3p & Down & AGAP3 & 2 & hsa-mir-149-5p \\
\hline Up & CHPT1 & 4 & hsa-mir-16-5p & Down & ZDHHC11 & 2 & hsa-mir-375 \\
\hline Up & MDK & 4 & hsa-mir-17-5p & Down & DLG2 & 2 & hsa-mir-148b-3p \\
\hline Up & HOXB2 & 4 & hsa-mir-605-5p & Down & MAST1 & 2 & hsa-mir-335-5p \\
\hline Up & CDCA5 & 4 & hsa-mir-18a-5p & Down & MAP1A & 2 & hsa-mir-338-3p \\
\hline Up & TMEM45A & 4 & hsa-mir-19b-3p & Down & APLP1 & 2 & hsa-mir-484 \\
\hline Up & СМТМ3 & 4 & hsa-mir-24-3p & Down & OGDHL & 2 & hsa-mir-130b-5p \\
\hline Up & NSMAF & 4 & hsa-mir-92a-3p & Down & PEG3 & 2 & hsa-mir-412-3p \\
\hline Up & PCOLCE2 & 4 & hsa-mir-182-5p & Down & CHD5 & 2 & hsa-mir-744-5p \\
\hline Up & PDLIM1 & 4 & hsa-mir-504-5p & Down & OPALIN & 2 & hsa-mir-186-3p \\
\hline Up & TIMP1 & 4 & hsa-mir-320a & Down & JPH4 & 1 & hsa-let-7a-5p \\
\hline Up & PYGL & 4 & hsa-mir-10a-5p & Down & AMPH & 1 & hsa-let-7b-5p \\
\hline
\end{tabular}


medRxiv preprint doi: https://doi.org/10.1101/2020.12.21.20248616; this version posted December 23, 2020. The copyright holder for this preprint (which was not certified by peer review) is the author/funder, who has granted medRxiv a license to display the preprint in perpetuity. All rights reserved. No reuse allowed without permission.

\begin{tabular}{|c|c|c|c|c|c|c|c|}
\hline Up & RPS29 & 4 & hsa-mir-484 & Down & CDH18 & 1 & hsa-let-7e-5p \\
\hline Up & $\mathrm{ABCC} 3$ & 4 & hsa-mir-197-3p & Down & KLK6 & 1 & hsa-let-7f-5p \\
\hline Up & MMP7 & 4 & hsa-mir-3939 & Down & SERPINI1 & 1 & hsa-mir-21-5p \\
\hline Up & SPOCD1 & 4 & hsa-mir-4290 & Down & CA11 & 1 & hsa-mir-26b-5p \\
\hline Up & PBK & 4 & hsa-mir-216b-3p & Down & CACNG3 & 1 & hsa-mir-26b-5p \\
\hline Up & FPR3 & 4 & hsa-mir-718 & Down & $\mathrm{CH} 25 \mathrm{H}$ & 1 & hsa-mir-26b-5p \\
\hline Up & TNFAIP6 & 4 & hsa-mir-2052 & Down & SCN2A & 1 & hsa-mir-26b-5p \\
\hline Up & EMP3 & 3 & hsa-mir-1-1 & Down & TAGLN3 & 1 & hsa-mir-26b-5p \\
\hline Up & LGALS1 & 3 & hsa-mir-22-3p & Down & NAV3 & 1 & hsa-mir-29a-3p \\
\hline Up & RPS13 & 3 & hsa-let-7a-5p & Down & NEFL & 1 & hsa-mir-32-5p \\
\hline Up & IFI30 & 3 & hsa-let-7c-3p & Down & GARNL3 & 1 & hsa-mir-92a-3p \\
\hline Up & RPS25 & 3 & hsa-mir-92a-3p & Down & SH3GL3 & 1 & hsa-mir-196a-5p \\
\hline Up & SOCS2 & 3 & hsa-mir-7-5p & Down & PRDM8 & 1 & hsa-mir-7-5p \\
\hline Up & CFI & 3 & hsa-mir-181a-5p & Down & ATP6V1G2 & 1 & hsa-mir-181a-5p \\
\hline Up & $\mathrm{A} 2 \mathrm{M}$ & 3 & hsa-mir-122-5p & Down & XIST & 1 & hsa-mir-210-3p \\
\hline Up & CLK1 & 3 & hsa-mir-186-5p & Down & ANKS1B & 1 & hsa-mir-124-3p \\
\hline Up & HLA-G & 3 & hsa-mir-148a-3p & Down & ARPP21 & 1 & hsa-mir-124-3p \\
\hline Up & NES & 3 & hsa-mir-432-5p & Down & S1PR5 & 1 & hsa-mir-124-3p \\
\hline Up & PAPSS1 & 2 & hsa-mir-100-5p & Down & UNC13C & 1 & hsa-mir-128-3p \\
\hline Up & SBDS & 2 & hsa-mir-590-3p & Down & PPP2R2C & 1 & hsa-mir-142-3p \\
\hline Up & BRI3 & 2 & hsa-let-7e-5p & Down & CAMK2B & 1 & hsa-mir-186-5p \\
\hline Up & DCAF13 & 2 & hsa-mir-149-5p & Down & RNF130 & 1 & hsa-mir-320a \\
\hline Up & RPL6 & 2 & hsa-mir-16-5p & Down & UNC5A & 1 & hsa-mir-320a \\
\hline Up & SLC16A3 & 2 & hsa-mir-98-5p & Down & CHGB & 1 & hsa-mir-375 \\
\hline Up & CCL20 & 2 & hsa-mir-21-5p & Down & SLC32A1 & 1 & hsa-mir-148b-3p \\
\hline Up & BCHE & 2 & hsa-mir-26b-5p & Down & CACNA1I & 1 & hsa-mir-324-3p \\
\hline Up & C1QTNF1 & 2 & hsa-mir-335-5p & Down & CALB2 & 1 & hsa-mir-335-5p \\
\hline Up & CA9 & 2 & hsa-mir-98-5p & Down & DYNC1I1 & 1 & hsa-mir-335-5p \\
\hline Up & IL13RA2 & 2 & hsa-mir-148b-3p & Down & FAM189A1 & 1 & hsa-mir-335-5p \\
\hline Up & MGP & 2 & hsa-mir-26b-5p & Down & GJB6 & 1 & hsa-mir-335-5p \\
\hline Up & PPP1R14B & 2 & hsa-mir-615-3p & Down & GRM3 & 1 & hsa-mir-335-5p \\
\hline
\end{tabular}


medRxiv preprint doi: https://doi.org/10.1101/2020.12.21.20248616; this version posted December 23, 2020. The copyright holder for this preprint (which was not certified by peer review) is the author/funder, who has granted medRxiv a license to display the preprint in perpetuity. All rights reserved. No reuse allowed without permission.

\begin{tabular}{|c|c|c|c|c|c|c|c|}
\hline $\mathrm{Up}$ & SEC61G & 2 & hsa-mir-1260b & Down & HHATL & 1 & hsa-mir-335-5p \\
\hline $\mathrm{Up}$ & UAP1 & 2 & hsa-mir-30a-5p & Down & KCNH3 & 1 & hsa-mir-335-5p \\
\hline $\mathrm{Up}$ & NEK2 & 2 & hsa-mir-128-3p & Down & KCNK4 & 1 & hsa-mir-335-5p \\
\hline $\mathrm{Up}$ & CCZ1 & 2 & hsa-mir-215-5p & Down & KLK7 & 1 & hsa-mir-335-5p \\
\hline $\mathrm{Up}$ & PHLDA1 & 2 & hsa-mir-375 & Down & LMO3 & 1 & hsa-mir-335-5p \\
\hline $\mathrm{Up}$ & GNG10 & 2 & hsa-mir-1301-3p & Down & MATK & 1 & hsa-mir-335-5p \\
\hline $\mathrm{Up}$ & PLEKHA4 & 2 & hsa-mir-124-3p & Down & NKAIN2 & 1 & hsa-mir-335-5p \\
\hline $\mathrm{Up}$ & TUBB6 & 2 & hsa-mir-92b-3p & Down & NPY & 1 & hsa-mir-335-5p \\
\hline $\mathrm{Up}$ & ANGPT2 & 2 & hsa-mir-542-3p & Down & PPFIA2 & 1 & hsa-mir-335-5p \\
\hline $\mathrm{Up}$ & IGFBP2 & 2 & hsa-mir-126-3p & Down & PTGDS & 1 & hsa-mir-335-5p \\
\hline $\mathrm{Up}$ & OSTC & 2 & hsa-mir-377-3p & Down & RGS7BP & 1 & hsa-mir-335-5p \\
\hline $\mathrm{Up}$ & $\mathrm{CDC} 45$ & 2 & hsa-mir-455-3p & Down & SLC17A7 & 1 & hsa-mir-335-5p \\
\hline $\mathrm{Up}$ & LGALS3 & 2 & hsa-mir-744-5p & Down & SLC39A12 & 1 & hsa-mir-335-5p \\
\hline $\mathrm{Up}$ & NUSAP1 & 1 & hsa-let-7b-5p & Down & SYN1 & 1 & hsa-mir-335-5p \\
\hline $\mathrm{Up}$ & GLT8D1 & 1 & hsa-mir-16-5p & Down & SYN2 & 1 & hsa-mir-335-5p \\
\hline Up & LSM5 & 1 & hsa-mir-16-5p & Down & TMEM155 & 1 & hsa-mir-335-5p \\
\hline Up & CASP4 & 1 & hsa-mir-26b-5p & Down & VIP & 1 & hsa-mir-335-5p \\
\hline $\mathrm{Up}$ & EIF1AY & 1 & hsa-mir-26b-5p & Down & VSTM2L & 1 & hsa-mir-335-5p \\
\hline $\mathrm{Up}$ & GGH & 1 & hsa-mir-26b-5p & Down & EFHD1 & 1 & hsa-mir-193b-3p \\
\hline $\mathrm{Up}$ & GPNMB & 1 & hsa-mir-26b-5p & Down & ITPKA & 1 & hsa-mir-193b-3p \\
\hline $\mathrm{Up}$ & SRPX & 1 & hsa-mir-26b-5p & Down & CAPN3 & 1 & hsa-mir-544a \\
\hline $\mathrm{Up}$ & TMSB15A & 1 & hsa-mir-26b-5p & Down & DNM1 & 1 & hsa-mir-615-3p \\
\hline $\mathrm{Up}$ & S100A10 & 1 & hsa-mir-100-5p & Down & $\mathrm{ZCCHC} 12$ & 1 & hsa-mir-615-3p \\
\hline $\mathrm{Up}$ & ALPK2 & 1 & hsa-mir-214-3p & Down & RASAL1 & 1 & hsa-mir-421 \\
\hline $\mathrm{Up}$ & ACTR3 & 1 & hsa-mir-124-3p & Down & PRODH & 1 & hsa-mir-23b-5p \\
\hline $\mathrm{Up}$ & GBP1 & 1 & hsa-mir-124-3p & Down & MBP & 1 & hsa-mir-127-5p \\
\hline $\mathrm{Up}$ & PRPH & 1 & hsa-mir-124-3p & Down & MAP7D2 & 1 & hsa-mir-505-5p \\
\hline $\mathrm{Up}$ & SNRPG & 1 & hsa-mir-320a & Down & HPCAL4 & 1 & hsa-mir-744-5p \\
\hline $\mathrm{Up}$ & H19 & 1 & hsa-mir-375 & Down & ANO4 & 1 & hsa-mir-378c \\
\hline Up & NOX4 & 1 & hsa-mir-148b-3p & & & & \\
\hline $\mathrm{Up}$ & AMY1A & 1 & hsa-mir-335-5p & & & & \\
\hline
\end{tabular}


medRxiv preprint doi: https://doi.org/10.1101/2020.12.21.20248616; this version posted December 23, 2020. The copyright holder for this preprint (which was not certified by peer review) is the author/funder, who has granted medRxiv a license to display the preprint in perpetuity.

All rights reserved. No reuse allowed without permission.

$\begin{array}{cccc}\text { Up } & \text { APOC1 } & 1 & \text { hsa-mir-335-5p } \\ \text { Up } & \text { CHI3L2 } & 1 & \text { hsa-mir-335-5p } \\ \text { Up } & \text { GDF15 } & 1 & \text { hsa-mir-335-5p } \\ \text { Up } & \text { RNASE2 } & 1 & \text { hsa-mir-335-5p } \\ \text { Up } & \text { TNC } & 1 & \text { hsa-mir-335-5p } \\ \text { Up } & \text { LYPD1 } & 1 & \text { hsa-mir-423-3p } \\ \text { Up } & \text { SLC40A1 } & 1 & \text { hsa-mir-485-3p } \\ \text { Up } & \text { TPX2 } & 1 & \text { hsa-mir-193b-3p }\end{array}$

Degree - No of miRNA interact with target gene. We taken any one miRNA in table.

Table $8 \mathrm{TF}$ - target gene interaction table

\begin{tabular}{|c|c|c|c|c|c|c|c|}
\hline Regulation & TF & Degree & Target Gene & Regulation & TF & Degree & Target Gene \\
\hline Up & SOX2 & 225 & ABCC3 & Down & SUZ12 & 234 & ABCA2 \\
\hline Up & NANOG & 180 & VKORC1 & Down & REST & 201 & МOBP \\
\hline Up & SPI1 & 171 & MCTS1 & Down & EGR1 & 198 & PLEKHG3 \\
\hline Up & E2F1 & 167 & TNFRSF12A & Down & SOX2 & 188 & TTLL7 \\
\hline Up & POU5F1 & 155 & C15orf 48 & Down & $\mathrm{AR}$ & 178 & CAPN3 \\
\hline Up & RUNX1 & 148 & CDCA5 & Down & MTF2 & 161 & DYNC1I1 \\
\hline Up & KLF4 & 139 & AURKB & Down & STAT3 & 153 & LY6H \\
\hline Up & HNF4A & 135 & UAP1 & Down & MYC & 150 & PTPRD \\
\hline Up & TP63 & 132 & ITGA5 & Down & TP53 & 142 & UNC13C \\
\hline Up & $\mathrm{AR}$ & 130 & ID4 & Down & POU5F1 & 141 & HPCA \\
\hline Up & FLI1 & 127 & $\mathrm{~F} 2 \mathrm{R}$ & Down & TCF4 & 139 & KLK7 \\
\hline Up & EGR1 & 126 & NAMPT & Down & TP63 & 134 & CKMT1A \\
\hline Up & STAT3 & 120 & LAMA4 & Down & NANOG & 134 & RGS4 \\
\hline Up & PPARG & 119 & MYC & Down & MITF & 132 & CHGB \\
\hline Up & CREM & 119 & PTGES3 & Down & SMAD4 & 129 & NNAT \\
\hline Up & TP53 & 113 & COL8A1 & Down & RUNX1 & 125 & GLS \\
\hline Up & CREB1 & 106 & MMP9 & Down & HNF4A & 125 & TAGLN3 \\
\hline Up & TRIM28 & 105 & VOPP1 & Down & SIN3B & 124 & SST \\
\hline Up & MITF & 102 & CA12 & Down & RCOR3 & 116 & SYT1 \\
\hline Up & E2F4 & 100 & ANXA2 & Down & FLI1 & 105 & $\mathrm{CCK}$ \\
\hline
\end{tabular}


medRxiv preprint doi: https://doi.org/10.1101/2020.12.21.20248616; this version posted December 23, 2020. The copyright holder for this preprint (which was not certified by peer review) is the author/funder, who has granted medRxiv a license to display the preprint in perpetuity. All rights reserved. No reuse allowed without permission.

\begin{tabular}{|c|c|c|c|c|c|c|c|}
\hline Up & SMARCA4 & 97 & COL4A1 & Down & SPI1 & 101 & HPCAL4 \\
\hline Up & KDM5B & 94 & CDK1 & Down & EZH2 & 100 & SPOCK1 \\
\hline Up & GATA2 & 94 & FPR3 & Down & TET1 & 99 & ARHGAP44 \\
\hline Up & SMAD4 & 93 & CEP55 & Down & GATA1 & 99 & JAKMIP3 \\
\hline Up & PPARD & 93 & RPL29 & Down & JARID2 & 93 & LHX6 \\
\hline Up & TCF3 & 89 & HOXB2 & Down & RNF2 & 90 & KCNMA1 \\
\hline Up & FOXP1 & 88 & S100A3 & Down & RUNX2 & 88 & MOG \\
\hline Up & TAL1 & 88 & YY1 & Down & GATA2 & 87 & APOD \\
\hline Up & ZFX & 87 & OSTC & Down & SETDB1 & 82 & PDYN \\
\hline Up & ASH2L & 86 & BTG3 & Down & SMARCA4 & 82 & ST18 \\
\hline Up & TFAP2C & 86 & PDPN & Down & SRY & 81 & NDRG2 \\
\hline Up & CUX1 & 85 & CENPF & Down & TCF3 & 80 & GFOD1 \\
\hline Up & SETDB1 & 83 & H19 & Down & PPARD & 80 & NEFM \\
\hline Up & SALL4 & 81 & EMP3 & Down & CREM & 79 & DNAJA4 \\
\hline Up & KLF1 & 75 & $\mathrm{E} 2 \mathrm{~F} 2$ & Down & SOX9 & 79 & NELL1 \\
\hline Up & SMAD3 & 75 & ETS1 & Down & TRIM28 & 78 & LMO3 \\
\hline Up & SIN3B & 75 & TUBA1C & Down & KLF4 & 77 & HNF4A \\
\hline Up & EOMES & 74 & AKAP12 & Down & CREB1 & 77 & MPPED1 \\
\hline Up & SUZ12 & 74 & COL5A1 & Down & E2F1 & 77 & PNCK \\
\hline Up & REST & 73 & BRI3 & Down & BMI1 & 70 & CBLN2 \\
\hline Up & SOX9 & 72 & CSRP2 & Down & FOXA2 & 70 & VSNL1 \\
\hline Up & MYCN & 72 & HIF1A & Down & FOXP1 & 69 & MAPK8IP3 \\
\hline Up & ATF3 & 71 & IL13RA2 & Down & YAP1 & 69 & SLC30A3 \\
\hline Up & TCF4 & 71 & KPNA2 & Down & TFAP2C & 66 & CNDP1 \\
\hline Up & EP300 & 70 & ACTG2 & Down & OLIG2 & 64 & RGS7BP \\
\hline Up & GATA1 & 67 & PITX1 & Down & ESR1 & 64 & S1PR5 \\
\hline Up & MYBL2 & 66 & WEE1 & Down & EP300 & 63 & FGFR3 \\
\hline Up & XRN2 & 64 & METTL7B & Down & SCLY & 60 & CCKBR \\
\hline Up & ESR1 & 63 & IGFBP3 & Down & SALL4 & 58 & CYP4X1 \\
\hline Up & ERG & 61 & DLGAP5 & Down & PPARG & 58 & INA \\
\hline Up & FOXO3 & 59 & GMNN & Down & $\mathrm{NR} 3 \mathrm{C} 1$ & 58 & JPH3 \\
\hline
\end{tabular}


medRxiv preprint doi: https://doi.org/10.1101/2020.12.21.20248616; this version posted December 23, 2020. The copyright holder for this preprint (which was not certified by peer review) is the author/funder, who has granted medRxiv a license to display the preprint in perpetuity. All rights reserved. No reuse allowed without permission.

\begin{tabular}{|c|c|c|c|c|c|c|c|}
\hline Up & TTF2 & 58 & $\mathrm{ADM}$ & Down & KDM5B & 57 & GABBR1 \\
\hline Up & SRY & 58 & APOC1 & Down & ZNF281 & 56 & FAIM2 \\
\hline Up & RELA & 58 & C1S & Down & TEAD4 & 51 & PPFIA2 \\
\hline Up & FOXA2 & 58 & CD99 & Down & SMAD3 & 48 & GABRB3 \\
\hline Up & SMAD2 & 58 & NUSAP1 & Down & ESRRB & 48 & SYN2 \\
\hline Up & CCND1 & 58 & ZBTB8OS & Down & JUN & 47 & NAV3 \\
\hline Up & SOX17 & 57 & TMSB10 & Down & TFAP2A & 46 & ASIC2 \\
\hline Up & RUNX2 & 54 & LAMC1 & Down & DMRT1 & 46 & ATP1B1 \\
\hline Up & TET1 & 53 & PTTG1 & Down & RAD21 & 46 & CREG2 \\
\hline Up & HOXB4 & 52 & RPLP1 & Down & BACH1 & 46 & OMG \\
\hline Up & WT1 & 52 & TFAP2A & Down & EOMES & 46 & RPRML \\
\hline Up & VDR & 51 & APH1A & Down & ASH2L & 45 & MAP1A \\
\hline Up & ZNF281 & 51 & SERPINH1 & Down & TCF7 & 44 & OGDHL \\
\hline Up & PRDM14 & 50 & RPL14 & Down & PBX1 & 43 & CNTNAP2 \\
\hline Up & ELK1 & 50 & RPS12 & Down & EED & 43 & KCNK12 \\
\hline Up & СЕВРВ & 49 & HMGB2 & Down & ERG & 43 & MYRIP \\
\hline Up & BACH1 & 49 & NSMAF & Down & FOXP2 & 42 & GRM3 \\
\hline Up & NFE2L2 & 48 & G3BP1 & Down & ZNF217 & 42 & KIF5C \\
\hline Up & NR0B1 & 48 & GBP1 & Down & TAL1 & 42 & PPP1R16B \\
\hline Up & TFCP2L1 & 48 & LY96 & Down & CTNNB1 & 41 & UGT8 \\
\hline Up & HOXC9 & 47 & FTH1 & Down & PAX3 & 39 & PEX5L \\
\hline Up & YAP1 & 47 & GPX8 & Down & WT1 & 38 & AGAP1 \\
\hline Up & MTF2 & 47 & ID2 & Down & E2F4 & 38 & $\mathrm{CH} 25 \mathrm{H}$ \\
\hline Up & TBX5 & 47 & PPIA & Down & POU3F2 & 38 & DNM3 \\
\hline Up & OLIG2 & 46 & RELL1 & Down & CUX1 & 38 & STMN2 \\
\hline Up & $\mathrm{NR} 3 \mathrm{C} 1$ & 45 & F3 & Down & YY1 & 38 & XIST \\
\hline Up & RCOR3 & 45 & HLA-A & Down & DROSHA & 36 & FAM189A1 \\
\hline Up & FOXP3 & 43 & ANXA1 & Down & TFCP2L1 & 36 & PAQR6 \\
\hline Up & DMRT1 & 43 & СMTM3 & Down & PRDM14 & 35 & CDS1 \\
\hline Up & SCLY & 43 & MAD2L1 & Down & EWSR1 & 34 & VIPR1 \\
\hline Up & JUN & 42 & LOX & Down & RCOR1 & 34 & VSTM2B \\
\hline
\end{tabular}


medRxiv preprint doi: https://doi.org/10.1101/2020.12.21.20248616; this version posted December 23, 2020. The copyright holder for this preprint (which was not certified by peer review) is the author/funder, who has granted medRxiv a license to display the preprint in perpetuity. All rights reserved. No reuse allowed without permission.

\begin{tabular}{|c|c|c|c|c|c|c|c|}
\hline Up & GFI1B & 42 & РPP2CB & Down & MYCN & 34 & ZFR2 \\
\hline Up & MECOM & 41 & TGIF1 & Down & TBX5 & 33 & KIAA0319 \\
\hline Up & FOXP2 & 40 & COL6A3 & Down & KLF1 & 33 & NRIP3 \\
\hline Up & PHF8 & 40 & STC2 & Down & NR0B1 & 32 & GAD2 \\
\hline Up & PBX1 & 39 & CDKN3 & Down & RBPJ & 32 & HSPA2 \\
\hline Up & FOXM1 & 39 & KIF11 & Down & PHC1 & 32 & NETO1 \\
\hline Up & GATA4 & 39 & RDH10 & Down & СЕВРВ & 31 & C1QTNF4 \\
\hline Up & ZNF217 & 38 & SOCS2 & Down & SIN3A & 31 & CHGA \\
\hline Up & ZFP42 & 37 & AURKA & Down & LMO2 & 29 & RASAL1 \\
\hline Up & RBPJ & 37 & PYGL & Down & TBX3 & 29 & SLC6A15 \\
\hline Up & BMI1 & 37 & RPS7 & Down & CTCF & 28 & NECAB2 \\
\hline Up & STAT4 & 36 & MPLKIP & Down & NFE2L2 & 27 & MAL \\
\hline $\mathrm{Up}$ & RAD21 & 36 & NEK2 & Down & $\mathrm{CDX} 2$ & 25 & GARNL3 \\
\hline Up & CNOT3 & 36 & PRPH & Down & ZFX & 25 & SH3GL2 \\
\hline Up & NUCKS1 & 35 & SOD2 & Down & DNAJC2 & 24 & CAMKV \\
\hline Up & EZH2 & 34 & PLAU & Down & NR1I2 & 23 & LHPP \\
\hline Up & KDM5A & 33 & NMB & Down & ARNT & 22 & $\mathrm{ERC} 2$ \\
\hline Up & DACH1 & 32 & MELK & Down & SOX11 & 22 & ST8SIA3 \\
\hline Up & CHD1 & 32 & RPS3 & Down & GFI1B & 22 & TUBB4A \\
\hline Up & RNF2 & 32 & SLC39A14 & Down & PAX6 & 21 & RALYL \\
\hline Up & STAT5A & 31 & LARP4 & Down & GATA4 & 20 & ARHGEF7 \\
\hline Up & POU3F2 & 31 & MMP7 & Down & MYBL2 & 20 & ТTC9B \\
\hline Up & IRF8 & 31 & TPT1 & Down & SOX17 & 19 & BHLHE22 \\
\hline Up & RCOR1 & 31 & VCAM1 & Down & RCOR2 & 19 & DLGAP2 \\
\hline Up & SIN3A & 30 & TNFRSF19 & Down & ELK1 & 19 & OLFM1 \\
\hline Up & TEAD4 & 29 & STC1 & Down & ELF5 & 19 & RIMBP2 \\
\hline Up & MEF2A & 28 & ASPM & Down & STAT1 & 19 & SLC1A2 \\
\hline Up & NR1H3 & 28 & CD93 & Down & FOXO3 & 19 & STXBP6 \\
\hline Up & ESRRB & 28 & HOXA5 & Down & HOXC9 & 19 & TEF \\
\hline Up & MYB & 28 & RAP1B & Down & SREBF2 & 19 & TMEM151A \\
\hline Up & BCL3 & 27 & FAM162A & Down & SREBF1 & 18 & ANKRD24 \\
\hline
\end{tabular}


medRxiv preprint doi: https://doi.org/10.1101/2020.12.21.20248616; this version posted December 23, 2020. The copyright holder for this preprint (which was not certified by peer review) is the author/funder, who has granted medRxiv a license to display the preprint in perpetuity. All rights reserved. No reuse allowed without permission.

\begin{tabular}{|c|c|c|c|c|c|c|c|}
\hline Up & PRDM5 & 26 & CLIC1 & Down & LYL1 & 18 & CLDN10 \\
\hline Up & EWSR1 & 26 & PAPSS1 & Down & MEIS1 & 18 & STX1B \\
\hline Up & $\mathrm{CDX} 2$ & 25 & GGH & Down & TCF7L2 & 17 & ELAVL3 \\
\hline Up & TBP & 25 & PLEKHA4 & Down & SMAD2 & 17 & KLK6 \\
\hline Up & SRF & 24 & USP8 & Down & AHR & 17 & MIAT \\
\hline Up & LMO2 & 24 & IGFBP4 & Down & GATA3 & 17 & PTPRT \\
\hline Up & HSF1 & 24 & RPS13 & Down & CCND1 & 17 & SRRM4 \\
\hline Up & CTNNB1 & 24 & STEAP3 & Down & RELA & 16 & GRIN1 \\
\hline Up & MEIS1 & 23 & IER3IP1 & Down & NACC1 & 16 & MAP7 \\
\hline Up & PADI4 & 23 & NEDD9 & Down & MECOM & 16 & PAQR8 \\
\hline Up & AP1S2 & 23 & RPS29 & Down & ZFP42 & 15 & GNG3 \\
\hline Up & ASXL1 & 23 & TUBB6 & Down & ATF3 & 15 & ITPKA \\
\hline Up & TFEB & 23 & WLS & Down & HTT & 15 & MAG \\
\hline Up & TAF7L & 22 & DBI & Down & CNOT3 & 15 & NKX6-2 \\
\hline Up & SOX11 & 22 & HSPE1 & Down & ESR2 & 14 & ARRB1 \\
\hline Up & CTCF & 22 & NNMT & Down & IRF8 & 14 & ASPA \\
\hline Up & PAX3 & 21 & BCHE & Down & GBX2 & 14 & CCDC85A \\
\hline Up & PDX1 & 21 & C1QTNF1 & Down & HSF1 & 14 & CELF2 \\
\hline Up & $\mathrm{PHC} 1$ & 21 & $\mathrm{CDC} 45$ & Down & MEF2A & 14 & KCNH3 \\
\hline Up & RARG & 21 & COL1A1 & Down & NFIB & 13 & CDK5R1 \\
\hline Up & NACC1 & 21 & IGFBP2 & Down & DACH1 & 13 & PIP4K2A \\
\hline Up & ELF1 & 20 & ACTR3 & Down & STAT4 & 13 & PPP1R3F \\
\hline Up & JARID2 & 19 & LYPD1 & Down & PDX1 & 12 & AMPH \\
\hline Up & PAX6 & 19 & RPL7A & Down & NR4A2 & 12 & STMN4 \\
\hline Up & GATA3 & 18 & LGALS3 & Down & STAT5A & 11 & STOX2 \\
\hline Up & CLOCK & 18 & SP3 & Down & ELF1 & 11 & FOSB \\
\hline Up & CEBPA & 17 & CYP51A1 & Down & MYB & 11 & PKP4 \\
\hline Up & TBX3 & 17 & GNG10 & Down & TTF2 & 10 & APLP1 \\
\hline Up & ELF5 & 17 & HSPA4 & Down & XRN2 & 10 & ERBB3 \\
\hline Up & EED & 16 & CAV1 & Down & ETS1 & 10 & SYT13 \\
\hline Up & TCF7L2 & 16 & MTHFD2 & Down & CEBPD & 10 & TMEFF2 \\
\hline
\end{tabular}


medRxiv preprint doi: https://doi.org/10.1101/2020.12.21.20248616; this version posted December 23, 2020. The copyright holder for this preprint (which was not certified by peer review) is the author/funder, who has granted medRxiv a license to display the preprint in perpetuity. All rights reserved. No reuse allowed without permission.

\begin{tabular}{|c|c|c|c|c|c|c|c|}
\hline Up & ARNT & 16 & S100A4 & Down & THAP11 & 9 & CA11 \\
\hline Up & SREBF1 & 15 & RPS28 & Down & VDR & 9 & CCL4L1 \\
\hline Up & SREBF2 & 15 & TSPAN12 & Down & CRX & 9 & CELF3 \\
\hline Up & ZIC3 & 14 & PDLIM1 & Down & PHF8 & 9 & NPTX1 \\
\hline Up & ESR2 & 14 & PLOD2 & Down & SMAD1 & 9 & RAB6B \\
\hline Up & DCP1A & 14 & SEC61G & Down & IKZF1 & 8 & CAMK2A \\
\hline Up & IRF1 & 13 & CLK1 & Down & TAF7L & 8 & DHRS9 \\
\hline Up & SMAD1 & 13 & EEF1B2 & Down & NUCKS1 & 8 & KLC1 \\
\hline Up & NR1I2 & 13 & HP & Down & THRA & 8 & PDE2A \\
\hline Up & AHR & 12 & ADAMTS9 & Down & CLOCK & 7 & BSN \\
\hline Up & LYL1 & 12 & CHI3L1 & Down & HOXB4 & 7 & FSTL5 \\
\hline Up & NFIB & 12 & RPL6 & Down & STAT6 & 7 & MAST3 \\
\hline Up & KDM6A & 11 & CNBP & Down & NR1H3 & 7 & MBP \\
\hline Up & HTT & 10 & CCNB1 & Down & SRF & 7 & NAPB \\
\hline Up & DNAJC2 & 10 & LGALS1 & Down & KDM5A & 7 & RAB40B \\
\hline Up & TCF7 & 10 & SUMO2 & Down & TBP & 6 & DLG4 \\
\hline Up & THAP11 & 9 & CNIH4 & Down & TFEB & 6 & KIAA0513 \\
\hline Up & CRX & 9 & PTMA & Down & ZIC3 & 6 & MAP4 \\
\hline Up & CEBPD & 9 & TNC & Down & BCL3 & 6 & NCS1 \\
\hline Up & HCFC1 & 8 & CCT8 & Down & FOXO1 & 6 & NPY \\
\hline Up & STAT1 & 8 & RPL15 & Down & FOXP3 & 6 & PARM1 \\
\hline Up & STAT6 & 7 & CD44 & Down & CDKN2AIP & 5 & CALY \\
\hline Up & HOXD13 & 6 & MXRA5 & Down & RARG & 5 & DNM1 \\
\hline Up & RCOR2 & 6 & VIM & Down & IRF1 & 5 & FBXL16 \\
\hline $\mathrm{Up}$ & FOXO1 & 6 & ZFP36L1 & Down & TCF21 & 5 & IL1RAPL1 \\
\hline Up & MYBL1 & 5 & KIF20A & Down & ASXL1 & 5 & PPM1H \\
\hline Up & GBX2 & 5 & P4HA1 & Down & AP1S2 & 5 & SLC17A7 \\
\hline Up & CHD7 & 4 & EEF1A1 & Down & ETS2 & 5 & SYT7 \\
\hline Up & KLF2 & 4 & HOXC6 & Down & CEBPA & 5 & $\mathrm{TF}$ \\
\hline Up & E2F7 & 4 & PCNA & Down & PADI4 & 5 & TMEM125 \\
\hline Up & NOTCH1 & 4 & RPL18A & Down & ZNF652 & 4 & LAMP5 \\
\hline
\end{tabular}


medRxiv preprint doi: https://doi.org/10.1101/2020.12.21.20248616; this version posted December 23, 2020. The copyright holder for this preprint (which was not certified by peer review) is the author/funder, who has granted medRxiv a license to display the preprint in perpetuity. All rights reserved. No reuse allowed without permission.

\begin{tabular}{|c|c|c|c|c|c|c|c|}
\hline $\mathrm{Up}$ & KLF5 & 4 & SERPINA3 & Down & PRDM5 & 4 & MAL2 \\
\hline $\mathrm{Up}$ & SALL1 & 3 & ECT2 & Down & CHD1 & 4 & SYNGR1 \\
\hline $\mathrm{Up}$ & TCF21 & 3 & IL1RAP & Down & PRDM16 & 3 & CAMK1D \\
\hline Up & IKZF1 & 3 & GPX7 & Down & DCP1A & 3 & $\mathrm{CNP}$ \\
\hline Up & NR4A2 & 3 & PRCP & Down & ZNF322 & 3 & KCTD16 \\
\hline Up & ETS2 & 3 & TAGLN2 & Down & HOXD13 & 3 & NRSN1 \\
\hline $\mathrm{Up}$ & ZNF274 & 3 & TPX2 & Down & ZNF274 & 3 & PEG3 \\
\hline Up & DROSHA & 2 & $\mathrm{AK} 4$ & Down & GLI1 & 3 & PLEKHB1 \\
\hline Up & ZNF263 & 1 & GDF15 & Down & HIF1A & 2 & RAP1GAP2 \\
\hline Up & HOXA2 & 1 & HMMR & Down & KDM6A & 2 & SERPINI1 \\
\hline Up & BCL11B & 1 & ID3 & Down & NOTCH1 & 2 & SOWAHA \\
\hline Up & THRA & 1 & SMS & Down & ZNF263 & 1 & ITPKA \\
\hline $\mathrm{Up}$ & PRDM16 & 1 & TSC22D1 & Down & SALL1 & 1 & RTN1 \\
\hline Up & CIITA & 1 & KDM3A & & & & \\
\hline Up & BP1 & 1 & VEGFA & & & & \\
\hline
\end{tabular}

Degree - No of TF interact with target gene. We taken any one TF in table

Table 9. Primers used for quantitative PCR

Primer sequence $\left(5^{\prime} \rightarrow 3^{\prime}\right)$

$\begin{array}{ccc}\text { TUBA1C } & \text { TGTTTGTAGACTTGGAACCCAC } & \text { GCCAATGGTGTAGTGCCCT } \\ \text { CAV1 } & \text { GCGACCCTAAACACCTCAAC } & \text { ATGCCGTCAAAACTGTGTGTC } \\ \text { RPL23 } & \text { TCCTCTGGTGCGAAATTCCG } & \text { CGTCCCTTGATCCCCTTCAC } \\ \text { YY1 } & \text { ACGGCTTCGAGGATCAGATTC } & \text { TGACCAGCGTTTGTTCAATGT } \\ \text { S100A4 } & \text { GATGAGCAACTTGGACAGCAA } & \text { CTGGGCTGCTTATCTGGGAAG } \\ \text { ARHGEF7 } & \text { TGCTTTCAACGTACCTACGGC } & \text { GGCAACTTGGTGCATTCTTCTAA } \\ \text { DNAJA4 } & \text { GGGATGTTTATGACCAAGGCG } & \text { GCCAATTTCTTCGTGACTCCA } \\ \text { PAK6 } & \text { ACCAATAGGCATGGAATGAAGG } & \text { GCGGTCGGAAAGAGGAGTTG } \\ \text { NELL1 } & \text { TTTGGGATGGACCCTGACCTT } & \text { CATTGTGCATTCCAGACACCT }\end{array}$


ITPKA

\section{Figures}

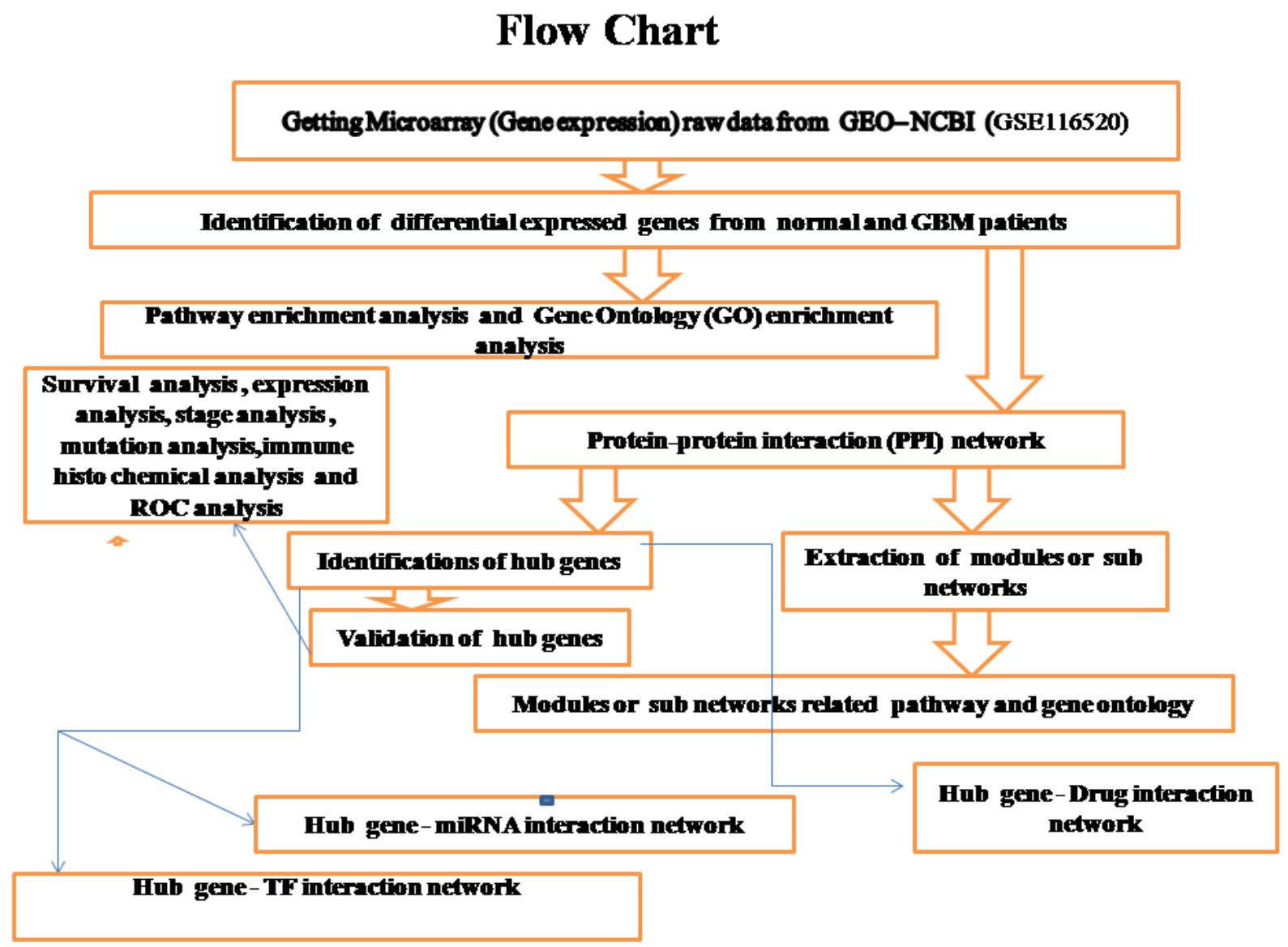

Fig. 1.Study design (flow diagram of study) 
medRxiv preprint doi: https://doi.org/10.1101/2020.12.21.20248616; this version posted December 23, 2020. The copyright holder for this preprint (which was not certified by peer review) is the author/funder, who has granted medRxiv a license to display the preprint in perpetuity.

All rights reserved. No reuse allowed without permission.
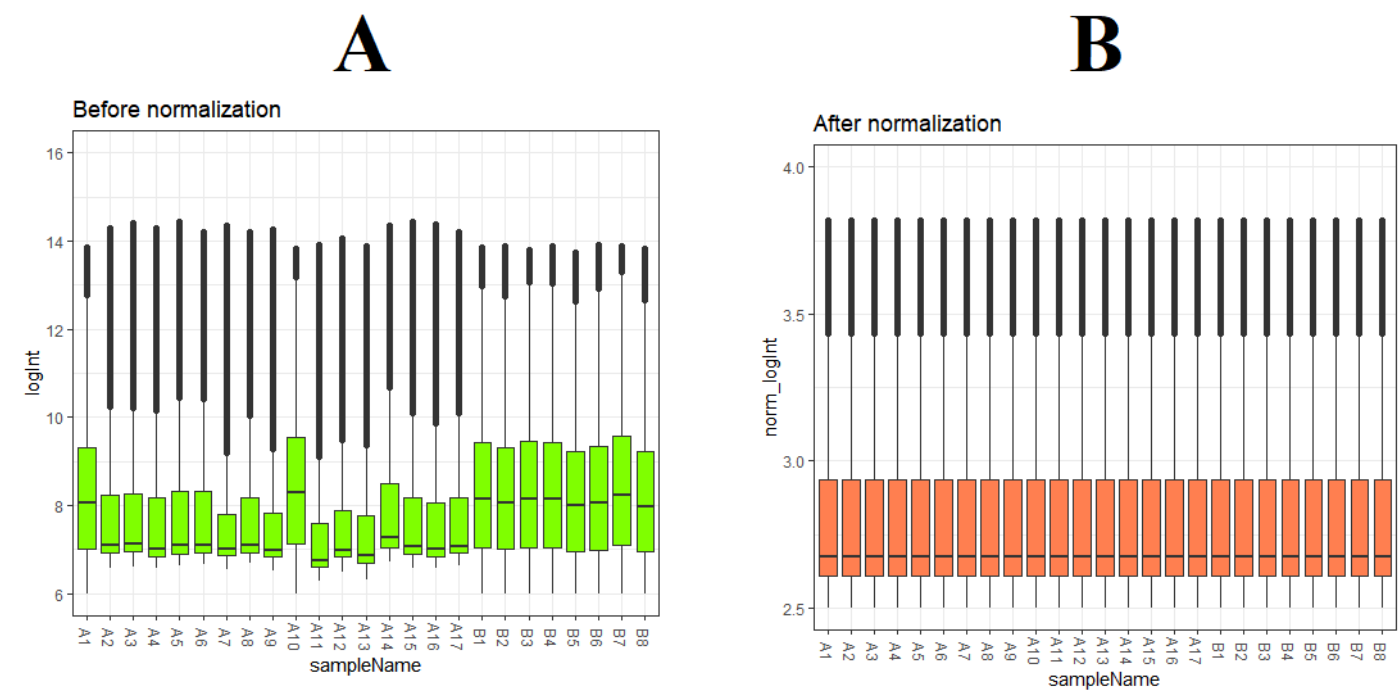

Fig. 2. Box plots of the gene expression data before normalization (A) and after normalization (B). Horizontal axis represents the sample symbol and the vertical axis represents the gene expression values. The black line in the box plot represents the median value of gene expression. (A1 - A17 = GBM tissues samples; B1 - B8 = normal control samples) 
medRxiv preprint doi: https://doi.org/10.1101/2020.12.21.20248616; this version posted December 23, 2020. The copyright holder for this preprint (which was not certified by peer review) is the author/funder, who has granted medRxiv a license to display the preprint in perpetuity.

All rights reserved. No reuse allowed without permission.

\section{Volcano plot}

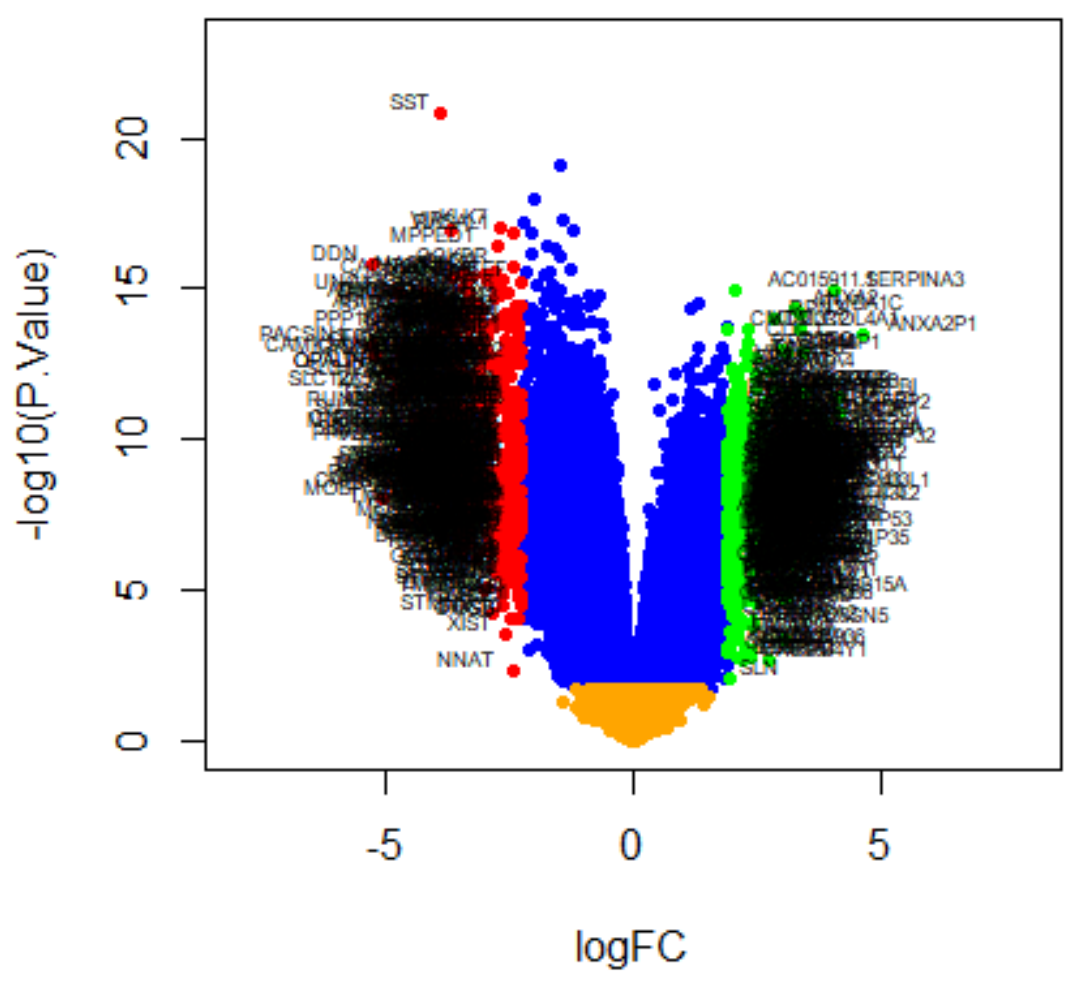

Fig. 3. Volcano plot of differentially expressed genes. Genes with a significant change of more than two-fold were selected. 
medRxiv preprint doi: https://doi.org/10.1101/2020.12.21.20248616; this version posted December 23, 2020. The copyright holder for this preprint (which was not certified by peer review) is the author/funder, who has granted medRxiv a license to display the preprint in perpetuity.

All rights reserved. No reuse allowed without permission.

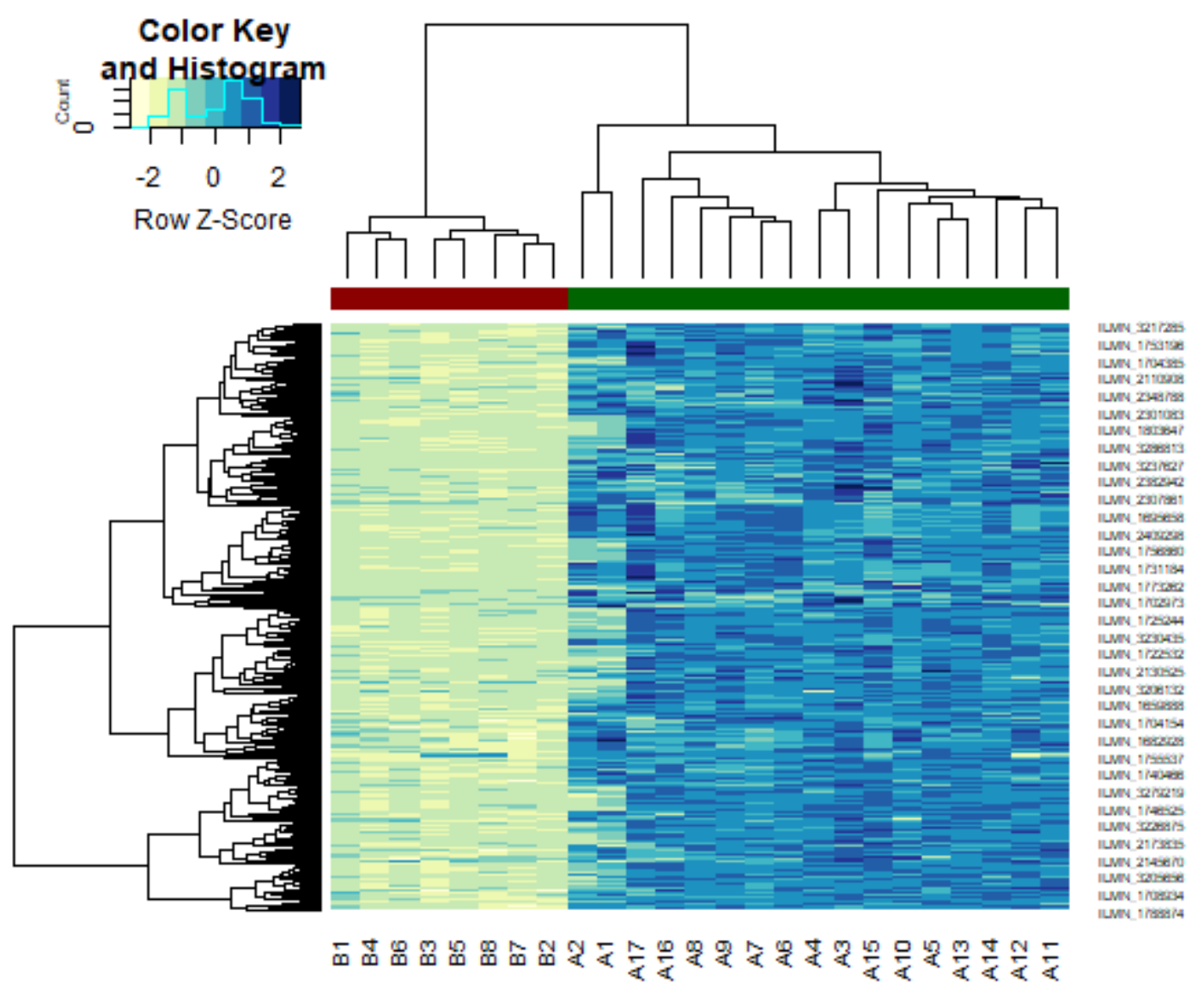

Fig. 4. Heat map of up regulated differentially expressed genes. Legend on the top left indicate log fold change of genes. $(\mathrm{A} 1-\mathrm{A} 17=\mathrm{GBM}$ tissues samples; $\mathrm{B} 1-\mathrm{B} 8=$ normal control samples $)$ 
medRxiv preprint doi: https://doi.org/10.1101/2020.12.21.20248616; this version posted December 23, 2020. The copyright holder for this preprint (which was not certified by peer review) is the author/funder, who has granted medRxiv a license to display the preprint in perpetuity.

All rights reserved. No reuse allowed without permission.
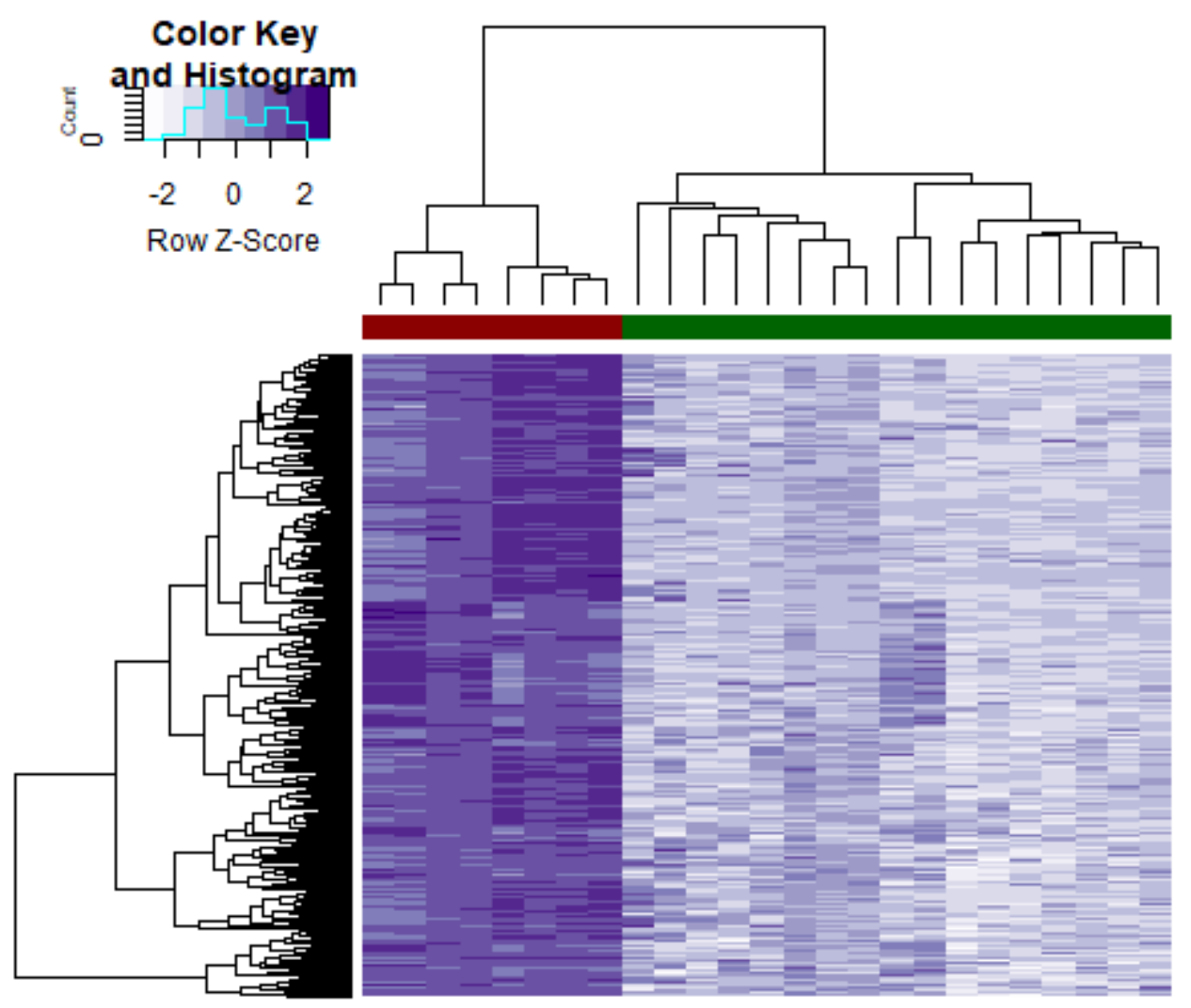

IUM⿻ irstex IUNA 200002 IUMV Tsing IISTN

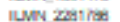
IUMv IUNe ineses

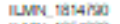
IUWN resercs IUMN Inteini? IUNN 20003/2 IUMN INarsin IUMN resosis? IUMN tratses lump ierions lomi semat

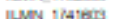
IUNN Ienesas ILMN irsaras? IUMve 2tons.

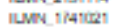
IUMv testres: IUMV tansis ILMN Troess IUMN Irsesed IUMN IUMN IITSA

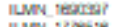
lumv tricsite IIMUN irsersen IUMN . T/A The

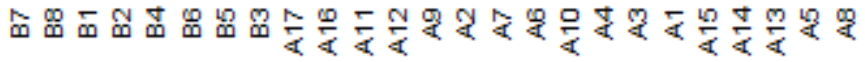

Fig. 5. Heat map of down regulated differentially expressed genes. Legend on the top left indicate log fold change of genes. $(\mathrm{A} 1-\mathrm{A} 17=\mathrm{GBM}$ tissues samples; $\mathrm{B} 1-\mathrm{B} 8=$ normal control samples $)$
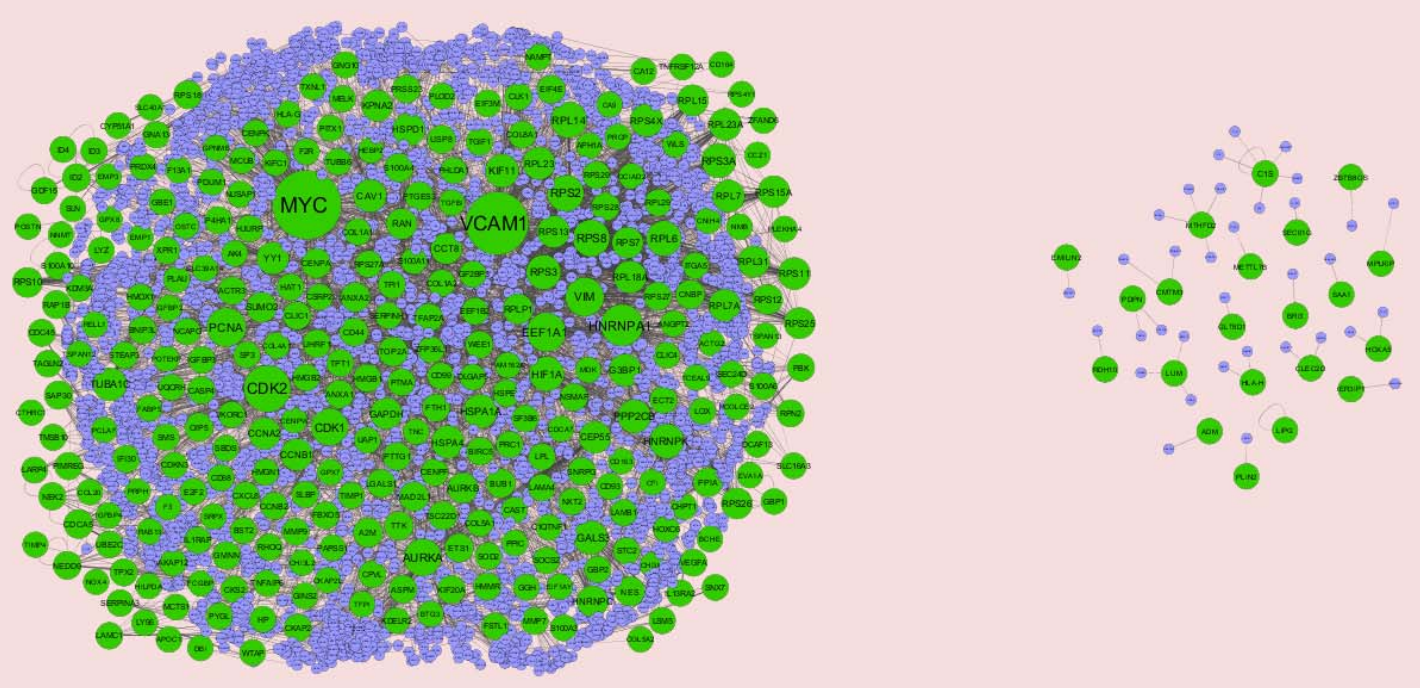

Fig. 6. Protein-protein interaction network of differentially expressed genes (DEGs). Green nodes denotes up regulated genes. 
medRxiv preprint doi: https://doi.org/10.1101/2020.12.21.20248616; this version posted December 23, 2020. The copyright holder for this preprint (which was not certified by peer review) is the author/funder, who has granted medRxiv a license to display the preprint in perpetuity.

All rights reserved. No reuse allowed without permission.

A

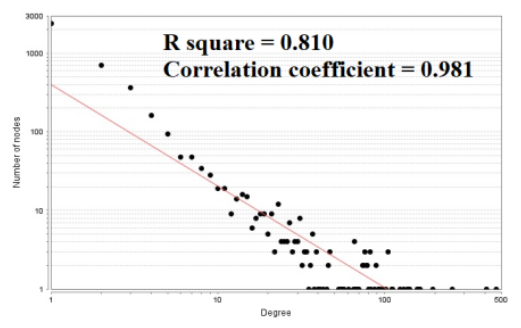

D

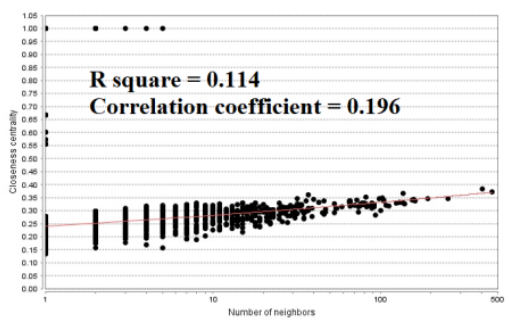

B
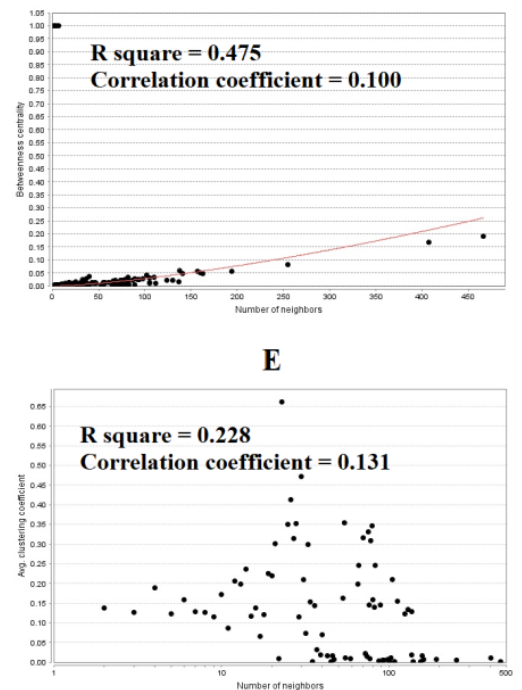

C

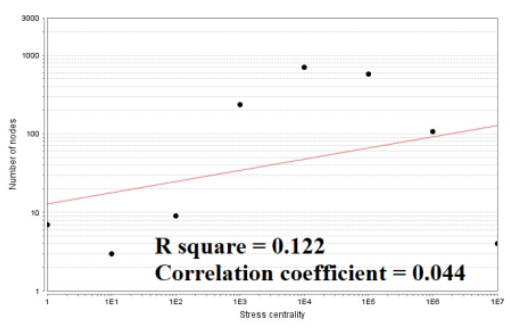

R square $=\mathbf{0 . 1 2 2}$

isterss cemalate

Fig. 7. Scatter plot for up regulated genes. (A- Node degree; B- Betweenness centrality; C- Stress centrality ; DCloseness centrality; E- Clustering coefficient)
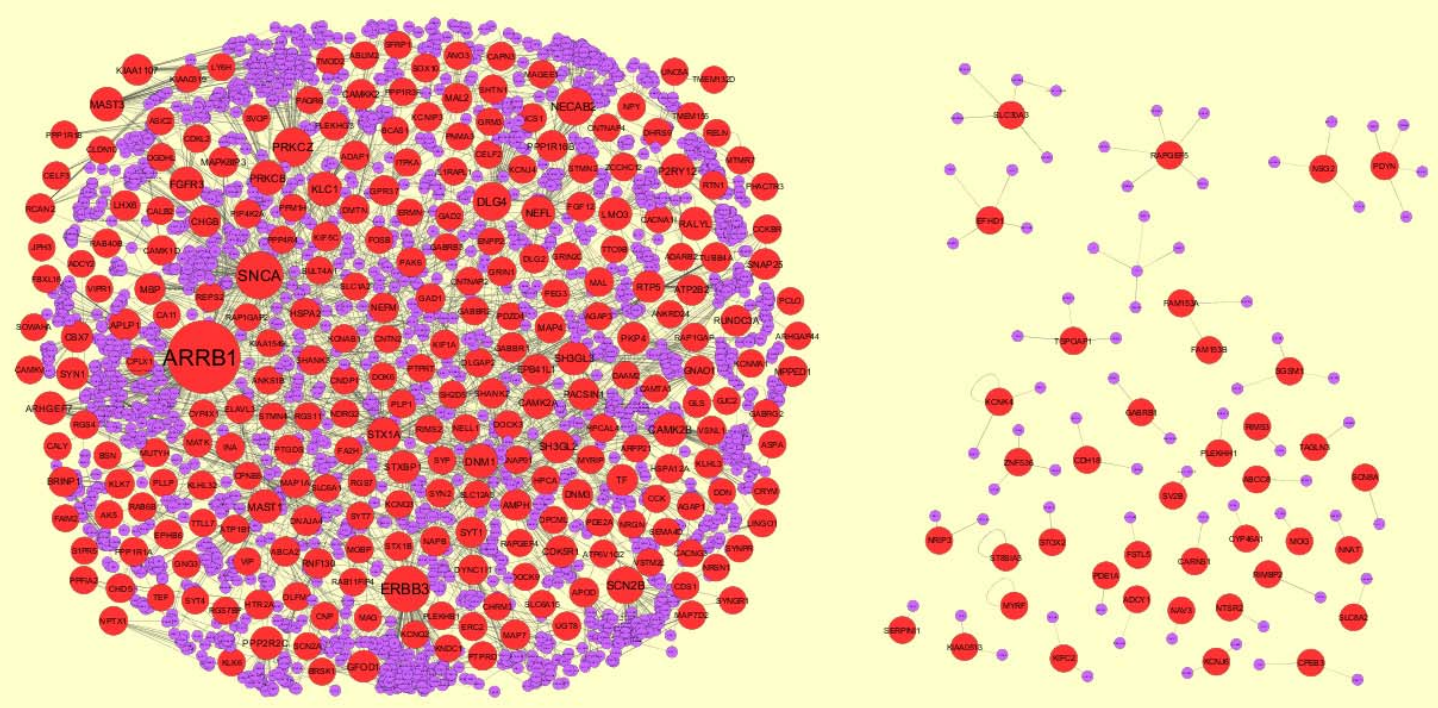

Fig. 8. Protein-protein interaction network of differentially expressed genes (DEGs). Red nodes denotes down regulated genes. 
medRxiv preprint doi: https://doi.org/10.1101/2020.12.21.20248616; this version posted December 23, 2020. The copyright holder for this preprint (which was not certified by peer review) is the author/funder, who has granted medRxiv a license to display the preprint in perpetuity.

All rights reserved. No reuse allowed without permission.

A

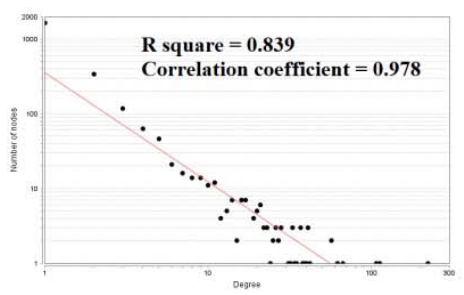

D

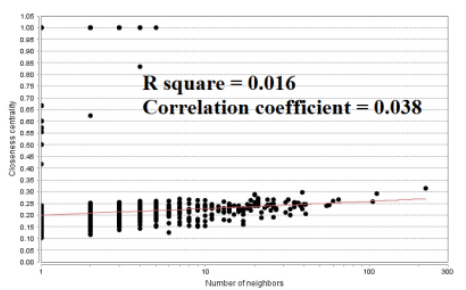

B

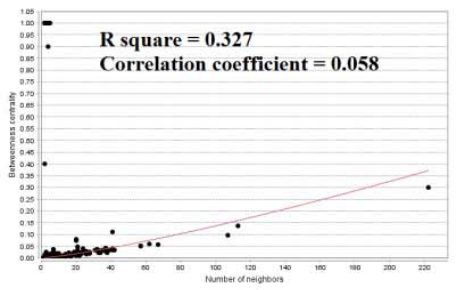

$\mathbf{E}$

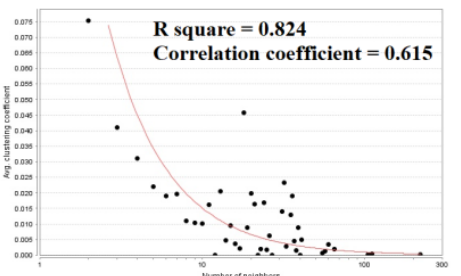

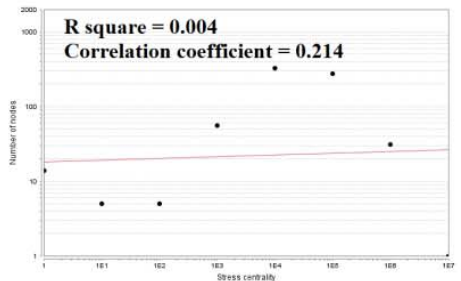

Fig. 9. Scatter plot for down regulated genes. (A- Node degree; B- Betweenness centrality; C- Stress centrality ; DCloseness centrality; E- Clustering coefficient)

Module 6

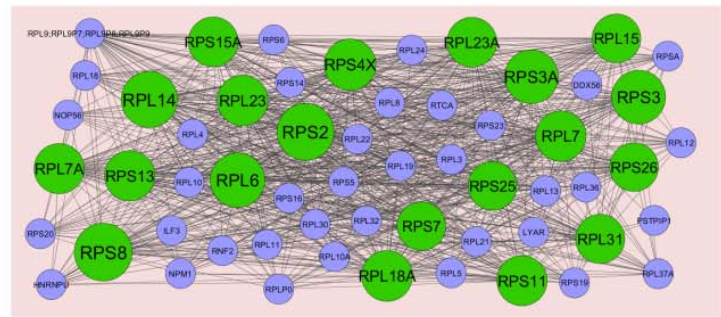

Module 24

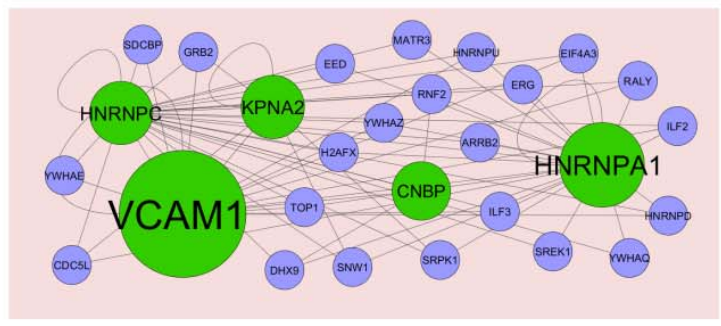

Module 15

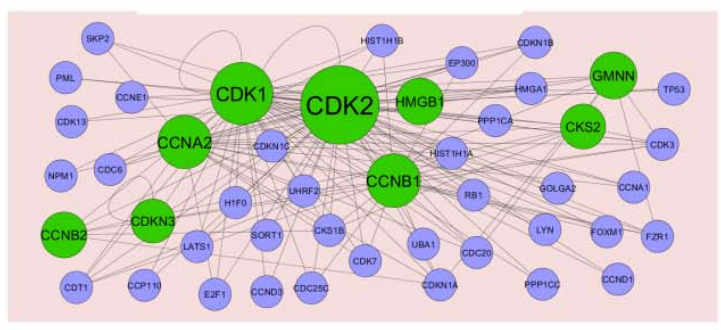

Module 51

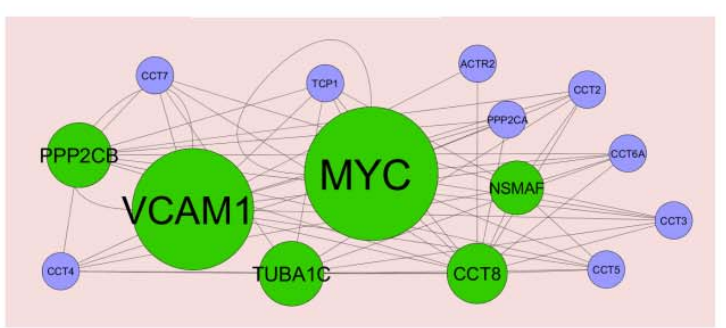

Fig. 10. Modules in PPI network. The green nodes denote the up regulated genes 
medRxiv preprint doi: https://doi.org/10.1101/2020.12.21.20248616; this version posted December 23, 2020. The copyright holder for this preprint (which was not certified by peer review) is the author/funder, who has granted medRxiv a license to display the preprint in perpetuity. All rights reserved. No reuse allowed without permission.

Module 2

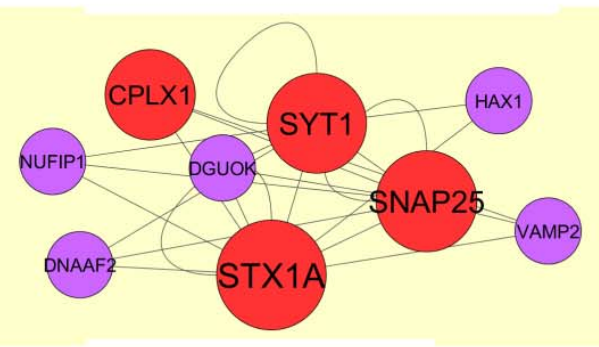

Module 18

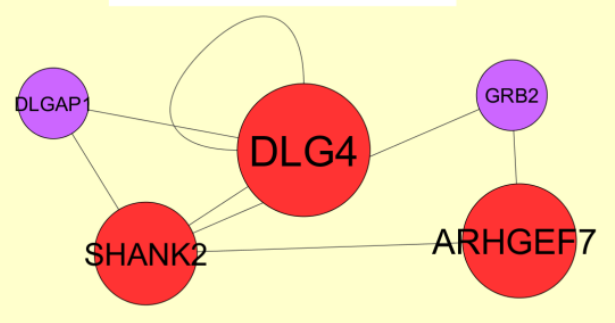

Module 7

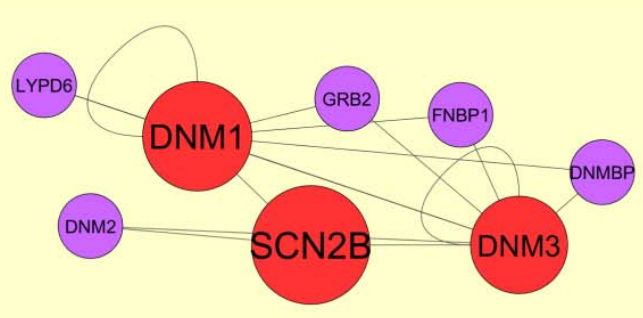

Module 22

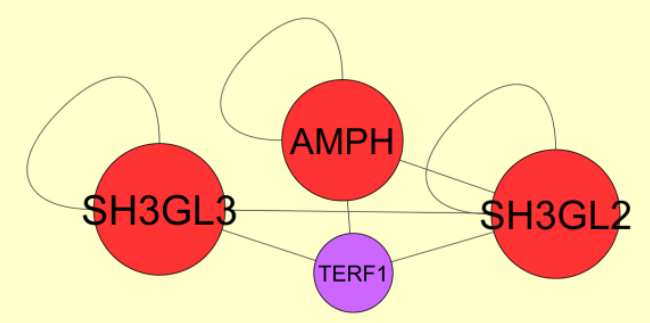

Fig. 11. Modules in PPI network. The red nodes denote the down regulated genes

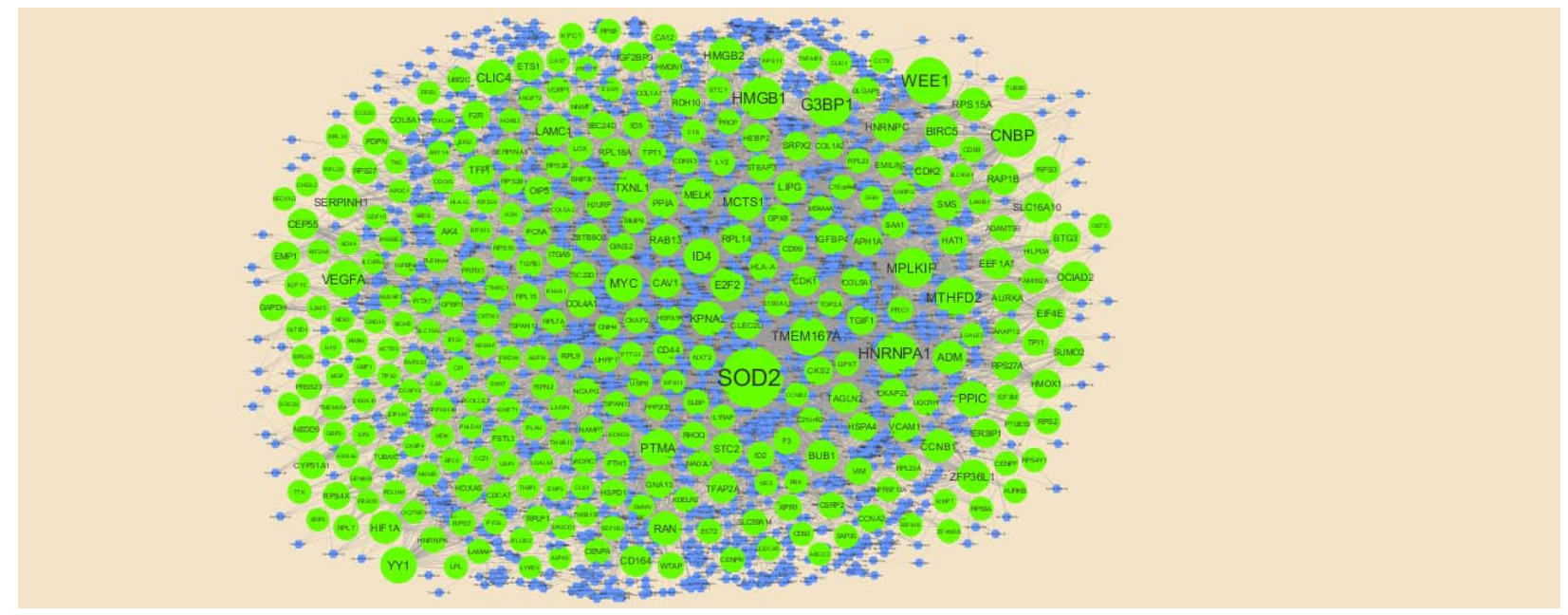

Fig. 12. The network of up regulated genes and their related miRNAs. The green circles nodes are the up regulated genes, and blue diamond nodes are the miRNAs 


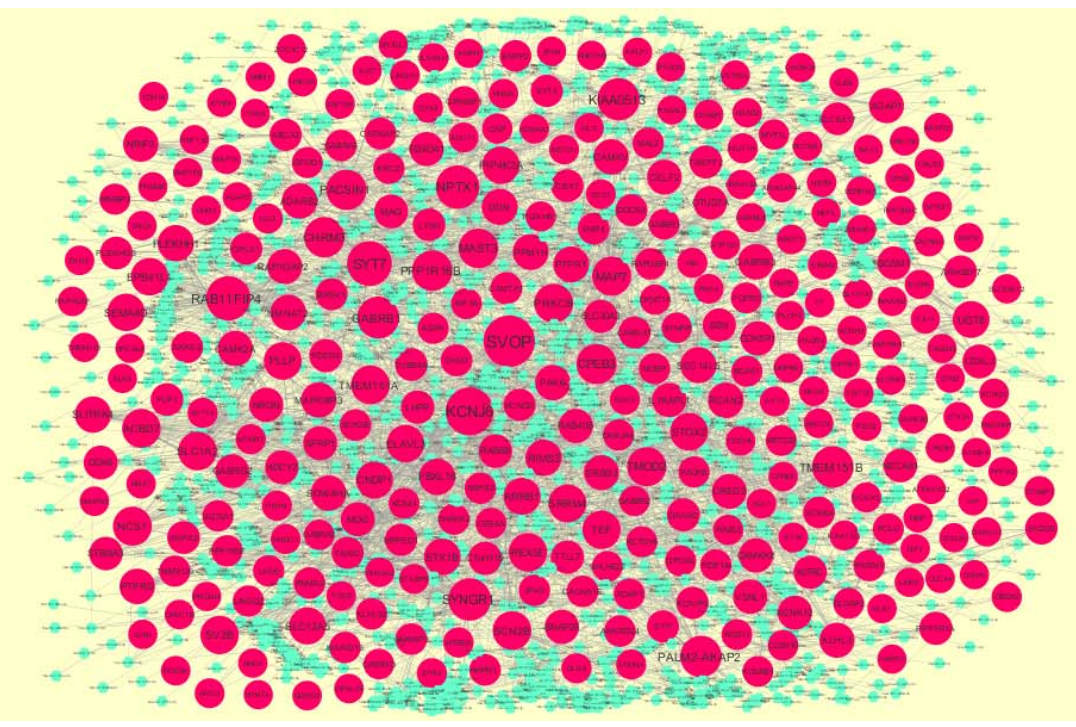

Fig. 13. The network of down regulated genes and their related miRNAs. The pink circles nodes are the down regulated genes, and sky blue diamond nodes are the miRNAs

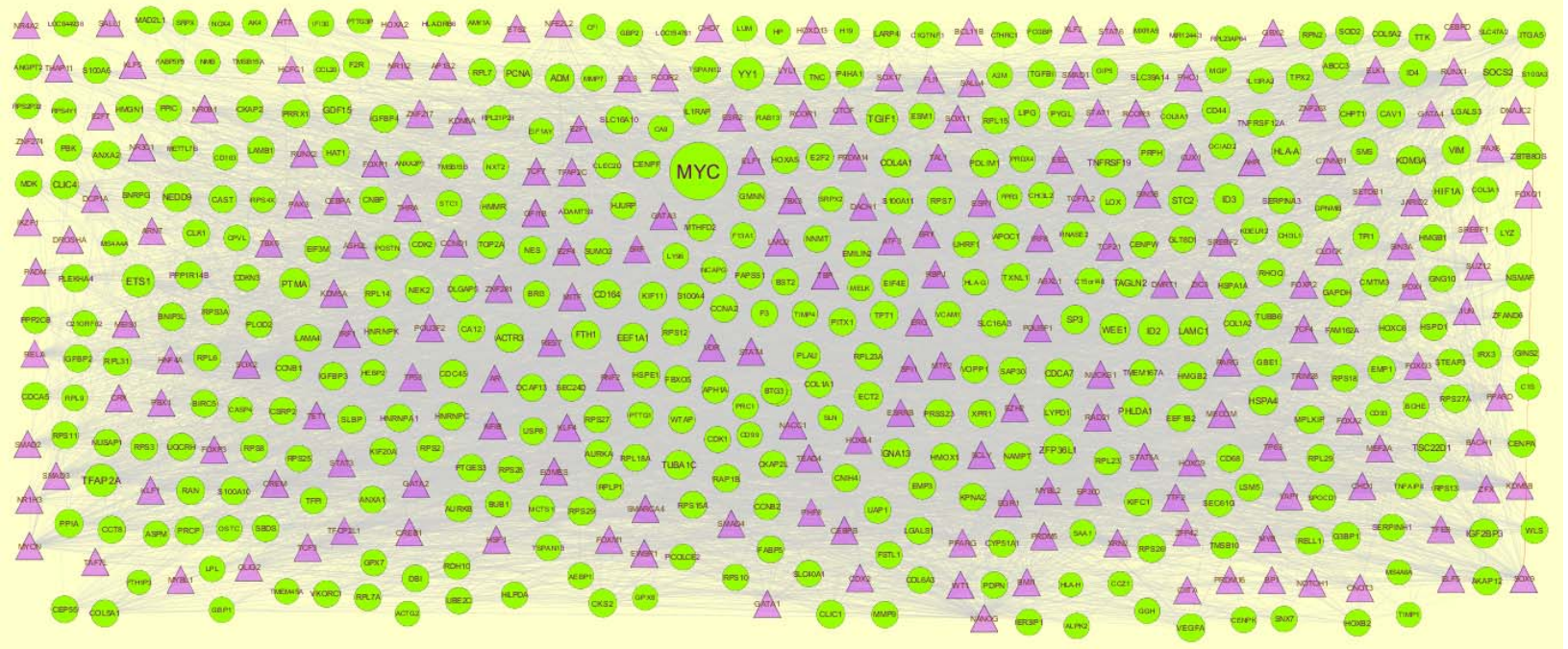

Fig. 14. The network of up regulated genes and their related TFs. The green circles nodes are the up regulated genes, and purple triangle nodes are the TFs 
medRxiv preprint doi: https://doi.org/10.1101/2020.12.21.20248616; this version posted December 23, 2020. The copyright holder for this preprint (which was not certified by peer review) is the author/funder, who has granted medRxiv a license to display the preprint in perpetuity. All rights reserved. No reuse allowed without permission.

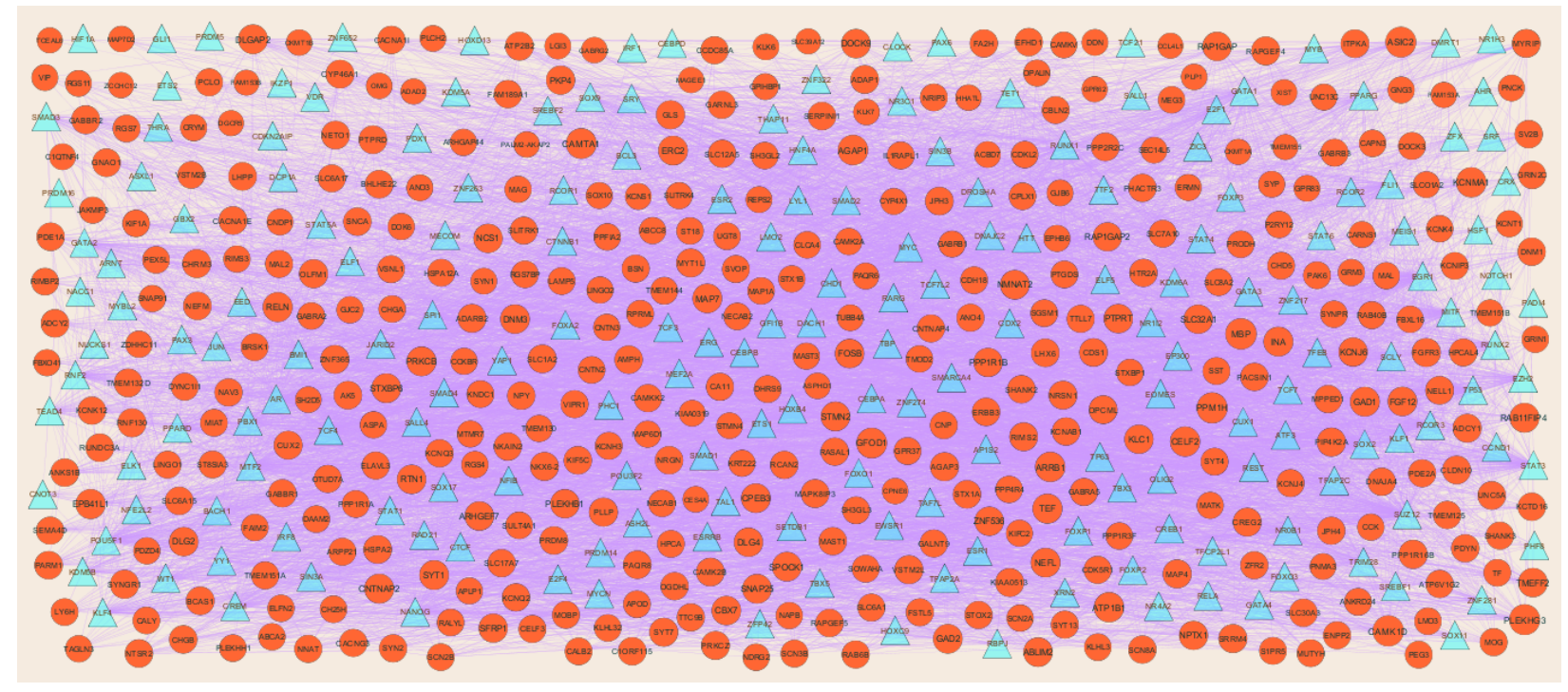

Fig. 15. The network of down regulated genes and their related TFs. The green circles nodes are the down regulated genes, and blue triangle nodes are the TFs
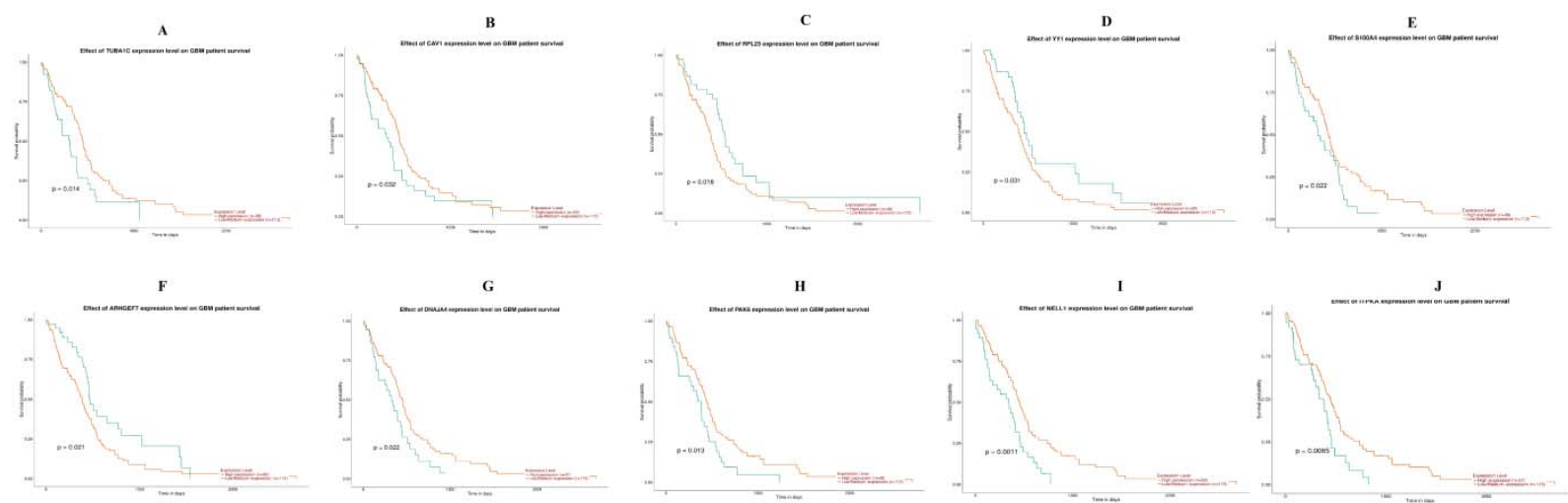

Fig. 16. Overall survival analysis of hub genes. Overall survival analyses were performed using the UALCAN online platform A) TUBA1C B) CAV1 C) RPL23 D) YY1 E) S100A4 F) ARHGEF7 G) DNAJA4 H) PAK6 I) NELL1 J) ITPKA 
medRxiv preprint doi: https://doi.org/10.1101/2020.12.21.20248616; this version posted December 23, 2020. The copyright holder for this preprint (which was not certified by peer review) is the author/funder, who has granted medRxiv a license to display the preprint in perpetuity. All rights reserved. No reuse allowed without permission.
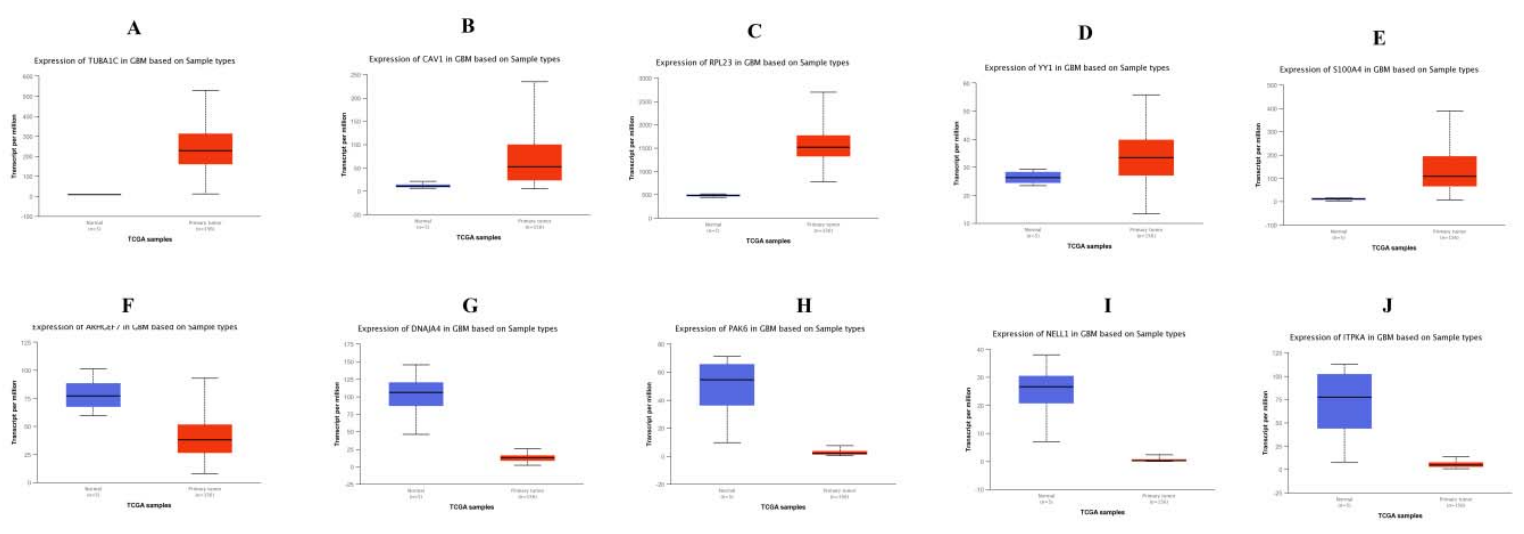

Fig. 17. Box plots (expression analysis) hub genes were produced using the UALCAN platform A) TUBA1C B) CAV1 C) RPL23 D) YY1 E) S100A4 F) ARHGEF7 G) DNAJA4 H) PAK6 I) NELL1 J) ITPKA

A

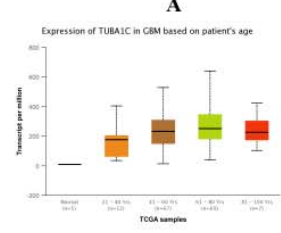

F

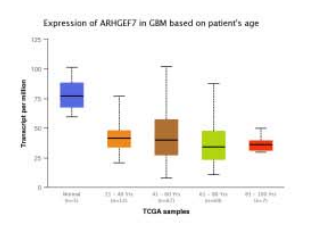

B
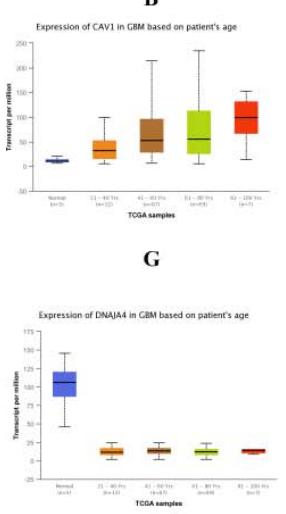

C

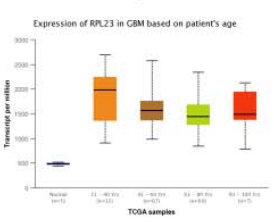

H

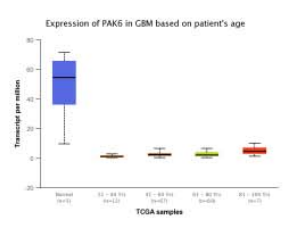

D

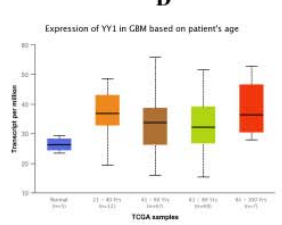

I

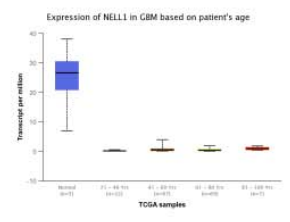

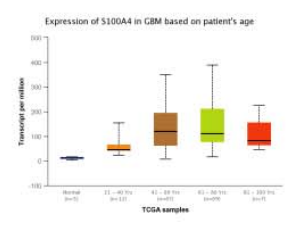

J

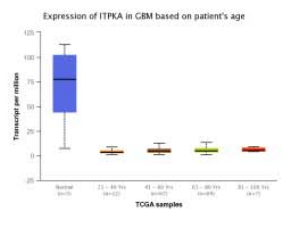

Fig. 18. Box plots (age analysis) of hub genes were produced using the UALCAN platform A) TUBA1C B) CAV1 C) RPL23 D) YY1 E) S100A4 F) ARHGEF7 G) DNAJA4 H) PAK6 I) NELL1 J) ITPKA 
medRxiv preprint doi: https://doi.org/10.1101/2020.12.21.20248616; this version posted December 23, 2020. The copyright holder for this preprint (which was not certified by peer review) is the author/funder, who has granted medRxiv a license to display the preprint in perpetuity.

All rights reserved. No reuse allowed without permission.

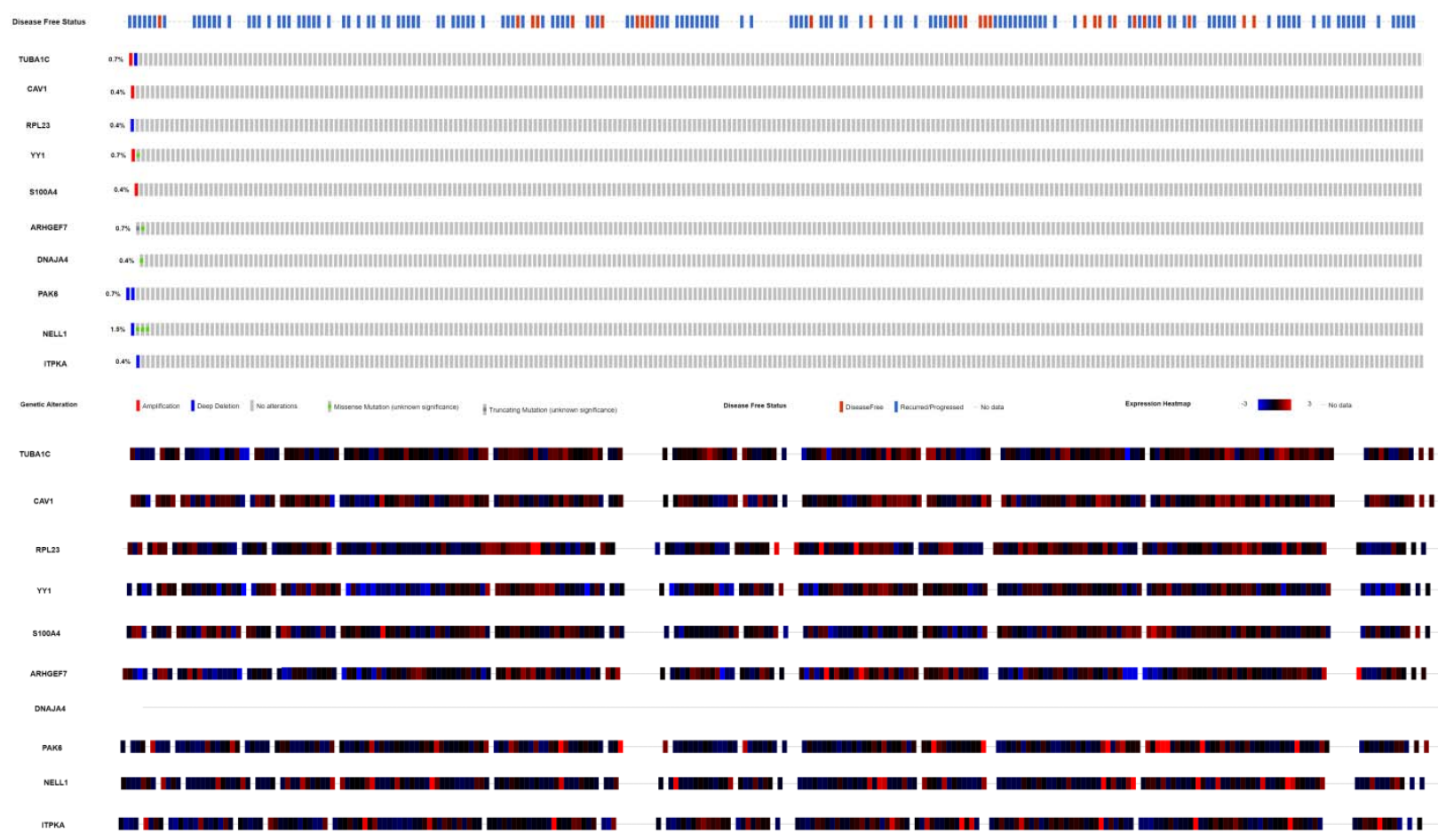

Fig. 19. Mutation analyses of hub genes were produced using the CbioPortal online platform 
medRxiv preprint doi: https://doi.org/10.1101/2020.12.21.20248616; this version posted December 23, 2020. The copyright holder for this preprint (which was not certified by peer review) is the author/funder, who has granted medRxiv a license to display the preprint in perpetuity. All rights reserved. No reuse allowed without permission.

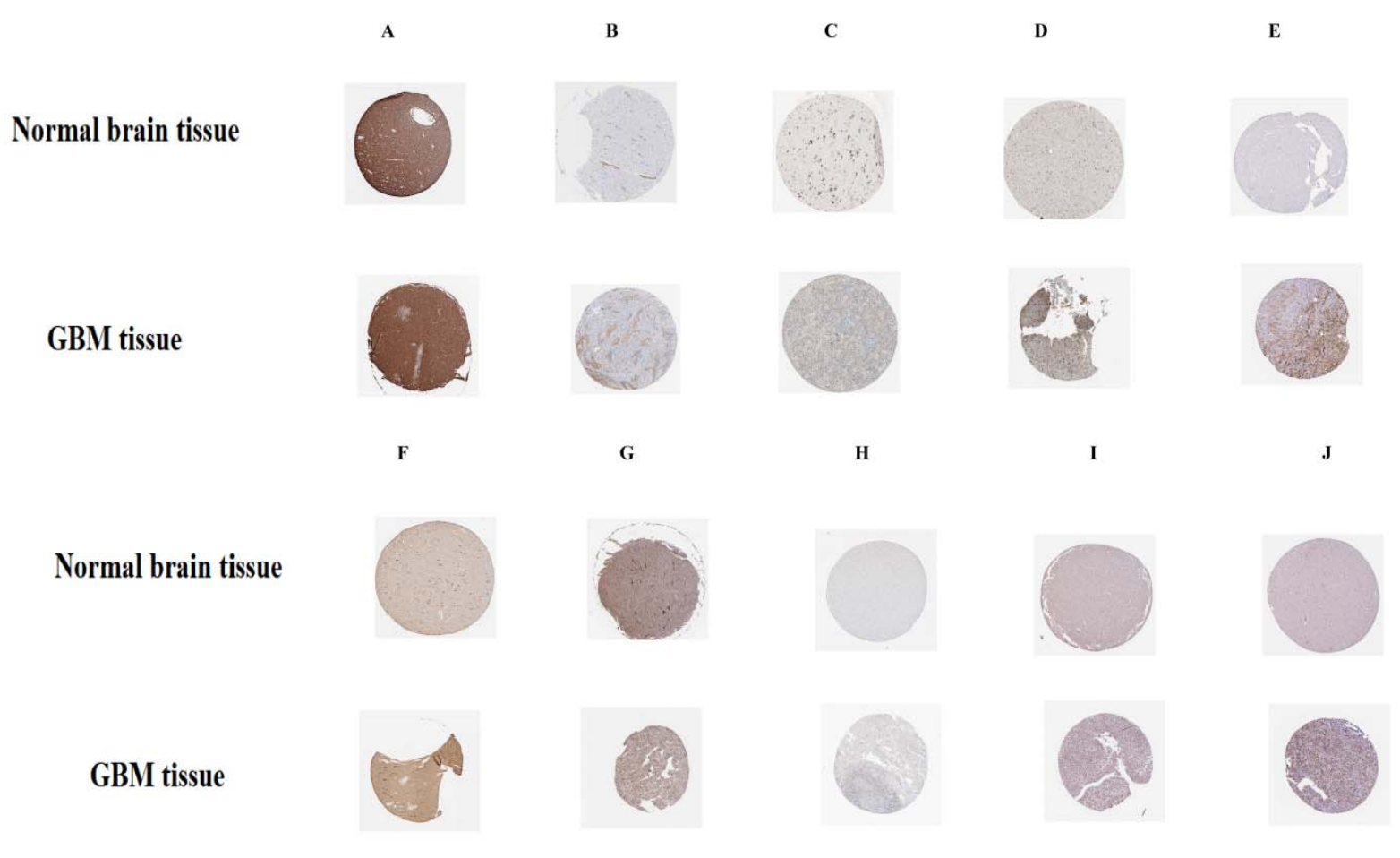

Fig. 20. Immunohisto chemical(IHC) analyses of hub genes were produced using the human protein atlas (HPA)

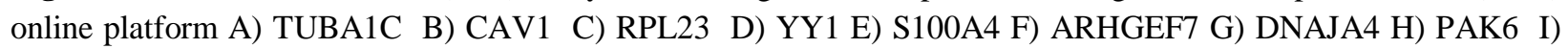
NELL1 J) ITPKA
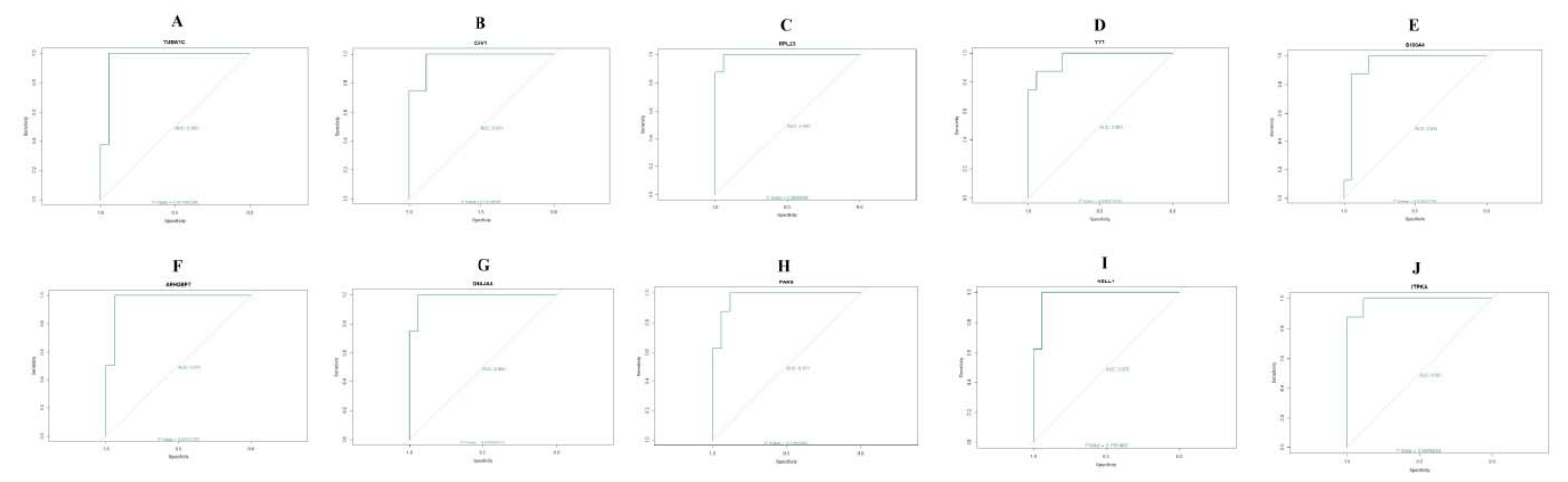

Fig. 21. ROC curve validated the sensitivity, specificity of hub genes as a predictive biomarker for GBM prognosis A) TUBA1C B) CAV1 C) RPL23 D) YY1 E) S100A4 F) ARHGEF7 G) DNAJA4 H) PAK6 I) NELL1 J) ITPKA 
medRxiv preprint doi: https://doi.org/10.1101/2020.12.21.20248616; this version posted December 23, 2020. The copyright holder for this preprint (which was not certified by peer review) is the author/funder, who has granted medRxiv a license to display the preprint in perpetuity. All rights reserved. No reuse allowed without permission.

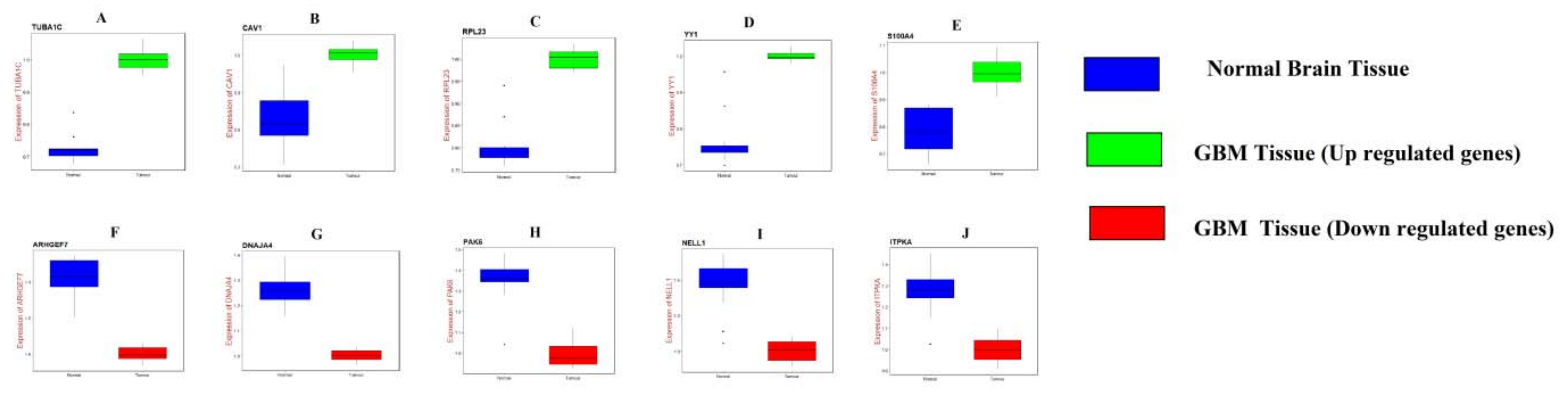

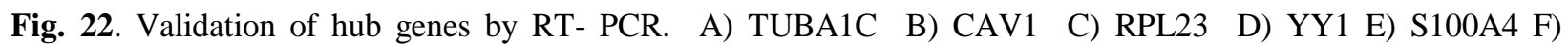
ARHGEF7 G) DNAJA4 H) PAK6 I) NELL1 J) ITPKA
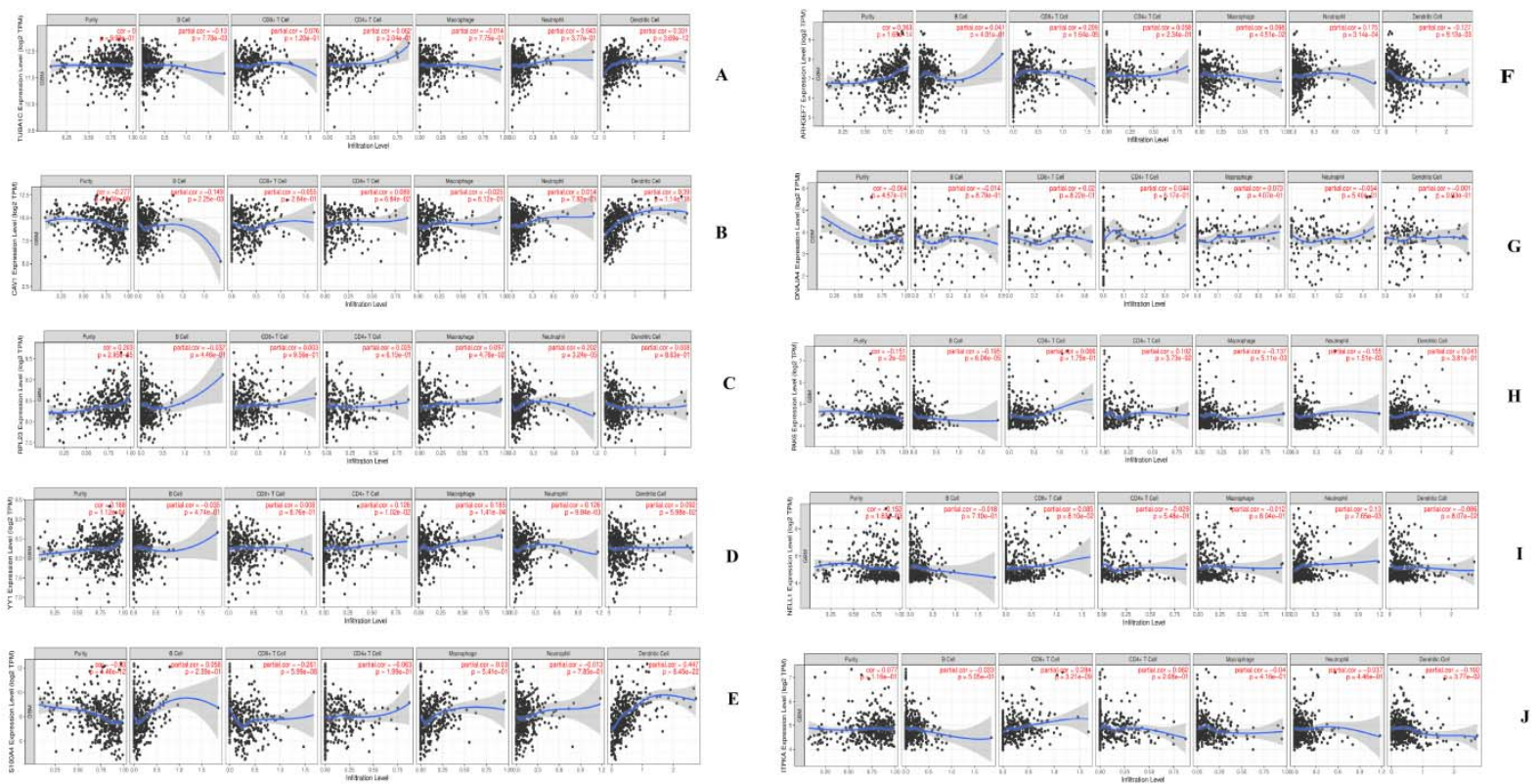

Fig. 23. Scatter plot for immune infiltration for hub genes. A) TUBA1C B) CAV1 C) RPL23 D) YY1 E) S100A4 F) ARHGEF7 G) DNAJA4 H) PAK6 I) NELL1 J) ITPKA 
medRxiv preprint doi: https://doi.org/10.1101/2020.12.21.20248616; this version posted December 23, 2020. The copyright holder for this preprint (which was not certified by peer review) is the author/funder, who has granted medRxiv a license to display the preprint in perpetuity.

All rights reserved. No reuse allowed without permission. 